\title{
New Crystalline Materials for Nonlinear Frequency Conversion, Electro-Optic Modulation, and Mid- Infrared Gain Media
}

\author{
J. J. Adams \\ Doctor of Philosophy in Engineering-Applied Science
}

\section{August 9, 2002}

Lawrence

Livermore

National

Laboratory

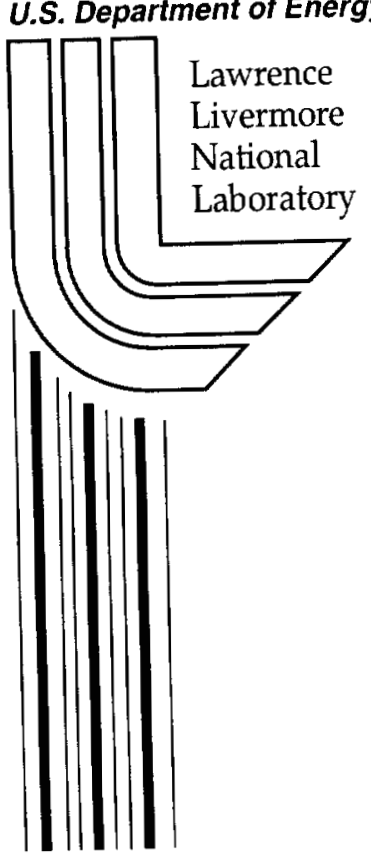




\section{DISCLAIMER}

This document was prepared as an account of work sponsored by an agency of the United States Government. Neither the United States Government nor the University of California nor any of their employees, makes any warranty, express or implied, or assumes any legal liability or responsibility for the accuracy, completeness, or usefulness of any information, apparatus, product, or process disclosed, or represents that its use would not infringe privately owned rights. Reference herein to any specific commercial product, process, or service by trade name, trademark, manufacturer, or otherwise, does not necessarily constitute or imply its endorsement, recommendation, or favoring by the United States Government or the University of California. The views and opinions of authors expressed herein do not necessarily state or reflect those of the United States Government or the University of California, and shall not be used for advertising or product endorsement purposes.

This work was performed under the auspices of the U.S. Department of Energy by the University of California, Lawrence Livermore National Laboratory under Contract No. W-7405-Eng-48.

This report has been reproduced directly from the best available copy.

Available electronically at http://www.doe.gov/bridge

Available for a processing fee to U.S. Department of Energy

and its contractors in paper from

U.S. Department of Energy

Office of Scientific and Technical Information

P.O. Box 62

Oak Ridge, TN 37831-0062

Telephone: (865) 576-8401

Facsimile: (865) 576-5728

E-mail: reports@adonis.osti.gov

Available for the sale to the public from

U.S. Department of Commerce

National Technical Information Service

5285 Port Royal Road

Springfield, VA 22161

Telephone: (800) 553-6847

Facsimile: (703) 605-6900

E-mail: orders@ntis.fedworld.gov

Online ordering: http://www.ntis.gov/ordering.htm

OR

Lawrence Livermore National Laboratory

Technical Information Department's Digital Library

http://www.Ilnl.gov/tid/Library.html 
New Crystalline Materials for Nonlinear Frequency Conversion, Electro-Optic Modulation, and Mid-Infrared Gain Media

BY

John Jonathan Adams

B.S. (University of California, Santa Barbara) 1990

M.S. (California State University, San Francisco) 1995

M.S. (University of California, Davis) 1998

DISSERTATION

Submitted in partial satisfaction of the requirements for the degree of

DOCTOR OF PHILOSOPHY

in

Engineering-Applied Science

in the

OFFICE OF GRADUATE STUDIES

of the

UNIVERSTTY OF CALIFORNIA

DAVIS

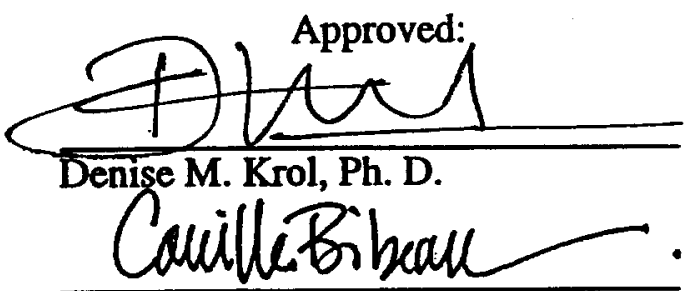

Camille Bibeau, Ph. D.

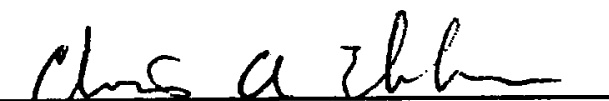

Chris A. Ebbers, Ph. D.

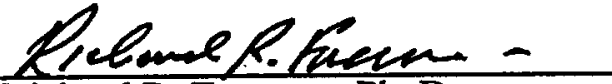

Richard R. Freeman, Ph. D.

Committee in Charge

2002 
Copyright (C) 2002

\section{By}

John J. Adams 
John J. Adams

September 2002

Engineering - Applied Science

\begin{abstract}
New Crystalline Materials for Nonlinear Frequency Conversion, Electro-Optic Modulation, and Mid-Infrared Gain Media
\end{abstract}

\begin{abstract}
$\underline{\text { Abstract }}$
New crystalline materials were investigated for applications in frequency conversion of near-infrared wavelengths and as gain media for tunable mid-infrared solid-state lasers. $\mathrm{GaCa} \mathrm{O}_{4} \mathrm{O}\left(\mathrm{BO}_{3}\right)_{3}(\mathrm{GdCOB}), \mathrm{YCa}_{4} \mathrm{O}\left(\mathrm{BO}_{3}\right)_{3}(\mathrm{YCOB}), \mathrm{LaCa}_{4} \mathrm{O}\left(\mathrm{BO}_{3}\right)_{3}(\mathrm{LaCOB})$, and $\mathrm{Gd}_{0.275} \mathrm{Y}_{0.725} \mathrm{Ca}_{4} \mathrm{O}\left(\mathrm{BO}_{3}\right)_{3}$ were characterized for frequency conversion of $1 \mu \mathrm{m}$ lasers. For type I doubling at $1064 \mathrm{~nm}, \mathrm{LaCOB}, \mathrm{GdCOB}$, and $\mathrm{YCOB}$ were found to have effective coupling coefficients $\left(d_{\text {eff }}\right)$ of $0.52 \pm 0.05,0.78 \pm 0.06$, and $1.12 \pm 0.07 \mathrm{pm} / \mathrm{V}$, respectively. LaCOB was measured to have angular and thermal sensitivities of $1224 \pm$ $184(\mathrm{~cm}-\mathrm{rad})^{-1}$ and $<0.10\left(\mathrm{~cm}^{\circ}{ }^{\circ} \mathrm{C}\right)^{-1}$, respectively. The effective coupling coefficient for type II non-critically phasematched (NCPM) doubling at $1064 \mathrm{~nm}$ in $\mathrm{Gd}_{0.275} \mathrm{Y}_{0.725} \mathrm{Ca}_{4} \mathrm{O}\left(\mathrm{BO}_{3}\right)_{3}$ was measured to be $0.37 \pm 0.04 \mathrm{pm} / \mathrm{V}$. We predict $\mathrm{LaCOB}$ to have a type I NCPM fundamental wavelength of $1042 \pm 1.5 \mathrm{~nm}$. Due to its low angular and thermal sensitivities for doubling near $1047 \mathrm{~nm}, \mathrm{LaCOB}$ has potential for frequency doubling of high-average power $\mathrm{Nd}: \mathrm{LiYF}_{4}$ and $\mathrm{Yb}: \mathrm{Sr}_{5}\left(\mathrm{PO}_{4}\right)_{3} \mathrm{~F}$ lasers. $\mathrm{LaCOB}, \mathrm{GdCOB}$, and YCOB were also investigated for optical parametric oscillator applications and we determined that they may have potential in a Ti:sapphire pumped oscillator.

The effective linear electro-optic coefficients $\left(r_{\text {eff }}\right)$ were measured along dielectric directions in $\mathrm{YCOB}$ and a maximum $r_{\mathrm{eff}}$ of $10.8 \mathrm{pm} / \mathrm{V}$ was found. For a crystal with a
\end{abstract}


5:1 aspect ratio, the corresponding half-wave voltage at $1064 \mathrm{~nm}$ would be $19.6 \mathrm{kV}$. Therefore a Pockels cell composed of two YCOB crystals with 5:1 aspect ratios would have a required half-wave voltage $<10 \mathrm{kV}$.

Moderate coupling coefficients $\left(3 \times \mathrm{KH}_{2} \mathrm{PO}_{4}\right)$, low thermal sensitivities, ease of growth to large sizes, non-hygroscopicity, and favorable polishing and coating characteristics make $\mathrm{LaCOB}, \mathrm{GdCOB}$, and $\mathrm{YCOB}$ attractive for frequency conversion of high-average power near-infrared lasers.

Absorption and emission cross-sections of $\sim 10^{-18} \mathrm{~cm}^{2}$ were measured for $\mathrm{Fe}^{2+}: \mathrm{ZnSe}$ in the $4 \mu \mathrm{m}$ region at temperatures below $220 \mathrm{~K}$. Luminescence lifetimes were found that ranged from $5-110 \mu$ selow $220 \mathrm{~K}$. Tunable lasing action was demonstrated for the first time in $\mathrm{Fe}^{2+}: \mathrm{ZnSe}$ with a tuning range from $3.98 \mu \mathrm{m}(20 \mathrm{~K})$ to $4.54 \mu \mathrm{m}(180 \mathrm{~K})$. The $\mathrm{Fe}^{2+}: \mathrm{ZnSe}$ laser had thresholds $\leq 50 \mu \mathrm{J}$ and slope efficiencies $\leq 10 \%$ with $0.6 \%$ output coupling. 


\section{Acknowledgments}

As I now find myself on the verge of completing this degree, I look back and would like to express my thanks to the numerous people who have inspired, supported, and assisted me throughout this long journey. First and foremost I would like to thank my mother who never once questioned my intentions or ever expressed doubts that I would complete my goal and who offered every possible method of support in tremendous amounts.

My development as a scientist and the quality of the research work for this degree is due in large part to the excellent set of advisors I have had in the Department of Applied Science (DAS) and in the Advanced Lasers and Components Group (AL\&C) at the Lawrence Livermore National Laboratory (LLNL). I would like to give Denise Krol my highest commendation for the superior job she did as my academic research advisor and committee chairperson. She is an example of a research advisor that all should follow. Steve Payne introduced me to my research topics and my work has benefited in immeasurable amounts by his oversight of the project. I also express thanks to Steve for encouraging me to publish my results and for the support to attend conferences and while finishing my dissertation. More directly involved with my day-to-day research activities were my laboratory research advisors Camille Bibeau and Chris Ebbers. I first would like to thank both Camille and Chris for taking me on as a graduate student. Camille taught me the basics of how to approach scientific research and how to express myself scientifically. I also would like to say thank you to Camille for the many discussions that we had concerning optical spectroscopy and laser materials. I would like to say thank 
you to Chris Ebbers for the opportunities he afforded me in the nonlinear materials portion of this work. I very much appreciate Chris' efforts to mold me into a wellprepared scientist.

I wish to say thank you to Kathleen Schaffers for growing several of the high quality laser and nonlinear crystals used in this study. I also thank Arnold Burger of Fisk University for several diffusion-doped samples. I thank Ralph Page for many technical and personal discussions and for his guidance on experimental techniques. I would also like to thank Eric Honea for many technical discussions and for being on my oral exam committee. I very much appreciate the willingness of Ray Beach and Luis Zapata to discuss laser physics with me and to answer a variety of technical questions.

A large portion of the work for this degree was in the laboratory setting which consisted of figuring out how to set up an experiment, how to make various pieces of equipment operate, and how to modify or repair equipment that I needed. I offer thanks to all those at LLNL or formerly at LLNL who have helped me with these and similar efforts. I would like to thank Larry Furu for first teaching me how to function in a laser laboratory and for his help in the first lasing of $\mathrm{Fe}^{2+}: \mathrm{ZnSe}$. Everett Utterback's masterful skills for figuring out how to make equipment work and for setting up automated dataacquisition systems were very much appreciated. I would like to express my deepest thanks to Everett for all his help. I also would like to express my thanks to Curt Cutter, Keith Kanz, John Lang, Scott Mitchell, Larry Smith, Joel Speth, John Tassano, and Gary Wilke who during the course of this work graciously lended their help in a variety of ways. I thank Peter Thelin, James Embree, and Ron Vallene for the excellent job of cutting and polishing all of the crystals that $I$ used in this study and I also thank Terry 
Duewer for the mass spectrometry analysis performed on the Fe-doped samples.

I would like to offer my deepest thanks to Mike Shaw of LLNL for all his help and for serving as one of my mentors. I would also like to say a special thank you to Rao Vemuri of DAS for his help in my entering this program and also for his assistance with my obtaining a student-employee position. I would like to thank Ann Orel of DAS for her very well taught courses and for being a strong advocate of my appointment to a student-employee position. I also appreciate the many discussions with Brian Kolner of DAS concerning the details of optics and the operation of laboratory equipment. I thank Rick Freeman, current chairman of DAS, for the guidance he has provided and for being a member of my committee and reading my dissertation. I would like to thank Jerry Fisher of San Francisco State University for the encouragement and support prior to and throughout my time in this program. I also thank the LLNL student-employee program for all their support.

I have greatly benefited from my interactions with my fellow DAS classmates. I would like to say a special thank you to Raj Patel for the many enjoyable lunches, technical discussions, study sessions, and discussions of life and politics. His positive outlook often gave a much needed boost to my motivation. I also would like to thank my office mates Andy Bayramian, Mike Nostrand, and Falgun Patel for the very enjoyable technical and social interactions.

From the inception of my post-secondary education, I have greatly benefited from the foresight of my grandfather J. J. Adams whom I thank for his wisdom and support. I also would like to thank Wells Fargo Bank for their role in my education. I have also highly benefited from the moral support given by my family and friends. To my brother Steve, 
my sister Cathy, and to my friends Van Glandon and Brian Dunning, I offer my sincerest thanks.

Performing the work necessary for this degree at LLNL was a unique experience that was a splice between a traditional university graduate program and the employment setting found in industry. Because of this, I had both academic and employment-related administrative needs. I would like to thank Archietta Johnson, Estelle Miller, Donna Clifford, and Jane Keene for helping keep my university administrative matters in order and Tammy Talovich, Kathy Allen, Kim Baynes, Delores Lambert, Frances Mendieta, Dustin Riggs, and Shelia Williams for taking care of my laboratory administrative needs. 


\section{Table of Contents}

Abstract.........................................................................................ii

Acknowledgments......................................................................iv

Chapter 1. Introduction .......................................................................................... 1

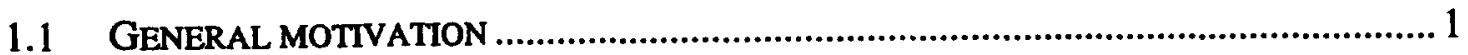

1.2 CONSIDERATIONS FOR NEW NONLINEAR MATERIALS ........................................ 2

1.2.1 COB family of nonlinear crystals............................................................... 3

1.3 CONSIDERATIONS FOR NEW TUNABLE MID-INFRARED LASER MATERIALS ............... 4

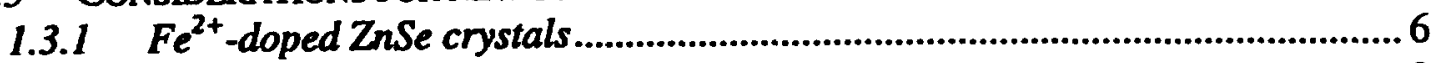

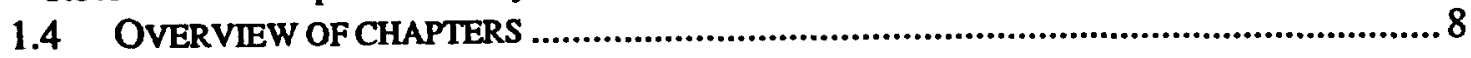

Chapter 2. Theoretical linear and nonlinear optics .............................................. 11

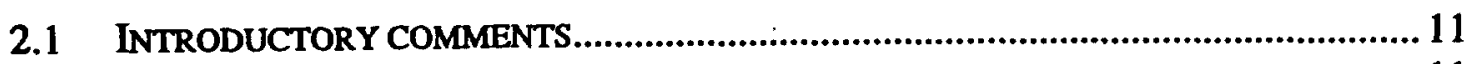

2.2 GENERAL LINEAR AND NONLINEAR OPTICAL PROPERTIES OF MATERIALS.............. 11

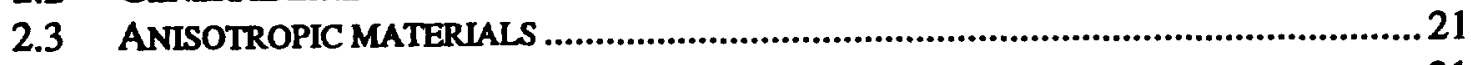

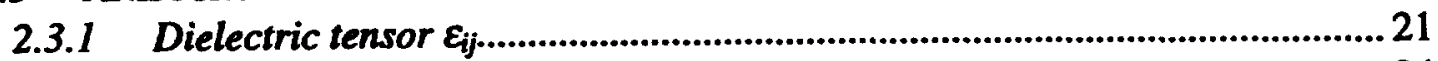

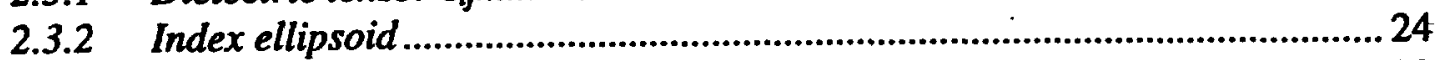

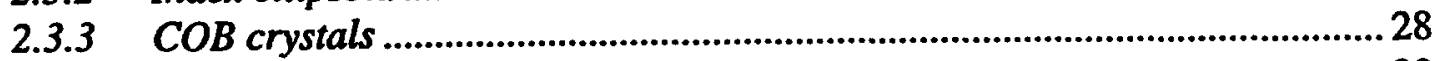

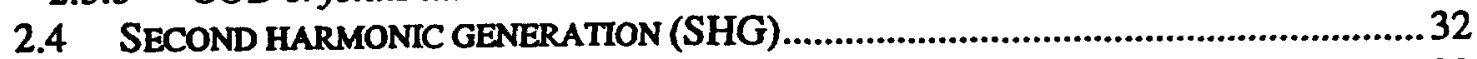

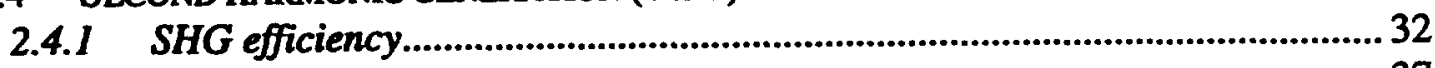

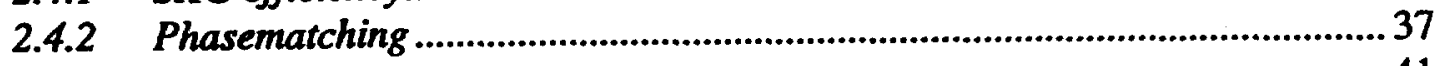

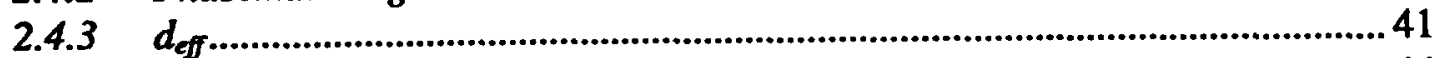

2.4.4 Angular, thermal, and spectral sensitivities................................................44

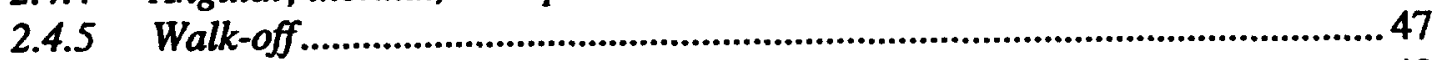

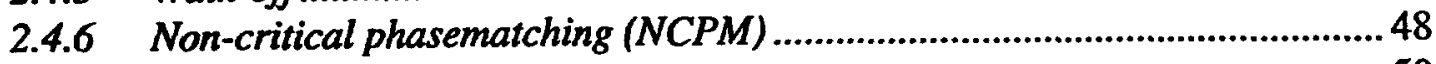

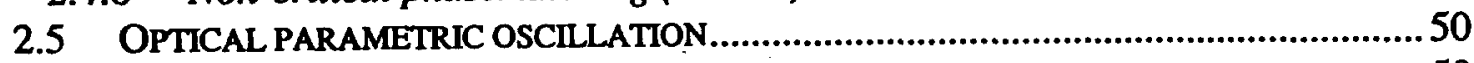

2.5.1 Coupled-amplitude equations .................................................................... 50

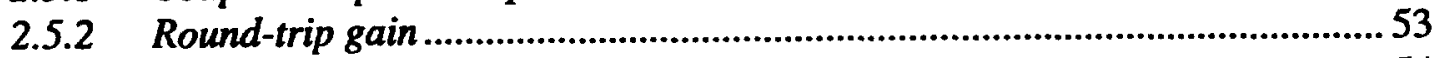

2.5.3 Oscillation threshold.................................................................................... 54

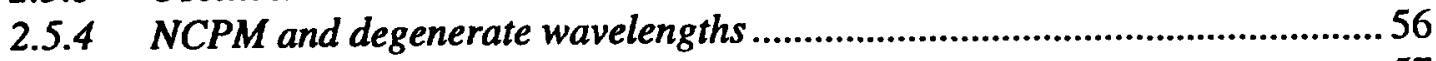

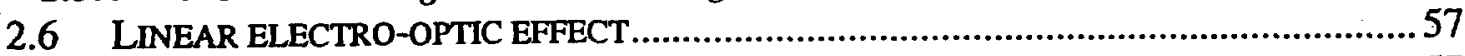

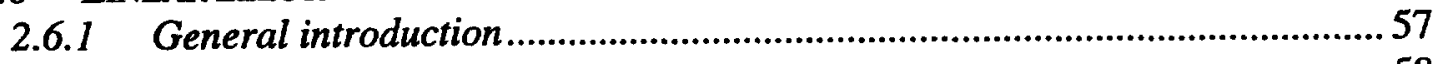

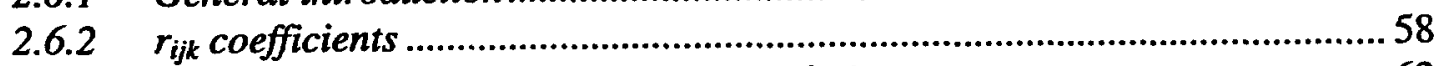

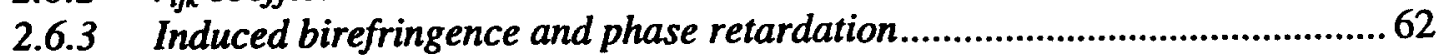

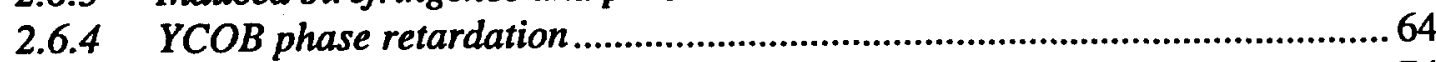

2.6.5 Transverse YCOB modulators................................................................... 74

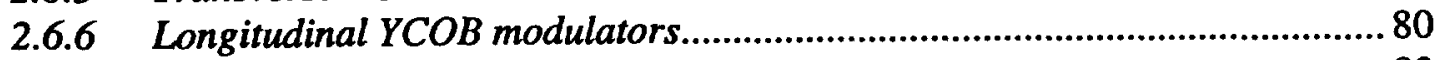

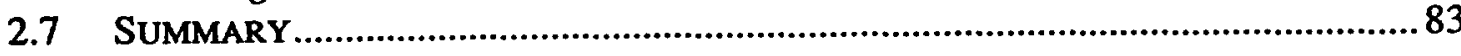


Chapter 3. Harmonic frequency conversion in $\mathrm{LaCa}_{4} \mathrm{O}\left(\mathrm{BO}_{3}\right)_{3}, \mathrm{GdCa}_{4} \mathrm{O}\left(\mathrm{BO}_{3}\right)_{3}$, $\mathrm{YCa}_{4} \mathrm{O}\left(\mathrm{BO}_{3}\right)_{3}$, and $\mathrm{Gd}_{0.275} \mathrm{Y}_{0.725} \mathrm{Ca}_{4} \mathrm{O}\left(\mathrm{BO}_{3}\right)_{3}$

3.1 INTRODUCTION

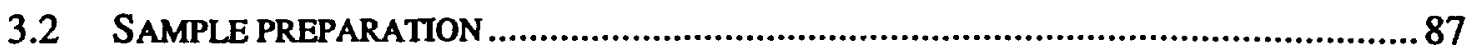

3.3 ANGLE-TUNED PHASEMATCHED SHG EXPERIMENTS ..........................................90

3.3.1 Effective coupling coefficients $\left(d_{\text {eff }}\right)$ and angular sensitivities $\left(\beta_{\theta}\right) \ldots \ldots \ldots \ldots . . . .90$

3.3.2 Calculation of the absolute nonlinear coupling coefficients $\left(d_{12}\right.$ and $\left.d_{32}\right)$....96

3.4 THERMAL SENSITTVITIES $\left(\beta_{T}\right)$ FOR SHG ............................................................97

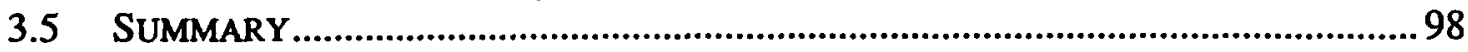

Chapter 4. Investigation of $\mathrm{YCa}_{4} \mathrm{O}\left(\mathrm{BO}_{3}\right)_{3}, \mathrm{GdCa} \mathrm{a}_{4} \mathrm{O}\left(\mathrm{BO}_{3}\right)_{3}$, and $\mathrm{LaCa}_{4} \mathrm{O}\left(\mathrm{BO}_{3}\right)_{3}$ for potential use in degenerate non-critically phasematched mid-infrared parametric oscillators .................................................................... 101

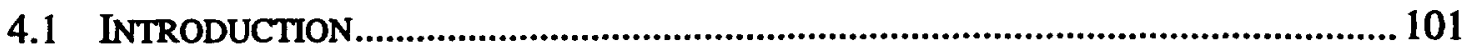

4.2 THEORETICAL ESTIMATES OF THE TYPE II NCPM OPO WAVEL ENGTHS, OPO THRESHOLD, AND SHG EFFICIENCY AND WAVELENGTH SENSITIVITY FOR

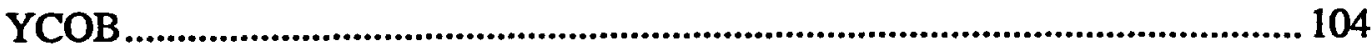

4.2.1 Non-critical OPO phasematching conditions ............................................... 104

4.2.2 Sellmeier equations...................................................................................... 105

4.2.3 Non-critically phasematched wavelengths.................................................... 105

4.2.4 Degenerate OPO threshold estimate.............................................................. 107

4.2.5 SHG efficiency estimate ................................................................................ 109

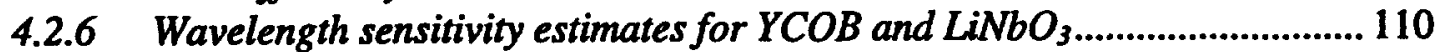

4.3 TYPE II NON-CRTICALLY PHASEMATCHED SHG EXPERIMENTS............................112

4.3.I General description of the experimental procedure ..................................... 112

4.3.2 Construction and characterization of a tunable $\mathrm{LiNbO}_{3} \mathrm{OPO}$.....................113

4.3.3 SHG experiments with the COB crystals........................................................ 118

4.4 DISCUSSION OF THE SHG RESPONSE ................................................................. 122

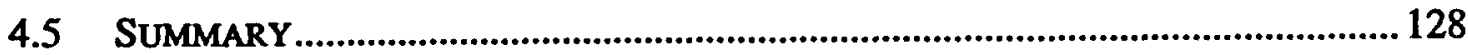

Chapter 5. Linear electro-optic properties of $\mathrm{YCa}_{4} \mathrm{O}\left(\mathrm{BO}_{3}\right)_{3}(\mathrm{YCOB}) \ldots \ldots \ldots . . . . . . . . . . .131$

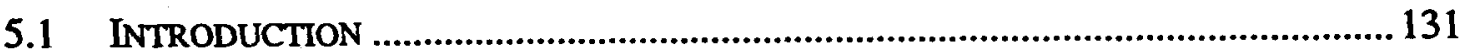

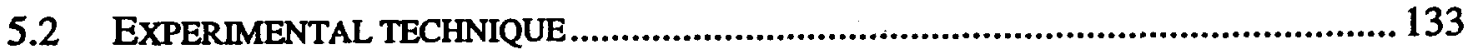

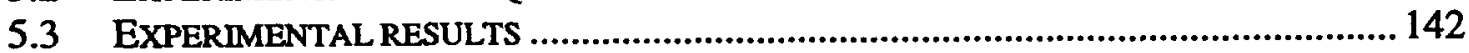

5.4 EFFECTIVE ELECTRO-OPTIC COEFFICIENTS AND HALF-WAVE VOLTAGES ............... 144

5.5 SUMMARY AND CONCLUSIONS ......................................................................... 147

Chapter 6. Optical spectroscopy of $\mathrm{Fe}^{2+}: \mathrm{ZnSe}$ crystals ...............................................150

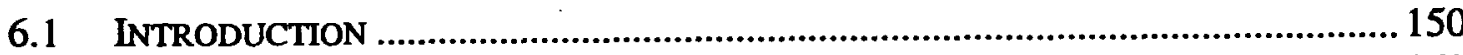

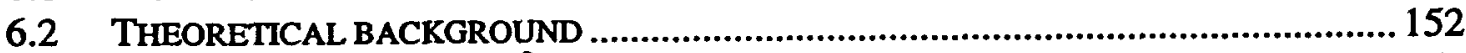

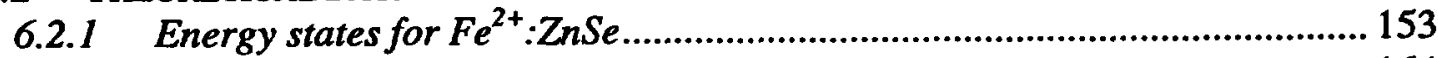

6.2.2 Configurational coordinate diagram ............................................................. 164 


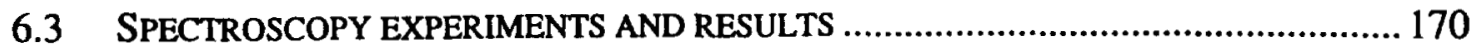

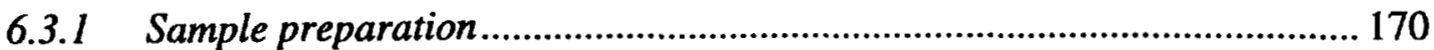

6.3.2 Temperature dependent absorption spectra............................................ 172

6.3.3 Temperature dependent emission spectra .................................................. 175

6.3.4 Temperature dependent luminescence lifetimes........................................ 178

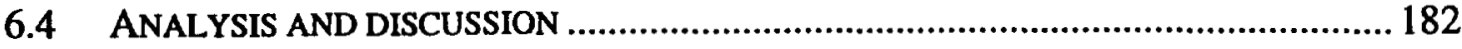

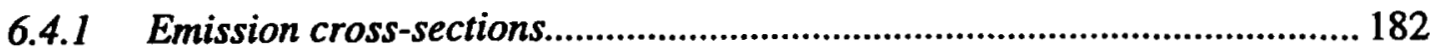

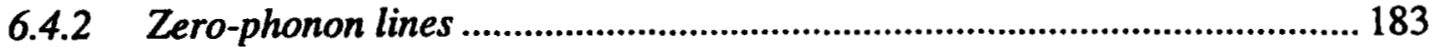

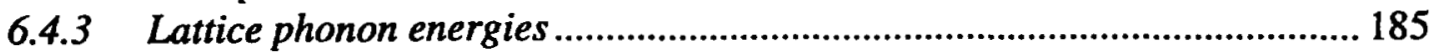

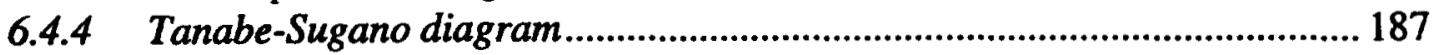

6.4.5 Huang-Rhys parameter and Condon offset ............................................. 189

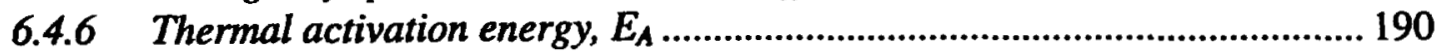

6.4.7 Configurational coordinate diagram ...................................................... 191

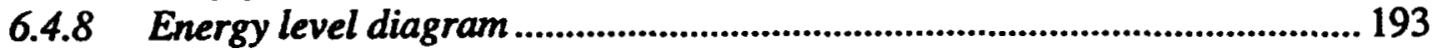

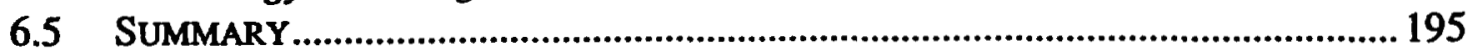

Chapter 7. $4.0-4.5 \mu \mathrm{m}$ laser performance of $\mathrm{Fe}^{2+}: \mathrm{ZnSe}$ below $180 \mathrm{~K} . . . \ldots \ldots \ldots \ldots \ldots \ldots . . . .0198$

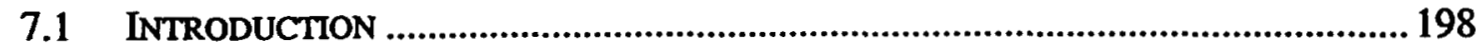

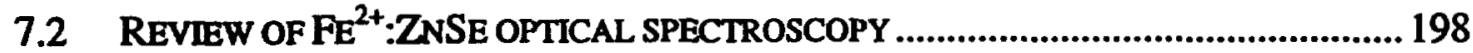

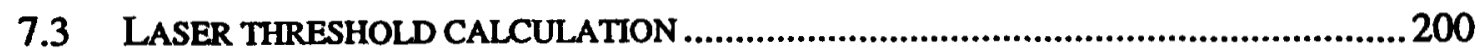

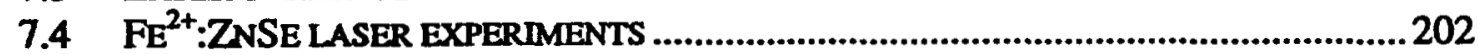

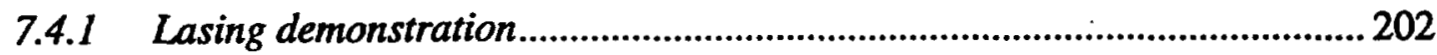

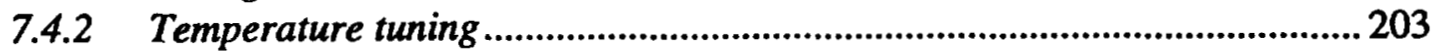

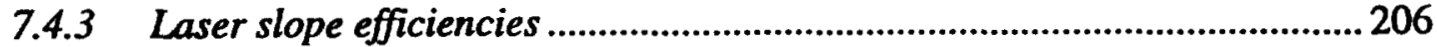

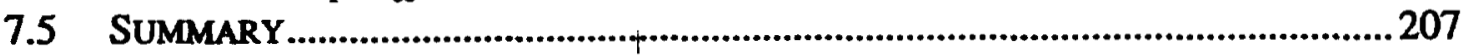

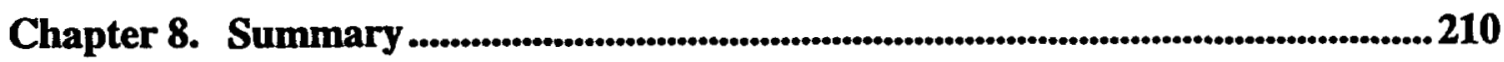




\section{Chapter 1}

\section{Introduction}

\subsection{General motivation}

The laser, invented in $1961[1,2]$, is such an extremely useful tool that it now pervades our life. For example, lasers can be found in the supermarket, at the dentist's office, in construction tools such as levels and theodolites, in compact disk players, and in the research and development laboratory. Lasers play a vital role in scientific research since they provide a bright, coherent, single color source of light [1-5]. As scientific research progresses new lasers are needed to meet the evolving needs. The Lawrence Livermore National Laboratory (LLNL) has programs that involve the development and deployment of many different kinds of new and novel laser systems for research applications [6-16].

One example of the work being conducted at LLNL is the development of nearinfrared (1 $\mu \mathrm{m})$ lasers for fusion energy research [6-11] and material processing. Novel near-infrared laser systems such as these utilize nonlinear crystals for frequency conversion (since many applications require the second or third harmonic of the fundamental wavelength) and for polarization Bontrol (Pockels cells) [17-20]. As stateof-the-art laser systems are engineered, unique requirements are placed on the nonlinear crystals used for frequency conversion that are not easily satisfied by commercially available nonlinear crystals. Therefore as frequency conversion requirements extend to unprecedented regimes, new nonlinear crystals need to be identified and developed.

Another example of new lasers being developed at LLNL is tunable mid-infrared solid-state lasers for use in remote sensing and laser radar [12-16]. Tunable mid-infrared 
(>2 $\mu \mathrm{m}$ ) laser materials are scarce which makes the development of these systems difficult. In order to satisfy these demands for tunable mid-infrared solid-state lasers, new mid-infrared laser crystals need to be discovered.

\subsection{Considerations for new nonlinear materials}

In general, nonlinear optical crystals need to satisfy a basic set of materials' requirements in order to be attractive for frequency conversion applications. In all cases, insensitivity to moisture is highly desirable. A material that is insensitive to moisture can be used in ambient conditions without any special handling or packaging requirements. The commercially available nonlinear crystals that are commonly used for frequency conversion and polarization control in $1 \mu \mathrm{m}$ lasers are $\mathrm{KH}_{2} \mathrm{PO}_{4}(\mathrm{KDP}), \mathrm{KD}_{2} \mathrm{PO}_{4}(\mathrm{KD} * \mathrm{P})$, $\mathrm{LiB}_{3} \mathrm{O}_{5}$ (LBO), $\mathrm{BaB}_{2} \mathrm{O}_{4}(\mathrm{BBO})$, and $\mathrm{KTiOPO}_{4}$ (KTP). Among these nonlinear crystals, $\mathrm{KD}_{2} \mathrm{PO}_{4}(\mathrm{KD} * \mathrm{P})$ and to a lesser extent $\mathrm{LiB}_{3} \mathrm{O}_{5}(\mathrm{LBO})$ and $\mathrm{BaB}_{2} \mathrm{O}_{4}(\mathrm{BBO})$ exhibit sensitivity to moisture. Further desired properties for a nonlinear crystal would be a large nonlinear coupling coefficient, low thermal and angular sensitivities, and a high damage threshold.

As solid-state lasers are built that have larger apertures $\left(\sim 10 \mathrm{~cm}^{2}\right)$ and produce higher average powers $(-10 \mathrm{~kW})[6,9,11]$, there is increased need for nonlinear optical crystals which can be grown to large sizes. Of the commercially available crystals, only KDP and KD*P can be grown to large sizes $\left(15 \mathrm{~cm}^{3}\right)$ and each of these has certain materials' "issues" that make them less than ideal for large aperture, high-average power applications. The first part of this study will focus on identifying attractive new nonlinear crystals for frequency conversion of near-infrared lasers that have the potential to be grown to large sizes. 


\subsubsection{COB family of nonlinear crystals}

The $\mathrm{COB}$ family of crystals $\left(\mathrm{GdCa}_{4} \mathrm{O}\left(\mathrm{BO}_{3}\right)_{3}(\mathrm{Gd}, \mathrm{COB}), \mathrm{YCa}_{4} \mathrm{O}\left(\mathrm{BO}_{3}\right)_{3}(\mathrm{YCOB})\right.$, $\mathrm{Gd}_{0.275} \mathrm{Y}_{0.725} \mathrm{Ca} 4 \mathrm{O}\left(\mathrm{BO}_{3}\right)_{3}(\mathrm{Gd}, \mathrm{YCOB})$, etc. $)$ has recently been introduced [21-23] as a promising new class of nonlinear crystals, capable of frequency conversion of near infrared wavelengths. All of the crystals in the $\mathrm{COB}$ family are monoclinic and belong to the m point symmetry class. The crystal structure for GdCOB is shown in Fig. 1.1. The COB crystals possess the basic properties that make them desirable as optical materials. They are insensitive to moisture, have surface damage thresholds of at least $30 \mathrm{~J} / \mathrm{cm}^{2}$ [24], have high transparency from $-200-2200 \mathrm{~nm}$, and are easy to polish and optically coat. They can also be easily grown to large sizes. Typical boules grown using the Czochralski pulling method [25] are approximately $5 \mathrm{~cm}$ in diameter by $15 \mathrm{~cm}$ in length. These crystals have been previously investigated at LLNL as a potential replacement for LBO and KTP in intracavity frequency doubling applications. They have also found applications in tripling of Nd:YAG lasers [22,23].

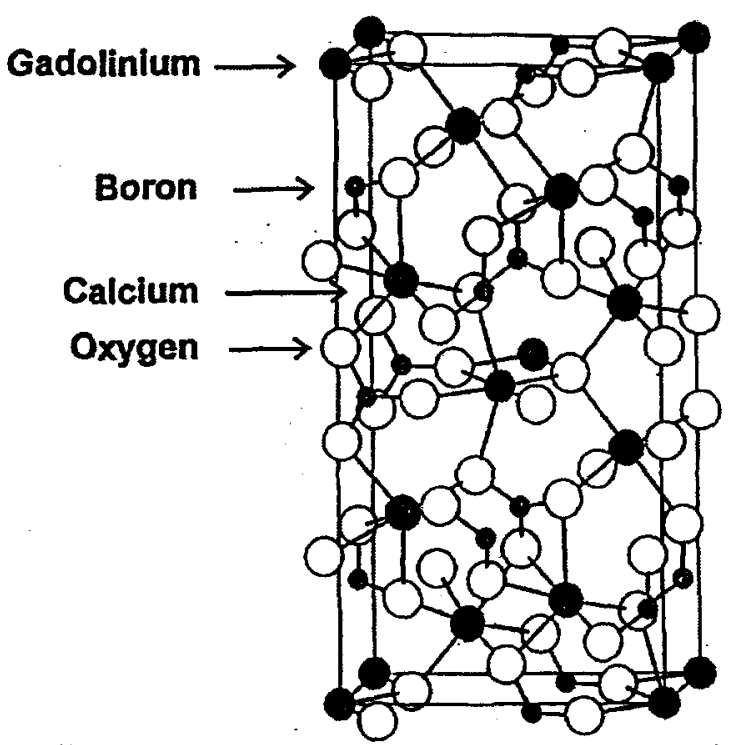

Figure 1.1. Crystal structure for GdCOB [23]. 
In the first part of this dissertation (Chapters $2-5$ ), we will experimentally characterize the nonlinear optical properties of $\mathrm{GdCOB}, \mathrm{YCOB}, \mathrm{Gd}, \mathrm{YCOB}$, and the newest member to this family $\mathrm{LaCa}_{4} \mathrm{O}\left(\mathrm{BO}_{3}\right)_{3}(\mathrm{LaCOB})$ relevant to frequency conversion of $1 \mu \mathrm{m}$ lasers. The values reported by other groups for the coupling coefficients for type I doubling at $1064 \mathrm{~nm}$ in GdCOB and $\mathrm{YCOB}$ are inconsistent (i.e. a variance of $~ 30 \%$ ) [26-28]. Therefore, we will first conduct experiments to accurately characterize type I doubling at $1064 \mathrm{~nm}$ in the COB crystals. From the doubling experiments, we will find that the COB crystals possess at least one large nonlinear coupling coefficient $\left(\mathrm{d}_{32}\right)$. One application based on this large nonlinear coupling coefficient that we will further investigate is their use in a non-critically phasematched optical parametric oscillator. A large nonlinear coupling coefficient may also indicate the existence of a correspondingly large linear electro-optic coefficient [19]. Therefore, we will also conduct experiments to determine if indeed a correspondingly large linear electro-optic coefficient $\left(\mathrm{I}_{23}\right)$ does exist by measuring the effective electro-optic coefficients for YCOB.

\subsection{Considerations for new tunable mid-infrared laser materials}

The wavelength(s) needed for a specific remote sensing application depends for instance, on the molecular species that is to be detected [12]. Many organic molecular species have absorption bands that lie at wavelengths $\geqq 1.4 \mu \mathrm{m}$ which requires that a midinfrared laser be used in the remote sensing system [12]. Typically, the laser used in a remote sensing system also needs to offer tunability in its emission wavelength so that detection techniques such as resonance scattering and differential absorption can be used [12]. The specific remote sensing applications that we are trying to address require a 
laser that provides widely tunable emission in the $3-5 \mu \mathrm{m}$ region. In the $3-5 \mu \mathrm{m}$ region, wide tunability is approximately $500 \mathrm{~nm}$ [13].

Optical parametric oscillators [5] and direct solid-state lasers are two types of lasers that are commonly used for generating tunable laser emission in the mid-infrared. By direct solid-state lasers we mean lasers that produce the desired emission from an electronic transition in a material rather than a non-linear interaction. We would like to use direct solid-state lasers for these applications rather than an optical parametric oscillator (OPO) since direct solid-state lasers are simpler, more compact optical systems to work with.

There are very few direct solid-state laser materials that have emission wavelengths greater than approximately $2 \mu \mathrm{m}$ and that also offer significant tunability. As shown in Fig. 1.2, there are at present no commercially available widely tunable solid-state laser materials that emit past approximately $2.8 \mu \mathrm{m}$. The most recent member of the tunable materials shown in Fig. 1.2 is $\mathrm{Cr}^{2+}: \mathrm{ZnSe} . \mathrm{Cr}^{2+}: \mathrm{ZnSe}$ was discovered to be a widely

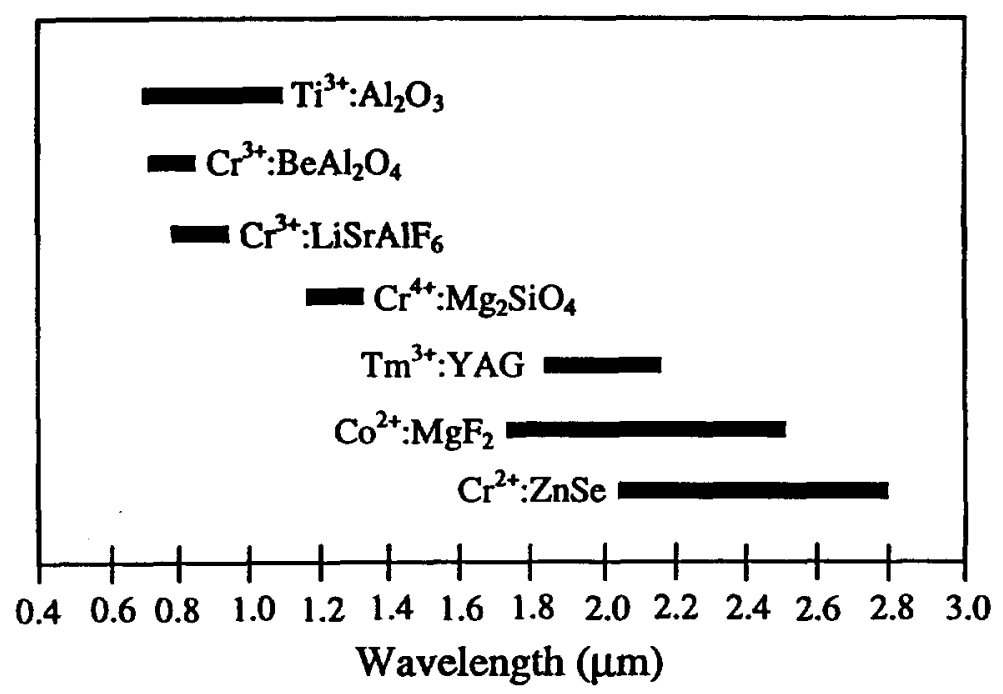

Figure 1.2. Wavelength range of commercially available widely tunable solid-state lasers [5]. 
tunable laser material in the $2-3 \mu \mathrm{m}$ range by Deloach et al. at LLNL in $1996[29,30]$.

Though the absorption and luminescence properties of various transition metal-doped zinc chalcogenides (i.e. $\mathrm{Cr}^{2+}: \mathrm{ZnSe}, \mathrm{Fe}^{2+}: \mathrm{ZnSe}, \mathrm{Ni}^{2+}: \mathrm{ZnSe}, \mathrm{Co}^{2+}: \mathrm{ZnSe}, \mathrm{Cr}^{2+}: \mathrm{ZnS}$, $\mathrm{Fe}^{2+}: \mathrm{ZnS}, \mathrm{Cr}^{2+}: \mathrm{ZnTe}, \mathrm{Co}^{2+}: \mathrm{ZnTe}$, etc.) have been much discussed in the literature over the past 35 years, no one had investigated their potential as laser materials until the work by DeLoach et al. in 1996 [29]. DeLoach et al. [29] examined crystals of $\mathrm{ZnSe}, \mathrm{ZnS}$, and $\mathrm{ZnTe}$ individually doped with $\mathrm{Cr}^{2+}, \mathrm{Co}^{2+}, \mathrm{Ni}^{2+}$, and $\mathrm{Fe}^{2+}$. They reported that $\mathrm{Fe}^{2+}: \mathrm{ZnSe}$ had a broad absorption band centered at $\sim 2900 \mathrm{~nm}$ at room temperature but they did not observe any luminescence. In 1967, Haanstra reported [31] that at a sample temperature of approximately $18 \mathrm{~K}, \mathrm{Fe}^{2+}: \mathrm{ZnSe}$ had an absorption band centered at $3200 \mathrm{~nm}$ and an emission band centered at $4000 \mathrm{~nm}$ with a width of approximately $600 \mathrm{~nm}$. Therefore, $\mathrm{Fe}^{2+}: \mathrm{ZnSe}$ may have potential as a widely tunable $3-5 \mu \mathrm{m}$, albeit cryogenic, laser material.

\subsection{1 $\mathrm{Fe}^{2+}$-doped ZnSe crystals}

The zinc-chalcogenides (i.e. $\mathrm{ZnSe}, \mathrm{ZnS}, \mathrm{ZnTe}$, etc.) have materials' properties that make them good hosts for mid-infrared lasers. One very favorable property is that they transmit visible through far infrared $(>15 \mu \mathrm{m})$ wavelengths. Another favorable property is that they allow substitutional incorporation of transition metal ions (i.e. they can be doped with transition metals). Transition metal-doped samples can be produced by the Bridgman growth method [25] or by diffusion doping [32]. Both of these methods are relatively inexpensive (especially diffusion doping) and can be used to quickly produce doped-crystals ( 0.1 at. \%) with high optical quality. 
$\mathrm{ZnSe}$ has cubic symmetry and is commonly referred to as the sphalerite structure. The crystal structure for $\mathrm{ZnSe}$ is shown in Fig. 1.3. Notice that each $\mathrm{Zn}^{2+}$ ion has four

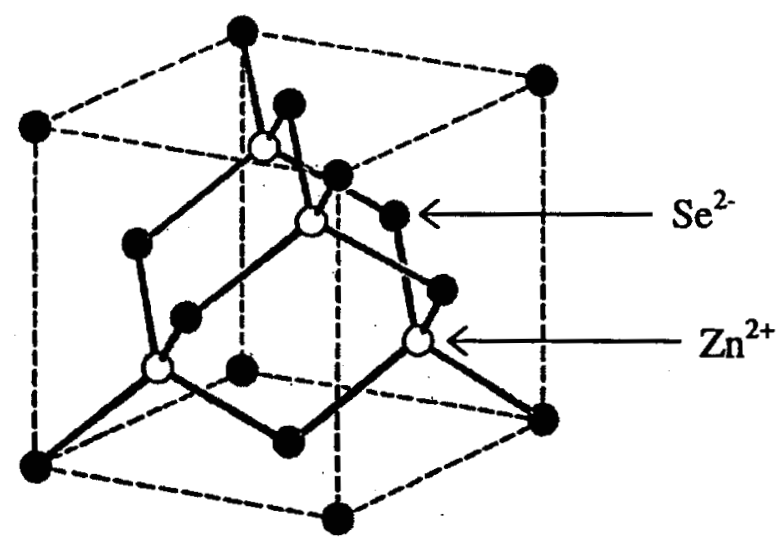

Figure 1.3. Crystal structure for $\mathrm{ZnSe}$ [33].

tetrahedrally coordinated $\mathrm{Se}^{2-}$ ions as nearest neighbors. When transition metal impurities are incorporated into the ZnSe lattice, previous spectroscopic studies [34,35] have shown that they are primarily doubly ionized and preferentially substitute into $\mathrm{Zn}^{2+}$ sites. Therefore for the case of $\mathrm{Fe}^{2+}$ impurities in $\mathrm{ZnSe}$ (i.e. $\mathrm{Fe}^{2+}: \mathrm{ZnSe}$ ), each $\mathrm{Fe}^{2+}$ ion is in a tetrahedral coordination with four $\mathrm{Se}^{2-}$ ions. The crystal field due to the $\mathrm{Se}^{2-}$ ions splits the ${ }^{5} \mathrm{D}$ state of the $\mathrm{Fe}^{2+}$ ion into a ${ }^{5} \mathrm{E}$ and a ${ }^{5} \mathrm{~T}_{2}$ state [36] as shown in Fig. 1.4, with a

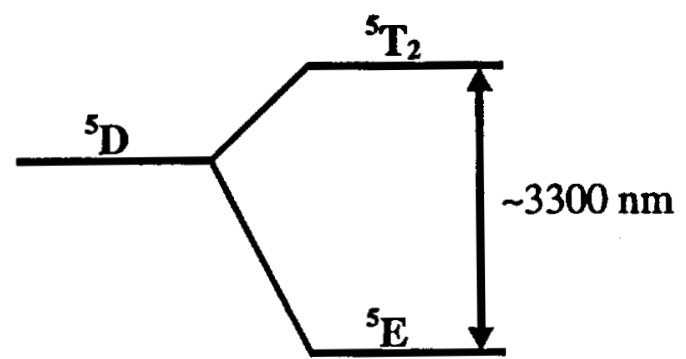
Free-ion
Tetrahedral
state
Crystal Field
splitting

Figure 1.4. Splitting of the ${ }^{5} \mathrm{D}$ state for $\mathrm{Fe}^{2+}: \mathrm{ZnSe}$. 
splitting of approximately $3300 \mathrm{~nm}$ [31]. Dipole transitions [36] are allowed between the ${ }^{5} \mathrm{~T}_{2}$ and ${ }^{5} \mathrm{E}$ states and so it is this transition in $\mathrm{Fe}^{2+}: \mathrm{ZnSe}$ that we would like to exploit for widely tunable $3-5 \mu \mathrm{m}$ lasing action. The second part of this dissertation (Chapters 6 and 7) presents the results from the evaluation of $\mathrm{Fe}^{2+}: \mathrm{ZnSe}$ as a widely tunable $3-5 \mu \mathrm{m}$ laser material.

\subsection{Overview of chapters}

The remaining chapters in this dissertation present and discuss the necessary concepts, experimental procedures, experimental results, and conclusions from the study of the nonlinear optical properties of the $\mathrm{COB}$ family of crystals and from the spectroscopic and laser studies of $\mathrm{Fe}^{2+}: \mathrm{ZnSe}$. Chapter 2 discusses the theoretical nonlinear optics concepts that are necessary to understand and analyze the experiments conducted with the COB crystals in Chapters 3, 4, and 5. Chapter 3 discusses the experimental characterization of second harmonic generation in the $\mathrm{COB}$ crystals. In Chapter 4, we experimentally investigate the use of the COB crystals in a degenerate, non-critically phasematched optical parametric oscillator. Chapter 5 describes the experimental measurement of the effective linear electro-optic coefficients for YCOB. Chapter 6 presents and discusses the experimentally measured spectroscopic properties of $\mathrm{Fe}^{2+}: \mathrm{ZnSe}$ that are relevant to predicting its potential to reach laser threshold. In Chapter 7, we describe the first laser demonstration of $\mathrm{Fe}^{2+}: \mathrm{ZnSe}$ and its performance characteristics. Chapter 8 summarizes the major results and conclusions determined in this work. 


\section{References for Chapter 1}

[1] T. H. Maiman, Nature 187, 493 (1960)

[2] T. H. Maiman, Phys. Rev. Letters 4, 564 (1960)

[3] A. E. Siegman, Lasers (University Science Books, Sausalito, 1986)

[4] R. C. Powell, Physics of Solid-State Laser Materials (Springer-Verlag, New York, 1998)

[5] W. Koechner, Solid-State Laser Engineering, $4^{\text {th }}$ Edition (Springer-Verlag, Berlin, 1996)

[6] W. J. Hogan, E. I. Moses, B. E. Warner, M. S. Sorem, and J. M. Soures, Nuc. Fus. 41, 567 (2001)

[7] D. Eimerl, IEEE J. Quantum Electron. 23, 575 (1987)

[8] C. D. Orth, S. A. Payne, and W. F. Krupke, Nuc. Fus. 36, 75 (1996)

[9] C. D. Marshall, et al., Laser Physics 8, 741 (1998)

[10] C. D. Marshall, et al., Optical Society of America Trends in Optics and Photonics Series 19, 318 (1998)

[11] R. L. McCrory, et al., Nuc. Fus. 41, 1413 (2001)

[12] R. M. Measures, Laser Remote Sensing: Fundamentals and Applications, (John Wiley and Sons, New York, 1984)

[13] E. Zanzottera, Crit. Rev. Anal. Chem. 21, 279 (1990)

[14] T. P. Grayson, et al., Proc. IEEE NAECON 2, 678 (1996)

[15] S. A. Payne, et al., Proc. SPIE 2833, 153 (1996)

[16] J. C. Diettrich, I. T. McKinnie, and D. M. Warrington, in OSA Trends in Optics and Photonics (TOPS) Vol. 56, Conference on Lasers and Electro-Optics (CLEO 2001),Technical Digest, Postconference Edition, 502 (2001)

[17] J. A. Armstrong, N. Bloembergen, J. Ducuing, and P. S. Pershan, Phys. Rev. 127, 1918 (1962)

[18] F. Zemike and J. E. Midwinter, Applied Nonlinear Optics, (John Wiley and Sons, Inc., New York, 1973)

[19] A. Yariv and P. Yeh, Optical Waves in Crystals - Propagation and Control of Laser Radiation, (John Wiley and Sons, Inc., New York, 1984)

[20] R. W. Boyd, Nonlinear Optics, (Academic Press, San Diego, California, 1992)

[21] R. Norrestam, M. Nygren, and J.-O. Bovin, Chem. Mater. 4, 737 (1992)

[22] M. Iwai, T. Kobayashi, H. Furuya, Y. Mori, and T. Sasaki, Jpn. J. Appl. Phys. 36, L276 (1997)

[23] G. Aka, A. Kahn-Harari, F. Mougel, D. Vivien, F. Salin, P. Coquelin, P. Colin, D. Pelenc, and J. Damelet, J. Opt. Soc. Am. B 14, 2238 (1997)

[24] M. Runkel, private communication, LLNL (2001)

[25] H. E. Ruda, Widegap II-VI Compounds for Opto-electronic Applications, (Chapman and Hall, London, 1992)

[26] F. Mougel, G. Aka, F. Salin, D. Pelenc, B. Ferrand, A. Kahn-Harari, and D. Vivien, Optical Society of America Trends in Optics and Photonics Series 26, 709 (1999)

[27] C. Chen, Z. Shao, J. Jiang, J. Wei, J. Lin, J. Wang, N. Ye, J. Lu, B. Wu, M. Jiang, M. Yoshimura, Y. Mori, and T. Sasaki, J. Opt. Soc. Am. B 17, 566 (2000) 
[28] G. Aka, A. Kahn-Harari, D. Vivien, J.-M. Benitez, F. Salin, and J. Godard, Eur. J. Solid State Inorg. Chem. 33, 727 (1996)

[29] L. D. DeLoach, R. H. Page, G. D. Wilke, S. A. Payne, and W. F. Krupke, IEEE J. Quan. Elec. 32, 885 (1996)

[30] R. H. Page, K. I. Schaffers, L. D. DeLoach, G. D. Wilke, F. D. Patel, J. B. Tassano, Jr., S. A. Payne, W. F. Krupke, K.-T. Chen, and A. Burger, IEEE J. Quan. Elec. 33, 609 (1997)

[31] J. H. Haanstra, II-VI Semiconducting Compounds 1967 International Conference, D. G. Thomas (ed.), 207 (W. A. Benjamin, Inc., New York, 1967)

[32] C. I. Rablau, J.-O. Ndap, X. Ma, A. Burger, and N. C. Giles, J. Electron. Mater. 28, 678 (1999)

[33] C. Kittel, Introduction to Solid State Physics, (John Wiley and Sons, Inc., New York, 1996)

[34] J. M. Baranowski, J. W. Allen, and G. L. Pearson, Phys. Rev. 160, 627 (1967)

[35] G. A. Slack, F. S. Ham, and R. M. Chrenko, Phys. Rev. 152, 376 (1966)

[36] F. A. Cotton, Chemical Applications of Group Theory, $3^{\text {rd }}$ Edition (Wiley, New York, 1971) 


\section{Chapter 2}

\section{Theoretical linear and nonlinear optics}

\subsection{Introductory comments}

$\mathrm{GdCa}_{4} \mathrm{O}\left(\mathrm{BO}_{3}\right)_{3} \quad(\mathrm{GdCOB}), \quad \mathrm{YCa} a_{4} \mathrm{O}\left(\mathrm{BO}_{3}\right)_{3} \quad(\mathrm{YCOB})$, and $\mathrm{Gd}_{0.25}, \mathrm{Y}_{0.75} \mathrm{Ca}_{4} \mathrm{O}\left(\mathrm{BO}_{3}\right)_{3}$ (Gd,YCOB) are three isostructural members of the COB family of crystals, previously presented in the literature [1-4], whose nonlinear optical properties will be characterized in the following three chapters. A new member to the COB family of crystals, $\mathrm{LaCa}_{4} \mathrm{O}\left(\mathrm{BO}_{3}\right)_{3}(\mathrm{LaCOB})$, is introduced and also characterized. The concepts and equations developed here will be used to analyze the experimental data measured for the COB crystals in the next three chapters.

This chapter will develop the basic mathematical description for several linear and nonlinear effects that arise from a material's first-order $\left(\chi^{(1)}\right)$ and second-order $\left(\chi^{(2)}\right)$ optical susceptibility. In section 2.2 , we begin by discussing linear and nonlinear optical properties of materials in general. The linear optics for biaxial crystals and the conventions for describing the $\mathrm{COB}$ crystal structure then follows in section 2.3 . Second harmonic generation is discussed for biaxial crystals in section 2.4 , optical parametric oscillation in section 2.5, and the linear electro-optic effect in section 2.6. Section 2.7 summarizes the chapter. All quantities and equations will be in the c.g.s. system of units unless otherwise noted.

\subsection{General linear and nonlinear optical properties of materials}

The electromagnetic response of a material to an applied optical field can be described by how the dipole moment per unit volume, or the polarization of the material 
$(\overrightarrow{\mathrm{P}})$, depends on the magnitude of the applied optical field $(\vec{E})$. In the general case, $\overrightarrow{\mathrm{P}}$ and $\overrightarrow{\mathrm{E}}$ are vectors and $\chi$ is a tensor, however for the purposes of this immediate discussion we will restrict ourselves to one dimension and treat $\mathrm{P}, \mathrm{E}$, and $\chi$ as scalars. The nonlinear optical response of a material can be described by expressing the polarization (P) as a power series [5] in the applied optical field (E) as

$$
\mathrm{P}=\chi^{(1)} \mathrm{E}+\chi^{(2)} \mathrm{E}^{2}+\chi^{(3)} \mathrm{E}^{3}+\cdots
$$

The quantities $\chi^{(1)}, \chi^{(2)}$, and $\chi^{(3)}$, are known as the linear, second-order, and third-order optical susceptibilities, respectively. The first term on the right-hand side of Eqn. (2.1) is the linear polarization, the second term is the second-order nonlinear polarization (which is quadratically dependent on the applied electric field), and the third term is the thirdorder nonlinear polarization. We will focus for the moment only on the second-order term that involves the coefficient $\chi^{(2)}$.

Let's consider the second-order coupling between two scalar fields with frequencies $\omega_{1}$ and $\omega_{2}$ and determine what frequencies are produced by their interaction. We choose to write the two interacting fields as infinite plane-waves given by

and

$$
E_{1}=\left(E_{1}\left(\omega_{1}\right) e^{-i \omega_{1} t}+\text { c.c. }\right)
$$

$$
E_{2}=\left(E_{2}\left(\omega_{2}\right) e_{2}^{-i \omega_{2} t}+c . c .\right)
$$

where the coefficients $E_{1}\left(\omega_{1}\right)$ and $E_{2}\left(\omega_{2}\right)$ are complex numbers and c.c. means complex conjugate. We write the second-order polarization as a sum over all combinations of positive and negative frequencies (i.e. $\pm \omega_{1}$ and $\pm \omega_{2}$ ) as 


$$
P^{(2)}=\sum_{n} P^{(2)}\left(\omega_{n}\right) e^{-i \omega_{n} t}
$$

where the amplitudes $\mathrm{P}^{(2)}\left(\omega_{n}\right)$ are complex numbers. Substituting Eqns. (2.2), (2.3), and (2.4) into the second-order term in Eqn. (2.1) and equating terms yields the following relations between the complex amplitudes of the different frequency components of the second-order polarization and the amplitudes of the electric fields.

$P^{(2)}\left(2 \omega_{1}\right)=\chi^{(2)} E_{1}\left(\omega_{1}\right)^{2} \quad$ (Second Harmonic Generation (SHG))

$P^{(2)}\left(2 \omega_{2}\right)=\chi^{(2)} E_{2}\left(\omega_{2}\right)^{2} \quad(S H G)$

$P^{(2)}\left(\omega_{1}+\omega_{2}\right)=2 \chi^{(2)} E_{1}\left(\omega_{1}\right) E_{2}\left(\omega_{2}\right) \quad$ (Sum Frequency Generation (SFG))

$P^{(2)}\left(\omega_{1}-\omega_{2}\right)=2 \chi^{(2)} E_{1}\left(\omega_{1}\right) E_{2}^{*}\left(\omega_{2}\right) \quad$ (Difference Frequency Generation (DFG))

$P^{(2)}(0)=2 \chi^{(2)}\left(E_{1}\left(\omega_{1}\right) E_{1}^{*}\left(\omega_{1}\right)+E_{2}\left(\omega_{2}\right) E_{2}\left(\omega_{2}\right)^{*}\right) \quad$ (Optical Rectification)

We have assumed here that $\chi^{(2)}$ is independent of frequency. We find that the interaction of two frequencies results in the generation of five different frequencies (including one at zero frequency). The interactions are identified according to standard nonlinear optics nomenclature [5]. The factor of two in the expressions for the sum and difference frequencies arise because there are two distinct fields interacting as opposed to a single field interacting with itself; therefore twice the contribution.

An important fundamental property of $\chi^{(2)}$ can be deduced from the SHG term in Eqn. (2.5) $\left(\mathrm{P}^{(2)}\left(2 \omega_{1}\right)=\chi^{(2)} \mathrm{E}_{1}\left(\omega_{1}\right)^{2}\right)$. In a material that possesses inversion symmetry, under the inversion operation $\mathrm{E} \rightarrow-\mathrm{E}$ and $\mathrm{P} \rightarrow-\mathrm{P}$. The SHG term then gives

$$
-\mathrm{P}^{(2)}\left(2 \omega_{1}\right)=\chi^{(2)}\left(-\mathrm{E}_{1}\left(\omega_{1}\right)\right)\left(-\mathrm{E}_{1}\left(\omega_{1}\right)\right)=\chi^{(2)} \mathrm{E}_{1}\left(\omega_{1}\right)^{2}
$$

The second equality implies that $-\mathrm{P}^{(2)}\left(2 \omega_{1}\right)=\mathrm{P}^{(2)}\left(2 \omega_{1}\right)$ which can only hold if $\chi^{(2)}$ equals 
zero. Therefore we have shown a fundamental property for materials that possess inversion symmetry: Materials that possess inversion symmetry will have $\chi^{(2)}=0$. As an example, gases and liquids are centrosymmetric materials which have $\chi^{(2)}=0$.

We also see from Eqn. (2.5) that a polarization with zero frequency is produced or in other words a DC electric field is established in the material. This is known as optical rectification. The symmetry properties of $\chi^{(2)}$ (as will be discussed shortly) imply a reciprocal effect that a DC field applied to the material can be used to change the phase of the input wave [6]. This can be described as SFG where one field at $\omega_{1}=\omega$ and another field at $\omega_{2}=0$ (DC) are applied to the material which produces a third field at $\omega_{3}$ $=\omega+0=\omega$ that has a phase change relative to $\omega_{1}$. This is known as the electro-optic effect [5]. We would like to therefore note that the electro-optic effect is a second-order nonlinear process that is generated through a material's $\chi^{(2)}$.

All of the processes described in Eqn. (2.5) arise through a materials $\chi^{(2)}$. SHG, SFG, DFG, and optical rectification (electro-optic effect) can be viewed as $\chi^{(2)}$ processes each with distinct values for the interacting frequencies. The experimental work in the next three chapters studies SHG, SFG, and the electro-optic effect and so we will limit the following discussion to nonlinear properties which arise from $\chi^{(2)}$. We will further limit our discussion to second-order interactions between just three fields, $E\left(\omega_{1}\right), E\left(\omega_{2}\right)$, and $\mathrm{E}\left(\omega_{3}\right)$. We will now proceed with developing a more useful mathematical description of sum frequency generation, second harmonic generation, and the electro-optic effect involving a maximum of three applied fields.

Eqn. (2.6) and Eqn. (2.7) are the general forms we will use for the electric fields and 


$$
\begin{aligned}
& E_{i}(\vec{r}, t)=\sum_{n} E_{i}\left(\omega_{n}\right) e^{i\left(\vec{k}_{n} \cdot \vec{r}-\omega_{n} t\right)} \\
& P_{i}(\vec{r}, t)=\sum_{n} P_{i}\left(\omega_{n}\right) e^{i\left(\vec{k}_{n} \cdot \vec{r}-\omega_{n} t\right)}
\end{aligned}
$$

polarizations. The index $\mathrm{i}$ specifies the cartesian components, the index $\mathrm{n}$ is a sum over all positive and negative frequencies, the amplitudes $E_{i}\left(\omega_{n}\right)$ and $P_{i}\left(\omega_{n}\right)$ are complex numbers, $\vec{k}_{n}$ is the wavevector at frequency $\omega_{n}$, and $\vec{r}$ is the position vector. For the 3 fields that we will consider, $n$ runs over $\pm 1, \pm 2$, and \pm 3 . We have not shown any explicit spatial dependence for $E_{i}\left(\omega_{n}\right)$ and $P_{i}\left(\omega_{n}\right)$ because we will assume that they are spatially slowly-varying field amplitudes. Also, $\omega_{\mathrm{n}}$ should not be regarded as the argument of a function but rather a parameter index. The fields in Eqns. (2.6) and (2.7) are physically observable and hence must be real quantities and so the complex amplitudes must satisfy

$$
E_{i}\left(-\omega_{n}\right)=E_{i}\left(\omega_{n}\right) * \text { and } P_{i}\left(-\omega_{n}\right)=P_{i}\left(\omega_{n}\right) *
$$

We would now like to generalize Eqn. (2.5) to three-dimensions and write it in a much more compact form. In one-dimension, $\mathrm{E}, \mathrm{P}$, and $\chi$ were scalars. In threedimensions, $\mathrm{E}$ and $\mathrm{P}$ become vectors and have three orthogonal components each. $\chi^{(2)}$ becomes a third rank tensor $\left(\chi_{\mathrm{ijk}}{ }^{(2)}\left(\omega_{n}+\omega_{\mathrm{m}} ; \omega_{\mathrm{n}}, \omega_{\mathrm{n}}\right)\right)$ and multiplies two vectors to form a third. The three-dimensional form we will assert for Eqn. (2.5) will be given without a derivation. It is a standard result that may be found in many books [5-7]. We define the components of the second-order susceptibility tensor $\left(\chi_{\mathrm{ijk}}^{(2)}\left(\omega_{n}+\omega_{\mathrm{m}} ; \omega_{n}, \omega_{\mathrm{m}}\right)\right)$ as the constants of proportionality relating the amplitude of the nonlinear polarization to the product of the field amplitudes by 


$$
P_{i}\left(\omega_{n}+\omega_{m}\right)=\sum_{j k} \sum_{(n m)} \chi_{i j k}^{(2)}\left(\omega_{n}+\omega_{m} ; \omega_{n}, \omega_{m}\right) E_{j}\left(\omega_{n}\right) E_{k}\left(\omega_{m}\right)
$$

The indices $i, j$, and $k$ each refer to the three cartesian components of the fields (i.e. $i$ can refer to $x, y$, or $z$ ). The notation $(\mathrm{nm})$ indicates that, in performing the summation over $\mathrm{n}$ and $m$, the sum $\omega_{n}+\omega_{m}$ is to be held fixed (i.e. $\omega_{3}=\omega_{1}+\omega_{2}$ ) while the frequencies are permuted (in this case $\omega_{1}$ and $\omega_{2}$ are permuted). We have used the notation $\chi^{(2)}=$ $\chi_{i j k}^{(2)}\left(\omega_{n}+\omega_{m} ; \omega_{n}, \omega_{m}\right)$ to indicate that in general $\chi_{i j k}^{(2)}$ depends on all three frequencies and the order in which they appear.

We will write out the polarization components for the interactions of three fields $\left(E\left(\omega_{1}\right), E\left(\omega_{2}\right)\right.$, and $\left.E\left(\omega_{3}\right)\right)$ for the cases of SFG and SHG to illustrate how to use Eqn. (2.9). First let's consider SFG. Let the applied frequencies be $\omega_{1}$ and $\omega_{2}$ and the sum frequency be $\omega_{3}$, such that $\omega_{3}=\omega_{1}+\omega_{2}$. By doing the summation over $\omega_{n}$ and $\omega_{\mathrm{m}}$ in Eqn. (2.9) we obtain

$$
P_{i}\left(\omega_{3}\right)=\sum_{j k}\left[\chi_{i j k}^{(2)}\left(\omega_{3} ; \omega_{1}, \omega_{2}\right) E_{j}\left(\omega_{1}\right) E_{k}\left(\omega_{2}\right)+\chi_{i j k}^{(2)}\left(\omega_{3} ; \omega_{2}, \omega_{1}\right) E_{j}\left(\omega_{2}\right) E_{k}\left(\omega_{1}\right)\right]
$$

The nonlinear susceptibility has a property known as intrinsic permutation symmetry. Intrinsic permutation symmetry implies that reversing the order of $\omega_{1}$ and $\omega_{2}$ in $\chi_{\mathrm{ijk}}{ }^{(2)}\left(\omega_{3}\right.$; $\left.\omega_{1}, \omega_{2}\right)$ does not change its numerical value as long as the indices $\mathrm{j}$ and $\mathrm{k}$ are permuted as well. Physically this can be seen from Eqn. (2.9). The order the applied fields were written in Eqn. (2.9) was arbitrary and could just as well have been written with $\mathbf{n}$ and $\mathrm{m}$ and $\mathrm{j}$ and $\mathrm{k}$ interchanged without affecting the value of the term. In other words, the numerical values of the un-permuted $\left(\chi_{\mathrm{ijk}}{ }^{(2)}\left(\omega_{3} ; \omega_{1}, \omega_{2}\right)\right)$ and permuted $\left(\chi_{\mathrm{ikj}}{ }^{(2)}\left(\omega_{3} ; \omega_{2}, \omega_{1}\right)\right)$ 
susceptibilities are equal. Mathematically, intrinsic permutation symmetry can be expressed by

$$
\chi_{\mathrm{ijk}}^{(2)}\left(\omega_{3} ; \omega_{1}, \omega_{2}\right)=\chi_{\mathrm{ikj}}^{(2)}\left(\omega_{3} ; \omega_{2}, \omega_{1}\right)
$$

Application of intrinsic permutation symmetry reduces Eqn. (2.10) to

$$
P_{i}\left(\omega_{3}\right)=2 \sum_{j k} \chi_{i j k}^{(2)}\left(\omega_{3} ; \omega_{1}, \omega_{2}\right) E_{j}\left(\omega_{1}\right) E_{k}\left(\omega_{2}\right)
$$

where still $\omega_{3}=\omega_{1}+\omega_{2}$.

The interaction of all three frequencies is accounted for in Eqn. (2.9), that is $E\left(\omega_{3}\right)$ generated by $E\left(\omega_{1}\right)$ and $E\left(\omega_{2}\right), E\left(\omega_{1}\right)$ generated by $E\left(\omega_{3}\right)$ and $E\left(\omega_{2}\right)$, etc. It will be instructive now and useful later to write out how the different frequency components are related for SFG according to Eqn. (2.9). We are only interested in the frequency components in Eqn. (2.9) that are involved in SFG, i.e. $P\left(\omega_{1}\right), P\left(\omega_{2}\right)$, and $P\left(\omega_{3}\right)$, so permuting the frequencies, performing the summation, identifying the sum frequency terms, and applying Eqn. (2.8) gives

$$
\begin{aligned}
& P_{i}\left(\omega_{1}\right)=2 \sum_{j k} \chi_{i j k}^{(2)}\left(\omega_{1} ; \omega_{3},-\omega_{2}\right) E_{j}\left(\omega_{3}\right) E_{k}\left(\omega_{2}\right)^{*} \\
& P_{i}\left(\omega_{2}\right)=2 \sum_{j k} \chi_{i j k}^{(2)}\left(\omega_{2} ; \omega_{3},-\omega_{1}\right) E_{j}\left(\omega_{3}\right) E_{k}\left(\omega_{1}\right)^{*} \\
& P_{i}\left(\omega_{3}\right)=2 \sum_{j k} \chi_{i j k}^{(2)}\left(\omega_{3} ; \omega_{1}, \omega_{2}\right) E_{j}\left(\omega_{1}\right) E_{k}\left(\omega_{2}\right)
\end{aligned}
$$

where we only show the terms with the positive frequencies however there are three more terms for the negative frequencies. The equations in Eqn. (2.13) were found by rewriting Eqn. (2.12) using $\omega_{1}=\omega_{3}-\omega_{2}$, etc. Also note in Eqn. (2.13), the index i specifies either 
$\mathrm{x}, \mathrm{y}$, or $\mathrm{z}$ for each frequency component. These equations will be useful later in this chapter for writing down the coupled amplitude equations for SFG.

Next we consider SHG. This is a special case of SFG where $\omega_{2}=\omega_{1}$ and $\omega_{3}=2 \omega_{1}$. The input frequency is taken as $\omega_{1}$ and the generated harmonic as $\omega_{3}=2 \omega_{1}$. Again performing the summation over $\omega_{n}$ and $\omega_{m}$ in Eqn. (2.9) we obtain

$$
P_{i}\left(\omega_{3}\right)=\sum_{j k} \chi_{i j k}^{(2)}\left(\omega_{3} ; \omega_{1}, \omega_{1}\right) E_{j}\left(\omega_{1}\right) E_{k}\left(\omega_{1}\right)
$$

where $\omega_{3}=\omega_{1}+\omega_{1}$. Once again the interaction of both frequencies is accounted for in Eqn. (2.9), that is $E\left(\omega_{3}\right)$ generated by $E\left(\omega_{1}\right)$, and $E\left(\omega_{1}\right)$ generated by $E\left(\omega_{1}\right)$ and $E\left(\omega_{3}\right)$. It will be useful later to have written out how the different frequency components are related for SHG according to Eqn. (2.9). We are only interested in the frequency components in Eqn. (2.9) that are involved in SHG, i.e. $P\left(\omega_{1}\right)$ and $P\left(\omega_{3}\right)$, so permuting the frequencies, performing the summation, identifying the harmonic terms, and applying Eqn. (2.8) gives

$$
\begin{aligned}
& P_{i}\left(\omega_{1}\right)=2 \sum_{j k} \chi_{i j k}^{(2)}\left(\omega_{1} ; \omega_{3},-\omega_{1}\right) E_{j}\left(\omega_{3}\right) E_{k}\left(\omega_{1}\right)^{*} \\
& P_{i}\left(\omega_{3}\right)=\sum_{j k} \chi_{i j k}^{(2)}\left(\omega_{3} ; \omega_{1}, \omega_{1}\right) E_{j}\left(\omega_{1}\right) E_{k}\left(\omega_{1}\right)
\end{aligned}
$$

where we only show the terms with the positive frequencies however there are two more terms for the negative frequencies. These equations will be useful later in this chapter for writing down the coupled amplitude equations for SHG.

A lossless material gives rise to two other useful symmetries obeyed by $\chi_{\mathrm{ijk}}{ }^{(2)}$. The 
first is that all of the components of $\chi_{\mathrm{ijk}}^{(2)}$ are real. The second symmetry obeyed by $\chi_{\mathrm{ijk}}{ }^{(2)}$ for a lossless material is full permutation symmetry. Full permutation symmetry means that all of the frequency components of $\chi_{\mathrm{ijk}}^{(2)}$ can be freely interchanged as long as the corresponding cartesian indices are interchanged simultaneously. Neither of these two symmetry properties of $\chi_{i j k}^{(2)}$ will be proven here, however the proof that $\chi_{\mathrm{ijk}}{ }^{(2)}$ is real follows from considering a classical anharmonic oscillator and can be found in Ref. [6] and the proof of full permutation symmetry follows from considering the electromagnetic energy density in a lossless medium and can be found in Ref. [5].

When nonlinear optical interactions are occurring for frequencies that are far from any resonant frequency of the material, the nonlinear susceptibility is essentially independent of frequency [5]. This is known as Kleinman symmetry. Kleinman symmetry implies that the indices of $\chi_{\mathrm{ijk}}{ }^{(2)}$ can be permuted without permuting the frequencies since $\chi_{\mathrm{ijk}}^{(2)}$ is independent of frequency. Eqn. (2.16) shows one of the equalities satisfied by $\chi_{\mathrm{ijk}}^{(2)}$ when Kleinman symmetry is valid.

$$
\chi_{\mathrm{ijk}}^{(2)}\left(\omega_{3} ; \omega_{1}, \omega_{2}\right)=\chi_{\mathrm{jki}}^{(2)}\left(\omega_{3} ; \omega_{1}, \omega_{2}\right)
$$

When Kleinman symmetry is valid it is common practice [5-7] to introduce the $d_{i j k}$ tensor used by experimentalists. The $\mathrm{d}_{\mathrm{ijk}}$ tensor is defined by

$$
\mathrm{d}_{\mathrm{ijk}} \equiv \frac{1}{2} \chi_{\mathrm{ijk}}^{(2)}
$$

where there are no frequency indexes on $\chi_{\mathrm{ijk}}^{(2)}$ or $\mathrm{d}_{\mathrm{ijk}}$ because both are assumed to be independent of frequency (Kleinman symmetry). The components of the $\mathrm{d}_{\mathrm{ijk}}$ tensor are 
referred to as the nonlinear coupling coefficients. Using Eqn. (2.17), Eqns. (2.12) and (2.14) become

$$
P_{i}\left(\omega_{3}=\omega_{1}+\omega_{2}\right)=4 \sum_{j k} d_{i j k} E_{j}\left(\omega_{1}\right) E_{k}\left(\omega_{2}\right) \quad(S F G)
$$

and

$$
P_{i}\left(\omega_{3}=2 \omega_{1}\right)=2 \sum_{j k} d_{i j k} E_{j}\left(\omega_{1}\right) E_{k}\left(\omega_{1}\right) \quad(S H G)
$$

Intrinsic permutation symmetry implies that $d_{i j k}$ is symmetric in its last two indices. This allows the notation to be simplified by contracting the last two indices on $\mathrm{d}_{\mathrm{ijk}}$ (i.e. $\left.\mathrm{d}_{\mathrm{il}}\right)$ according to

$$
\begin{array}{llllccc}
\text { jk: } & 11 & 22 & 33 & 23,32 & 31,13 & 12,21 \\
\text { l: } & 1 & 2 & 3 & 4 & 5 & 6
\end{array}
$$

The tensor $\mathrm{d}_{\mathrm{il}}$ can then be written in terms of a $3 \times 6$ matrix as

$$
d_{i 1}=\left[\begin{array}{llllll}
d_{11} & d_{12} & d_{13} & d_{14} & d_{15} & d_{16} \\
d_{21} & d_{22} & d_{23} & d_{24} & d_{25} & d_{26} \\
d_{31} & d_{32} & d_{33} & d_{34} & d_{35} & d_{36}
\end{array}\right]
$$

This notation has explicitly reduced the number of independent components of $d_{\mathrm{ijk}}$ to 18 . If we now impose the Kleinman symmetry condition, then $d_{12}=d_{26}, d_{14}=d_{25}$, etc. and Eqn. (2.21) becomes

$$
d_{i 1}=\left[\begin{array}{llllll}
d_{11} & d_{12} & d_{13} & d_{14} & d_{15} & d_{16} \\
d_{16} & d_{22} & d_{23} & d_{24} & d_{14} & d_{12} \\
d_{15} & d_{24} & d_{33} & d_{23} & d_{13} & d_{14}
\end{array}\right]
$$

Here the number of independent components has dropped to 10 . When Kleinman 
symmetry is valid, we can write Eqn. (2.18) (SFG) in terms of the $d_{i l}$ coefficients in a matrix equation as

$$
\left[\begin{array}{l}
P_{x}\left(\omega_{3}\right) \\
P_{y}\left(\omega_{3}\right) \\
P_{z}\left(\omega_{3}\right)
\end{array}\right]=4\left[\begin{array}{llllll}
d_{11} & d_{12} & d_{13} & d_{14} & d_{15} & d_{16} \\
d_{21} & d_{22} & d_{23} & d_{24} & d_{25} & d_{26} \\
d_{31} & d_{32} & d_{33} & d_{34} & d_{35} & d_{36}
\end{array}\right]\left[\begin{array}{c}
E_{x}\left(\omega_{1}\right) E_{x}\left(\omega_{2}\right) \\
E_{y}\left(\omega_{1}\right) E_{y}\left(\omega_{2}\right) \\
E_{z}\left(\omega_{1}\right) E_{z}\left(\omega_{2}\right) \\
E_{y}\left(\omega_{1}\right) E_{z}\left(\omega_{2}\right)+E_{z}\left(\omega_{1}\right) E_{y}\left(\omega_{2}\right) \\
E_{x}\left(\omega_{1}\right) E_{z}\left(\omega_{2}\right)+E_{z}\left(\omega_{1}\right) E_{x}\left(\omega_{2}\right) \\
E_{x}\left(\omega_{1}\right) E_{y}\left(\omega_{2}\right)+E_{y}\left(\omega_{1}\right) E_{x}\left(\omega_{2}\right)
\end{array}\right]
$$

Similarly, we can also write Eqn. (2.19) (SHG) in terms of a matrix equation as

$$
\left[\begin{array}{l}
P_{x}(2 \omega) \\
P_{y}(2 \omega) \\
P_{z}(2 \omega)
\end{array}\right]=2\left[\begin{array}{llllll}
d_{11} & d_{12} & d_{13} & d_{14} & d_{15} & d_{16} \\
d_{21} & d_{22} & d_{23} & d_{24} & d_{25} & d_{26} \\
d_{31} & d_{32} & d_{33} & d_{34} & d_{35} & d_{36}
\end{array}\right]\left[\begin{array}{c}
E_{x}(\omega)^{2} \\
E_{y}(\omega)^{2} \\
E_{z}(\omega)^{2} \\
2 E_{y}(\omega) E_{z}(\omega) \\
2 E_{x}(\omega) E_{z}(\omega) \\
2 E_{x}(\omega) E_{y}(\omega)
\end{array}\right]
$$

Before we discuss Eqn. (2.21) further in terms of imposing a specific crystal symmetry, we will digress and discuss a material's linear response to an applied optical (electric) field.

\subsection{Anisotropic materials}

\subsubsection{Dielectric tensor $\varepsilon_{i j}$}

When an electric field is applied to a material, the dielectric tensor $\left(\varepsilon_{i j}\right)$ relates the material's linear response $(\vec{D})$ to the applied electric field $(\vec{E})$. The dielectric tensor is an important concept in the study of nonlinear optics because it is closely related to $\chi^{(1)}$ and 
the linear refractive index [5]. Anisotropy in a material's dielectric tensor (index of refraction) is the basis for constructing the index ellipsoid [8] and can be utilized to produce efficient nonlinear interactions. The dielectric tensor (i.e. the relation between $\vec{D}$ and $\vec{E}$ ) can be derived from first principles using Maxwell's equations.

Maxwell's equations in c.g.s. units are

$$
\begin{array}{ll}
\nabla \times \overrightarrow{\mathrm{E}}=-\frac{1}{\mathrm{c}} \frac{\partial}{\partial \mathrm{t}}(\mu \overrightarrow{\mathrm{H}}) & \nabla \times \overrightarrow{\mathrm{H}}=\frac{1}{\mathrm{c}} \frac{\partial \overrightarrow{\mathrm{D}}}{\partial \mathrm{t}}+\frac{4 \pi}{\mathrm{c}} \overrightarrow{\mathrm{j}}_{\mathrm{f}} \\
\nabla \cdot \overrightarrow{\mathrm{D}}=4 \pi \rho_{\mathrm{f}} & \nabla \cdot \overrightarrow{\mathrm{B}}=0
\end{array}
$$

with the constitutive equations

$$
\overrightarrow{\mathrm{D}}=\overrightarrow{\mathrm{E}}+4 \pi \overrightarrow{\mathrm{P}} \quad \overrightarrow{\mathrm{H}}=\overrightarrow{\mathrm{B}}-4 \pi \overrightarrow{\mathrm{M}}
$$

We will now focus on the first constitutive equation involving $\vec{D}$ and assume that electrical anisotropy will be encountered. We use the first term on the right hand side of Eqn. (2.1) to write $P_{i}{ }^{(1)}=\chi_{i j}{ }^{(1)} E_{j}$, where $P_{i}{ }^{(1)}$ is the linear polarization and $\chi_{i j}{ }^{(1)}$ is the linear electric susceptibility. Inserting this relation for $\vec{P}$ into the expression for $\vec{D}$ given in Eqn. (2.26) we obtain

$$
D_{i}=\varepsilon_{i j} E_{j}
$$

where $\varepsilon_{i j} \equiv 1+4 \pi \chi_{i j}{ }^{(1)}$ [9] and $i$ and $j$ designate cartesian components. $\varepsilon_{i j}$ is called the dielectric tensor. We can write Eqn. (2.27) in matrix form as 


$$
\left[\begin{array}{l}
D_{x} \\
D_{y} \\
D_{z}
\end{array}\right]=\left[\begin{array}{lll}
\varepsilon_{x x} & \varepsilon_{x y} & \varepsilon_{x z} \\
\varepsilon_{y x} & \varepsilon_{y y} & \varepsilon_{y z} \\
\varepsilon_{z x} & \varepsilon_{z y} & \varepsilon_{z z}
\end{array}\right]\left[\begin{array}{l}
E_{x} \\
E_{y} \\
E_{z}
\end{array}\right]
$$

A material that has isotropic electrical properties has a dielectric tensor that in the principal axis system is diagonalized with components that are all equal (i.e. $\varepsilon_{\mathrm{xx}}=\varepsilon_{\mathrm{yy}}=$ $\varepsilon_{\mathrm{zz}}$ ) [9]. A material that has anisotropic electrical properties (i.e. electrical properties that vary along different directions in the material) has a dielectric tensor that in the principal axis system is diagonalized but with components that are in general not equal (i.e. $\varepsilon_{\mathrm{xx}} \neq$ $\left.\varepsilon_{\mathrm{yy}} \neq \varepsilon_{\mathrm{zz}}\right)$

We can relate $\varepsilon_{\mathrm{ij}}$ for an isotropic material to the linear refractive index (n) through the wave velocity $\left(v=\frac{c}{n}\right)$ and Eqns. (2.25) and (2.26) [9]. The result is

$$
\mathrm{n}^{2}=\varepsilon=1+4 \pi \chi
$$

where we have used $\varepsilon_{i j}=\varepsilon$ and $\chi_{i j}=\chi$ since for an isotropic material $\varepsilon_{i j}\left(\chi_{i j}\right)$ has only diagonal components that are all equal.

An anisotropic material will, according to Eqns. (2.28) and (2.29), have refractive index values that vary depending on the direction chosen in the material [8]. Materials that have more than one value for the index of refraction are called birefringent. A consequence of birefringence is that for a particular input polarization, the material can respond in orthogonal directions since $\varepsilon_{i j}$ is a tensor. In other words, in general $\vec{E}$ and $\vec{D}$ will not be in the same direction in birefringent materials. Most materials that are 
naturally birefringent are crystalline and so the following discussion will be limited to crystalline materials.

\subsubsection{Index ellipsoid}

A very useful tool for understanding and utilizing birefringent crystals is the index ellipsoid. The index ellipsoid is a geometric construction that can be used to find the two allowed polarization directions and the corresponding refractive indices for an arbitrary direction of propagation through a birefringent crystal [8]. In order to develop the index ellipsoid, we will consider the electromagnetic energy density and the Poynting vector for a wave (optical field) propagating in a birefringent crystal. Equations for calculating the electric field energy density $\left(W_{e}\right)$, the magnetic field energy density $\left(W_{m}\right)$, and the Poynting vector $(\overrightarrow{\mathrm{S}})$ are given by

$$
\begin{gathered}
W_{e}=\frac{1}{8 \pi}(\overrightarrow{\mathrm{E}} \cdot \overrightarrow{\mathrm{D}})=\frac{1}{8 \pi} \mathrm{E}_{\mathrm{j}} \varepsilon_{\mathrm{jk}} \mathrm{E}_{\mathrm{k}} \\
\mathrm{W}_{\mathrm{m}}=\frac{1}{8 \pi}(\overrightarrow{\mathrm{B}} \cdot \overrightarrow{\mathrm{H}})=\frac{1}{8 \pi} \mu \mathrm{H}^{2} \\
\overrightarrow{\mathrm{S}}=\frac{\mathrm{c}}{4 \pi}(\overrightarrow{\mathrm{E}} \times \overrightarrow{\mathrm{H}})
\end{gathered}
$$

respectively. The net power flow into a unit volume is given by

$$
\nabla \cdot \overrightarrow{\mathrm{S}}=\frac{\mathrm{c}}{4 \pi} \nabla \cdot(\overrightarrow{\mathrm{E}} \times \overrightarrow{\mathrm{H}})
$$

Now by taking the dot product of $\vec{E}$ with the curl of $\overrightarrow{\mathrm{H}}$ and the dot product of $\overrightarrow{\mathrm{H}}$ with the curl of $\vec{E}$ in Eqn. (2.25) and using a vector identity [6] we find 


$$
\nabla \cdot \overrightarrow{\mathrm{S}}=\frac{1}{4 \pi}\left(\mathrm{E}_{\mathrm{k}} \varepsilon_{\mathrm{kl}} \frac{\partial \mathrm{E}_{\mathrm{l}}}{\partial \mathrm{t}}+\overrightarrow{\mathrm{H}} \cdot \frac{\partial \overrightarrow{\mathrm{H}}}{\partial \mathrm{t}}\right)
$$

where Eqn. (2.27) has also been used. The second term on the right-hand side of Eqn. (2.34) is the rate of change of the magnetic energy per unit volume. The first term on the right-hand side of Eqn. (2.34) is the rate of change of the electric energy per unit volume only if the dielectric tensor $\left(\varepsilon_{\mathrm{ij}}\right)$ is symmetric, i.e.

$$
\varepsilon_{\mathrm{ij}}=\varepsilon_{\mathrm{ji}}
$$

This symmetry requirement reduces the number of independent components of $\varepsilon_{\mathrm{ij}}$ to six. Writing out the expression for the stored electric energy density (Eqn. (2.30)) and using the symmetry of $\varepsilon_{\mathrm{ij}}$ we find

$$
W_{c}=\frac{1}{8 \pi}\left(\varepsilon_{x x} E_{x}^{2}+\varepsilon_{y y} E_{y}^{2}+\varepsilon_{z z} E_{z}^{2}+2 \varepsilon_{y z} E_{y} E_{z}+2 \varepsilon_{x z} E_{x} E_{z}+2 \varepsilon_{x y} E_{x} E_{y}\right)
$$

$\mathrm{W}_{\mathrm{e}}$ is a positive definite quantity because it represents energy. Therefore geometrically Eqn. (2.36) defines the surface of an ellipsoid. The axes of the ellipsoid can be found by rotating to a new orthogonal coordinate system that diagonalizes $\varepsilon_{\mathrm{ij}}$, i.e.,

$$
\left[\begin{array}{l}
D_{x} \\
D_{y} \\
D_{z}
\end{array}\right]=\left[\begin{array}{ccc}
\varepsilon_{x} & 0 & 0 \\
0 & \varepsilon_{y} & 0 \\
0 & 0 & \varepsilon_{z}
\end{array}\right]\left[\begin{array}{l}
E_{x} \\
E_{y} \\
E_{z}
\end{array}\right]
$$

Note that since we are considering an anisotropic crystal, in general $\varepsilon_{\mathrm{x}} \neq \varepsilon_{\mathrm{y}} \neq \varepsilon_{\mathrm{z}}$. The expression for $\mathrm{W}_{\mathrm{e}}$ in Eqn. (2.36) in this new coordinate system becomes 


$$
W_{e}=\frac{1}{8 \pi}\left(\varepsilon_{x} E_{x}^{2}+\varepsilon_{y} E_{y}^{2}+\varepsilon_{z} E_{z}^{2}\right)
$$

The axes of the coordinate system that diagonalizes $\varepsilon_{\mathrm{ij}}$ are called the principal dielectric axes. The principal dielectric axes are special in the sense that when $\overrightarrow{\mathrm{E}}$ coincides in direction to one of these axes, $\vec{E}$ and $\vec{D}$ will be parallel. When $\vec{E}$ is not along one of the principal dielectric axes we see from Eqn. (2.37) that $\vec{E}$ and $\vec{D}$ will point in different directions. As a consequence, the wavevector $\left(\vec{k}=\frac{c}{\omega n H^{2}}(\vec{H} \times \vec{D})\right)$ and the Poynting vector $\left(\overrightarrow{\mathrm{S}}=\frac{c}{4 \pi}(\overrightarrow{\mathrm{E}} \times \overrightarrow{\mathrm{H}})\right)$ will not be collinear. Therefore when a randomly polarized beam enters a crystal its two allowed orthogonal polarizations [8] will in general propagate in two different directions and the beam will split into two beams. This is known as double refraction [10]. Another term used to describe double refraction is "walk-off" which refers to how the two orthogonal polarizations separate from each other as they propagate through the crystal and produce two beams [11].

Let's express Eqn. (2.38) in terms of the components of $\vec{D}$ (i.e. $D_{x}=\varepsilon_{x} E_{x}$, etc.) and use $n_{i} \equiv \sqrt{\mu \varepsilon_{i}}[6]$ (this definition for the $n_{i}$ 's defines the principal indices of refraction). Substituting into Eqn. (2.38) we obtain

$$
W_{e}=\frac{1}{8 \pi}\left(\frac{D_{x}^{2}}{n_{x}^{2}}+\frac{D_{y}^{2}}{n_{y}^{2}}+\frac{D_{z}^{2}}{n_{z}^{2}}\right)
$$

Letting $x=D_{\mathrm{x}} / \sqrt{8 \pi \mathrm{W}_{\mathrm{e}}}$, etc. in Eqn. (2.39) we find 


$$
\frac{x^{2}}{\mathrm{n}_{\mathrm{x}}^{2}}+\frac{y^{2}}{\mathrm{n}_{\mathrm{y}}^{2}}+\frac{z^{2}}{\mathrm{n}_{\mathrm{z}}^{2}}=1
$$

Eqn. (2.40) represents an ellipsoid with its axes in the $x, y$, and $z$ directions. It is known as the index ellipsoid or the optical indicatrix. A basic index ellipsoid is shown in Fig. 2.1. The directions $x, y$, and $z$ in Fig. 2.1 are called either the principal dielectric axes or

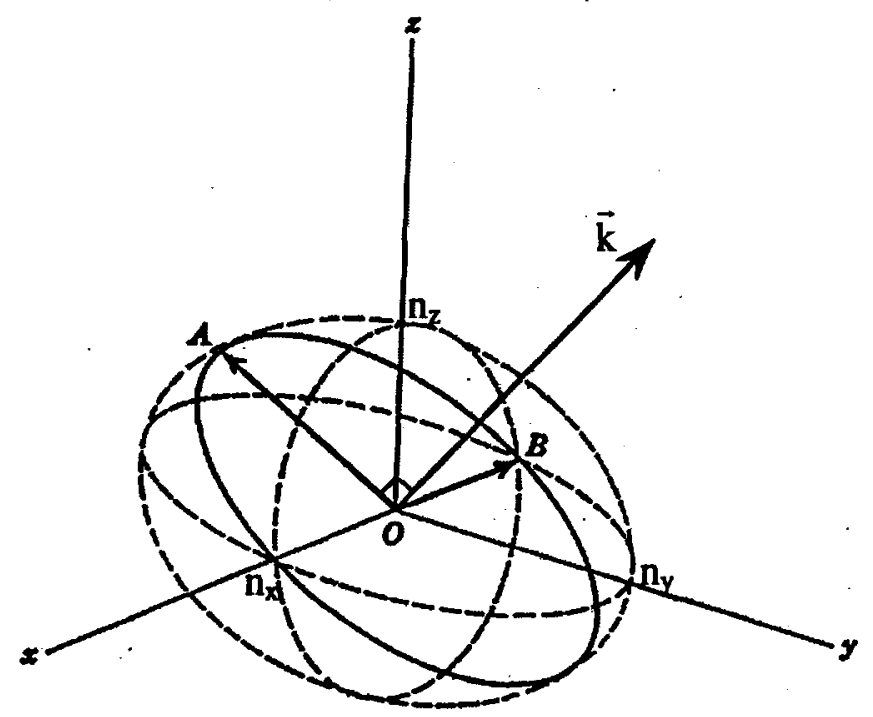

Figure 2.1. The index ellipsoid. The principal refractive indices are labeled $\left(n_{x}, n_{y}\right.$, and $\mathbf{n}_{\mathbf{z}}$ ) and $A$ and $B$ are the two allowed orthogonal polarizations for the given wavevector $\vec{k}$ [6].

the dielectric directions. The lengths of the axes of the index ellipsoid (along $x, y$, and $z$ ) shown in Fig. 2.1 are equal to the values of the principal refractive indices $\left(n_{x}, n_{y}, n_{z}\right)$. The planes formed by the principal axes are called the principal planes.

We will now describe how the index ellipsoid can be used to find the two allowed polarization directions and the corresponding refractive indices for an arbitrary direction of propagation. This will be an operational description given without proof. The formal proof of why this works is given by Born and Wolf [8]. The basic technique is shown in 
Fig. 2.1. Through the center of the ellipsoid we draw a plane perpendicular to the direction of propagation $(\vec{k})$. The intersection of this plane and the ellipsoid is in general an ellipse (ellipse with solid line in Fig. 2.1). The two axes of the ellipse are parallel to the two allowed polarization directions and the length of each axis is equal to the value of the refractive index in that direction. The exact shape of the index ellipsoid for a particular crystal depends on the relationship between $n_{x}, n_{y}$, and $n_{z}$ (i.e. $n_{x} \neq n_{y}$, etc.) which is determined by the crystal's spatial symmetry.

\subsubsection{COB crystals}

If the lengths of all three axes of the optical indicatrix are equal the crystal has only one refractive index and is referred to as isotropic. If any axes of the optical indicatrix are not equal the crystal is termed anisotropic. There are two types of anisotropic crystals encountered in nature. If two axes of the optical indicatrix are equal, the crystal is termed unaxial. The term unaxial is used because in this case the crystal has one optic axis that is perpendicular to the plane formed by the two equal axes of the indicatrix. (An optic axis is defined as the wavevector direction that has a refractive index value that is independent of the direction of polarization, or in other words, it is the wavevector direction perpendicular to a circular cross-section of the optical indicatrix.) If all three axes of the indicatrix are unequal (three different values for the principal refractive indices), the crystal is called biaxial. The term biaxial is used because in this case the crystal has two primary optic axes [8]. For a biaxial crystal with $n_{x}<n_{y}<n_{z}$, the two optic axes lie in the $x z$ principal plane [10]. If $n_{y}$ is closer in value to $n_{x}$ the crystal is called positive biaxial and if $n_{y}$ is closer to $n_{z}$ the crystal is called negative biaxial [10]. For a positive biaxial crystal, the $\mathrm{z}$-axis is the acute bisector of the angle between the two 
optic axes. For a negative biaxial crystal, the $x$-axis is the acute bisector of the angle between the two optic axes. Fig. 2.2 shows the optical indicatrix for a negative biaxial crystal. In the following sections, we will discuss properties and interactions primarily for polarizations that are either along principal axes or that lie in principal planes. The

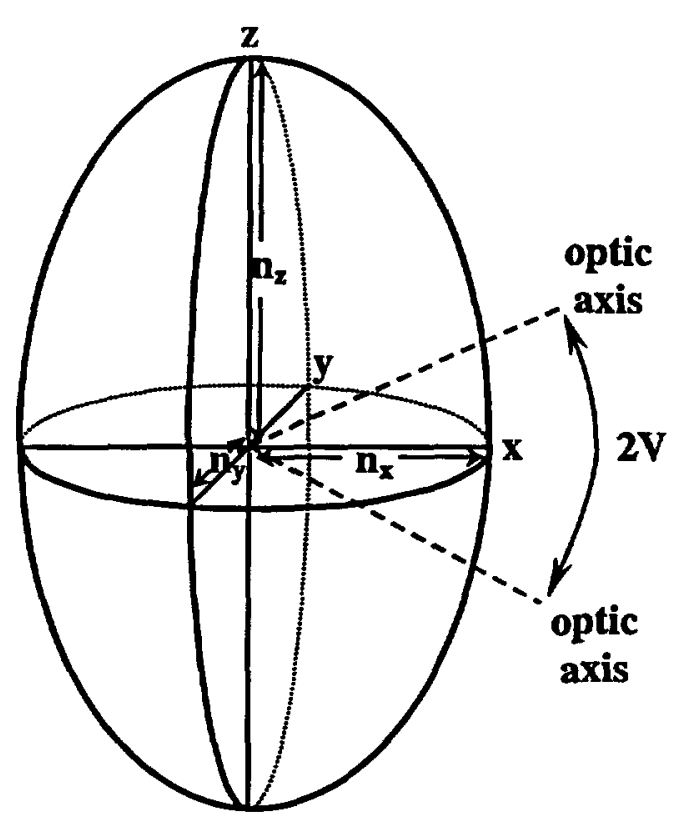

Figure 2.2. Optical indicatrix for a negative biaxial crystal where $n_{x}<n_{y}<n_{z}$. The $x, y$, and $z$-axes are the crystal's dielectric axes and $n_{x}, n_{y}$, and $n_{z}$ are the principal refractive indices. Note that the optic axes lie in the $x z$ plane where the $x$ dielectric axis is the acute bisectrix and $\mathrm{V}$ is the optic angle [10].

refractive index for wavevectors $(\overrightarrow{\mathrm{k}})$ lying for instance in the $\mathrm{xz}$ principal plane, can be calculated from

$$
n_{x z}=\frac{n_{x} n_{z}}{\left(n_{x}^{2} \cos ^{2} \phi+n_{z}^{2} \sin ^{2} \phi\right)^{\frac{1}{2}}}
$$

where $n_{x}$ and $n_{z}$ are principal refractive indices and $\phi$ is measured from the $x$-axis in the $x z$ plane. The refractive index for wavevectors oriented in the other principal planes can 
be calculated similarly by using the appropriate pair of indices and knowing from which axis the angle is measured.

Many different conventions for specifying directions in biaxial crystals can be found in the literature [12]. Our convention for specifying directions in biaxial crystals will be in terms of the Euler angles $\phi$ and $\theta$ as measured from the principal dielectric planes. The angle $\phi$ will be measured from the acute bisectrix (Fig. 2.2) within the plane containing the acute bisectrix and the optic axis. The angle $\theta$ will be measured from the $y$ dielectric axis in the xy plane. For uniaxial crystals, our convention will be that $\theta$ is measured from the optic axis and $\phi$ is measured from the $\mathrm{x}$ dielectric axis. Another convention we will use concerns labeling of the principal refractive indices for biaxial crystals. We will always label the smallest principal refractive index of a biaxial crystal with $n_{x}$ and the largest principal refractive index with $\mathbf{n}_{\mathbf{z}}$.

Whether a crystal is isotropic or anisotropic, and in the anisotropic case, whether it is uniaxial or biaxial, is determined by the crystal's point group symmetry. Crystals with cubic symmetry are isotropic. Crystals with trigonal, tetragonal, or hexagonal symmetry are uniaxial. Crystals with orthorhombic, monoclinic, or triclinic symmetry are biaxial. A crystal's specific point group is determined by the crystal's atomic structure and can be described by 3 crystallographic axes (a,b, and $c)$ and 3 angles $(\alpha, \beta$, and $\gamma$ ). For the monoclinic structure, the three crystallographic axes a, b, and c are not mutually orthogonal [10]. The directions of the crystallographic axes are typically determined using X-ray diffraction and are generally different in direction from the principal dielectric axes.

The $\mathrm{COB}$ crystals have been determined using X-ray diffraction at LLNL to be 
monoclinic crystals belonging to the $\mathrm{m}$ symmetry class. The convention used for the single mirror plane is that it is perpendicular to the $b$ crystallographic axis. The COB crystals have been determined by Ref. [3] to be negative biaxial crystals. The crystal structure for GdCOB is shown in Fig. 2.3. The unit cell constants for GdCOB were

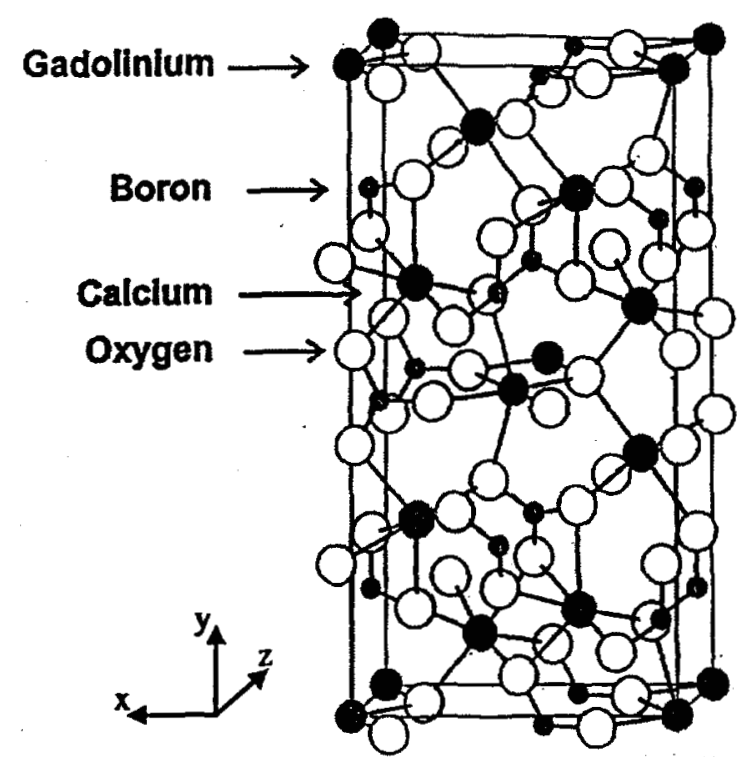

Figure 2.3. Crystal structure for $\mathrm{GdCOB}$ [3].

determined by Ref. [3] and found to have values of $a=0.8095 \mathrm{~nm}, b=1.6018 \mathrm{~nm}$, and $c$ $=0.3558 \mathrm{~nm}$. The relative directions of the dielectric axes and the crystallographic axes for GdCOB were also determined by Ref. [3] and are shown in Fig. 2.4. The ac crystallographic plane is parallel to the $x z$ principal plane. The $b$ crystallographic axis is parallel to the $y$ dielectric axis, the $c$-axis is at $15^{\circ}$ to the $x$-axis, and the a-axis is at $26^{\circ}$ to the $\mathrm{z}$-axis as shown.

We now return to the discussion of the $d_{i l}$ matrix (Eqn. (2.21)) and will give its form for the COB crystals. The form of the nonlinear susceptibility tensor, or equivalently the $d_{i l}$ tensor, is constrained by the symmetry properties of the nonlinear optical medium. The $d_{i l}$ matrices have been previously determined for all the known symmetry groups and 


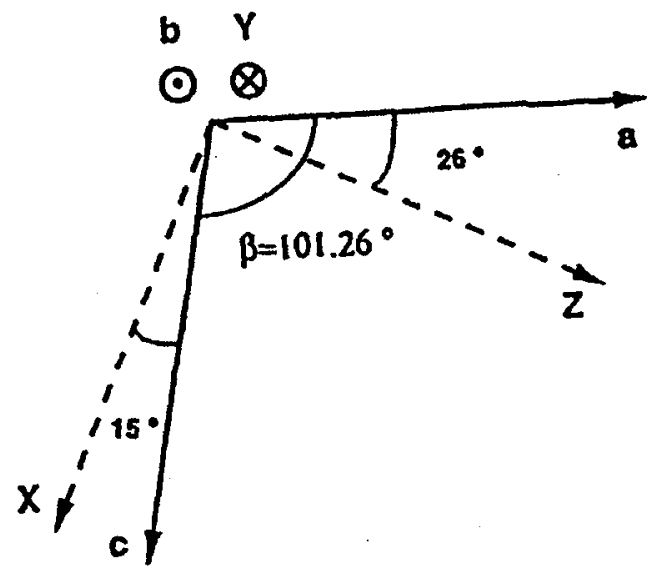

Figure 2.4. Relative orientation of the dielectric axes $(x, y, z)$ to that of the crystallographic axes $(a, b, c)$ for $\mathrm{GdCOB}[3]$.

can be looked-up in many books $[5,7,13]$. The COB crystals are in the monoclinic crystal class with point group $m$ symmetry with the convention that $m \perp b$. Therefore the $d_{i l}$ tensor has the form given in Eqn (2.42) [5]. The symmetry group (m) makes several

$$
d_{i 1} \text { (monoclinic crystal class, point group } m \text { ) }=\left[\begin{array}{cccccc}
d_{11} & d_{12} & d_{13} & 0 & d_{31} & 0 \\
0 & 0 & 0 & d_{32} & 0 & d_{12} \\
d_{31} & d_{32} & d_{33} & 0 & d_{13} & 0
\end{array}\right]
$$

components of the $d_{i 1}$ matrix zero [7] as is also shown in Eqn. (2.42). The form of the $d_{i l}$ matrix in Eqn. (2.42) is what will be used when describing the SHG and SFG interactions in the COB crystals.

\subsection{Second harmonic generation (SHG)}

\subsection{SHG efficiency}

In Chapters 3 and 5, we will present and analyze results from SHG experiments conducted with the COB crystals. These experiments will observe SHG and investigate how the SHG depends on several quantities including wavevector mismatch $(\Delta \mathrm{k})$. 
Therefore we will now discuss the relevant concepts and derive the mathematical expressions that will be necessary to explain and interpret the experimental data in Chapters 3 and 5.

A special case of SFG occurs when both input waves have frequency $\omega$ and the generated wave has frequency $2 \omega$. This is called second harmonic generation (SHG). We would like to know mathematically how the irradiance at $\omega$ is related to the irradiance at $2 \omega$ for SHG. This would allow us to predict the efficiency of our SHG interaction. We will now derive a relationship between the irradiance at $\omega$ and the irradiance at $2 \omega$ that involves, among other parameters, the amplitudes of the interacting waves, the coupling coefficient (d), and the wavevector mismatch $(\Delta \mathrm{k})$.

Starting with Maxwell's equations given in Eqn. (2.25), we will first derive a wave propagation equation that has the nonlinear polarization as a source term. This wave equation will allow us to determine coupled equations between the $1 \omega$ and $2 \omega$ amplitudes for the case of SHG, which we will then solve to determine the $2 \omega$ amplitude as a function of the $1 \omega$ amplitude. If we assume $\overrightarrow{\mathrm{j}}_{\mathrm{f}}=0$ and that the material is nonconducting and nonmagnetic, from Eqn. (2.25) we obtain

$$
\nabla \times \overrightarrow{\mathrm{H}}=\frac{1}{\mathrm{c}} \frac{\partial \overrightarrow{\mathrm{D}}}{\partial \mathrm{t}} \quad \nabla \times \overrightarrow{\mathrm{E}}=-\frac{1}{\mathrm{c}} \frac{\partial}{\partial \mathrm{t}}(\mu \overrightarrow{\mathrm{H}})
$$

with the constitutive relation

$$
\overrightarrow{\mathrm{D}}=\varepsilon \overrightarrow{\mathrm{E}}+4 \pi \overrightarrow{\mathrm{P}}_{\text {nonlinear }}
$$

In Eqn. (2.44), the linear polarization is included in $\varepsilon$ and $\overrightarrow{\mathrm{P}}_{\text {nonlinear }}$ is the second-order 
nonlinear polarization. If we take the curl of the curl of $\vec{E}$ given in Eqn. (2.43), we find using the curl of $\overrightarrow{\mathrm{H}}$, the vector identity $\overrightarrow{\mathrm{A}} \times \overrightarrow{\mathrm{B}} \times \overrightarrow{\mathrm{C}}=\overrightarrow{\mathrm{B}}(\overrightarrow{\mathrm{A}} \cdot \overrightarrow{\mathrm{C}})-\overrightarrow{\mathrm{C}}(\overrightarrow{\mathrm{A}} \cdot \overrightarrow{\mathrm{B}})$, and $\nabla \cdot \overrightarrow{\mathrm{E}}=0$ for plane waves [5] that

$$
\nabla^{2} \overrightarrow{\mathrm{E}}=\frac{1}{\mathrm{c}^{2}} \frac{\partial^{2}}{\partial \mathrm{t}^{2}} \varepsilon \overrightarrow{\mathrm{E}}+\frac{4 \pi}{\mathrm{c}^{2}} \frac{\partial^{2}}{\partial \mathrm{t}^{2}} \overrightarrow{\mathrm{P}}_{\text {nonlinear }}
$$

This is the driven nonlinear wave equation and we will use it to find coupled equations that relate the $1 \omega$ and $2 \omega$ amplitudes for SHG.

We will now restrict the problem to propagation along one-dimension (i.e. in the $\mathrm{z}$ direction) for simplicity. We will use the form for $\overrightarrow{\mathrm{E}}$ given in Eqn. (2.6) and the forms for $\overrightarrow{\mathrm{P}}$ given in Eqns. (2.7), (2.15), and (2.19) to write our one-dimensional fields. Therefore, with $\omega_{2}=2 \omega_{1}$ (note this is a slightly different notation than was used in section 2.2), the amplitudes of the two interacting electric fields can be written as

$$
\begin{aligned}
& E_{1}(z, t)=E_{1}\left(\omega_{1}\right) e^{-i\left(\omega_{1} t-k_{1} z\right)} \\
& E_{2}(z, t)=E_{2}\left(\omega_{2}\right) e^{-i\left(\omega_{2} t-k_{2} z\right)}
\end{aligned}
$$

Likewise the polarization amplitudes can be written as

$$
\begin{gathered}
P_{1}(z, t)=4 d E_{2}\left(\omega_{2}\right) E_{1}^{*}\left(\omega_{1}\right) e^{-i\left(\left(\omega_{2}-\omega_{1}\right) t-\left(k_{2}-k_{1}\right) z\right)} \\
P_{2}(z, t)=2 \mathrm{dE}_{1}^{2}\left(\omega_{1}\right) e^{-2 i\left(\omega_{1} 1-k_{1} z\right)}
\end{gathered}
$$

The nonlinear coupling coefficient (d) in Eqn. (2.47) is written in a completely general form. (As will be discussed shortly, the form for $d$ is not necessarily a single component 
of the $\mathrm{d}_{\mathrm{il}}$ tensor but in general is dependent on the symmetry of the crystal and the geometry of the interaction.) Note that the subscripts on the amplitudes in Eqns. (2.46) and (2.47) have the following meanings $1 \Rightarrow \omega_{1}$ and $2 \Rightarrow \omega_{2}=2 \omega_{1}$. Also, note that $k_{1}=$ $2 \pi n\left(\omega_{1}\right) / \lambda_{1}$ and $k_{2}=2 \pi n\left(\omega_{2}\right) / \lambda_{2}=4 \pi n\left(\omega_{2}\right) / \lambda_{1}$

We will now derive the coupled amplitude equations for $E_{1}$ and $E_{2}$ by substituting Eqns. (2.46) and (2.47) into the wave equation (Eqn. (2.45)). If we assume that the conversion efficiency is small, then the field amplitudes will change very slowly in the $\mathrm{z}$ direction and we can make use of the slowly-varying envelope approximation. The slowly-varying envelope approximation is stated mathematically by

$$
\left|\frac{d^{2} E_{i}}{d z^{2}}\right| \ll\left|k \frac{d E_{i}}{d z}\right| \text { (for } i=1 \text { and 2) }
$$

Physically, Eqn. (2.48) requires that the change in the amplitude with $z$ occurs only over distances much larger than an optical wavelength. The slowly-varying envelope approximation therefore allows us to ignore the second-order derivatives in our coupled equations. Making the substitutions into the wave equation and using Eqn. (2.48) and $k_{i}^{2}$ $=\omega_{i}{ }^{2} \varepsilon / c^{2}$ (for $i=1$ and 2) [5], we obtain the following pair of coupled equations

$$
\begin{aligned}
& \frac{\partial \mathrm{E}_{1}}{\partial \mathrm{z}}=\frac{8 \pi i \omega_{1}^{2} \mathrm{dE}_{1}^{*} \mathrm{E}_{2}}{\mathrm{k}_{1} \mathrm{c}^{2}} \mathrm{e}^{-\mathrm{i} \Delta \mathrm{kz}} \\
& \frac{\partial \mathrm{E}_{2}}{\partial \mathrm{z}}=\frac{16 \pi i \omega_{1}^{2} \mathrm{dE}_{1}^{2}}{\mathrm{k}_{2} \mathrm{c}^{2}} \mathrm{e}^{\mathrm{i} \Delta \mathrm{kz}}
\end{aligned}
$$

where $\omega_{2}=2 \omega_{1}$ and the wavevector mismatch $\Delta k=\left(2 k_{1}-k_{2}\right)$. The general solution to Eqns. (2.49) and (2.50) is difficult, however, if we assume that the generated $\omega_{2}$ signal is 
small and therefore the input field at $\omega_{1}$ is essentially constant (i.e. $E_{1} \neq E_{1}(z)$ ), we can integrate Eqn. (2.50) from $z=0$ to $z=L$ (here $L$ is the length of the crystal or the interaction length) and use $\omega_{1}=2 \pi \mathrm{c} / \lambda_{1}, \mathrm{k}_{2}=2 \pi \mathrm{n}\left(\omega_{2}\right) / \lambda_{2}$, and $\lambda_{2}=\lambda_{1} / 2$ to obtain

$$
E_{2}=\frac{16 \pi^{2} d E_{1}^{2}}{\Delta k \lambda_{1} n\left(\omega_{2}\right)}\left(e^{i \Delta k L}-1\right)
$$

We would like to express this result as a real quantity and in terms of the irradiances, $I_{i}=$ $\left(n\left(\omega_{i}\right) c / 2 \pi\right)\left|E_{i}\right|^{2}$. So converting to irradiances and also using the identity $2 \sin ^{2} A=1-$ $\cos 2 \mathrm{~A}$ we find the irradiance at $\omega_{2}\left(\mathrm{I}_{2}\right)$ to be given by

$$
I_{2}=\frac{512 \pi^{5} d^{2} I_{1}^{2} L^{2}}{\lambda_{1}^{2} n\left(\omega_{2}\right) n\left(\omega_{1}\right)^{2} c}\left(\frac{\sin \left(\frac{\Delta k L}{2}\right)}{\frac{\Delta k L}{2}}\right)^{2}
$$

We define the SHG conversion efficiency as

$$
\eta_{\text {eff }} \equiv \frac{I_{2}}{I_{1}}
$$

Eqns. (2.52) and (2.53) allows us to write down $\eta_{\text {eff }}$ for the case of perfect phasematching $(\Delta k=0)$ as

$$
\eta_{\text {eff }} \equiv \frac{I_{2}}{I_{1}}=\frac{512 \pi^{5} d^{2} I_{1} L^{2}}{\lambda_{1}^{2} n\left(\omega_{2}\right) n\left(\omega_{1}\right)^{2} c}
$$

Note that the SHG efficiency in Eqn. (2.54) depends on the $1 \omega$ irradiance $\left(I_{1}\right)$. 


\subsubsection{Phasematching}

The term $\left(\frac{\sin \left(\frac{\Delta \mathrm{kL}}{2}\right)}{\frac{\Delta \mathrm{kL}}{2}}\right)^{2}$ in Eqn. (2.52) is a measure of the phase mismatch between the applied $1 \omega$ wave $\left(\omega_{1}\right)$ and the generated $2 \omega$ wave $\left(\omega_{2}\right)$. Typically, due to a material's dispersion (i.e. $\left.n\left(\omega_{2}\right) \neq n\left(\omega_{1}\right)\right) \Delta k \neq 0(k=2 \pi n / \lambda)$ and therefore the $2 \omega$ signal is small. If $\Delta k$ can be made $\approx 0$, then the $1 \omega$ and $2 \omega$ waves will be in phase and the $1 \omega$ wave will be efficiently converted producing a large $2 \omega$ signal. Arranging to have $\Delta k=0$ is called phasematching. Phasematching is therefore conservation of linear momentum $(p=\hbar k)$ by the nonlinear interaction. Stated mathematically for our one-dimensional case, phasematched SHG occurs when

$$
\Delta k=\left(2 k_{1}-k_{2}\right)=0 \quad \text { (Conservation of momentum) }
$$

Since the nonlinear interactions we are considering are parametric interactions (i.e. the state of the material is unchanged by the interaction [5]), energy will also be conserved. So a secondary condition that must be satisfied by the nonlinear interaction is conservation of energy. Conservation of energy can be expressed as

$$
\omega_{2}=\omega_{1}+\omega_{1} \quad \text { (Conservation of energy) }
$$

In order to achieve efficient conversion and a maximum $2 \omega$ signal, we want a phasematched situation $(\Delta k=0)$. The condition for phasematching in Eqn. (2.55) implies that $2 k_{1}=k_{2}$ or $n\left(\omega_{1}\right)=n\left(\omega_{2}\right)$. One method of achieving $n\left(\omega_{1}\right)=n\left(\omega_{2}\right)$ is to utilize the birefringence of an anisotropic crystal. In an anisotropic crystal, or most any material for 
that matter, the indices of refraction depend on the wavelength of the light. This is called dispersion and is illustrated in Fig. 2.5. The dependence of the refractive indices on

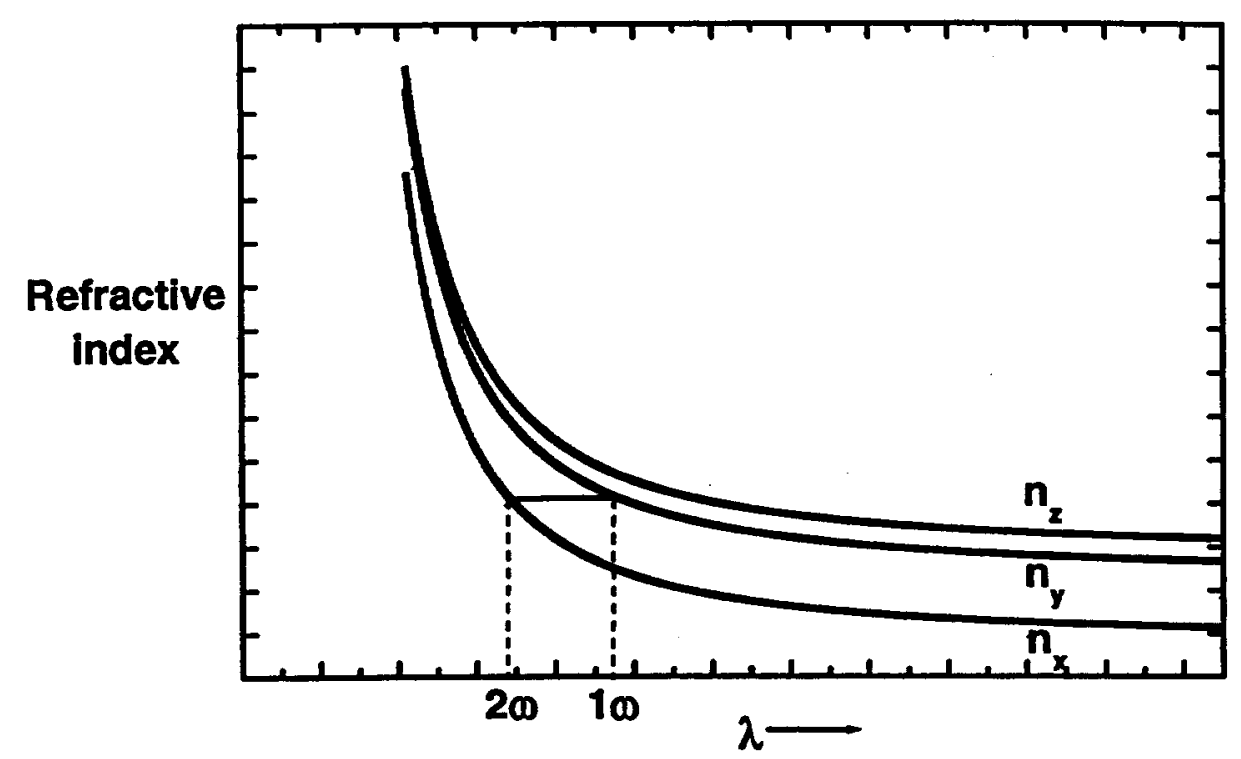

Figure 2.5. Principal refractive indices as a function of wavelength for a negative biaxial crystal showing a pair of phasematched SHG wavelengths for propagation down the $z$ dielectric axis.

wavelength as shown in Fig. 2.5 is usually expressed mathematically in terms of Sellmeier equations [8] which we will describe shortly. Fig. 2.5 also illustrates the basics of phasematching. As shown, a pair of $1 \omega$ and $2 \omega$ waves have equal refractive indices which makes $\Delta k=0$ and therefore the SHG process is phasematched. The absolute values for the indices in Fig. 2.5 depend on the propagation direction through the crystal as described for example by Eqn. (2.41). The refractive index values change as the propagation direction changes because different cross-sections of the index ellipsoid are being taken. Often we are interested in phasematching a particular pair of wavelengths and so the angle of propagation must be properly chosen so that the desired wavelengths are phasematched. This is called angle-tuned phasematching. Terhune, et al. [14] and 
Giordmaine [15] originally developed this method of phasematching.

We will study phasematched SHG in the $x z$ and $x y$ plane of the COB crystals. The first piece of information we will need to know in order to determine the phasematched wavelengths is how the principal refractive indices for the COB crystals vary with wavelength, in other words, we need the Sellmeier equations for the COB crystals. For example, a set of Sellmeier equations has been determined for YCOB by Ref. [2] and are given by

$$
\begin{aligned}
& n_{x}=\left(2.81697+\frac{.022418}{\lambda^{2}-.0149216}-7.73709 \times 10^{-5} \lambda^{2}\right)^{\frac{1}{2}} \\
& n_{y}=\left(2.91924+\frac{.0252847}{\lambda^{2}-.0130221}-6.44650 \times 10^{-5} \lambda^{2}\right)^{\frac{1}{2}} \\
& n_{z}=\left(2.95452+\frac{.025835}{\lambda^{2}-.0125276}-7.64814 \times 10^{-5} \lambda^{2}\right)^{\frac{1}{2}}
\end{aligned}
$$

The wavelength value used in these equations is to be expressed in microns $(\mu \mathrm{m})$. These equations can be used to calculate the principal refractive indices for $\mathrm{YCOB}$ for a given wavelength in the range of $0.3-1.3 \mu \mathrm{m}$.

There are two types of phasematching that we will consider: type I and type II. Type I phasematching is where both $1 \omega$ waves are polarized in the same direction and the $2 \omega$ wave is polarized in a perpendicular direction. Type II phasematching is where the two $1 \omega$ waves are polarized in orthogonal directions and the $2 \omega$ wave is polarized parallel to the $1 \omega$ wave in the direction of the lower index [6].

For type I SHG in the $\mathrm{Xz}$ plane of the COB crystals, that is with both $1 \omega$ waves 
polarized along the $y$ dielectric axis and the $2 \omega$ wave polarized in the $x z$ plane, the condition for phasematching is

$$
\Delta k=2 k_{1}-k_{2}=\frac{4 \pi}{\lambda_{1}}\left(n_{y}\left(\omega_{1}\right)-n_{x z}\left(\omega_{2}\right)\right)=0
$$

where $n_{y}\left(\omega_{1}\right)$ is the refractive index along the $y$ dielectric axis at frequency $\omega_{1}$ and $n_{x z}\left(\omega_{2}\right)$ is the refractive index in the direction of the $\omega_{2}$ polarization in the $\mathrm{xz}$ plane as given by Eqn. (2.41). The Sellmeier equations along with Eqn. (2.41) can be used to find the propagation direction that satisfies Eqn. (2.60) for a given pair of wavelengths.

For type II SHG in the xy plane of the COB crystals, the condition for phasematching is

$$
\Delta k=\frac{4 \pi}{\lambda_{1}}\left(n_{x y}\left(\omega_{1}\right)+n_{z}\left(\omega_{1}\right)-n_{x y}\left(\omega_{2}\right)\right)=0
$$

here one of the $1 \omega$ waves is polarized in the xy plane and the other is polarized along the $z$ dielectric axis. The $2 \omega$ wave is generated with its polarization in the $x y$ plane. The refractive index designations are similar to those described for Eqn. (2.60).

As an example, we will calculate how the phasematched wavelength for YCOB depends on the propagation direction for the case of type I SHG in the $\mathrm{xz}$ plane using Eqn. (2.60), the Sellmeier equations given in Eqns. (2.57) - (2.59), and Eqn. (2.41). The calculated phasematched $1 \omega$ wavelengths for $\mathrm{YCOB}$ are shown in Fig. 2.6. The angle of propagation is specified from the $\mathrm{z}$ dielectric axis, which according to our conventions is $\phi-90^{\circ}$. Note that phasematched SHG at $1.064 \mu \mathrm{m}$ occurs at an angle of $32^{\circ}$ from the $\mathrm{z}$ dielectric axis. 


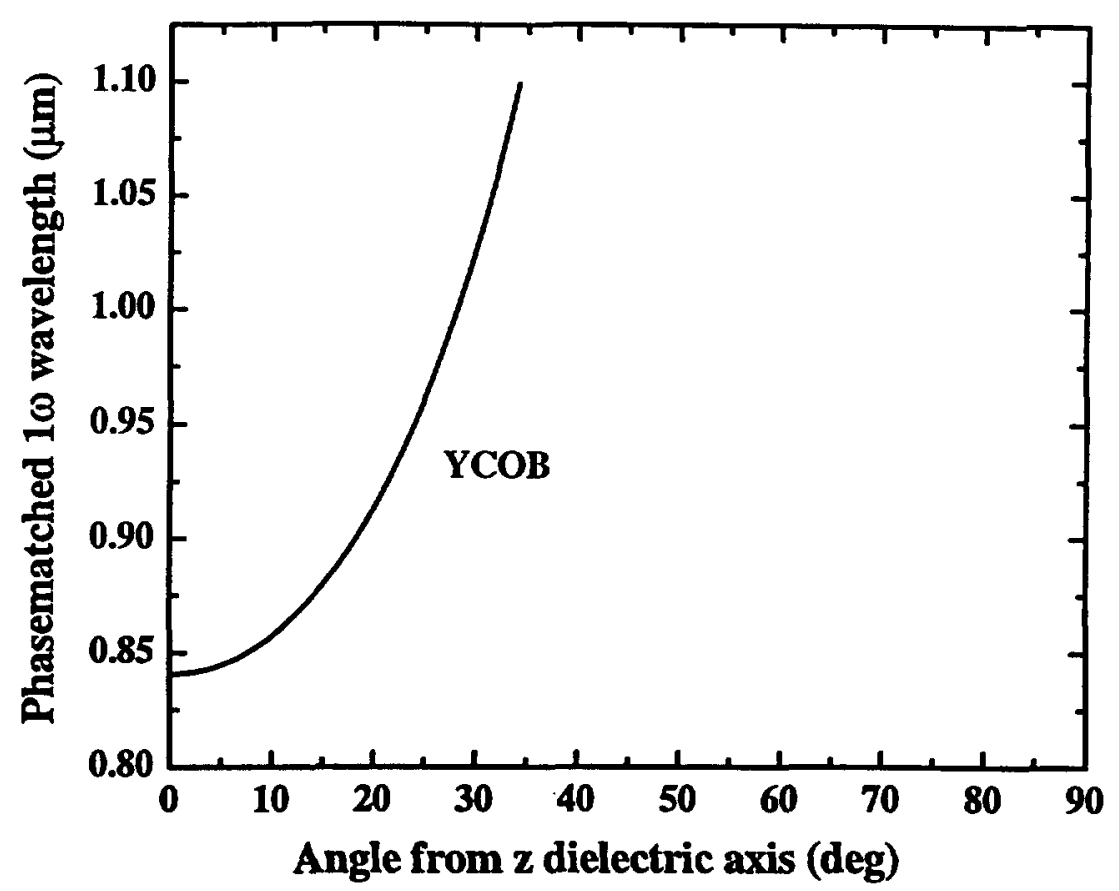

Figure 2.6. Type I phasematched SHG wavelengths for propagation in the $\mathrm{xz}$ plane of $\mathrm{YCOB}$. The angle is measured from the $\mathrm{z}$ dielectric axis in the $\mathrm{xz}$ plane.

\subsection{3 $d_{\text {eff }}$}

As we briefly discussed following Eqn. (2.47), the value of d appearing in Eqn. (2.47) is not necessarily a single component of the $d_{i l}$ tensor. We now want to determine the exact form for the nonlinear coupling coefficient (d) that appears in Eqn. (2.47). We write the $2 \omega$ polarization in a very general form similar to that given in Eqn. (2.47) as

$$
P_{\text {nonlinear }}=\mathrm{dE}_{1} \mathrm{E}_{2}
$$

where $E_{1}$ and $E_{2}$ refer to the two interacting $1 \omega$ waves. Eqn. (2.62) specifies a general coupling coefficient (d), which depends on the components of $d_{i l}$ for the particular crystal symmetry and the geometry of the interaction (propagation direction and type of interaction (I or II)). We now replace $d$ in Eqn (2.62) with $d_{\text {eff }}$ and we refer to it from now on as the effective coupling coefficient (standard nonlinear optics nomenclature). 
We wish to determine $d_{\text {eff }}$ for the case of type I SHG in the $x z$ principal plane of the $\mathrm{COB}$ crystals. We will use YCOB as our example. The general approach [16] will be to first calculate the components of the total nonlinear polarization $(\vec{P})$ generated at $2 \omega$ in terms of the $d_{i l}$ coefficients and the propagation angles using Eqn. (2.14). Once all the components for $\overrightarrow{\mathrm{P}}$ have been found, we will project out the component that is in the correct direction for the interaction (i.e. the component that is in the $x z$ plane perpendicular to direction of propagation). The coefficient that appears in front of the field amplitudes involving the $d_{i l}$ coefficients and the angles will be defined as $d_{\text {eff. }}$ The geometry is shown in Fig. 2.7. For type I SHG in the $x z$ plane of YCOB, with the $1 \omega$ wave polarized along the $y$ dielectric direction, the components of the total nonlinear polarization at $2 \omega$ are found using Eqns. (2.14) and (2.42) to be

$$
\begin{aligned}
& P_{x}=d_{12} E_{y}(1 \omega)^{2} \\
& P_{y}=0 \\
& P_{z}=d_{32} E_{y}(1 \omega)^{2}
\end{aligned}
$$

From these components we want the component that is parallel to the $x z$ plane and the vector $\hat{k}_{\perp}$ shown in Fig. 2.7. We want the component of $\vec{P}_{2 \omega}$ parallel to $\hat{k}_{\perp}$ since this will be the correct direction for the $2 \omega$ polarization for the type I process. The $2 \omega$ wave with this polarization will propagate in the same direction and with the same speed as the $1 \omega$ wave and will therefore be phasematched and grow in irradiance. Since there are only $x$ and $z$ components to the total nonlinear polarization $\left(\overrightarrow{\mathrm{P}}_{2 \omega}\right), \overrightarrow{\mathrm{P}}_{2 \omega}$ lies in the $x z$ plane 


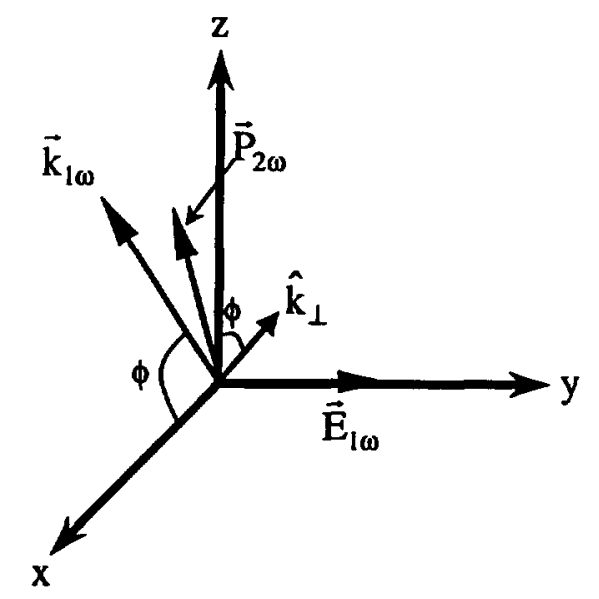

Figure 2.7. Geometry for deriving $d_{\text {eff }}$ for type I SHG in the $x z$ principal plane of YCOB. $x, y$, and $z$ are the principal dielectric directions, $\phi$ is the phasematching angle measured from the $x$ dielectric axis in the $x z$ principal plane, $\vec{E}_{1 \omega}$ is the $1 \omega$ wave amplitude, $\overrightarrow{\mathrm{k}}_{1 \omega}$ is the propagation vector and it lies in the $x z$ plane, and $\vec{P}_{2 \omega}$ is the total $2 \omega$ polarization which is also in the $x z$ plane. $\hat{\mathrm{k}}_{\perp}$ lies in the $x z$ plane perpendicular to $\overrightarrow{\mathrm{k}}_{1 \omega}$ where $\hat{\mathbf{k}}_{\perp}=-\sin \phi \hat{\mathrm{x}}+\cos \phi \hat{\mathbf{z}}$.

Therefore the component of $\overrightarrow{\mathrm{P}}_{2 \omega}$ parallel to $\hat{\mathrm{k}}_{\perp}$ is equal to $\overrightarrow{\mathrm{P}}_{2 \omega} \cdot \hat{\mathrm{k}}_{\perp}$. So we have,

$$
\overrightarrow{\mathrm{P}} \cdot \hat{\mathrm{k}}_{\perp}=\mathrm{d}_{12} \mathrm{E}_{\mathrm{y}}^{2} \sin \phi-\mathrm{d}_{32} \cos \phi \mathrm{E}_{y}^{2}=\left(-\mathrm{d}_{12} \sin \phi+\mathrm{d}_{32} \cos \phi\right) \mathrm{E}_{y}^{2}
$$

The term on the far right of Eqn. (2.64) can be equated with Eqn. (2.62) to determine $d_{\text {eff }}$ for type I SHG in the $\mathrm{xz}$ plane of YCOB as

$$
d_{\text {eff }}=d_{32} \cos \phi-d_{12} \sin \phi \quad \text { for } \phi>V
$$

where $\phi$ is measured from the $x$ dielectric axis and $V$ is the optic angle (Fig. 2.2). Eqn. (2.65) applies to all the COB crystals since they all have the same crystal symmetry.

We would like to comment on the units of $d_{\text {eff }}$ (and $\left.d_{i l}\right)$. As was stated earlier, all of the derivations will be in c.g.s. units unless otherwise noted. The dimensions of the 
effective coefficient $\left(d_{e f f}\right)$ and the tensor coefficients $\left(d_{i l}\right)$ in c.g.s. units are centimeters per stat. volt ( $\mathrm{cm} / \mathrm{statvolt).} \mathrm{In} \mathrm{MKS} \mathrm{units,} \mathrm{which} \mathrm{will} \mathrm{be} \mathrm{the} \mathrm{units} \mathrm{chosen} \mathrm{for} \mathrm{the}$ numerical results in Chapters 3 and $5, \mathrm{~d}_{\text {eff }}$ is expressed in meters per volt $(\mathrm{m} / \mathrm{V})$. The conversion [6] from c.g.s. to MKS units is given by

$$
d\left(\frac{m}{V}\right)=\frac{4 \pi}{3 \times 10^{4}} d\left(\text { esu or } \frac{\mathrm{cm}}{\text { statvolt }}\right)
$$

In MKS units, the second-order nonlinear polarization is in general given by

$$
P=\varepsilon_{0} d E_{1} E_{2}
$$

where $\varepsilon_{0}$ is the permittivity of free space and has a numerical value of $8.8542 \times 10^{-12}$ $\mathrm{C} / \mathrm{Vm})$. If we include $\varepsilon_{0}$ in the definition of $\mathrm{d}$ in Eqn. (2.67), then the conversion between c.g.s. and MKS units (Eqn. (2.66)) becomes

$$
d\left(\frac{C}{V^{2}}\right)=3.71 \times 10^{-15} d\left(\text { esu or } \frac{\mathrm{cm}}{\text { statvolt }}\right)
$$

\subsubsection{Angular, thermal, and spectral sensitivities}

As we have shown, the $2 \omega$ SHG response is dependent on $\Delta \mathrm{k}$ as given by Eqn. (2.52).

The dependence of the $2 \omega$ irradiance on the function $\left(\frac{\sin \left(\frac{\Delta \mathrm{kL}}{2}\right)}{\frac{\Delta \mathrm{kL}}{2}}\right)^{2}$ is commonly referred to as a sinc ${ }^{2}$ response (pronounced "sink-squared"). $\Delta \mathrm{k}$ can be varied or de-tuned from perfect phasematching $(\Delta k=0)$ by many different parameters, the ones we will consider 
are deviation in angle $(\Delta \theta)$, temperature $(\Delta \mathrm{T})$, and wavelength $(\Delta \lambda)$ measured from $\Delta \mathrm{k}=$ 0. We can relate $\Delta \mathrm{k}$ to these detunings using a first-order Taylor series as

$$
\Delta k=\frac{\partial \Delta k}{\partial \theta} d \theta+\frac{\partial \Delta k}{\partial T} d T+\frac{\partial \Delta k}{\partial \lambda} d \lambda
$$

where it is assumed that when taking a particular derivative the other variables are held fixed.

We can define the coefficients involving the partial derivatives in Eqn. (2.69) as specific sensitivities. Therefore, we define the angular sensitivity $\left(\beta_{\theta}\right)$, thermal sensitivity $\left(\beta_{\mathrm{T}}\right)$, and spectral sensitivity $\left(\beta_{\lambda}\right)$ as

$$
\begin{aligned}
& \beta_{\theta} \equiv \frac{\partial \Delta k}{\partial \theta} \quad \text { (angular sensitivity) } \\
& \beta_{T} \equiv \frac{\partial \Delta k}{\partial T} \quad \text { (thermal sensitivity) } \\
& \beta_{\lambda} \equiv \frac{\partial \Delta k}{\partial \lambda} \quad \text { (spectral sensitivity) }
\end{aligned}
$$

These sensitivities along with the crystal length tell us the FWHM of the $\operatorname{sinc}^{2}$ dependence displayed by the $2 \omega$ irradiance as the particular variable de-tunes $\Delta \mathrm{k}$ away from zero. The FWHM of the $\operatorname{sinc}^{2}$ function in Eqn. (2.52) is related to a particular sensitivity by

$$
\mathrm{FWHM}_{\text {sinc }^{2}}=\frac{5.56622951}{\beta_{\text {variable }} \mathrm{L}}
$$

where $L$ is the crystal length. An alternative expression to Eqn. (2.73) whose form is 
often encountered in the literature is

$$
\left(\mathrm{FWHM}_{\text {sinc }^{2}} \times \mathrm{L}\right)=\frac{5.56622951}{\beta_{\text {variable }}}
$$

The FWHM of the $\operatorname{sinc}^{2}$ response for various types of de-tuning is very useful to know when trying to establish a crystal's merit for a particular SHG application that requires for instance, a high tolerance to misalignment of the crystal $(\Delta \theta)$ or to temperature change of the crystal $(\Delta T)$.

Eqns. (2.70) - (2.72) can be used to calculate a particular sensitivity if $\Delta \mathrm{k}^{\prime} \mathrm{s}$ mathematical dependence on the particular variable is known. Eqns. (2.70) $-(2.72)$ express the sensitivities to first order as a particular slope of $\Delta \mathrm{k}$. We can therefore determine a particular sensitivity by calculating the derivative of $\Delta \mathrm{k}$ with respect to that variable: Let's discuss this for the spectral sensitivity. We can write $\Delta k$ as a function of wavelength using Eqns. (2.55) and (2.57) - (2.59). We can then calculate the change in $\Delta k$ from zero for a given $\Delta \lambda$ (i.e. for a given change in wavelength from the phasematched wavelength) which we denote as $\Delta(\Delta \mathrm{k}(0))$. We then divide this by the given $\Delta \lambda$ value (i.e. $\frac{\Delta(\Delta k(0))}{\Delta \lambda}$ ) to determine a value for $\beta_{\lambda}$. Similarly, we can calculate $\beta_{\theta}$ from $\Delta k$ when Eqn. (2.41) is used to express the refractive indices appearing in $\Delta \mathrm{k}$ as a function of angle.

The sensitivities can also be determined experimentally by measuring the $2 \omega$ irradiance as $\Delta \mathrm{k}$ is tuned through zero (for instance by rotating the crystal) and then fitting Eqn. (2.52) to the data. 


\subsubsection{Walk-off}

Walk-off [17] as was discussed in section 2.3.2 refers to how the two orthogonal polarizations of a wave propagating through an anisotropic crystal will in general propagate in different directions. For SHG, walk-off occurs between the $1 \omega$ and $2 \omega$ waves when the propagation direction is not along a dielectric axis. This effect will limit the interaction length since the $1 \omega$ wave and the $2 \omega$ wave will separate as they propagate or, in other words, it will limit the maximum crystal length that would be effectively used.

We will not derive the following equations because they are standard results that are commonly used in the field of nonlinear optics. Consider the SHG interaction between two waves. If one wave is polarized along a dielectric direction and the other is not, the two waves will experience walk-off and the propagation directions of the two waves will be at a small angle. This small angle is called the walk-off angle $(\rho)$. The effect of the walk-off angle for SHG interactions can be expressed as a limit to the effective interaction length [18] by

$$
L^{\prime}=\frac{L}{\left(1+\frac{L \rho}{w_{0} \sqrt{\pi}}\right)^{\frac{1}{2}}}
$$

where $L$ is the physical length of the crystal, $\rho$ is the walk-off angle, and $w_{0}$ is the beam waist size. The walk-off angle ( $\rho$ ) for a given propagation direction [11] can be calculated using 


$$
\tan \rho=n^{2}\left[\left(\frac{s_{x}}{n^{-2}-n_{x}^{-2}}\right)^{2}+\left(\frac{s_{y}}{n^{-2}-n_{y}^{-2}}\right)^{2}+\left(\frac{s_{z}}{n^{-2}-n_{z}^{-2}}\right)^{2}\right]^{-\frac{1}{2}}
$$

where $\mathbf{n}$ is the refractive index in the direction of the given wave's polarization, $s_{i}$ are the direction cosines for the propagation vector $\mathbf{s}$, and $n_{i}$ are the principal refractive indices along the dielectric axes. These expressions will be used to correct for walk-off effects in the SHG data collected in Chapter 3.

\subsubsection{Non-critical phasematching (NCPM)}

Phasematched interactions that are not along dielectric axes are called critically phasematched. When nonlinear interactions are phasematched for propagation down a dielectric axis it is called non-critical phasematching (NCPM). NCPM is a very advantageous situation for two reasons. First, the angular sensitivity $\left(\beta_{\theta}\right)$ has a quadratic dependence on the misalignment angle (i.e. $(\Delta \theta)^{2}$ ) rather than a linear dependence as for critical phasematching [6]. The reason for the quadratic dependence on the misalignment angle in the NCPM case is because for small deviations (i.e. $\Delta \theta<1$ ) away from propagation down a dielectric axis, the index ellipsoid looks approximately circular, and therefore $\frac{\mathrm{d} \Delta \mathrm{k}}{\mathrm{d} \theta} \approx 0$ which means to first order the angular sensitivity is zero. The quadratic dependence on the misalignment angle in reality gives rise to a relatively broad $\operatorname{sinc}^{2}$ response as $\Delta \theta$ varies which implies that NCPM interactions have relatively small but finite angular sensitivities $\left(\beta_{\theta}\right)$. A good descriptive illustration for the difference in angular sensitivities between critically phasematched interactions and NCPM interactions is shown in Fig. 2.8. The angular width of the NCPM signal in Fig. 2.8 is much larger 

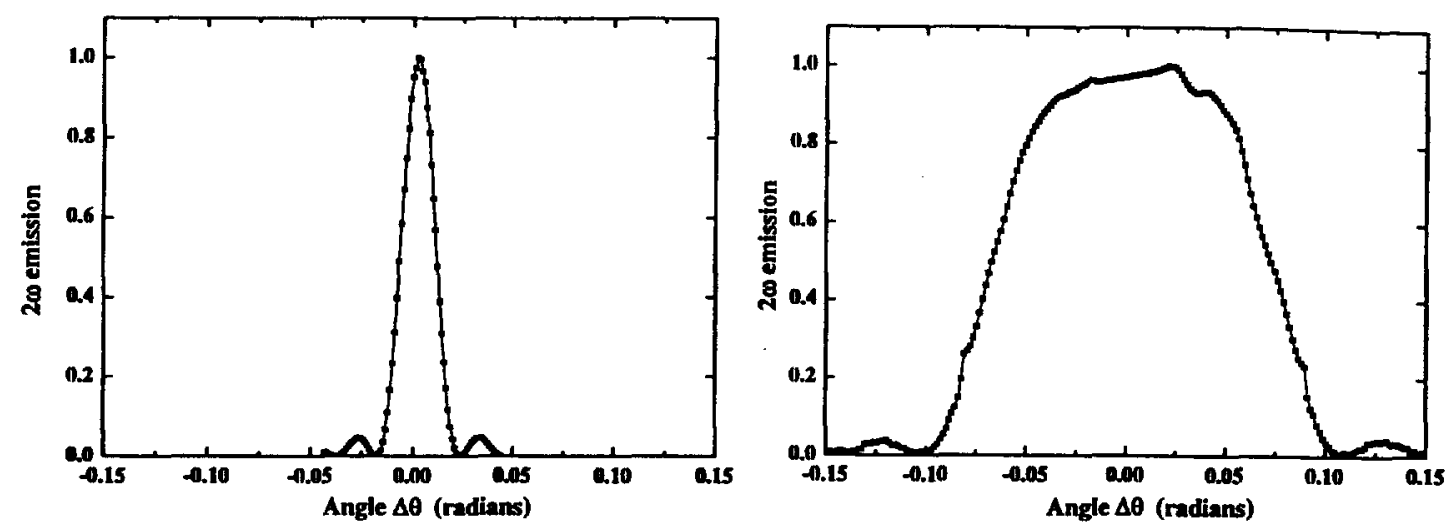

Figure 2.8. Experimental $2 \omega$ irradiance data for equal length crystals illustrating the difference in response for critically phasematched SHG (left) and NCPM SHG (right). The tuning angle $\Delta \theta$ is measured from the phasematching direction (i.e. $\Delta \mathrm{k}=0$ ).

$(-10 x)$ than for the critically phasematched case and since $\beta_{\theta}$ is inversely proportional to the widths in Fig. 2.8 this implies that the NCPM interaction has an -10 times lower angular sensitivity.

A second advantage of NCPM is that there are no walk-off effects due to double refraction. Walk-off is eliminated for propagation down a dielectric axis because the wavevector and Poynting vector point in the same direction as was previously discussed. An immediate benefit is that long crystal lengths can be utilized to increase the SHG efficiency for a given $1 \omega$ irradiance, as opposed to critically phasematched interactions that have interaction lengths (crystal lengths) which are limited to the walk-off length given in Eqn. (2.75). One of the goals of the experimental investigations in Chapters 3 and 5 is to identify crystals that have useful NCPM wavelengths (i.e. $1.047 \mu \mathrm{m}, 1.053$ $\mu \mathrm{m}$, or $1.064 \mu \mathrm{m}$ ). 


\subsection{Optical parametric oscillation}

\subsubsection{Coupled-amplitude equations}

In Chapter 4, experiments will be conducted with the COB crystals to determine their potential for use in an optical parametric oscillator (OPO). In this section, we will discuss the important fundamentals of OPOs relevant to evaluating the experimental data we will present in Chapter 4. An OPO is a resonant optical cavity that contains a nonlinear crystal as the gain medium. Typically, an OPO is pumped by a strong monochromatic wave and through reverse sum frequency generation (which will be referred to as the OPO process from here on) produces two other waves from noise that are known as the signal and idler waves. The pump, signal, and idler waves have

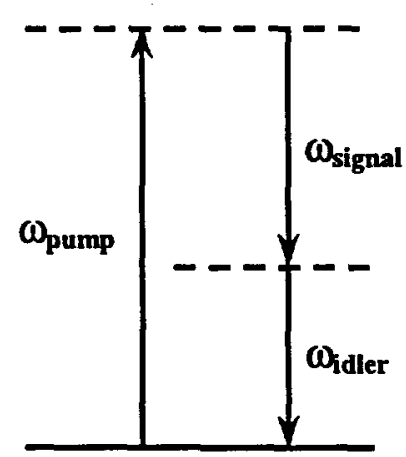

Figure 2.9. Energy level description of the OPO process showing the relation between the pump ( $\left.\omega_{\text {pump }}\right)$, signal $\left(\omega_{\text {signal }}\right)$, and idler frequencies $\left(\omega_{\text {idler }}\right)$ where $\omega_{\text {pump }}=\omega_{\text {signal }}$ $+\omega_{\text {idler }}$.

frequencies that are related by $\omega_{\text {pump }}=\omega_{\text {signal }}+\omega_{\text {idler }}$ (alternatively written $\omega_{3}=\omega_{1}+\omega_{2}$ ) as shown in Fig. 2.9. We will focus our discussion on the singly resonant oscillator (SRO) configuration shown in Fig. 2.10. We first derive equations that describe the amplitudes of the interacting waves in the SRO. Once we have the expressions for the amplitudes we will then derive expressions for the round-trip gain and oscillation 
threshold. We will treat the problem one-dimensionally (i.e. in the $\mathrm{z}$ direction) for simplicity.

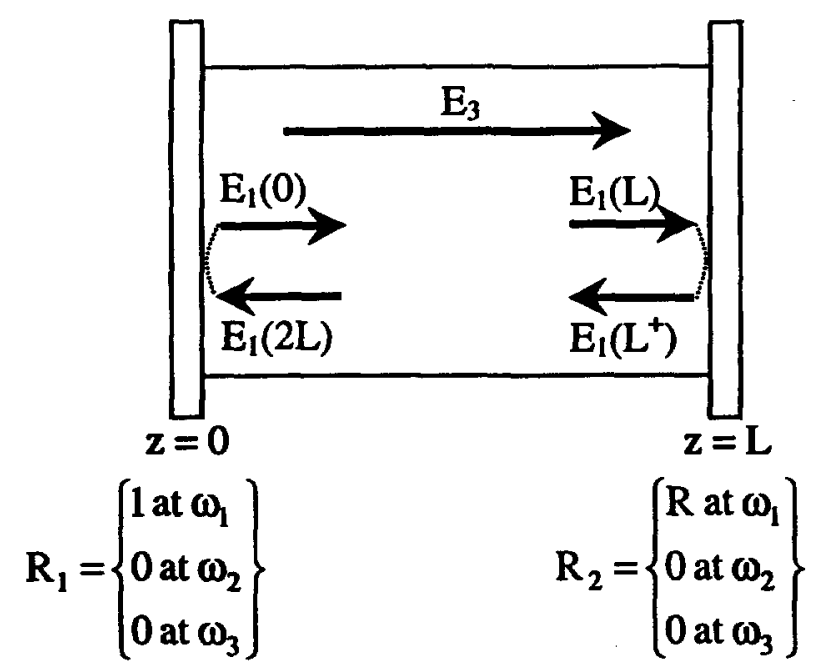

Figure 2.10. Singly resonant optical parametric oscillator (SRO). The resonator cavity is composed of flat mirrors with the space between the mirrors completely filled with a nonlinear crystal. $\mathbf{R}_{\mathbf{1}}$ and $\mathbf{R}_{\mathbf{2}}$ are the resonator mirrors' reflectivities. $\mathbf{E}_{\mathbf{3}}$ is the strong pump wave and $E_{1}$ is the signal wave that is resonated. Note the idler wave $\left(E_{2}\right)$ is not shown.

Coupled equations relating the amplitudes of the 3 interacting waves $E_{1}(z, t)$ (signal), $E_{2}(z, t)$ (idler), and $E_{3}(z, t)$ (pump) (note that here the subscripts refer to the three frequencies) can be derived for SFG by a very similar method as was used to find Eqns. (2.49) and (2.50) for SHG. The details can be found in Ref. [7] and so we will only quote the final results. For the case of no absorption losses and slowly-varying field amplitudes, the fields $E_{1}, E_{2}$, and $E_{3}$ are related by the following set of three coupled equations

$$
\frac{\partial E_{1}}{\partial z}=\frac{8 \pi i \omega_{1}^{2} d_{\text {eff }} E_{3} E_{2}^{*}}{k_{1} c^{2}} e^{i \Delta k z}
$$




$$
\begin{aligned}
& \frac{\partial E_{2}}{\partial z}=\frac{8 \pi i \omega_{2}^{2} d_{e f f} E_{3} E_{1}^{*}}{k_{2} c^{2}} e^{i \Delta k z} \\
& \frac{\partial E_{3}}{\partial z}=\frac{8 \pi i \omega_{3}^{2} d_{e f f} E_{1} E_{2}}{k_{3} c^{2}} e^{-i \Delta k z}
\end{aligned}
$$

where $\Delta k=k_{3}-k_{1}-k_{2}$. We note in passing that these are the basic amplitude equations for parametric amplification.

We seek solutions to Eqns. (2.77) $-(2.79)$ for the conditions that $\Delta k=0$ and no pump depletion, i.e. $\mathrm{E}_{3}$ is constant in z. Applying these two conditions reduces Eqns. (2.77) and (2.78) to

$$
\begin{aligned}
& \frac{\partial E_{1}}{\partial z}=C_{1} E_{2}^{*} \\
& \frac{\partial E_{2}}{\partial z}=C_{2} E_{1}^{*}
\end{aligned}
$$

Differentiation of Eqn. (2.81) and substitution of Eqn. (2.80) gives

$$
\frac{\partial^{2} E_{2}}{\partial z^{2}}=\kappa^{2} E_{2}
$$

where $\kappa^{2}=C_{1}^{*} C_{2}=\frac{64 \pi^{2} d_{e f f}^{2} \omega_{1}^{2} \omega_{2}^{2}}{k_{1} k_{2} c^{4}}\left|E_{3}\right|^{2}$. A general solution to Eqn. (2.82) is given by

$$
\mathrm{E}_{2}(\mathrm{z})=\mathrm{A} \sinh \kappa \mathrm{z}+\mathrm{B} \cosh \kappa \mathrm{z}
$$

Applying the boundary condition that $E_{2}(0)=0$ to Eqn. (2.83) and using Eqn. (2.81) with $E_{1}(0)=$ specified we obtain solutions for $E_{1}(z)$ and $E_{2}(z)$ given by 


$$
\begin{gathered}
E_{1}(z)=E_{1}(0) \cosh \kappa z \\
E_{2}(z)=i\left(\frac{n_{1} \omega_{2}}{n_{2} \omega_{1}}\right)^{\frac{1}{2}} \frac{E_{3}}{\left|E_{3}\right|} E_{1}^{*}(0) \sinh \kappa z
\end{gathered}
$$

These are the solutions for the signal and idler wave amplitudes as a function of $\mathrm{z}$ for the conditions that the pump wave amplitude $\left(E_{3}\right)$ is constant and $\Delta \mathrm{k}=0$. Eqns. (2.84) and (2.85) will allow us to derive expressions in the next section for the round-trip gain and oscillation threshold for the SRO depicted in Fig. 2.10.

\subsubsection{Round-trip gain}

We will calculate the roundtrip gain in terms of the fractional increase in the amplitude of $E_{1}$ for a roundtrip pass around the resonator as shown in Fig. 2.10. Starting from the left, traversing the resonator and reflecting off the right mirror, the increase in $\mathrm{E}_{1}$ can be written using Eqn. (2.84) and a phase change as

$$
E_{1}\left(L^{+}\right)=R E_{1}(0) e^{-i k_{1} L} \cosh K z
$$

where $\mathrm{R}$ is the reflectivity of the right cavity mirror in Fig. 2.10. On the return path from right to left, there is no parametric interaction since the signal and pump waves are traveling in opposite directions, however there is still a phase change and so we write

$$
E_{1}(2 L)=E_{1}\left(L^{+}\right) e^{-i k_{1} L}=R E_{1}(0) e^{-2 i k_{1} L} \cosh \kappa z
$$

Eqn. (2.87) can be used to calculate the fractional roundtrip gain as 


$$
\text { Roundtrip gain } \equiv \frac{E_{1}(2 L)}{E_{1}(0)}=\operatorname{Re}^{-i k_{1} L} \cosh \kappa Z
$$

In general, we require that the phase term in Eqn. (2.88) be equal to an integer multiple of $2 \pi$ in order to have oscillation (i.e. $k_{l} L=2 m \pi$ for $m=$ integer). In other words the oscillation frequency must correspond to a longitudinal mode of the resonator. We will now use Eqn. (2.88) to derive a condition for oscillation threshold in the SRO.

\subsubsection{Oscillation threshold}

Oscillation threshold will be reached when the phase condition is met and the roundtrip gain is equal to one. We can express this mathematically using Eqn. (2.88) as

$$
\operatorname{Rcosh\kappa }_{\mathrm{th}} \mathrm{z}=1
$$

where we have set $-\mathrm{ik}_{1} \mathrm{~L}=2 \pi \mathrm{i}$ in Eqn. (2.88) and $\kappa_{\mathrm{th}}$ is written to specify that this corresponds to the pump amplitude $\left(\mathrm{E}_{3}=\mathrm{E}_{3 \mathrm{th}}\right)$ that satisfies Eqn. (2.89). By assuming $R$ $\approx 1$ and using the first two terms in the power series expansion for cosh, we can solve Eqn. (2.89) for $E_{3 \text { th }}$, which gives

$$
E_{3 t h}=\frac{\sqrt{2} \sqrt{(1-R)}}{L} \frac{c^{2} \sqrt{k_{1} k_{2}}}{8 \pi d_{\text {eff }} \omega_{1} \omega_{2}}
$$

It will be useful in Chapter 4 to have the oscillation threshold expressed in terms of the threshold pump irradiance. In c.g.s. units, irradiance is related to the field amplitude by $\mathrm{I}=\frac{\mathrm{nc}}{2 \pi} \mathrm{EE} \mathrm{E}^{*}$. Therefore we calculate the oscillation threshold pump irradiance for our SRO to be 


$$
I_{3 t h}=\frac{n^{3} c \lambda_{1} \lambda_{2}(1-R)}{256 \pi^{5} d_{e f f}^{2} L^{2}}
$$

This expression is written in c.g.s. units for the case of plane waves, perfect phasematching, and an un-depleted pump wave.

The oscillation threshold given by Eqn. (2.91) is for the most ideal situation possible. However, one not often has the chance to use perfect plane waves in the laboratory and so we would like a threshold estimate that is based on a more physically realistic situation. Brosnan and Byer [19] derive an expression for the threshold of a SRO under more realistic conditions. They consider a pulse pumped SRO where the pump beam has a gaussian profile in space and time and the signal and idler beams are gaussian in space. Their model also accounts for beam walk-off between the pump and generated beams. Eqn. (2.92) is the analytical expression derived by Brosnan and Byer [19] for the threshold pump fluence $\left(F_{t h}\left(J / \mathrm{cm}^{2}\right)\right)$ of a pulse pumped SRO. Eqns. (2.91) and (2.92) will both be used in Chapter 4 to estimate the pump fluence needed to reach oscillation threshold in a NCPM SRO based on YCOB.

$$
F_{\text {th }}=\frac{2.25 \tau}{\operatorname{kg}_{s} L^{2}}\left[\frac{L}{2 \tau c} \ln \left(\frac{P_{n}}{P_{o}}\right)+2 \alpha \ell+\ln \left(\frac{1}{\sqrt{R}}\right)+\ln 2\right]^{2}
$$

where,

$\tau=1 / \mathrm{e}^{2}$ pump pulselength

$\kappa=$ nonlinear coupling constant for $Y C O B=\frac{8 \pi^{2} d_{e f f}^{2}}{\lambda_{s} \lambda_{i} n_{s} n_{i} n_{p} \varepsilon_{o} c}$

$\mathrm{g}_{s}=$ signal beam spatial mode coupling coefficient $=\frac{\mathrm{w}_{\mathrm{p}}^{2}}{\mathrm{w}_{\mathrm{p}}^{2}+\mathrm{w}_{\mathrm{s}}^{2}}=\left(\right.$ for $\mathrm{w}_{\mathrm{p}}=$ pump beam spot size and $w_{s}=$ signal beam spot size) 
$L=$ effective parametric gain length $=$ crystal length

$\mathrm{L}=$ optical cavity length $=\mathrm{L}^{\prime}($ physical cavity length $)+(\mathrm{n}-1) \ell($ physical crystal length)

$\frac{P_{n}}{P_{0}}=\frac{\text { signal threshold power }}{\text { signal noise power }} \equiv 10^{14}$ (determined in general by Brosnan and Byer [19])

$\alpha=$ crystal absorption coefficient at $\lambda_{\mathrm{s}}$

$\ell=$ crystal length

$\mathbf{R}=$ reflectivity of the output coupler

\subsubsection{NCPM and degenerate wavelengths}

Optical parametric oscillators that have nonlinear interactions that are NCPM are very advantageous from a device point of view. NCPM has inherent low angular sensitivity (as discussed in section 2.4.6) which relaxes the alignment criteria on resonator designs and operation. The low angular sensitivity of NCPM also helps the efficiency of an oscillator when it is necessary to strongly focus the pump beam into the cavity.

When the idler and signal beams in an OPO have the same frequency the oscillator is said to be operating in the degenerate mode (i.e. $\omega_{3}=\omega_{1}+\omega_{1}$ ). In this mode the oscillator generates waves with a single frequency or, in other words from each pump photon two signal photons are created. Another way to look at this is that a degenerate OPO will completely convert a strong pump beam into strong single frequency signal beam. In Chapter 4 we will experimentally investigate the potential of utilizing the COB crystals in both a NCPM and degenerate OPO. 


\subsection{Linear electro-optic effect}

\subsubsection{General introduction}

In Chapter 5, experiments will be conducted with YCOB to determine its potential for use in Q-switches [20] and electro-optic modulators [7]. A Q-switch or an electro-optic modulator is a device based on a crystal (typically) that exhibits the electro-optic effect. We will construct electro-optic modulators in Chapter 5 using YCOB crystals and will measure the transmitted irradiance through the modulators as a function of the applied dc or low frequency voltage. These measurements will allow us to determine the (effective) electro-optic coefficients for YCOB. In this chapter, we will discuss the important fundamentals of the electro-optic effect relevant to interpreting the experimental data we will present in Chapter 5 .

The electro-optic effect is the change in principal refractive indices of a crystal induced by the presence of a dc (or low frequency) electric field. We will be interested in the change of refractive index that depends linearly on the strength of the applied electric field. This is known as the linear electro-optic effect or the Pockels effect. This effect was studied extensively by Friedrich Carl Alwin Pockels in 1893. The linear electrooptic effect can be treated as the sum frequency mixing (SFG) of two waves ( $\omega_{1}$ and $\omega_{2}$ ) with frequencies $\omega$ and 0 creating a third wave $\left(\omega_{3}\right)$ at frequency $\omega$ with a different phase. This can be described in terms of a $\chi^{(2)}$ process using Eqn. (2.12) as

$$
\mathrm{P}_{\mathrm{i}}\left(\omega_{3}=\omega\right)=2 \sum_{\mathrm{jk}} \chi_{\mathrm{ijk}}^{(2)}\left(\omega_{3}=\omega+0 ; \omega, 0\right) \mathrm{E}_{\mathrm{j}}(\omega) \mathrm{E}_{\mathrm{k}}(0)
$$

Although the linear electro-optic effect can be described in terms of a second-order 
nonlinear susceptibility, historically a different mathematical approach has been used and it will be this alternative approach used for the derivations in this chapter [5]. In order to interpret the experimental data in Chapter 5, we will need to be able to calculate how the transmitted irradiance through a YCOB modulator depends on the voltage applied to the crystal. We will need to know how the principal refractive indices of a crystal are modified by the application of a dc or low-frequency electric field in order to calculate the transmitted irradiance. We seek a relation that relates the change in refractive indices $\left(\delta n_{i j}\right)$ to the components of the applied electric field $\left(E_{k}\right)$ of the following general form

$$
\delta \mathrm{n}_{\mathrm{ij}} \approx \sum_{\mathrm{k}} \mathrm{r}_{\mathrm{ijk}} \mathrm{E}_{\mathrm{k}} \equiv \Delta_{\mathrm{ij}}
$$

where $r_{\mathrm{ijk}}$ is the electro-optic tensor [5]. Before proceeding with deriving this relation for the changes in the refractive indices, we will discuss some of the underlying basics of the linear electro-optic effect including the $r_{i j k}$ tensor.

\subsection{2 $r_{i j k}$ coefficients}

In this section we develop a mathematical formalism that describes the linear electrooptic effect. We wish to determine how the index ellipsoid of a crystal is modified when the crystal is subjected to a dc or low-frequency electric field. The modification due to the applied electric field can be conveniently described in terms of the impermeability tensor, $\eta_{i j}[5]$. The impermeability tensor $\left(\eta_{i j}\right)$ is defined by

$$
E_{i}=\sum_{j} \eta_{i j} D_{j}
$$

Note that this relation is the inverse of Eqn. (2.27) and hence, the impermeability tensor 
$\left(\eta_{i j}\right)$ is the inverse of the dielectric tensor $\left(\varepsilon_{i j}\right)$. (Since $\varepsilon_{i j}$ is symmetric (Eqn. (2.35)) and $\eta_{i j}$ is the inverse of $\varepsilon_{\mathrm{ij}}$, then $\eta_{\mathrm{ij}}$ must also be symmetric.) Preceding as with Eqn. (2.30), we write the electric energy density $\left(W_{e}\right)$ as

$$
\mathrm{W}_{\mathrm{e}}=\frac{1}{8 \pi}(\overrightarrow{\mathrm{E}} \cdot \overrightarrow{\mathrm{D}})=\frac{1}{8 \pi} \sum_{\mathrm{ij}} \eta_{i j} \mathrm{D}_{\mathrm{i}} \mathrm{D}_{\mathrm{j}}
$$

Writing out the far right-hand side of this expression and using $x=D_{x} / \sqrt{8 \pi W_{e}}, y=$ $D_{y} / \sqrt{8 \pi W_{e}}$, and $z=D_{z} / \sqrt{8 \pi W_{e}}$ we obtain

$$
\eta_{x x} x^{2}+\eta_{y y} y^{2}+\eta_{z z} z^{2}+2 \eta_{y z} y z+2 \eta_{x y} x z+2 \eta_{x y} x y=1
$$

This represents the equation for the surface of an ellipsoid. Comparing Eqn. (2.97) to Eqns. (2.36) and (2.38), we see that if $x, y$, and $z$ are chose along the crystal's dielectric directions, then Eqn. (2.97) reduces to

$$
\eta_{x x} x^{2}+\eta_{y y} y^{2}+\eta_{z z} z^{2}=1
$$

Comparing Eqn. (2.98) to Eqn. (2.40), we find that the following relations for the $\eta_{\mathrm{ii}}$ coefficients must be true

$$
\eta_{\mathrm{xx}}=\frac{1}{\mathrm{n}_{\mathrm{x}}^{2}} \quad \eta_{\mathrm{yy}}=\frac{1}{\mathrm{n}_{\mathrm{y}}^{2}} \quad \eta_{\mathrm{zz}}=\frac{1}{\mathrm{n}_{\mathrm{z}}^{2}}
$$

where $n_{x}, n_{y}$, and $n_{z}$ are the principal refractive indices of the index ellipsoid.

We next assume that the impermeability tensor can be written as a power series [5] in the components of the applied dc electric field $\left(\mathrm{E}_{\mathrm{k}}\right)$ as 


$$
\eta_{i j}=\eta_{i j}^{(0)}+\sum_{k} r_{i j k} E_{k}+\cdots=\eta_{i j}^{(0)}+\Delta_{i j}+\cdots
$$

here $\mathbf{r}_{\mathrm{ijk}}$ is the linear electro-optic tensor and it describes the first-order modification to the impermeability tensor due to an applied dc electric field. The $r_{i j k}$ tensor is a third-rank tensor and its components will be referred to as the linear electro-optic coefficients [21]. Since the tensor $\eta_{i j}$ is symmetric (i.e. $\left.\eta_{i j}=\eta_{j i}\right)$ then the linear electro-optic tensor $\left(r_{i j k}\right)$ must also be symmetric in its first two indices. Therefore it will be convenient to write

$r_{i j k}$ in contracted notation as $r_{h k}$ according to the following conventions for the indices:

$\begin{array}{ccccccc}\text { ij: } & 11 & 22 & 33 & 23,32 & 31,13 & 12,21 \\ \text { h: } & 1 & 2 & 3 & 4 & 5 & 6\end{array}$

We can express $r_{\text {hik }}$ in terms of a $3 \times 6$ matrix as

$$
r_{h k}=\left[\begin{array}{lll}
r_{11} & r_{12} & r_{13} \\
r_{21} & r_{22} & r_{23} \\
r_{31} & r_{32} & r_{33} \\
r_{41} & r_{42} & r_{43} \\
r_{51} & r_{52} & r_{53} \\
r_{61} & r_{62} & r_{63}
\end{array}\right]
$$

From Eqn. (2.100), the first-order modification $\left(\Delta_{\mathrm{ij}}\right)$ to the coefficients of the impermeability tensor $\left(\eta_{i j}\right)$ due to the applied electric field can be written using the contracted notation as

$$
\Delta_{\mathrm{h}}=\sum_{\mathbf{k}} \mathrm{r}_{\mathrm{hk}} \mathrm{E}_{\mathbf{k}}
$$


Eqn. (2.102) allows us to write Eqn. (2.103) in matrix form as

$$
\left[\begin{array}{c}
\Delta_{1} \\
\Delta_{2} \\
\Delta_{3} \\
\Delta_{4} \\
\Delta_{5} \\
\Delta_{6}
\end{array}\right]=\left[\begin{array}{lll}
r_{11} & r_{12} & r_{13} \\
r_{21} & r_{22} & r_{23} \\
r_{31} & r_{32} & r_{33} \\
r_{41} & r_{42} & r_{43} \\
r_{51} & r_{52} & r_{53} \\
r_{61} & r_{62} & r_{63}
\end{array}\right]\left[\begin{array}{l}
E_{x} \\
E_{y} \\
E_{z}
\end{array}\right]
$$

where $E_{\mathrm{i}}$ are the components of the dc or low frequency applied electric field and the quantities $r_{h k}$ are the linear electro-optic coefficients. The quantities $\Delta_{h}$ will be shown in the next section to be directly related to changes in the principal refractive indices of the crystal and can be calculated exactly for a given crystal symmetry with specific orientations of the input polarization, the applied electric field, and the direction of propagation.

The exact form of the electro-optic tensor (Eqn. (2.102)) is constrained by the symmetry properties of the electro-optic crystal. The $\mathbf{r}_{\mathbf{h k}}$ matrices have been previously determined for all the symmetry groups $[5,7,13]$. As we have discussed, YCOB is a 'biaxial crystal and belongs to the monoclinic crystal class with point group $\mathrm{m}$ symmetry with the convention that $m \perp b$. Therefore the $r_{h k}$ tensor (matrix) has the form given in Eqn. (2.105) where some of its components are zero.

$$
r_{\text {hk }} \text { (monoclinic crystal class, point group } m \text { ) }=\left[\begin{array}{ccc}
r_{11} & 0 & r_{13} \\
r_{21} & 0 & r_{23} \\
r_{31} & 0 & r_{33} \\
0 & r_{42} & 0 \\
r_{51} & 0 & r_{53} \\
0 & r_{62} & 0
\end{array}\right]
$$


Eqn. (2.105) is the form for the linear electro-optic tensor that will be used to describe the linear electro-optic effect in the YCOB crystals. The mathematical expressions for the changes of the refractive indices in the YCOB crystals in terms of the coefficients of $\mathrm{r}_{\mathrm{hk}}($ Eqn. (2.105)) and the applied electric field will be derived in the next section.

\subsubsection{Induced birefringence and phase retardation}

A basic description of how the electro-optic effect can be observed is given in Fig. 2.11. For this general discussion, we are assuming that the crystal has no natural birefringence. However, when the voltage is applied to the crystal, by definition of the electro-optic effect, a birefringence is induced [13] and we will assume the induced principal dielectric axes are along the $y$ and $z$ directions as shown in Fig. 2.11. Therefore, in Fig. 2.11 if the input beam's polarization $\left(\vec{E}_{\text {inc }}\right)$ is assumed to be at $45^{\circ}$ to the z-axis, then with the voltage applied the polarization of the output beam ( $\left.\overrightarrow{\mathrm{E}}_{\text {out }}\right)$ will

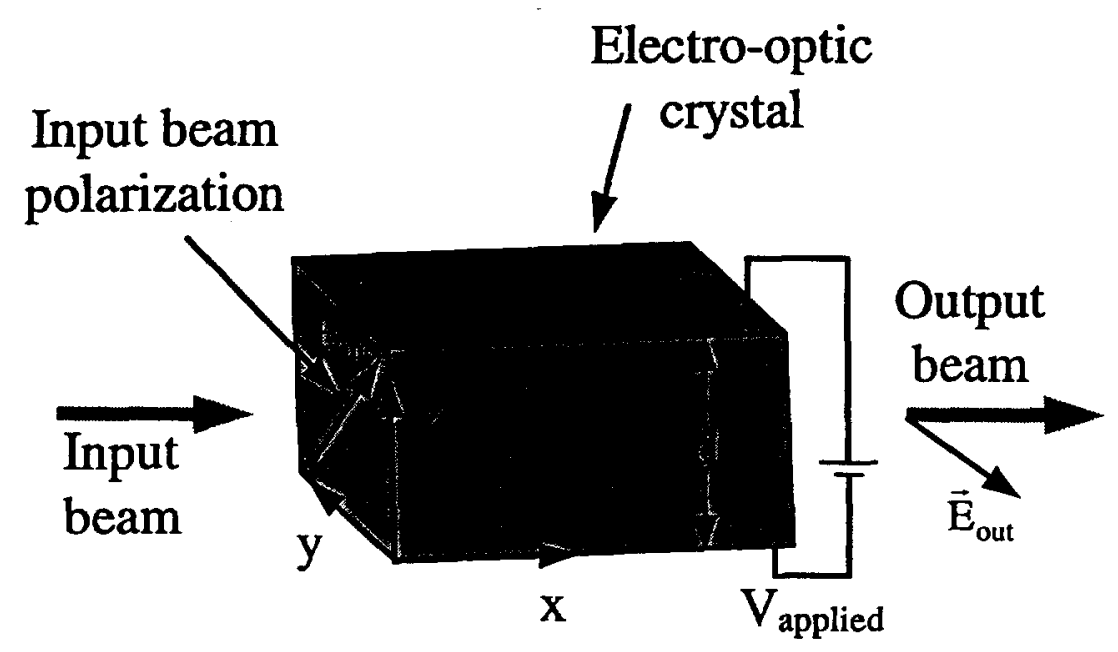

Figure 2.11. Illustration of the electro-optic effect. $\overrightarrow{\mathrm{E}}_{\text {inc }}$ is at $45^{\circ}$ to the $\mathrm{z}$ direction. The voltage $\left(V_{\text {applied }}\right)$ applied to the crystal induces a birefringence and causes a rotation of the input beam's polarization as shown by $\overrightarrow{\mathrm{E}}_{\text {out }}$. 
be rotated relative to the input beam $\left(\overrightarrow{\mathrm{E}}_{\mathrm{inc}}\right)$ as shown. This rotation occurs because as the two orthogonal polarizations of the beam $\left(E_{y}\right.$ and $\left.E_{z}\right)$ traverse the crystal the birefringence induced by the applied voltage imparts a phase retardation between the two polarizations [13]. We will choose to label this phase retardation as $\Gamma$ (In the following, whenever we speak of a phase retardation and label it by $\Gamma$ we implicitly mean the net phase retardation between the waves). Therefore if the input beam in Fig. 2.11 is given by

$$
\overrightarrow{\mathrm{E}}_{\mathrm{inc}}=\frac{1}{\sqrt{2}}(-\mathrm{E} \hat{\mathrm{y}}+\mathrm{E} \hat{\mathrm{z}}) \mathrm{e}^{\mathrm{i} \omega \mathrm{t}}
$$

then with the voltage applied the output beam is given by

$$
\overrightarrow{\mathrm{E}}_{\text {out }}=\frac{\mathrm{e}^{-i \beta}}{\sqrt{2}}\left(-\mathrm{E} \hat{y}+E e^{-i \Gamma} \hat{z}\right) e^{i \omega t}
$$

where $\beta$ is a common phase factor between the $y$ and $z$ components of $\overrightarrow{\mathrm{E}}_{\text {out }}$. We are assuming here that there are no reflections and that the crystal is totally transmitting. The polarization direction of the field described in Eqn. (2.107) is given by $\left(-\mathrm{E} \hat{y}+\mathrm{Ee}^{-\mathrm{i} \Gamma} \hat{z}\right)$ which can be seen to point in a different direction than that given in Eqn. (2.106). If a polarizer is placed in the output beam in Fig. 2.11, the transmitted irradiance will change when the voltage is applied. By measuring the change in transmitted irradiance through the polarizer, we can determine the material's electro-optic coefficients $\left(r_{i j k}\right)$.

The exact mathematical expression for the phase retardation $(\Gamma)$ depends on the particular crystal symmetry of the electro-optic crystal, the orientation of the input 
polarization, the direction of the applied electric field ( $\left.\mathrm{V}_{\text {applied }}\right)$, and the direction of the beam propagation relative to the crystal's dielectric directions. We would like to point out that since $\mathrm{YCOB}$ is a biaxial crystal there will be a static contribution to the phase retardation due to the natural birefringence of the crystal in addition to the phase retardation induced by the applied voltage. Fig. 2.11 shows a transverse arrangement (i.e. the applied voltage is transverse to the propagation direction of the beam), however it is also possible to have a longitudinal arrangement where the applied voltage is parallel to the direction of propagation. In Chapter 5, we will conduct experiments to measure the (effective) linear electro-optic coefficients for YCOB using both transverse and longitudinal arrangements. The exact expressions for the phase retardation $(\Gamma)$ produced by $\mathrm{YCOB}$ in the transverse and longitudinal arrangements used in our experiments are derived in the next three sections.

\subsubsection{YCOB phase retardation}

We are ultimately trying to relate the transmitted irradiance through a YCOB electrooptic modulator to the voltage applied to the crystal. In general, an electro-optic modulator is a device that is composed of a polarizer followed by an electro-optic crystal followed by a second polarizer (analyzer). The first polarizer ensures that the input beam has a specific input polarization that is typically chosen perpendicular to the analyzer.

A specific example of the type of modulator we will consider is the transverse configuration shown in Fig. 2.12. The quarter-wave plate ensures that circular polarization is incident on the crystal, i.e. equal $E_{y}$ and $E_{z}$ components. If we can determine the output (transmitted) polarization components or, in other words, the electric field components after the beam traverses the modulator then we can calculate 
the transmitted irradiance. We need to know the effect that each individual optical element in the modulator has on the polarization of the beam in order to determine the final output polarization. Therefore, we need to calculate the net phase retardation $(\Gamma)$ imparted between the two orthogonal polarizations of the beam by the different optical elements in Fig. 2.12. The Jones calculus [7,22] will be used to make this calculation.

The Jones calculus was developed in 1941 by R. C. Jones [22] and is a matrix method that can be used to calculate the effect a series of optical elements will have on the polarization state of a beam. The polarization state of the beam is represented by a two component vector ( $2 \times 1$ matrix) while each optical element is represented by a $2 \times 2$ matrix. The basic physical assumption for the Jones calculus to be applicable is that each optical element performs only a linear transformation on the components of the polarization. In the Jones calculus, a matrix is calculated that represents the effect each optical element has on the polarization state of the beam. The matrices for all the optical elements are then multiplied in the order that the elements are encountered (i.e. in our case as the modulator is traversed). The input polarization vector is then multiplied by the result of the matrix multiplication to determine the output polarization vector.

In the remainder of this section, we will derive the Jones matrix that characterizes the net retardation $(\Gamma)$ produced by the YCOB crystal shown in Fig. 2.12. This will illustrate the physical principles of the Jones matrix method as well as relating $\Gamma$ for the crystal to the applied voltage. We will begin by discussing the propagation of light in a birefringent crystal plate. In general, a Jones matrix describes how a certain thickness of a birefringent crystal will add a net phase difference to the two orthogonal polarizations propagating through the crystal. When light propagates in a birefringent crystal, it can be 
represented by a linear superposition of two eigenwaves [8]. The directions of the polarizations for these eigenwaves are orthogonal and will lie along the axes of a crosssection of the index ellipsoid as discussed in section 2.3.2. (In this, and the following derivations in this chapter, it will be assumed that there are no reflections and the optical elements are totally transmitting.)

Let's consider a birefringent crystal plate with its dielectric axes oriented along $x^{\prime}$ and $y^{\prime}$ (where $n_{x^{\prime}} \neq n_{y^{\prime}}$ ) as in Fig. 2.13. Also imagine that a beam is impinging on the plate with its polarization components oriented along the $x$ and $y$ directions. We can express the input polarization in terms of a 2-component Jones vector ( $\mathrm{V}$ ) by

$$
V=\left(\begin{array}{l}
V_{x} \\
V_{y}
\end{array}\right)
$$

where $V_{x}$ and $V_{y}$ are two complex numbers. $V$ should be viewed as the components of the input electric field. To determine how much net phase difference the two components $V_{x}$ and $V_{y}$ acquire in traversing the plate, we first transform $V$ into the prime frame using a coordinate transformation given by

$$
\left(\begin{array}{c}
\mathrm{V}_{\mathrm{x}^{\prime}} \\
\mathrm{V}_{\mathrm{y}^{\prime}}
\end{array}\right)=\left(\begin{array}{cc}
\cos \phi & \sin \phi \\
-\sin \phi & \cos \phi
\end{array}\right)\left(\begin{array}{c}
\mathrm{V}_{\mathrm{x}} \\
\mathrm{V}_{\mathrm{y}}
\end{array}\right) \equiv \mathrm{R}(\phi)\left(\begin{array}{c}
\mathrm{V}_{\mathrm{x}} \\
\mathrm{V}_{\mathrm{y}}
\end{array}\right)
$$

We transform into the prime frame because this is the frame in which the eigenpolarizations will propagate and will acquire their net phase difference [7]. The two waves $V_{x^{\prime}}$ and $V_{y^{\prime}}$ will see different refractive index values $\left(n_{x^{\prime}}\right.$ and $\left.n_{y^{\prime}}\right)$ and will therefore travel through the plate with different speeds. Because of the difference in 


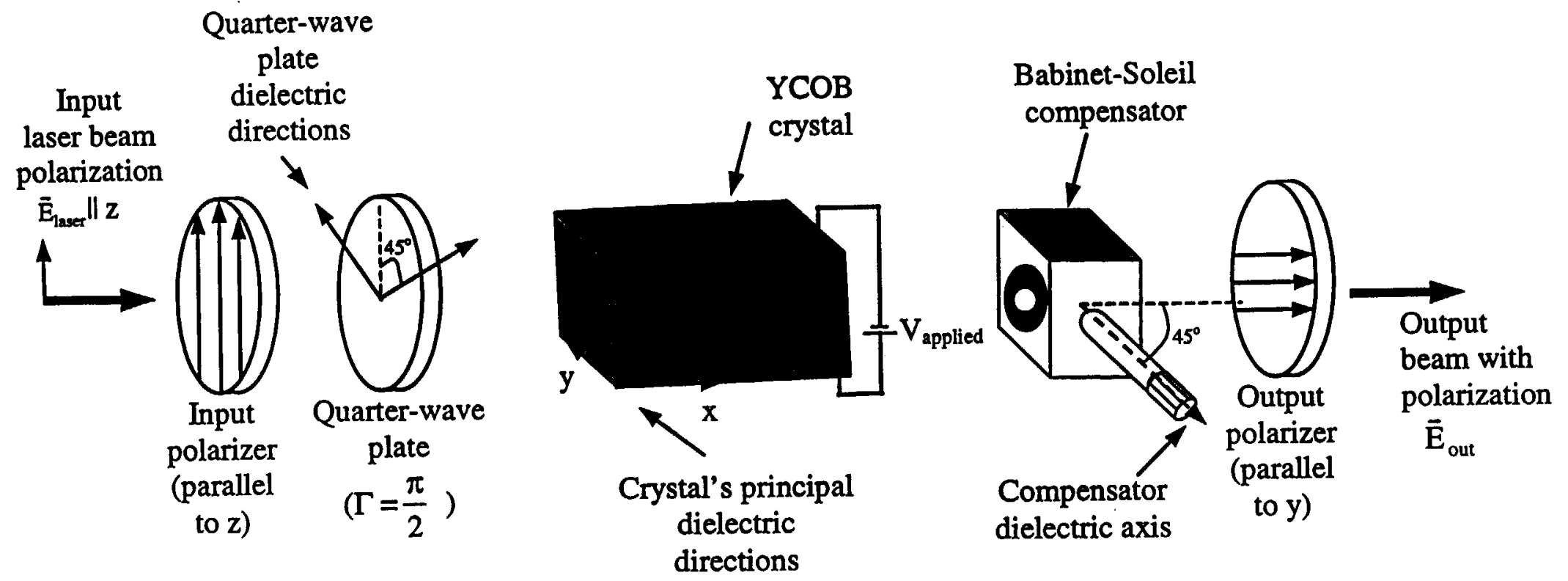

Figure 2.12. A transverse electro-optic YCOB modulator. In this case, the beam propagates along the crystal's $\mathrm{x}$ dielectric axis and the applied voltage is along the $\mathrm{z}$ dielectric axis. 


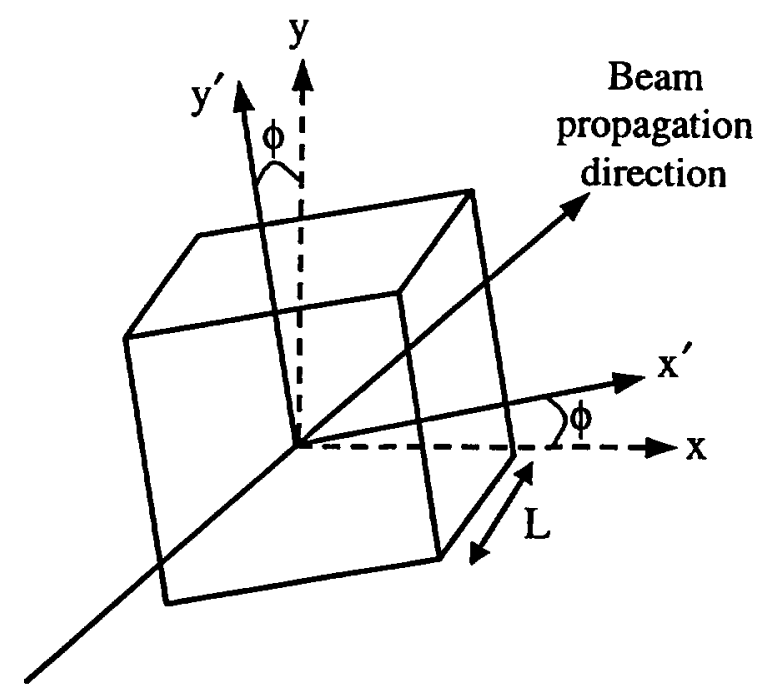

Figure 2.13. A birefringent crystal plate with its dielectric axes ( $x^{\prime}$ and $y^{\prime}$ ) oriented as shown [7].

speeds one component is delayed relative to the other. This delay rotates the polarization of the emerging beam. We now calculate the polarization components of the emerging beam. Let $n_{x^{\prime}}$ and $n_{y^{\prime}}$ be the refractive indices of the plate in the $x^{\prime}$ and $y^{\prime}$ directions in Fig. 2.13. The polarization components of the incident beam in the prime frame $\left(V_{x^{\prime}}\right.$ and $\left.V_{y^{\prime}}\right)$ are related to the polarization components of the exiting beam in the prime frame $\left(V_{x^{\prime}}^{\prime}\right.$ and $\left.V_{y^{\prime}}^{\prime}\right)$ by

$$
\left(\begin{array}{l}
V_{x^{\prime}}^{\prime} \\
V_{y^{\prime}}^{\prime}
\end{array}\right)=\left(\begin{array}{cc}
e^{-\frac{i 2 \pi n_{x^{\prime}} L}{\lambda}} & 0 \\
0 & e^{-\frac{i 2 \pi n_{y^{\prime}} L}{\lambda}}
\end{array}\right)\left(\begin{array}{l}
V_{x^{\prime}} \\
V_{y^{\prime}}
\end{array}\right)
$$

where $L$ is the plate thickness and $\lambda$ is the wavelength of the light beam [7]. The net phase delay or retardation $(\Gamma)$ for the beam is found by taking the difference between the exponents in Eqn. (2.110) because the exponents represent the phase values and we want to know the value of the net phase difference between the waves. Therefore the net phase 
difference (retardation) is equal to

$$
\Gamma=\left(\mathrm{n}_{x^{\prime}}-\mathrm{n}_{y^{\prime}}\right) \frac{2 \pi \mathrm{L}}{\lambda}
$$

Eqn. (2.111) gives a general prescription for calculating the retardation of a briefringent plate (or optical element) if the indices $n_{x^{\prime}}$ and $n_{y^{\prime}}$ are known. We want to write Eqn. (2.110) in terms of $\Gamma$ and so we will introduce a common phase factor for the $x^{\prime}$ and $y^{\prime}$ components of the polarization given by

$$
\beta=\frac{1}{2}\left(n_{x^{\prime}}+n_{y^{\prime}}\right) \frac{2 \pi L}{\lambda}
$$

We can express Eqn. (2.110) in terms of $\Gamma$ and $\beta$ as

$$
\left(\begin{array}{l}
V_{x^{\prime}}^{\prime} \\
V_{y^{\prime}}^{\prime}
\end{array}\right)=e^{-i \beta}\left(\begin{array}{cc}
e^{\frac{i \Gamma}{2}} & 0 \\
0 & e^{\frac{i \Gamma}{2}}
\end{array}\right)\left(\begin{array}{l}
V_{x^{\prime}} \\
V_{y^{\prime}}
\end{array}\right)
$$

We will ignore interference effects and therefore we can neglect the phase factor $\mathrm{e}^{-\mathrm{i} \beta}$. The Jones vector for the polarization state of the emerging beam in the un-prime frame is then found by transforming back to $\mathrm{x}$ and $\mathrm{y}$ using

$$
\left(\begin{array}{c}
V_{x}^{\prime} \\
V_{y}^{\prime}
\end{array}\right)=\left(\begin{array}{cc}
\cos \phi & -\sin \phi \\
\sin \phi & \cos \phi
\end{array}\right)\left(\begin{array}{c}
V_{x^{\prime}}^{\prime} \\
V_{y^{\prime}}^{\prime}
\end{array}\right)
$$

We transform back to $\mathrm{x}$ and $\mathrm{y}$ since in a typical situation we would use a polarizer aligned along either $x$ or $y$ to analyze the polarization state of the beam (as in Fig. 2.12). Combining Eqns. (2.109), (2.113), and (2.114) we can write the transformation for the 
birefringent crystal plate as

$$
\left(\begin{array}{c}
V_{x}^{\prime} \\
V_{y}^{\prime}
\end{array}\right)=R(-\phi) W_{0} R(\phi)\left(\begin{array}{c}
V_{x}^{\prime} \\
V_{y}^{\prime}
\end{array}\right) \equiv W\left(\begin{array}{c}
V_{x}^{\prime} \\
V_{y}^{\prime}
\end{array}\right)
$$

where $\mathrm{W}$ is referred to as the Jones matrix for the plate which is defined by

$$
\mathrm{W} \equiv \mathrm{R}(-\phi) \mathrm{W}_{\mathbf{0}} \mathrm{R}(\phi)
$$

where $R(\phi)$ and $W_{0}$ are given by

and

$$
R(\phi)=\left(\begin{array}{cc}
\cos \phi & \sin \phi \\
-\sin \phi & \cos \phi
\end{array}\right)
$$

$$
W_{0}=e^{-i \beta}\left(\begin{array}{cc}
e^{-\frac{i \Gamma}{2}} & 0 \\
0 & e^{\frac{i \Gamma}{2}}
\end{array}\right)
$$

Eqn. (2.115) can be used to calculate the Jones matrix for the YCOB crystal in Fig. 2.12.

We will now use Eqn. (2.111) to find an expression for the phase retardation due to the YCOB crystal $\left(\Gamma_{\mathrm{xtal}}\right)$ in Fig. 2.12 that explicitly depends on the voltage applied to the crystal $\left(V_{\text {applied }}\right)$. We see from Eqn. (2.111) that $\Gamma$ is related to the difference in refractive indices in the directions of the two orthogonal polarizations. There are two effects that we will need to account for in applying Eqn. (2.111) to the YCOB crystal. First, there is the difference in $n_{y^{\prime}}$ and $n_{z^{\prime}}$ due to the natural (static) birefringence of the YCOB crystal and second, there is the modification of these indices due to the applied voltage through the linear electro-optic effect.

We can use Eqn. (2.97) to calculate the values for the refractive indices (static 
birefringence + electro-optic modification) for the YCOB crystal in Fig. 2.12. Using Eqns. (2.99), (2.100), (2.103), and (2.105) to write out Eqn. (2.97) for the YCOB crystal with the applied electric field $\left(V_{\text {applied }}\right)$ in the direction shown in Fig. 2.12 we obtain

$$
\left(\frac{1}{n_{x}{ }^{2}}+r_{13} E_{z}\right) x^{2}+\left(\frac{1}{n_{y}{ }^{2}}+r_{23} E_{z}\right) y^{2}+\left(\frac{1}{n_{z}{ }^{2}}+r_{33} E_{z}\right) z^{2}+2 r_{53} E_{z} x z=1
$$

where $n_{x}, n_{y}$, and $n_{z}$ are the principal refractive indices of the YCOB crystal. Eqn. (2.119) tells us how the electro-optic effect modifies $n_{x}, n_{y}$, and $n_{z}$ in this case. Let's simplify the terms in Eqn. (2.119) to reveal the explicit change in the indices in the form of $n=n_{0}+\Delta n$ (i.e. $\Delta_{h}$ in Eqn. (2.103)). If we identify the first coefficient of Eqn. (2.119) as

$$
\left(\frac{1}{n_{x}^{\prime 2}}\right) \equiv\left(\frac{1}{n_{x}^{2}}+r_{13} E_{z}\right)
$$

$n_{x}^{\prime \prime}$ can be written as

$$
n_{x}^{\prime \prime}=n_{x}\left(1+n_{x}^{2} r_{13} E_{z}\right)^{-\frac{1}{2}}
$$

We expect that $n_{x}^{2} r_{13} E \ll 1[13]$, and so using $(1+x)^{n} \cong 1+n x$ for small. $x$, Eqn. (2.121) becomes

$$
n_{x}^{\prime \prime}=n_{x}\left(1-\frac{1}{2} n_{x}^{2} r_{13} E_{z}\right)
$$

Now similarly for $n_{y}^{\prime \prime}$ and $n_{z}^{*}$ we have

$$
\begin{aligned}
& n_{y}^{\prime \prime}=n_{y}\left(1-\frac{1}{2} n_{y}^{2} r_{23} E_{z}\right) \\
& n_{z}^{\prime \prime}=n_{z}\left(1-\frac{1}{2} n_{z}^{2} r_{33} E_{z}\right)
\end{aligned}
$$


Using Eqns. (2.122) - (2.124) we can write Eqn. (2.119) as

$$
\frac{x^{2}}{n_{x}^{\prime 2}}+\frac{y^{2}}{n_{y}^{\prime 2}}+\frac{z^{2}}{n_{z}^{\prime 2}}+2 r_{53} E_{z} x z=1
$$

We would like to now transform to a new coordinate system in which Eqn. (2.125) contains no mixed terms, i.e. the system in which it is "diagonalized". This new coordinate system will define the principal axes of the modified index ellipsoid. We can use the coordinate transformation given by [7]

$$
\begin{aligned}
& x=x^{\prime} \cos \theta-z^{\prime} \sin \theta \\
& z=x^{\prime} \sin \theta+z^{\prime} \cos \theta
\end{aligned}
$$

to diagonalize Eqn. (2.125). Performing this transformation on Eqn. (2.125) yields

$$
\left(\frac{1}{n_{x}^{\prime \prime 2}}+r_{53} E_{z} \tan \theta\right) x^{\prime 2}+\frac{y^{2}}{n_{y}^{\prime \prime 2}}+\left(\frac{1}{n_{z}^{\prime 2}}+r_{53} E_{z} \tan \theta\right) z^{\prime 2}=1
$$

where in order for the $\mathrm{xz}$ term to vanish we require that $\tan 2 \theta$ be equal to

$$
\tan 2 \theta=\frac{2 r_{53} E_{z}}{\frac{1}{n_{x}^{\prime 2}}-\frac{1}{n_{z}^{\prime 2}}}
$$

We would like to note that for $E_{z} \approx 10^{6} \mathrm{~V} / \mathrm{m}$ and $r \approx 10 \mathrm{pm} / \mathrm{V}$, as for KDP, that $\theta \approx 0.05^{\circ}$ and so the $\tan \theta$ term can be neglected in Eqn. (2.127). Therefore the expressions for $n_{x}^{\prime \prime}$, $n_{y}^{\prime \prime}$, and $n_{z}^{\prime \prime}$ given in Eqns. (2.122) $-(2.124)$ are the values for the refractive indices of the YCOB crystal which includes the modifications due to the electro-optic effect. 
We can now use Eqn. (2.111) to calculate the net phase retardation due to the YCOB crystal $\left(\Gamma_{\text {xtal }}\right)$ in Fig. 2.12 as

$$
\Gamma_{x t a l}=\left(n_{z}^{\prime \prime}-n_{y}^{\prime \prime}\right) \frac{2 \pi L}{\lambda}
$$

Inserting Eqns. (2.123) and (2.124) gives

$$
\Gamma_{x \text { tal }}=\frac{2 \pi L}{\lambda}\left(\left(n_{z}-n_{y}\right)-\frac{\left(r_{33} n_{z}^{3}-r_{23} n_{y}^{3}\right) E_{z}}{2}\right)
$$

We will now write Eqn. (2.130) in a very useful form using $V_{\text {applied }}=E_{z} d$ as

$$
\Gamma_{\mathrm{xtal}}=\frac{2 \pi L}{\lambda}\left(\left(\mathrm{n}_{\mathrm{z}}-\mathrm{n}_{\mathrm{y}}\right)-\frac{\left(\mathrm{r}_{33} \mathrm{n}_{\mathrm{z}}^{3}-\mathrm{r}_{23} \mathrm{n}_{\mathrm{y}}^{3}\right) \mathrm{V}_{\text {applied }}}{2 \mathrm{~d}}\right)
$$

Eqn. (2.131) tells us the net phase retardation imparted on the beam by the YCOB crystal in Fig. 2.12 as a function of the applied voltage. It is useful and convenient to note two things about Eqn. (2.131).

First, it is customary to refer to the term involving the electro-optic coefficients and the indices-cubed as the effective electro-optic coefficient, $r_{\text {eff }}$. Therefore in this case $r_{\text {eff }}$ is given by

$$
r_{\text {eff }}=\left(r_{33} n_{z}^{3}-r_{23} n_{y}{ }^{3}\right)
$$

Secondly, a quantity that will be very useful later is the value of the applied voltage that makes the second term on the right-hand side of Eqn. (2.131) equal to $\pi$ (i.e. $V_{\pi}$ ). Setting the second term on the right-hand side of Eqn. (2.131) equal to $\pi$ we have 


$$
\pi=\frac{\pi L\left(r_{33} n_{z}^{3}-r_{23} n_{y}^{3}\right) V_{\pi}}{\lambda d}
$$

so solving for $V_{\pi}$ gives

$$
V_{\pi}=\frac{d \lambda}{L\left(r_{33} n_{z}^{3}-r_{23} n_{y}^{3}\right)}=\frac{d \lambda}{L r_{e f f}}
$$

This is called the "half-wave voltage" [7] and is the voltage that will rotate the polarization of the beam by $\frac{\pi}{2}$. It is customary to speak of $V_{\pi}$ for configurations where $d$ $=\mathrm{L}$, therefore in this case $\mathrm{V}_{\pi}$ is given by

$$
\mathrm{v}_{\pi}=\frac{\lambda}{\mathrm{r}_{\mathrm{eff}}}
$$

\subsubsection{Transverse YCOB modulators}

Now that we have $\Gamma_{x t a l}$ for the YCOB crystal in Fig. 2.12 we can calculate the Jones matrix for the crystal. Eqns. $(2.116)-(2.118)$ with $\phi=45^{\circ}$ gives the Jones matrix $\left(W_{x t a l}\right)$ for the YCOB crystal as

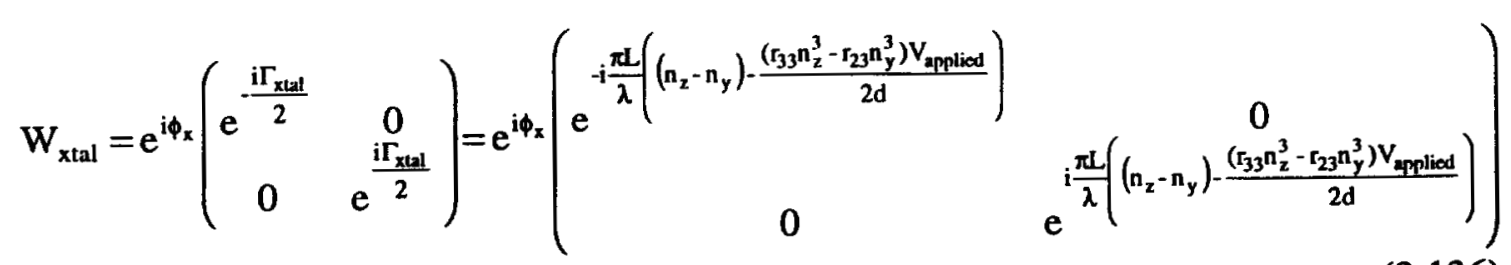

The Jones matrices for the rest of the optical elements shown in Fig. 2.12 will be given without derivation for the sake of brevity but are readily derived using Eqns. 
(2.116) - (2.118) [7]. Starting from left to right in Fig. 2.12 we have for the input polarizer

$$
\mathrm{W}_{\text {input polarizer }}=\left(\begin{array}{ll}
0 & 0 \\
0 & 1
\end{array}\right)
$$

and the Quarter-wave plate $\left(\Gamma=\frac{\pi}{2}\right)$

$$
\mathrm{W}_{\text {Quarter-wave plate }}=\frac{1}{\sqrt{2}}\left(\begin{array}{cc}
1 & -\mathrm{i} \\
-\mathrm{i} & 1
\end{array}\right)
$$

The Babinet-Soleil compensator in Fig. 2.12 is a device that can impart continuously variable phase retardation on the beam. It is basically composed of two calcite wedges that have their axes oriented at $90^{\circ}$. The overlap of the wedges can be adjusted thereby changing the propagation length through the wedges, which varies the amount of phase retardation imparted to the beam. The Babinet-Soleil compensator will be used in the experiments in Chapter 5 to vary the transmitted irradiance through the YCOB modulators while a fixed voltage is applied to the YCOB crystal. The particular BabinetSoleil compensator used in our experiments could produce phase retardation from $\mathbf{0}$ to $6 \pi$. The Jones matrix for the Babinet-Soleil compensator in terms of the compensator's adjustable retardation $\left(\Gamma_{c}\right)$ is

$$
W_{\text {compensator }}=e^{i \phi_{c}}\left(\begin{array}{cc}
\cos \frac{\Gamma_{c}}{2} & -i \sin \frac{\Gamma_{c}}{2} \\
-i \sin \frac{\Gamma_{c}}{2} & \cos \frac{\Gamma_{c}}{2}
\end{array}\right)
$$


The output polarizer has a Jones matrix given by

$$
W_{\text {output polarizer }}=\left(\begin{array}{ll}
1 & 0 \\
0 & 0
\end{array}\right)
$$

and the Jones vector for the input polarization ( $\overrightarrow{\mathrm{E}}_{\text {laser }}$ ) in Fig. 2.12 is

$$
\mathrm{E}_{\mathrm{laser}}=\frac{1}{\sqrt{2}}\left(\begin{array}{l}
0 \\
1
\end{array}\right)
$$

Now that we have the Jones matrices for all the optical elements in Fig. 2.12, we can calculate the output polarization $\left(E_{\text {out }}=\left(\begin{array}{l}E_{\text {y out }} \\ E_{\text {zout }}\end{array}\right)\right)$ by multiplying the matrices in the following way

$$
\left(\begin{array}{c}
E_{\text {yout }} \\
E_{\text {zout }}
\end{array}\right)=W_{\text {output polarizer }} W_{\text {compensator }} W_{x \text { xtal }} W_{\text {Quarter-wave plate }} W_{\text {input polarizer }} E_{\text {laser }}
$$

Multiplying the above matrices according to Eqn. (2.142) gives

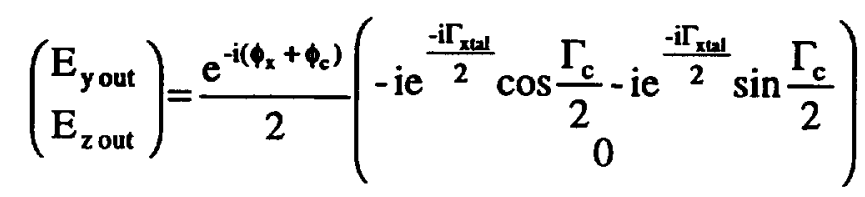

The transmitted irradiance $\left(I_{t r x}\right)$ through the modulator in Fig. 2.12 (i.e. through the output polarizer) can be calculated in MKS units using $I_{t x x}=\frac{\varepsilon_{0} c}{2}\left(\left|E_{y \text { out }}\right|^{2}+\left|E_{z \text { out }}\right|^{2}\right)$ as 


$$
I_{\mathrm{txx}}=\frac{\varepsilon_{0} c}{4}\left(1+\sin \Gamma_{c} \cos \Gamma_{x \mathrm{xal}}\right)=\frac{\varepsilon_{0} c}{4}\left(1+\sin \Gamma_{\mathrm{c}} \cos \left\{\frac{2 \pi L}{\lambda}\left(\left(\mathrm{n}_{\mathrm{z}}-\mathrm{n}_{\mathrm{y}}\right)-\frac{\left(\mathrm{r}_{33} \mathrm{n}_{\mathrm{z}}^{3}-\mathrm{r}_{23} \mathrm{n}_{\mathrm{y}}^{3}\right) \mathrm{V}_{\text {applied }}}{2 \mathrm{~d}}\right)\right\}\right)
$$

This equation describes the transmitted irradiance through the modulator in terms of the electro-optic coefficients and the voltage applied to the crystal. If we write Eqn. (2.131) as

$$
\Gamma_{x t a l}=\frac{2 \pi L}{\lambda}\left(\left(n_{z}-n_{y}\right)-\frac{\left(\Gamma_{33} n_{z}^{3}-\Gamma_{23} n_{y}^{3}\right) V_{\text {applied }}}{2 d}\right) \equiv \Gamma_{s}+\Gamma_{v}
$$

Then Eqn. (2.144) can be written as

$$
I_{\mathrm{trx}}=\frac{\varepsilon_{\mathrm{o}} \mathrm{c}}{4}\left(1+\sin \Gamma_{\mathrm{c}} \cos \left(\Gamma_{\mathrm{s}}+\Gamma_{\mathrm{v}}\right)\right)
$$

It is reasonable to assume that $V_{\text {applied }}<<V_{\boldsymbol{\pi}}$ (typical half-wave voltages for crystals range between $10 \mathrm{kV}-100 \mathrm{kV}$ and our experiments will use voltages between $20 \mathrm{~V}$ and $3 \mathrm{kV}$ ) in which case Eqn. (2.146) becomes

$$
I_{t r x}=\frac{\varepsilon_{0} c}{4}\left(1+\sin \Gamma_{c} \cos \Gamma_{s}-\sin \Gamma_{c} \sin \Gamma_{s} \frac{L}{d} \frac{\pi\left(r_{33} n_{z}^{3}-\Gamma_{23} n_{y}^{3}\right) V_{\text {applied }}}{\lambda}\right) \text { for } V_{\text {applied }} \ll V_{\pi}
$$

Note that Eqn. (2.147) implies that the transmitted irradiance $\left(I_{t r x}\right)$ is a linear function of applied voltage $\left(\mathrm{V}_{\text {applied }}\right)$.

There are three other YCOB crystal orientations that could be utilized in a transverse electro-optic modulator. We want to know $I_{t r x}$ for the modulators with the crystal in these 
three other orientations since they will also be experimentally investigated in Chapter 5 . A similar procedure to that just given in deriving Eqn. (2.147) can be used to find $I_{t r x}$ for these three other transverse YCOB modulators. Table 2.1 summarizes the results for all four transverse YCOB modulators. The quantities $n_{p}^{\prime \prime}$ and $n_{q}^{\prime \prime}$ in Table 2.1 are the electric field $\left(\mathrm{V}_{\text {applied }}\right)$ modified values of the relevant principal refractive indices as described in Eqns. (2.122) - (2.124) and as used in Eqn. (2.129). $\Gamma_{\mathrm{c}}$ is the Babinet-Soleil compensator retardation and $\Gamma_{3}$ is the crystal's static birefringence as defined in Eqn. (2.145). The crystal orientations ( $L$ and $d$ ) are given in terms of the crystal's dielectric directions. Note that only orientations along the crystal's dielectric axes will be studied. The equations for the transmitted irradiances $\left(I_{t a x}\right)$ given in Table 2.1 will be used to analyze the data collected on the four transverse $\mathrm{YCOB}$ modulators in Chapter 5. 
Table 2.1. Modified refractive indices, crystal retardations, and $I_{t r x}$ for four transverse $Y C O B$ modulators.

\begin{tabular}{|c|c|c|c|}
\hline $\begin{array}{l}\text { Crystal } \\
\text { Orientation } \\
\text { L:[xyz] } \\
\text { d:[xyz] }\end{array}$ & $\mathrm{n}_{\mathrm{p}}^{\prime \prime}$ & $\Gamma_{\mathrm{xtal}}=\frac{2 \pi \mathrm{L}}{\lambda}\left(\mathrm{n}_{\mathrm{p}}^{\prime \prime}-\mathrm{n}_{\mathrm{q}}^{\prime \prime}\right)$ & $\left(\mathrm{V}_{\text {applied }} \ll<\mathrm{V}_{\pi}\right)$ \\
\hline $\begin{array}{l}\mathrm{L}:[100] \\
\mathrm{d}:[001]\end{array}$ & $\begin{array}{l}\mathrm{n}_{z}^{\prime \prime}=\mathrm{n}_{\mathrm{z}}-\frac{\mathrm{r}_{33} \mathrm{n}_{\mathrm{z}}^{3} \mathrm{E}}{2} \\
\mathrm{n}_{\mathrm{y}}^{\prime \prime}=\mathrm{n}_{\mathrm{y}}-\frac{\mathrm{r}_{23} \mathrm{n}_{\mathrm{y}}^{3} \mathrm{E}}{2}\end{array}$ & $\frac{2 \pi L}{\lambda}\left(n_{z}-n_{y}\right)+\frac{L}{d} \frac{\pi}{\lambda}\left(r_{23} n_{y}^{3}-r_{33} n_{z}^{3}\right) v_{\text {applied }}$ & $\frac{\varepsilon_{0} c}{4}\left(1+\sin \Gamma_{\mathrm{c}} \cos \Gamma_{\mathrm{s}}-\sin \Gamma_{\mathrm{c}} \sin \Gamma_{\mathrm{s}} \frac{\mathrm{L}}{\mathrm{d}} \frac{\pi}{\lambda}\left(\mathrm{r}_{23} \mathrm{n}_{\mathrm{y}}^{3}-\mathrm{r}_{33} \mathrm{n}_{\mathrm{z}}^{3}\right) \mathrm{N}_{\text {applied }}\right)$ \\
\hline $\begin{array}{l}\mathrm{L}:[010] \\
\mathrm{d}:[100]\end{array}$ & $\begin{array}{l}\mathrm{n}_{\mathrm{z}}^{\prime \prime}=\mathrm{n}_{\mathrm{z}}-\frac{\mathrm{r}_{31} \mathrm{n}_{\mathrm{z}}^{3} \mathrm{E}}{2} \\
\mathrm{n}_{\mathrm{x}}^{\prime \prime}=\mathrm{n}_{\mathrm{x}}-\frac{\mathrm{r}_{11} \mathrm{n}_{\mathrm{x}}^{3} \mathrm{E}}{2}\end{array}$ & $\frac{2 \pi L}{\lambda}\left(n_{z}-n_{x}\right)+\frac{L}{d} \frac{\pi}{\lambda}\left(r_{11} n_{x}^{3}-r_{31} n_{z}^{3}\right) V_{\text {applied }}$ & $\frac{\varepsilon_{0} c}{4}\left(1+\sin \Gamma_{c} \cos \Gamma_{s}-\sin \Gamma_{c} \sin \Gamma_{s} \frac{L}{d} \frac{\pi}{\lambda}\left(r_{11} n_{x}^{3}-r_{31} n_{z}^{3}\right) V_{\text {applied }}\right)$ \\
\hline $\begin{array}{l}\text { L:[010] } \\
\mathrm{d}:[001]\end{array}$ & $\begin{array}{l}\mathrm{n}_{\mathrm{z}}^{\prime \prime}=\mathrm{n}_{\mathrm{z}}-\frac{\mathrm{r}_{33} \mathrm{n}_{\mathrm{z}}^{3} \mathrm{E}}{2} \\
\mathrm{n}_{\mathrm{x}}^{\prime \prime}=\mathrm{n}_{\mathrm{x}}-\frac{\mathrm{r}_{13} \mathrm{n}_{\mathrm{x}}^{3} \mathrm{E}}{2}\end{array}$ & $\frac{2 \pi L}{\lambda}\left(n_{z}-n_{x}\right)+\frac{L}{d} \frac{\pi}{\lambda}\left(r_{13} n_{x}^{3}-r_{33} n_{z}^{3}\right) V_{\text {applied }}$ & $\frac{\varepsilon_{o} c}{4}\left(1+\sin \Gamma_{c} \cos \Gamma_{s}-\sin \Gamma_{c} \sin \Gamma_{s} \frac{L}{d} \frac{\pi}{\lambda}\left(r_{13} n_{x}^{3}-r_{33} n_{z}^{3}\right) V_{\text {applied }}\right)$ \\
\hline $\begin{array}{l}\mathrm{L}:[001] \\
\mathrm{d}:[100]\end{array}$ & $\begin{array}{l}\mathrm{n}_{\mathrm{y}}^{\prime \prime}=\mathrm{n}_{\mathrm{y}}-\frac{\mathrm{r}_{21} \mathrm{n}_{\mathrm{y}}^{3} \mathrm{E}}{2} \\
\mathrm{n}_{\mathrm{x}}^{\prime \prime}=\mathrm{n}_{\mathrm{x}}-\frac{\mathrm{r}_{11} \mathrm{n}_{\mathrm{x}}^{3} \mathrm{E}}{2}\end{array}$ & $\frac{2 \pi L}{\lambda}\left(n_{y}-n_{x}\right)+\frac{L}{d} \frac{\pi}{\lambda}\left(r_{11} n_{x}^{3}-r_{21} n_{y}^{3}\right) V_{\text {applied }}$ & $\frac{\varepsilon_{0} c}{4}\left(1+\sin \Gamma_{c} \cos \Gamma_{s}-\sin \Gamma_{c} \sin \Gamma_{s} \frac{L}{d} \frac{\pi}{\lambda}\left(r_{11} n_{x}^{3}-r_{21} n_{y}^{3}\right) V_{\text {applied }}\right)$ \\
\hline
\end{tabular}




\subsubsection{Longitudinal YCOB modulators}

Another modulator configuration we will study in Chapter 5 is a longitudinal configuration. A longitudinal configuration is one in which the propagation direction of the beam and the applied electric field $\left(\mathrm{V}_{\text {applied }}\right)$ are parallel. An example of a longitudinal modulator we will consider is shown in Fig. 2.14. We would like to know the transmitted irradiance $\left(I_{t r x}\right)$ through this modulator for two orientations of the YCOB crystal. We will not show the derivation of $I_{t r x}$ for the two longitudinal cases but the procedure is very similar to that in deriving $I_{t x x}$ for the transverse cases. We give the results in Table 2.2. Note that for the longitudinal cases $I_{t r x}$ is independent of the crystal length. The quantities $n_{p}^{\prime \prime}$ and $n_{q}^{\prime \prime}$ are the electric field $\left(V_{\text {applied }}\right)$ modified values for the relevant principal refractive indices. $\Gamma_{c}$ is the Babinet-Soleil compensator retardation and $\Gamma_{s}$ is the crystal's static birefringence similar to that defined in Eqn. (2.145). The crystal orientations ( $\mathrm{L}$ and $\mathrm{d}$ ) are given in terms of the crystal's dielectric directions. The equations for the transmitted irradiances $\left(\mathrm{I}_{\mathrm{trx}}\right)$ given in Table 2.2 will be used to analyze the data collected on the two longitudinal YCOB modulators in Chapter 5. 


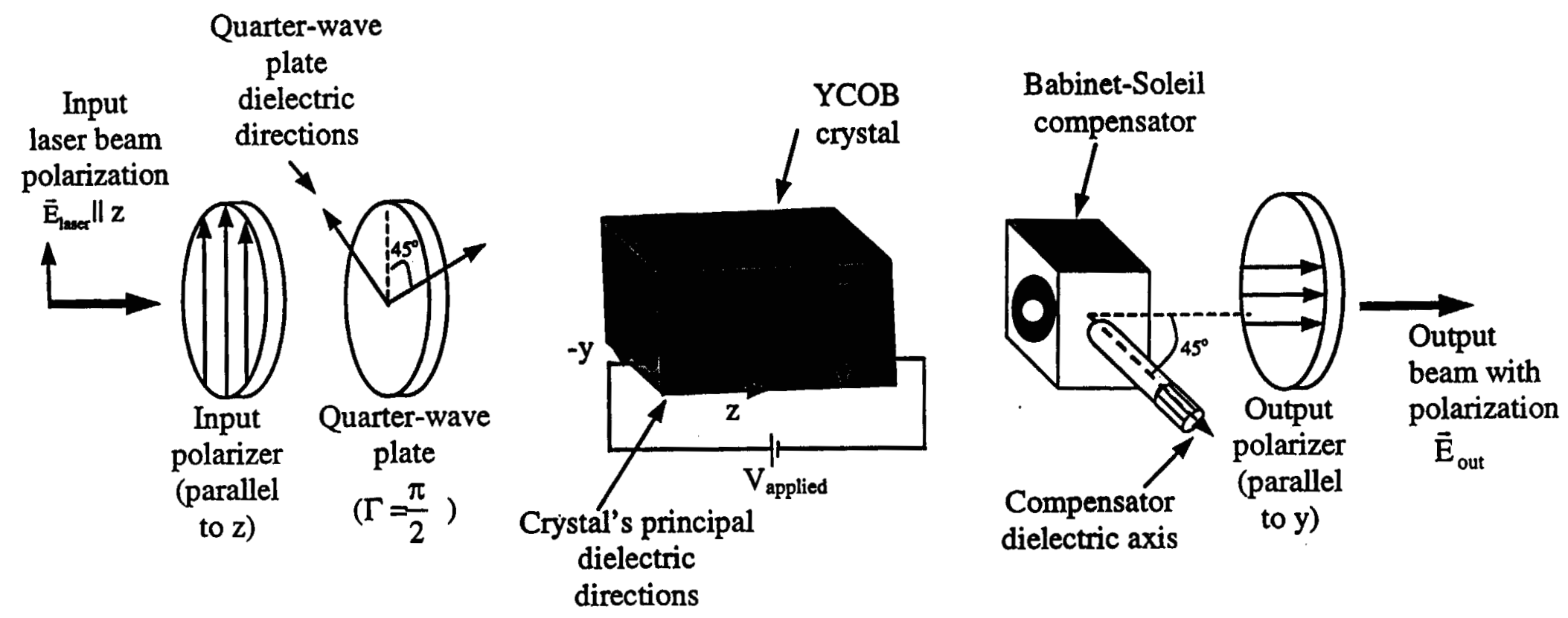

Figure 2.14. A longitudinal electro-optic YCOB modulator. In this case, the beam propagates along the crystal's $\mathrm{z}$ dielectric axis and the applied voltage is also along the $\mathrm{z}$ dielectric axis. 
Table 2.2. Modified refractive indices, crystal retardations, and $\mathrm{I}_{\mathrm{trx}}$ for two longitudinal $\mathrm{YCOB}$ modulators.

\begin{tabular}{|c|c|c|c|}
\hline $\begin{array}{l}\text { Crystal } \\
\text { Orientation } \\
\mathrm{L}:[\mathrm{xyz}] \\
\mathrm{d}:[\mathrm{xyz}]\end{array}$ & $\begin{array}{l}\mathrm{n}_{\mathrm{p}}^{\prime \prime} \\
\mathrm{n}_{\mathrm{q}}^{\prime \prime}\end{array}$ & $\Gamma_{x t a l}=\frac{2 \pi L}{\lambda}\left(n_{p}^{\prime \prime}-n_{q}^{\prime \prime}\right)$ & $\left(\mathrm{V}_{\text {applied }} \ll<\mathrm{V}_{\pi}\right)$ \\
\hline $\begin{array}{l}\mathrm{L}:[001] \\
\mathrm{d}:[001]\end{array}$ & $\begin{array}{l}\mathrm{n}_{\mathrm{y}}^{\prime \prime}=\mathrm{n}_{\mathrm{y}}-\frac{\mathrm{r}_{23} \mathrm{n}_{\mathrm{y}}^{3} \mathrm{E}}{2} \\
\mathrm{n}_{\mathrm{x}}^{\prime \prime}=\mathrm{n}_{\mathrm{x}}-\frac{\mathrm{r}_{13} \mathrm{n}_{\mathrm{x}}^{3} \mathrm{E}}{2}\end{array}$ & $\frac{2 \pi L}{\lambda}\left(n_{y}-n_{x}\right)+\frac{\pi}{\lambda}\left(r_{13} n_{x}^{3}-r_{23} n_{y}^{3}\right) N_{\text {applied }}$ & $\frac{\varepsilon_{0} c}{4}\left(1+\sin \Gamma_{c} \cos \Gamma_{s}-\sin \Gamma_{c} \sin \Gamma_{s} \frac{\pi}{\lambda}\left(\Gamma_{13} n_{x}^{3}-r_{23} n_{y}^{3}\right) V_{\text {applied }}\right)$ \\
\hline $\begin{array}{l}\mathrm{L}:[100] \\
\mathrm{d}:[100]\end{array}$ & $\begin{array}{l}\mathrm{n}_{z}^{\prime \prime}=\mathrm{n}_{z}-\frac{\mathrm{r}_{31} \mathrm{n}_{\mathrm{z}}^{3} \mathrm{E}}{2} \\
\mathrm{n}_{\mathrm{y}}^{\prime \prime}=\mathrm{n}_{\mathrm{y}}-\frac{\mathrm{r}_{21} \mathrm{n}_{\mathrm{y}}^{3} \mathrm{E}}{2}\end{array}$ & $\frac{2 \pi L}{\lambda}\left(n_{z}-n_{y}\right)+\frac{\pi}{\lambda}\left(r_{21} n_{y}^{3}-r_{31} n_{z}^{3}\right) V_{\text {applied }}$ & $\frac{\varepsilon_{o} c}{4}\left(1+\sin \Gamma_{c} \cos \Gamma_{s}-\sin \Gamma_{c} \sin \Gamma_{s} \frac{\pi}{\lambda}\left(r_{21} n_{y}^{3}-r_{31} n_{z}^{3}\right) V_{\text {applied }}\right)$ \\
\hline
\end{tabular}




\subsection{Summary}

This chapter has discussed the fundamental concepts of nonlinear optics necessary to understand the experiments that will be conducted with the COB crystals. The nonlinear processes in the COB crystals that will be experimentally investigated in the next three chapters $(3-5)$ are second harmonic generation, optical parametric oscillation, and the linear electro-optic effect. A mathematical formulation of these processes has been described. The equations derived in this chapter will be used to analyze the experimental data collected in Chapters 3-5. 


\section{References for Chapter 2}

[1] R. Norrestam, M. Nygren, and J.-O. Bovin, Chem. Mater. 4, 737 (1992)

[2] M. Iwai, T. Kobayashi, H. Furuya, Y. Mori, and T. Sasaki, Jpn. J. Appl. Phys. 36, L276 (1997)

[3] G. Aka, A. Kahn-Harari, F. Mougel, D. Vivien, F. Salin, P. Coquelin, P. Colin, D. Pelenc, and J. Damelet, J. Opt. Soc. Am. B 14, 2238 (1997)

[4] Q. Ye and B. H. T. Chai, J. Cryst. Growth 197, 228 (1999)

[5] R. W. Boyd, Nonlinear Optics, (Academic Press, San Diego, California, 1992)

[6] F. Zernike and J. E. Midwinter, Applied Nonlinear Optics, (John Wiley and Sons, Inc., New York, 1973)

[7] A. Yariv and P. Yeh, Optical Waves in Crystals - Propagation and Control of Laser Radiation, (John Wiley and Sons, Inc., New York, 1984)

[8] M. Born and E. Wolf, Principles of Optics, $7^{\text {th }}$ ed. (Cambridge University Press, Cambridge, United Kingdom, 1999)

[9] R. K. Wangsness, Electromagnetic Fields, (John Wiley and Sons, Inc., New York, 1986)

[10] E. E. Wahlstrom, Optical Crystallography, $4^{\text {th }}$ ed. (John Wiley and Sons, Inc., New York, 1951)

[11] F. Brehat and B. Wyncke, J. Phys. B: At. Mol. Opt. Phys., 22, 1891 (1989)

[12] D. A. Roberts, IEEE J. Quan. Elec., 28, 2057 (1992)

[13] A. Yariv, Quantum Electronics, $3^{\text {nd }}$ ed. (John Wiley and Sons, Inc., New York, 1989)

[14] P. D. Maker, R. W. Terhune, M. Nisenoff, and C. M. Savage, Phys. Rev. Lett., 8, 21 (1962)

[15] J. A. Giordmaine, Phys. Rev. Lett., 8, 19 (1962)

[16] M. V. Hobden, J. Appl. Phys., 38, 4365 (1967)

[17] V. G. Dmitriev, et al., Handbook of Nonlinear Optical Crystals, $3^{\text {rd }}$ ed. (SpringerVerlag, Berlin, 1999)

[18] H. P. Weber, E. Mathieu, and K. P. Meyer, J. Appl. Phys., 37, 3584 (1966)

[19] S. J. Brosnan and R. L. Byer, IEEE J. Quan. Elec., 15, 415 (1979)

[20] A. E. Siegman, Lasers, (University Science Books, Sausalito, California, 1986)

[21] J. F. Nye, Physical Properties of Crystals, 1985 ed. (Clarendon Press, Oxford, 1985)

[22] R. C. Jones, J. Opt. Soc. Am., 31, 488 (1941) 


\section{Chapter 3}

\section{Harmonic frequency conversion in $\mathrm{LaCa}_{4} \mathrm{O}\left(\mathrm{BO}_{3}\right)_{3}, \mathrm{GdCa}_{4} \mathrm{O}\left(\mathrm{BO}_{3}\right)_{3}$, $\mathrm{YCa}_{4} \mathrm{O}\left(\mathrm{BO}_{3}\right)_{3}$, and $\mathrm{Gd}_{0.275} \mathrm{Y}_{0.725} \mathrm{Ca}_{4} \mathrm{O}\left(\mathrm{BO}_{3}\right)_{3}$}

First published as: J. J. Adams, C. A. Ebbers, K. I. Schaffers, and S. A. Payne, "Nonlinear optical properties of $\mathrm{LaCa}_{4} \mathrm{O}\left(\mathrm{BO}_{3}\right)_{3}$," Optics Letters, 26, 217 (2001)

\subsection{Introduction}

The $\mathrm{GdCa}_{4} \mathrm{O}\left(\mathrm{BO}_{3}\right)_{3}(\mathrm{GdCOB})$ family of nonlinear optical crystals (i.e. $\mathrm{GdCOB}$, $\mathrm{LuCa}_{4} \mathrm{O}\left(\mathrm{BO}_{3}\right)_{3}(\mathrm{LuCOB}), \mathrm{YCa}_{4} \mathrm{O}\left(\mathrm{BO}_{3}\right)_{3}(\mathrm{YCOB})$, and $\mathrm{Gd}_{\mathrm{x}} \mathrm{Y}_{1-\mathrm{x}} \mathrm{Ca}_{4} \mathrm{O}\left(\mathrm{BO}_{3}\right)_{3}(\mathrm{Gd}, \mathrm{YCOB})$, etc.) are known to be capable of second harmonic conversion of near infrared wavelengths [1-4]. GdCOB and the related crystals are relatively easy to grow, are not hygroscopic, and are easy to polish and optically coat. These crystals have already found potential application for frequency doubling and tripling of large aperture, high peak, and high-average power lasers [5].

In a comparison of intracavity frequency doubling (SHG) of a Q-switched multimode laser operating at $1064 \mathrm{~nm}$, a GdCOB crystal produced $1 / 2$ the output power of an equivalent crystal of $\mathrm{LiB}_{3} \mathrm{O}_{5}(\mathrm{LBO})$ [6]. The reduction in SHG output was due to the approximately 5 times larger angular sensitivity of the critically phasematched GdCOB, leading to inefficient conversion of the higher order modes within the laser cavity. Intracavity frequency conversion requires a low angular sensitivity to allow efficient conversion of multimode sources, or to allow efficient conversion of continuous wave sources where a high quality beam mode is tightly focussed within the doubling crystal. Crystals capable of non-critically phasematched (NCPM) interactions are preferred in these instances because non-critical phasematching has inherently low angular sensitivity as was discussed in Chapter 2. 
YCOB and GdCOB have type II NCPM SHG wavelengths of 1.017 and $1.2 \mu \mathrm{m}$, respectively for propagation down the $y$ dielectric axis $[2,7]$. By growing the mixed crystal, $\mathrm{Gd}_{\mathrm{x}} \mathrm{Y}_{1-\mathrm{x}} \mathrm{Ca}_{4} \mathrm{O}\left(\mathrm{BO}_{3}\right)_{3}(\mathrm{Gd}, \mathrm{YCOB})$ with various ratios of gadolinium to yttrium, the non-critical wavelength can be tuned to a specific wavelength [8]. In general, the magnitude of the nonlinear coupling coefficient for non-critical type II interactions down the $y$ dielectric axis in the COB crystals can be estimated to be between $0.30-0.35 \mathrm{pm} / \mathrm{V}$ [2]. Alternatively, type I NCPM SHG in GdCOB and YCOB is possible for propagation down the $\mathrm{z}$ dielectric axis. The type I NCPM SHG wavelengths for GdCOB and YCOB are 966 and $840 \mathrm{~nm}$, respectively [9]. However, the magnitude of the nonlinear coupling coefficients $\left(d_{i l}\right)$ for these type I NCPM interactions is not known since only the effective coupling coefficients $\left(d_{\text {eff }}\right)$ for the critically phasematched processes have been measured $[2,3]$.

The growth and characterization of crystals isostructural to GdCOB and YCOB were explored for intracavity frequency conversion applications, with the goal of finding crystals with a smaller birefringence that would allow non-critical phasematching of wavelengths near $1047 \mathrm{~nm}, 1053 \mathrm{~nm}$, or $1064 \mathrm{~nm}$ (emission wavelengths of Nd:YLF and $\mathrm{Yb}^{3+}: \mathrm{Sr}_{5}\left(\mathrm{PO}_{4}\right)_{3} \mathrm{~F}(\mathrm{Yb}: \mathrm{S}-\mathrm{FAP}), \mathrm{Nd}$ :Phosphate glass, and Nd:YAG, respectively). LuCOB, YCOB, and $G d C O B$ exhibit decreasing $x z$ principal plane birefringence (i.e. $\left.\ln _{\mathbf{x}}-\mathbf{n}_{\mathbf{z}} l\right)$ with an increase in the mean radius of the lanthanide ion $[3,10]$. The decrease in birefringence with increasing ion size is believed to be due to the increase in ion size distorting the crystal structure and as a result causing the highly birefringent borate groups $\left(\mathrm{BO}_{3}\right)$ to change alignment $[11,12]$. We have exploited this trend of decreasing birefringence with increasing mean radius of the lanthanide ion to extend the type I non- 
critical wavelength as long as possible by completely substituting lanthanum for gadolinium in the GdCOB structure. (For the case of trivalent, octahedrally coordinated lanthanide ions, trivalent lanthanum has the largest mean radius [13].) Furthermore, because $\mathrm{La}^{3+}$ has a closed-shell electronic structure (i.e. no unpaired valence electrons) another attractive feature of incorporating it into the GdCOB structure is that the resulting compound $\left(\mathrm{LaCa}_{4} \mathrm{O}\left(\mathrm{BO}_{3}\right)_{3}\right)$ would have high transparency for wavelengths that extend from the visible down to its ultra-violet band edge.

In this chapter, we report the successful growth and characterization of the new nonlinear crystal $\mathrm{LaCa}_{4} \mathrm{O}\left(\mathrm{BO}_{3}\right)_{3}$ ( $\left.\mathrm{LaCOB}\right)$. We also experimentally compare the nonlinear optical properties of $\mathrm{LaCOB}, \mathrm{GdCOB}, \mathrm{YCOB}$, and $\mathrm{Gd}, \mathrm{YCOB}$ relative to a standard suite of nonlinear optical crystals $\left(\mathrm{KTiOPO}_{4}(\mathrm{KTP}), \mathrm{BaB}_{2} \mathrm{O}_{4}(\mathrm{BBO}), \mathrm{KD}_{2} \mathrm{PO}_{4}\right.$ (KD*P), and LBO). In section 3.2, we discuss the growth of $\mathrm{LaCOB}$ and the preparation of all the crystal samples studied in this chapter. Section 3.3 discusses the SHG experiments conducted with our set of ten crystals and presents the results for the effective coupling coefficients $\left(\mid \mathrm{d}_{\mathrm{eff}}\right)$ and angular sensitivities $\left(\beta_{\theta}\right)$. The calculation of two coefficients of the nonlinear optical tensor $\left(d_{12}\right.$ and $\left.d_{32}\right)$ for the COB crystals is also discussed. Section 3.4 describes results from experiments that estimate the temperature sensitivity $\left(\beta_{\mathrm{T}}\right)$ of type I SHG in LaCOB. Section 3.5 summarizes the chapter.

\subsection{Sample preparation}

Large crystals of LaCOB were obtained using the Czochralski pulling method with careful alignment of the seed crystal along the preferred growth direction (the y dielectric axis) [14]. The material melts congruently and was grown with a pull rate of $1 \mathrm{~mm} / \mathrm{hr}$ from a 3 inch diameter iridium crucible while rotating at $20 \mathrm{rpm}$. Typical growth sizes 
were $2.5-3.0 \mathrm{~cm}$ diameters $\times 10 \mathrm{~cm}$ in length. The crystals were clear with minimal bubble core defects [14] perpendicular to the growth direction. The crystal structure (monoclinic) and the location of the $y$ dielectric axis in the $\mathrm{LaCOB}$ boules were determined using $x$-ray diffraction at LLNL. $5 \mathrm{~mm}^{3}$ samples for type I SHG were then cut for analysis. Transmission spectra were collected on the LaCOB samples using a Schimadzu Corp. model 3101 UVPC spectrophotometer. An unpolarized transmission spectrum for an uncoated $5.15 \mathrm{~mm}$ thick sample of LaCOB is shown in Fig. 3.1. The ultra-violet band edge occurs at $\sim 200 \mathrm{~nm}$ and the features in the $2400-3200 \mathrm{~nm}$ range are a combination of absorption by the borate groups and water impurities in the crystal [2].

As was discussed in Chapter 2, the $\mathrm{COB}$ crystals are monoclinic biaxial crystals with point group $\mathrm{m}$ symmetry with the convention that $\mathrm{m} \perp \mathrm{b}$. The mutually orthogonal

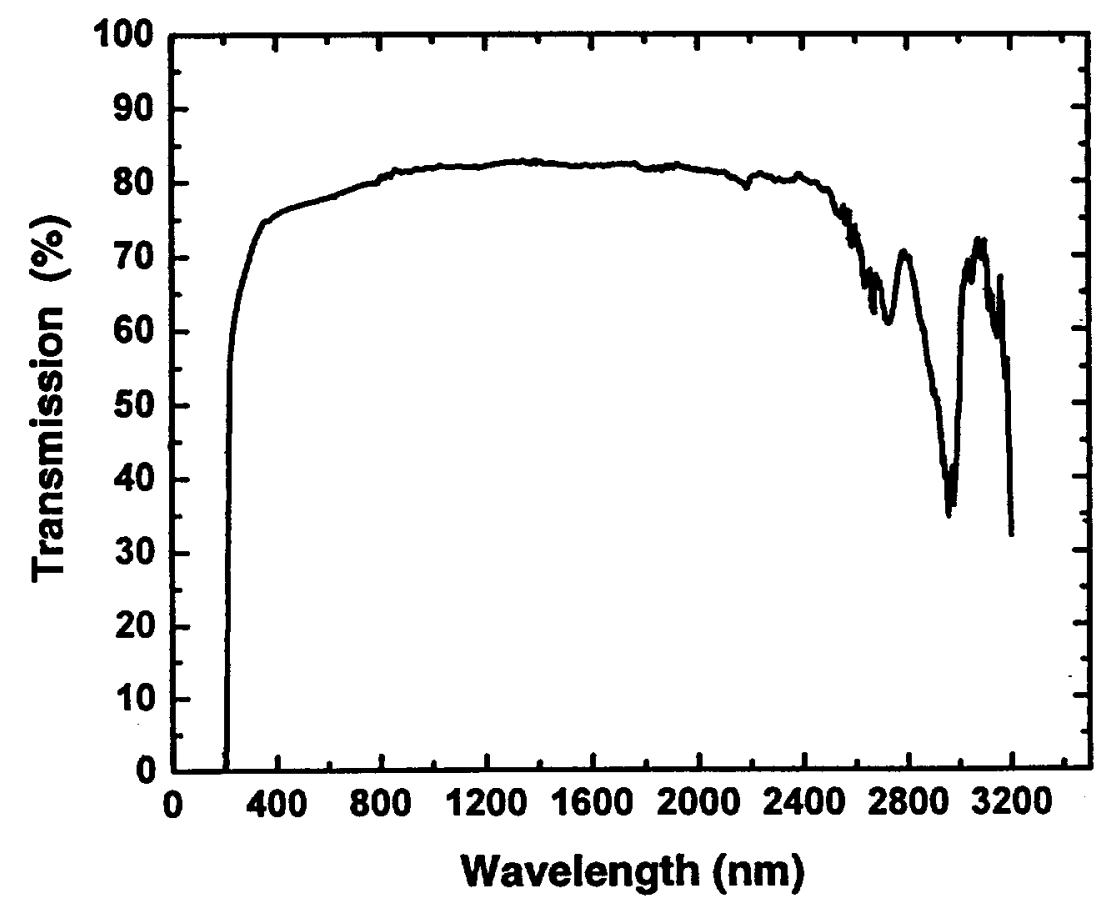

Figure 3.1. Unpolarized transmission spectrum for an uncoated $5.15 \mathrm{~mm}$ thick sample of $\mathrm{LaCOB}$ at room temperature. 
principal dielectric axes in these crystals are labeled $(x, y, z)$. The refractive indices for light polarized parallel to the $x, y$, or $z$ dielectric axes are $n_{x}, n_{y}$, and $n_{z}$, respectively, with the convention that $n_{x}<n_{y}<n_{z}$. The effective coupling coefficient ( $d_{\text {eff }}$ ) for type I SHG in the $\mathrm{xz}$ principal plane of this crystal class is given by

$$
d_{e f f}=d_{32} \cos \phi-d_{12} \sin \phi \quad \text { for } \phi>V
$$

where $\phi$ is the angle measured from the $x$ dielectric axis in the $x z$ plane, $d_{12}\left(d_{122}\right)$ and $d_{32}$ (d322) are coefficients of the nonlinear optical tensor, and $V$ is the optic angle measured from the acute bisectrix ( $\mathrm{x}$ dielectric axis).

A group of ten crystals were studied and the effective coupling coefficients $\left(d_{\text {eff }}\right)$ and angular sensitivities $\left(\beta_{\theta}\right)$ for SHG at $1064 \mathrm{~nm}$ were experimentally determined as shown in Table 3.1. The phasematching directions were taken from the literature or calculated using Sellmeier equations found in the literature $[2,3,15,16]$. The angles specified for the different crystals in Table 3.1 are according to the conventions discussed in Chapter 2. The various samples were obtained as follows: $5 \mathrm{~mm}^{3}$ cubes of YCOB were cut from a Czochralski grown boule (Crystal Photonics, Fl) at $+57^{\circ}$ and $-57^{\circ}$ (relative sign) from the $x$ dielectric axis in the $x z$ plane. The faces of these $Y C O B$ cubes were uncoated and polished to within 30 seconds of parallel. The $5.09 \mathrm{~mm}$ GdCOB sample was commercially grown (Crismatec, France), antireflection coated, and cut for maximum second harmonic conversion at $1064 \mathrm{~nm}$ in the $\mathrm{xz}$ plane. A $15 \mathrm{~mm}$ long commercially grown sample of GdCOB, originally cut for maximum conversion, was subsequently recut and polished at LLNL, producing a $3.06 \mathrm{~mm}$ sample for phasematching on the opposite side of the $\mathrm{x}$ dielectric axis in the $\mathrm{xz}$ plane. We procured a Czochralski grown 
boule of $\mathrm{Gd}_{\mathrm{x}} \mathrm{Y}_{1-\mathrm{x}} \mathrm{Ca}_{4} \mathrm{O}\left(\mathrm{BO}_{3}\right)_{3}$ from Crystal Photonics Inc, FL. The Gd to $\mathrm{Y}$ ratio $(\mathrm{x}=$ 0.275) was adjusted so that type II non-critical phasematching at $1064 \mathrm{~nm}$ occurred for propagation down the $y$ dielectric axis. A single $3.75 \mathrm{~mm}$ sample of LaCOB was prepared with faces approximately perpendicular to the phasematching direction for type I SHG at $1064 \mathrm{~nm}$ in the Xz plane. Phasematched type I SHG at $1064 \mathrm{~nm}$ in LaCOB was subsequently determined to occur at 80.1 degrees from the $\mathrm{x}$ dielectric axis in the $\mathrm{xz}$ principal plane.

The nonlinear crystals we used as reference standards were KTP (Crystal Associates, NJ), BBO (Inrad, NJ), KD*P (Inrad, NJ), and LBO (Fujian Castech, PRC). These crystals were commercially grown and all antireflection coated except for the KD*P.

\subsection{Angle-tuned phasematched SHG experiments}

\subsection{Effective coupling coefficients $\left(d_{e f f}\right)$ and angular sensitivities $\left(\beta_{\theta}\right)$}

The effect of wavevector mismatch on the generated $2 \omega$ irradiance for all 10 of the crystals shown in Table 3.1 was measured as a function of angle from the phasematching direction. We measured $\mathrm{I}_{2 \omega}(\Delta \theta)$ vs. $\Delta \theta$ using the experimental apparatus shown in Fig. 3.2. We employed a polarized $0.5 \mathrm{~W}$ single transverse mode CW Nd:YAG (1064 nm) laser and synchronous lock-in amplifier detection. The quartz polarization rotator was used to rotate the polarization of the Nd:YAG laser from horizontal to vertical. We collected $I_{2 \omega}(\Delta \theta)$ vs. $\Delta \theta$ data on our collection of crystals, systematically repeating the measurements until we had collected four complete data sets. Fig. 3.3 shows an example of the SHG data collected on the $3.75 \mathrm{~mm}$ LaCOB sample. Note how the data in Fig. 3.3 clearly reveals the $\operatorname{sinc}^{2}$ behavior predicted by Eqn. (2.52). Fig. 3.4 shows examples of 


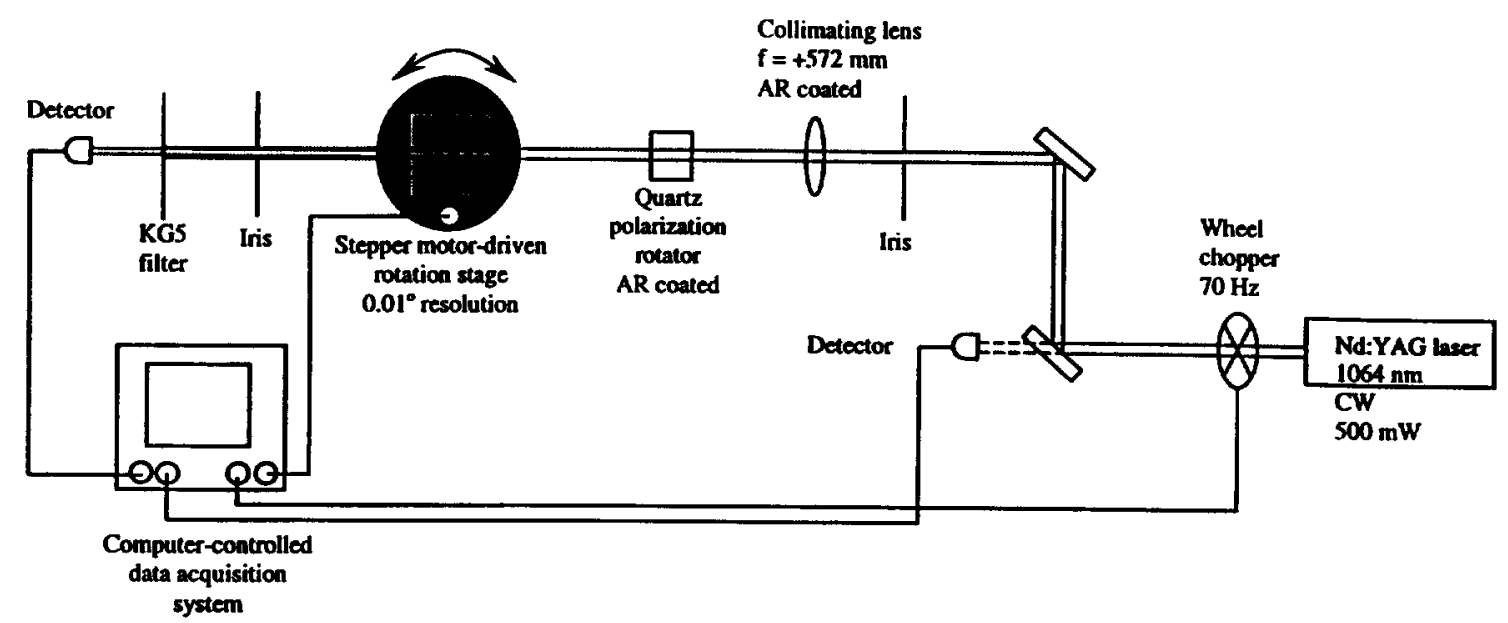

Figure 3.2. Experimental setup to measure the SHG response as a function of angle.

the SHG data collected on the $5.11 \mathrm{~mm}$ YCOB sample and the $5.09 \mathrm{~mm}$ GdCOB sample.

The LaCOB data is shown again in Fig. 3.4 for comparison. The form of the curves in

Figs. 3.3 and 3.4 is typical of the data collected for all the crystals. The curves in Fig. 3.4

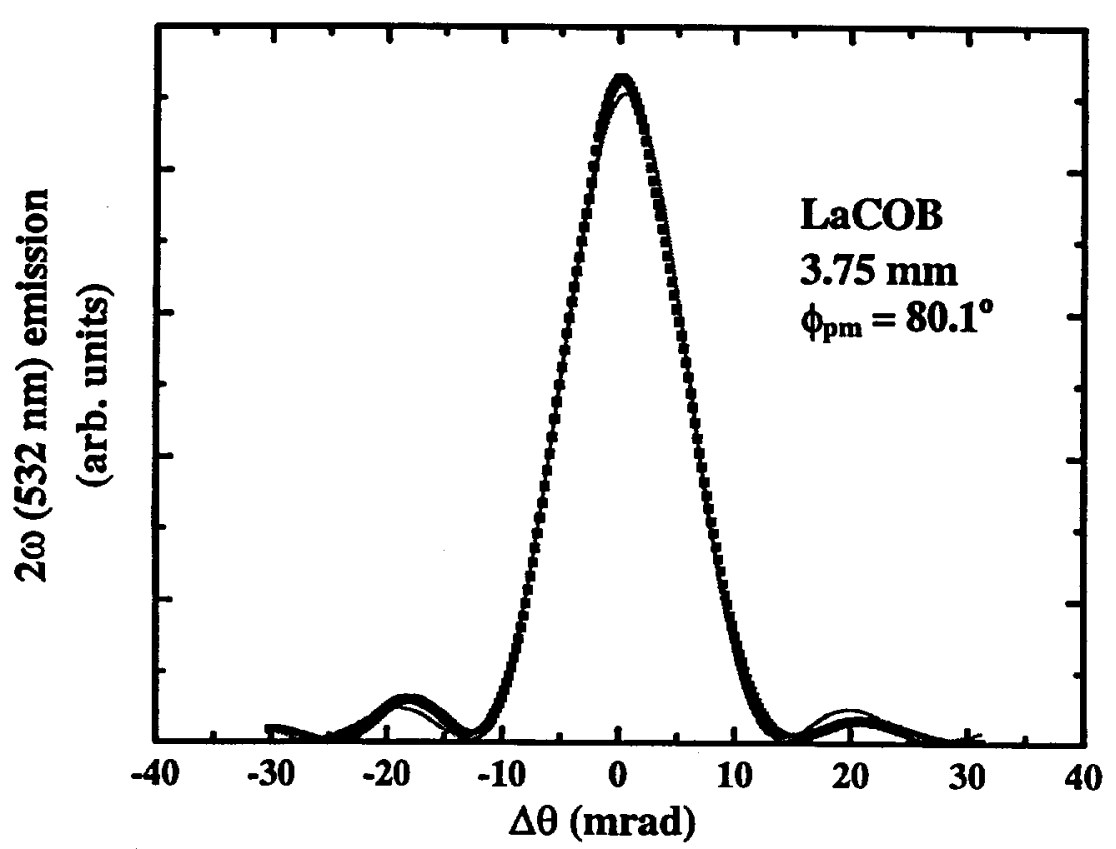

Figure 3.3. $2 \omega$ emission for type I SHG at $1064 \mathrm{~nm}$ as a function of angle from the phasmatching direction in the $x z$ plane for $\mathrm{LaCOB} . \phi_{\mathrm{pm}}$ is the phasematching angle measured from the $x$ dielectric axis. 


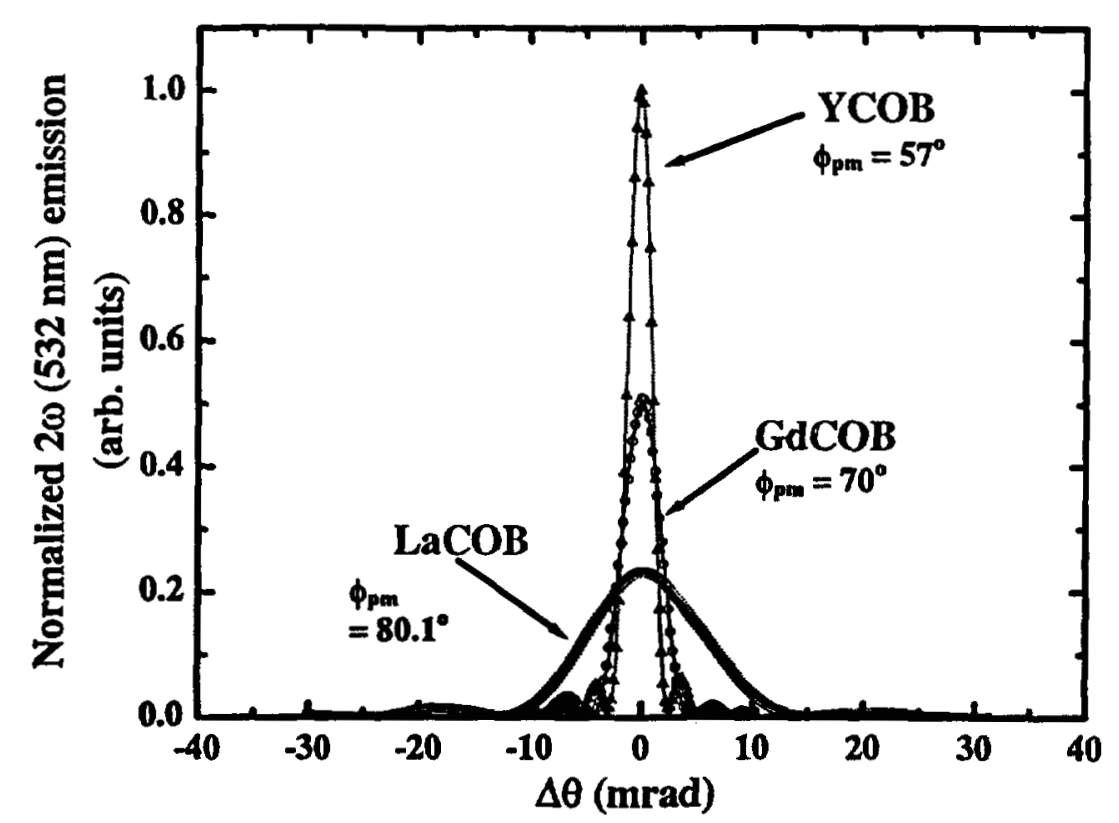

Figure 3.4. Normalized $2 \omega$ emission for type I SHG at $1064 \mathrm{~nm}$ as a function of angle from the phasmatching direction in the xz plane for $\mathrm{LaCOB}, \mathrm{GdCOB}$, and YCOB. $\phi_{p m}$ is the phasematching angle measured from the $\mathrm{x}$ axis for each crystal.

have been normalized by the value of the peak response for the $\mathrm{YCOB}$ crystal and by the square of the lengths of the samples. Therefore the ratio of the peaks in Fig. 3.4 represents the square of the ratio of the corresponding $\left|d_{\text {eff }}\right| s$. The lines through each of the data sets in Figs. 3.3 and 3.4 are the corresponding numerical fits of Eqn. (2.52). Though the peak $2 \omega$ response of $\mathrm{LaCOB}$ is the smallest of the three crystals, its angular sensitivity (i.e. the inverse of the widths in Fig. 3.4) can be seen to be about one-half that of GdCOB and one-third that of YCOB.

The absolute values for the $d_{e f f}$ 's given in Table 3.1 for all of the crystals except KTP were calculated from the ratio [15] of the peak $2 \omega$ signal produced by each sample and that of KTP $\left(d_{e f f}=3.2 \mathrm{pm} / \mathrm{V}[16]\right)$. Our $\mathrm{d}_{\text {eff }}$ values for $\mathrm{BBO}$ and $\mathrm{KD} * \mathrm{P}$ each agree within $1 \%$ of the values found by Eckhardt, et al. [16]. The $d_{\text {eff }}$ value for $L B O$ agrees within $2 \%$ of that found by Velsko, et al. [15]. Given the excellent agreement between our 
Table 3.1. Experimentally determined nonlinear crystal properties for SHG at $1064 \mathrm{~nm}$.

\begin{tabular}{|c|c|c|c|c|}
\hline $\begin{array}{c}\text { Crystal, Type } \\
\text { of } \\
\text { Phasematching }\end{array}$ & $\begin{array}{l}\text { Length } \\
(\mathrm{mm})\end{array}$ & $\begin{array}{l}\text { Phase-matching } \\
\text { Direction } \\
(\theta, \phi) \text { (degrees) }\end{array}$ & $\begin{array}{c}\mathrm{dd}_{\mathrm{eff}} \mathrm{t} \\
(\mathrm{pm} / \mathrm{V})\end{array}$ & $\begin{array}{c}\text { Angular } \\
\text { Sensitivity } \\
\beta_{\theta} \\
(\mathrm{cm}-\mathrm{rad})^{-1}\end{array}$ \\
\hline KTP,II & 5.17 & $(65.7,90)$ & 3.2 & $573 \pm 56$ \\
\hline BBO,I & 6.78 & $(22.8,0)$ & $1.97 \pm 0.12$ & $10110 \pm 1082$ \\
\hline $\mathbf{K D} * \mathbf{P}, \mathbf{I}$ & 29.96 & $(36.6,45)$ & $0.21 \pm 0.02$ & $3455 \pm 511$ \\
\hline LBO,II & 5.43 & $(69.6,90)$ & $0.67 \pm 0.05$ & $562 \pm 25$ \\
\hline GdCOB,I & 5.09 & $(90,-70)^{\dagger}$ & $0.78 \pm 0.06$ & $2704 \pm 156$ \\
\hline GdCOB,I & 3.06 & $(90,70)^{\dagger}$ & $0.38 \pm .04$ & $2690 \pm 161$ \\
\hline YCOB,I & 5.11 & $(90,-57)^{\dagger}$ & $1.12 \pm 0.07$ & $4548 \pm 277$ \\
\hline YCOB,I & 5.10 & $(90,57)^{\dagger}$ & $0.69 \pm 0.05$ & $4385 \pm 440$ \\
\hline LaCOB,I & 3.75 & $(90,-80.1)$ & $0.52 \pm 0.05$ & $1224 \pm 184$ \\
\hline $\begin{array}{l}\text { Gd,YCOB, } \\
\text { II NCPM }\end{array}$ & 3.06 & $(0,0)$ & $0.37 \pm 0.04$ & $145 \pm 36$ \\
\hline
\end{tabular}

For each set of materials, the choice for the relative sign of the angles is arbitrary and is only meant to differentiate between the relative orientation of the samples.

measurements of $d_{e f f}$ for $B B O, K D * P$, and LBO, and those found in the literature, we believe our method for determining $d_{e f f}$ 's to be highly accurate.

The angular sensitivity $\left(\beta_{\theta}\right)$ for each crystal in Table 3.1 was determined from a numerical fit of Eqn. (2.52) $\left(\Delta k=\beta_{\theta} \Delta \theta\right)$ to the corresponding data set. The angular sensitivity for type I SHG at $1064 \mathrm{~nm}$ in $\mathrm{LaCOB}$ is determined to be about one-half that of GdCOB and about twice that of KTP or LBO. Actually, since the angular sensitivity determined for LBO is for a type II process the corresponding angular sensitivity for a type I process in LBO would have approximately the same angular sensitivity as LaCOB. (A type II process has an angular sensitivity that is one-half the angular sensitivity of the 
corresponding type I process. This can be seen by comparing the derivatives of $\Delta \mathrm{k}$ with respect to angle $(\Delta \theta)$ for the type I and type II processes [17].)

We also conducted type I SHG experiments at $1047 \mathrm{~nm}$ with LaCOB in order to evaluate its type I NCPM wavelength for propagation down the $\mathrm{z}$ dielectric axis. By knowing the phasmatching angles for SHG at 1047 and $1064 \mathrm{~nm}$, we will be able to determine the NCPM wavelength through extrapolation (as we will discuss shortly). The data in Fig. 3.5 for $\mathrm{LaCOB}$ at $1047 \mathrm{~nm}$ was measured by replacing the Nd:YAG laser in Fig. 3.2 with a $0.35 \mathrm{~W}$ Nd:YLF laser. The angle for maximum SHG at $1047 \mathrm{~nm}$ in $\mathrm{LaCOB}$ was determined to be $84.5^{\circ}$ from the $\mathrm{x}$ dielectric axis in the $\mathrm{xz}$ plane. The data for LaCOB in Fig. 3.5 allows us to determine values for $d_{\text {eff }}$ and $\beta_{\theta}$ for type I SHG at

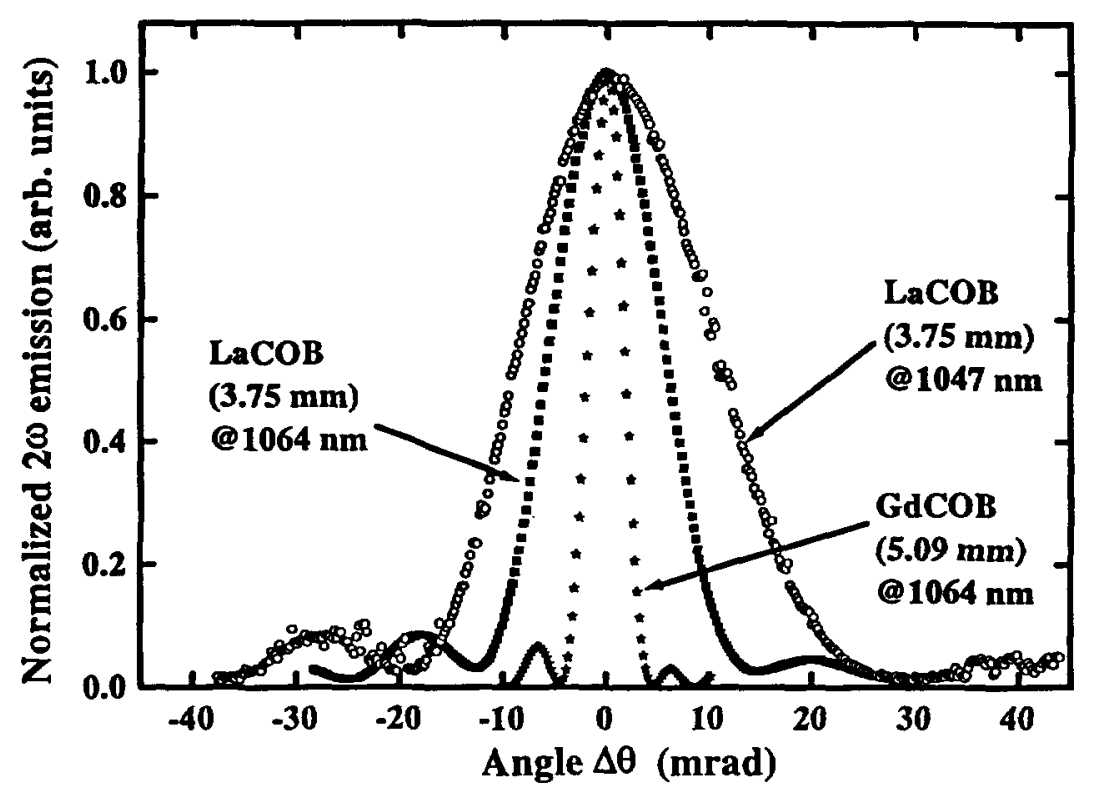

Figure 3.5. Normalized $2 \omega$ emission for type I SHG at 1047 and $1064 \mathrm{~nm}$ in LaCOB and for type I SHG at $1064 \mathrm{~nm}$ in GdCOB as a function of angle from the phasematching directions in the $x z$ plane.

$1047 \mathrm{~nm}$. We determine a $\mathrm{d}_{\mathrm{eff}}$ and $\beta_{\theta}$ of $0.37 \pm 0.04 \mathrm{pm} / \mathrm{V}$ and $716 \pm 107(\mathrm{~cm}-\mathrm{rad})^{-1}$, respectively for type I SHG at $1047 \mathrm{~nm}$ in the $\mathrm{xz}$ plane of LaCOB. Notice that as the 
magnitude of the phasematching angle increases in $\mathrm{LaCOB}$ (i.e. $\phi=80.1^{\circ}$ for $1064 \mathrm{~nm}$ SHG and $\phi=84.5^{\circ}$ for $1047 \mathrm{~nm}$ SHG) the angular sensitivity $\left(\beta_{\theta}\right)$ decreases (i.e. the widths of the curves in Fig. 3.5 increases). This decrease in the angular sensitivity $\left(\beta_{\theta}\right)$ occurs because the interaction is getting closer to being NCPM (i.e. $\phi=90^{\circ}$ ).

Fig. 3.6 shows the change in the type I phasematched SHG wavelength as a function of angle from the $x$ dielectric axis for $\mathrm{LaCOB}, \mathrm{GdCOB}$, and YCOB. The curves for GdCOB and YCOB were calculated from their respective Sellmeier equations from Ref. [3]. The two points for LaCOB at $80.1^{\circ}$ and $84.5^{\circ}$ were experimentally measured. The point at $90^{\circ}$ was then extrapolated by fitting a phasematching curve to the $\mathrm{LaCOB}$ data

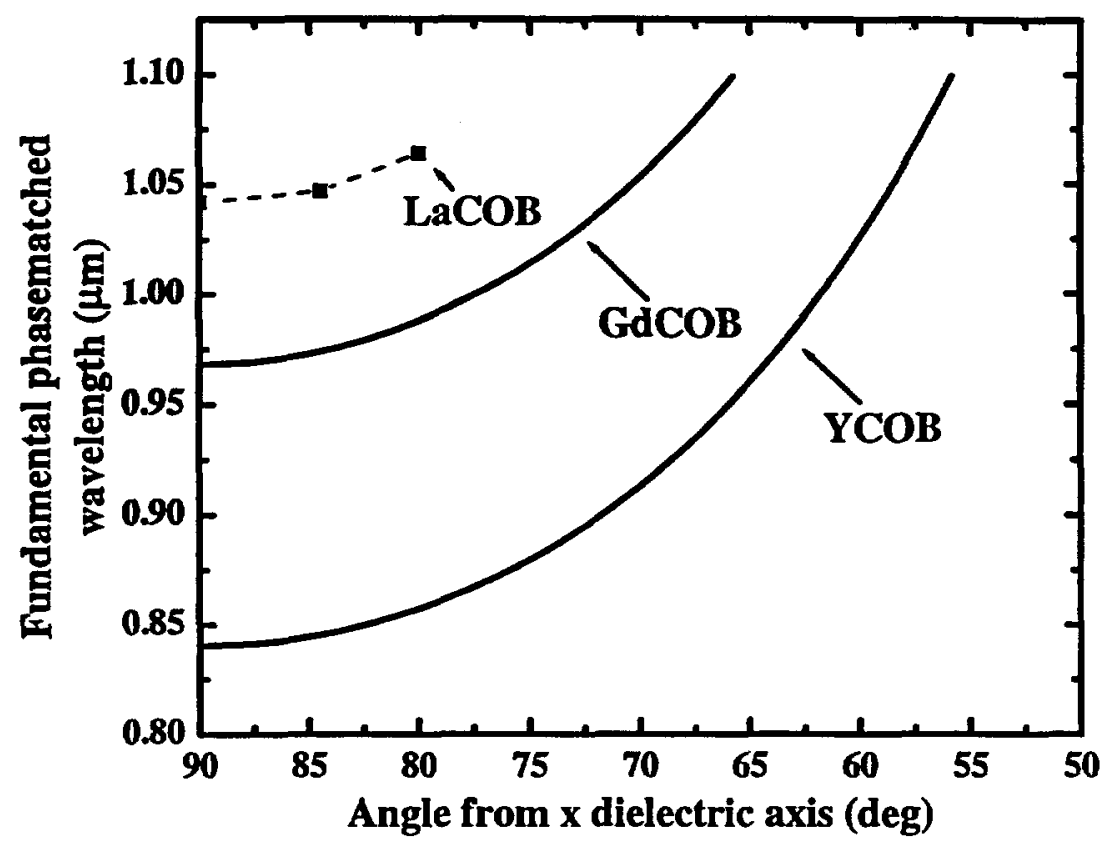

Figure 3.6. The change in the type I phasematched SHG wavelength as a function of angle from the $x$ dielectric axis for $\mathrm{LaCOB}, \mathrm{GdCOB}$, and YCOB.

calculated using the Sellmeier equations for GdCOB with an adjusted birefringence. Therefore from the extrapolated $y$-intercept for LaCOB shown in Fig. 3.6, we estimate the type I NCPM SHG wavelength to be $1042 \pm 1.5 \mathrm{~nm}$. 


\subsubsection{Calculation of the absolute nonlinear coupling coefficients ( $d_{12}$ and $d_{32}$ )}

Because of the symmetry of the index ellipsoid, phasematching occurs for type I interactions in the $x z$ principal plane of the $C O B$ crystals along two symmetric propagation directions, $+\phi$ and $-\phi$. As can be seen from Eqn. (3.1), the effective coupling coefficient $\left(\mathrm{d}_{\text {eff }}\right)$ along the two symmetric directions is not necessarily equivalent unless, as indicated in Ref. [18], one of the contributing nonlinear optical tensor coefficients $\left(d_{12}\right)$ has zero magnitude. By measuring the effective coupling coefficients ( $\left(\mathrm{d}_{\mathrm{eff}}\right)$ for the two symmetric propagation directions in $\mathrm{GdCOB}$ and $\mathrm{YCOB}$, we are able to determine the magnitudes of the two nonlinear optical tensor coefficients, $d_{12}$ and $d_{32}$. As seen in Table 3.1, the absolute values for $\mathrm{d}_{\text {eff }}$ measured at $\pm 70^{\circ}$ in $\mathrm{GdCOB}$ (i.e. for the two symmetric directions) are 0.38 and $0.78 \mathrm{pm} / \mathrm{V}$, respectively. The absolute values for $\mathrm{d}_{\text {eff }}$ measured at $\pm 57^{\circ}$ in $\mathrm{YCOB}$ are 0.69 and $1.12 \mathrm{pm} / \mathrm{V}$, respectively. The value for the larger $/ \mathrm{d}_{\text {eff }}$ for GdCOB is in agreement with the value presented in Ref. [19]. The smaller Ideff $_{\text {value }}$ that we measure for $\mathrm{YCOB}$ is in agreement with the value given in Ref. [18]. However, the fact that the two absolute values for $d_{\text {eff }}$ are significantly different for the two positions in either GdCOB and YCOB contradicts the assertion in Ref. [18] that the contribution from $d_{12}$ is nearly zero.

The two sets of $d_{e f f}$ values for GdCOB and YCOB in Table 3.1 allow us to calculate absolute numeric values for $d_{12}$ and $d_{32}$ using Eqn. (3.1). The absolute values for $d_{12}$ and $d_{32}$ determined for GdCOB and YCOB are shown in Table 3.2. Note that the $d_{12}$ values for GdCOB and YCOB are equal within the experimental uncertainty and likewise for the $\mathrm{d}_{32}$ values.

If the absolute values for $d_{12}$ and $d_{32}$ for YCOB are used in Eqn. (3.1) to calculate the 
Table 3.2. Experimentally determined nonlinear tensor coefficients for GdCOB and YCOB.

\begin{tabular}{|c|c|c|}
\hline Crystal & $\begin{array}{c}\left|\mathrm{d}_{12}\right| \\
(\mathrm{pm} / \mathrm{V})\end{array}$ & $\begin{array}{c}\left|\mathrm{d}_{32}\right| \\
(\mathrm{pm} / \mathrm{V})\end{array}$ \\
\hline GdCOB & $0.22 \pm 0.05$ & $1.72 \pm 0.13$ \\
\hline YCOB & $0.26 \pm 0.04$ & $1.69 \pm 0.17$ \\
\hline
\end{tabular}

maximum $\mid d_{\text {eff }}$ for type I SHG in LaCOB at $1064 \mathrm{~nm}$ (i.e. $|\phi|=80.1^{\circ}$ ), we obtain $\left|d_{\text {eff }}\right|=$ $0.55 \mathrm{pm} / \mathrm{V}$ which is identical within the experimental uncertainty to our measured value. A similar calculation for type I SHG in LaCOB at $1047 \mathrm{~nm}$ (i.e. $|\phi|=84.5^{\circ}$ ) gives a maximum $\mathrm{d}_{\mathrm{eff}}$ of $0.41 \mathrm{pm} / \mathrm{V}$, which also agrees with our experimentally determined value. Therefore from these measurements and calculations, we conclude that the absolute values for the tensor coefficients $d_{12}$ and $d_{32}$ vary very little between GdCOB, YCOB, and LaCOB. Therefore the magnitude for the effective nonlinear coupling $\left(\mid \mathrm{d}_{\mathrm{eff}}\right)$ for any type I SHG interaction in the xz principal plane in any of the three crystals can be determined using the values given for $d_{12}$ and $d_{32}$ in Table 3.2 and Eqn. (3.1).

\subsection{Thermal sensitivities $\left(\beta_{T}\right)$ for SHG}

We studied the variation of type I SHG at $1064 \mathrm{~nm}$ in the $3.75 \mathrm{~mm}$ LaCOB sample over the temperature range of 20 to $100^{\circ} \mathrm{C}$. The data was collected using a sample holder whose temperature could be controlled (increased or decreased). The temperature variable sample holder was attached to the rotation stage in Fig. 3.2. Starting at room temperature, the sample was first tuned in angle to the phasematching direction and then the temperature of the sample was varied while the SHG $(2 \omega)$ irradiance was measured. The data collected for LaCOB is shown in Fig. 3.7. Thermal tuning data was also 
collected on the LBO sample and is shown in Fig. 3.7 for comparison.

The data in Fig. 3.7 for LaCOB reveals that the SHG irradiance was essentially constant from $20-100{ }^{\circ} \mathrm{C}$. The insensitivity of $\mathrm{LaCOB}$ over this broad range of temperatures is remarkable. The thermal insensitivity of LaCOB made it difficult to discern clear $\operatorname{sinc}^{2}$ behavior in the data. We do however show fits of Eqn. (2.52) to both sets of data in Fig. 3.7 (i.e. the solid lines) from which we determine a value for $\beta_{\mathrm{T}}$ of 1.2 $\left(\mathrm{cm}^{-}{ }^{\circ} \mathrm{C}\right)^{-1}$ for LBO and we estimate an upper bound to $\beta_{\mathrm{T}}$ of $0.1\left(\mathrm{~cm}^{-}{ }^{\circ} \mathrm{C}\right)^{-1}$ for $\mathrm{LaCOB}$. Therefore type I LaCOB is at least 10 times less thermally sensitive than type II LBO.

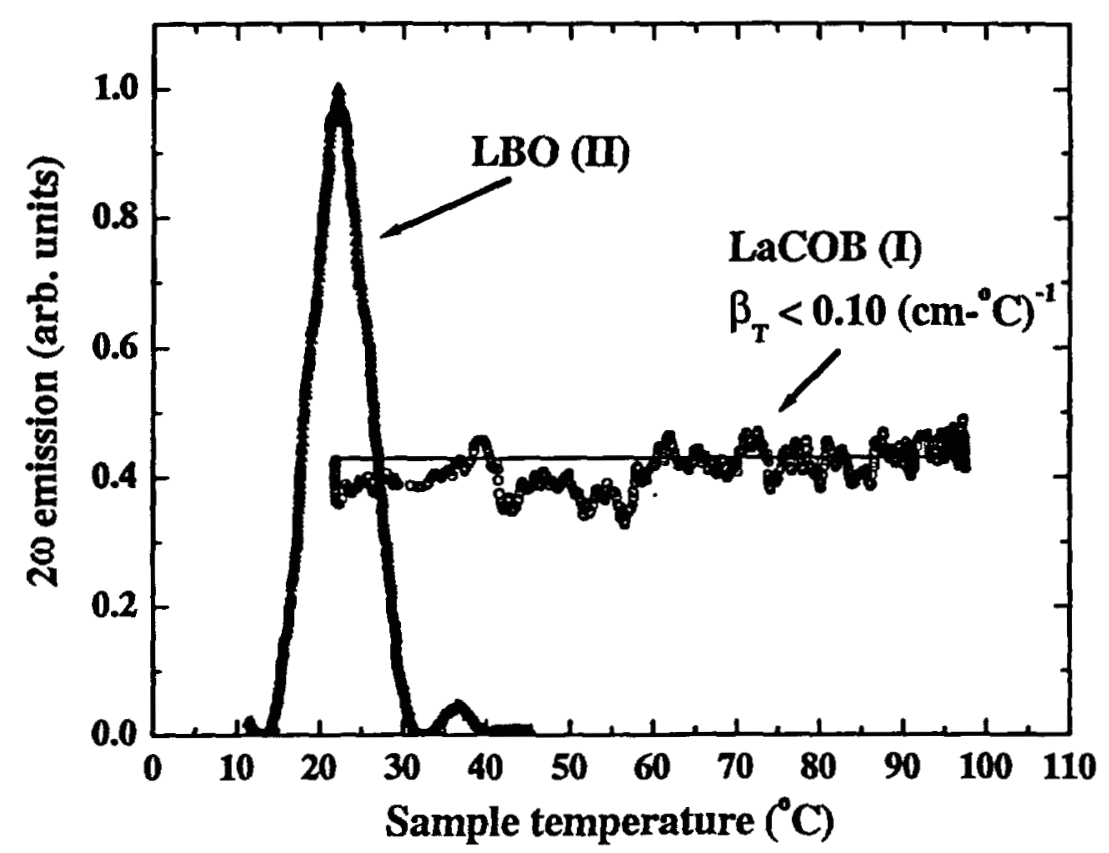

Figure 3.7. Experimentally measured variation of the $2 \omega(532 \mathrm{~nm})$ irradiance with temperature for type I SHG in LaCOB and type II SHG in LBO.

\subsection{Summary}

We have successfully grown the new nonlinear crystal lanthanum calcium oxide borate (LaCOB) and characterized its nonlinear optical properties. A ldeff of $0.52 \pm 0.05$ 
$\mathrm{pm} / \mathrm{V}$ and an angular sensitivity of $1224 \pm 184(\mathrm{~cm}-\mathrm{rad})^{-1}$ for type I SHG at $1064 \mathrm{~nm}$ in LaCOB was determined relative to that of KTP. The $d_{12}$ and $d_{32}$ coefficients of the nonlinear optical tensor for $\mathrm{LaCOB}, \mathrm{GdCOB}$, and $\mathrm{YCOB}$ were determined to have absolute values of $0.26 \pm 0.04 \mathrm{pm} / \mathrm{V}$ and $1.69 \pm 0.17 \mathrm{pm} / \mathrm{V}$, respectively. This implies that the $d_{\text {eff }}$ values for these three materials vary primarily due to differences in their birefringence (i.e. differences in their phasematching angles). From phasematching angle measurements for type I SHG at 1064 and $1047 \mathrm{~nm}$, we predict that LaCOB has a type I non-critically phasematched SHG wavelength of $1042 \pm 1.5 \mathrm{~nm}$. We also determined experimentally that the thermal sensitivity of type I SHG at $1064 \mathrm{~nm}$ in LaCOB is less than $0.1\left(\mathrm{~cm}^{-}{ }^{\circ} \mathrm{C}\right)^{-1}$. Due to its low angular and thermal sensitivities, LaCOB may have potential use in high-average power external and intracavity frequency doubling applications.

We set out to find new crystals with type I NCPM SHG wavelengths near $1050 \mathrm{~nm}$. Our measurements indicate that LaCOB has a type I NCPM SHG wavelength of $1042 \pm$ $1.5 \mathrm{~nm}$. Unfortunately the corresponding coupling coefficient $\left(\mathrm{d}_{12}\right)$ will have a magnitude of only $0.26 \mathrm{pm} / \mathrm{V}$. However, several important uses may exist for the relatively large $d_{32}$ coefficient $(1.69 \mathrm{pm} / \mathrm{V})$ determined for the COB crystals. One such application may be found in a NCPM type II optical parametric amplifier or oscillator. We will investigate this possible use for the COB crystals in the next chapter. Also, if there exists a correspondingly large electro-optic coefficient $\left(r_{23}\right)$, then the COB crystals may also have application as an electro-optic material. We will investigate this possibility in Chapter 5. 


\section{References for Chapter 3}

[1] R. Norrestam, M. Nygren, and J.-O. Bovin, Chem. Mater. 4, 737 (1992)

[2] G. Aka, A. Kahn-Harari, F. Mougel, D. Vivien, F. Salin, P. Coquelin, P. Colin, D. Pelenc, and J. Damelet, J. Opt. Soc. Am. B 14, 2238 (1997)

[3] M. Iwai, T. Kobayashi, H. Furuya, Y. Mori, and T. Sasaki, Jpn. J. Appl. Phys. 36, L276 (1997)

[4] Q. Ye and B. H. T. Chai, J. Cryst. Growth 197, 228 (1999)

[5] C. D. Marshall, R. J. Beach, C. Bibeau, C. A. Ebbers, M. A. Emanuel, E. C. Honea, W. F. Krupke, S. A. Payne, H. T. Powell, K. I. Schaffers, J. A. Skidmore, and S. B. Sutton, Laser Physics 8, 741 (1998)

[6] C. A. Ebbers, Lawrence Livermore National Laboratory, private communication (2000)

[7] F. Mougel, G. Aka, F. Salin, D. Pelenc, B. Ferrand, A. Kahn-Harari, and D. Vivien, Optical Society of America Trends in Optics and Photonics Series 26, 709 (1999)

[8] M. Yoshimura, H. Furuya, I. Yamada, K. Murase, H. Nakao, M. Yamazaki, Y. Mori, and T. Sasaki, Optical Society of America Trends in Optics and Photonics Series 26, 702 (1999)

[9] P. B. W. Burmester, T. Kellner, K. Petermann, G. Huber, R. Uecker, and P. Reiche, Appl. Phys. B 68, 1143 (1999)

[10] B. H. T. Chai, Crystal Photonics, Inc., private communication (1997)

[11] C. A. Ebbers, Lawrence Livermore National Laboratory, private communication (2002)

[12] D. Xue, K. Betzler, H. Hesse, and D. Lammers, Solid State Communications 114, $21(2000)$

[13] R. D. Shannon, Acta Cryst. 32, 751 (1976)

[14] K. I. Schaffers, Lawrence Livermore National Laboratory, private communication (2000)

[15] S. P. Velsko, M. Webb, L. Davis, and C. Huang, IEEE J. Quan. Elec. 27, 2182 (1991)

[16] R. C. Eckardt, H. Masuda, Y. X. Fan, and R. L. Byer, IEEE J. Quan. Elec. 26, $922(1990)$

[17] F. Zernike and J. E. Midwinter, Applied Nonlinear Optics, (John Wiley and Sons, Inc., New York, 1973)

[18] C. Chen, Z. Shao, J. Jiang, J. Wei, J. Lin, J. Wang, N. Ye, J. Lu, B. Wu, M. Jiang, M. Yoshimura, Y. Mori, and T. Sasaki, J. Opt. Soc. Am. B 17, 566 (2000)

[19] G. Aka, A. Kahn-Harari, D. Vivien, J.-M. Benitez, F. Salin, and J. Godard, Eur. J. Solid State Inorg. Chem. 33, 727 (1996) 


\section{Chapter 4}

Investigation of $\mathrm{YCa}_{4} \mathrm{O}\left(\mathrm{BO}_{3}\right)_{3}, \mathrm{GdCa} a_{4} \mathrm{O}\left(\mathrm{BO}_{3}\right)_{3}$, and $\mathrm{LaCa}_{4} \mathrm{O}\left(\mathrm{BO}_{3}\right)_{3}$ for potential use in degenerate non-critically phasematched mid-infrared parametric oscillators

\subsection{Introduction}

Sources of tunable coherent radiation in the $1.5-3.5 \mu \mathrm{m}$ range have wide applications in the areas of remote sensing, LIDAR, and the spectroscopy of organic and inorganic compounds [1]. Optical parametric oscillators (OPO) based on nonlinear crystal media are common devices for generating moderate energy, narrow linewidth, and high beam quality radiation tunable in the $1.3-4.5 \mu \mathrm{m}$ region [2]. Crystals used in midinfrared OPO's generally have nonlinearities $\geq 2 \mathrm{pm} / \mathrm{V}$, good thermal conductivity $(<1.5$ W/mK), high damage thresholds $\left(\sim 10 \mathrm{~J} / \mathrm{cm}^{2}\right)$, low bulk absorption $\left(<0.01 \mathrm{~cm}^{-1}\right)$, are nonhygroscopic, and can be fashioned into lengths $\sim 5 \mathrm{~cm}$. Nonlinear crystals for optical parametric amplifier (OPA) and OPO applications should be robust mechanically in the sense that they can be easily handled and polished and they should readily accept a high quality optical coating. Crystals with low spectral and angular sensitivities are particularly advantageous since in high peak power applications the conversion efficiency can often be improved by using shorter pump pulselengths and focusing the pump beam into the nonlinear crystal. However, in a focused arrangement only crystals with a low angular sensitivity will efficiently convert the pump beam. Temperature insensitivity is also desirable, since in high-peak and high-average power applications even small bulk background absorption causes significant thermal deposition in the nonlinear crystal, 
which can cause thermal de-tuning and fracture the crystal. Especially useful are crystals that are non-critically phasematched (NCPM). NCPM mitigates walk-off effects while having very low angular sensitivity. Crystals which, for pumping at $1064 \mathrm{~nm}$, are NCPM and have signal and idler wavelengths that are degenerate (i.e. $\lambda_{s}=\lambda_{i}=2128 \mathrm{~nm}$ ) could be utilized to convert a high power $1064 \mathrm{~nm}$ pump laser into a high power $2128 \mathrm{~nm}$ laser.

Two nonlinear crystals often employed in OPA and OPO's are $\mathrm{LiNbO}_{3}$ and $\mathrm{LBO}$. $\mathrm{LiNbO}_{3}$ is a uniaxial crystal with a high transparency range $(0.4-5.5 \mu \mathrm{m})$ and an effective coupling coefficient of about $6 \mathrm{pm} / \mathrm{V}$ for degenerate optical parametric generation at $1064 \mathrm{~nm}$. Though $\mathrm{LiNbO}_{3}$ has a large coupling coefficient, a relatively low surface damage threshold of $3 \mathrm{~J} / \mathrm{cm}^{2}$ limits its use in high peak power applications. LBO is well suited for high peak power applications because of its high damage threshold of about $10 \mathrm{~J} / \mathrm{cm}^{2}$ and because its type I NCPM wavelength can be tuned with temperature from $900-1700 \mathrm{~nm}$. LBO however is expensive to grow, somewhat suffers from susceptibility to moisture, and typically has a low temperature acceptance.

In Chapter 4, an exploratory study of the $\mathrm{GdCa}_{4} \mathrm{O}\left(\mathrm{BO}_{3}\right)_{3}$ family of nonlinear crystals was conducted which characterized their type I SHG nonlinear coefficients, angular sensitivities, and thermal sensitivities. They were found to all possess the same value for the $d_{32}(=1.69 \mathrm{pm} / \mathrm{V})$ nonlinear tensor coefficient. This coefficient could also be utilized for type II non-critically phasematched sum frequency generation (SFG) down the $x$ dielectric axis which, combined with their low thermal sensitivity, would imply that these crystals might have utility in a high-average power NCPM OPA or OPO. In addition to being $\mathrm{NCPM}$, one of the materials, $\mathrm{YCa} \mathrm{a}_{4} \mathrm{O}\left(\mathrm{BO}_{3}\right)_{3}(\mathrm{YCOB}), \mathrm{GdCa}_{4} \mathrm{O}\left(\mathrm{BO}_{3}\right)_{3}(\mathrm{GdCOB})$, or $\mathrm{LaCa}_{4} \mathrm{O}\left(\mathrm{BO}_{3}\right)_{3}$ ( $\left.\mathrm{LaCOB}\right)$, might have the appropriate birefringence that gives equal 
(degenerate) signal and idler wavelengths for $1064 \mathrm{~nm}$ pumping.

The work in this chapter explores the possibility of using the COB crystals (YCOB, GdCOB, LaCOB) in a degenerate non-critically phasematched OPO with pump wavelengths in the 800 to $1300 \mathrm{~nm}$ range. In the first part of this chapter, we theoretically evaluate some of the important parameters for an OPO based on YCOB. For example, using Sellmeier equations taken from the literature, we calculate the NCPM signal and idler wavelengths for a YCOB based OPO as a function of pump wavelength. Since it would be ultimately desired to use these materials in a non-critically phasematched degenerate OPO (i.e. equal signal and idler wavelengths), the threshold for operation at the calculated degenerate wavelength is then estimated. The required threshold value is a good indication as to the practicality of such a device.

In the second part of this chapter, we describe our experimental technique to measure the NCPM degenerate wavelengths for an OPO based on YCOB. We will use SHG to determine the NCPM degenerate OPO wavelengths. The degenerate OPO process is $\omega_{\text {beo pump }} \rightarrow \omega_{\text {signal }}+\omega_{\text {idler }}=2 \omega_{\text {signal, }}$ since at degeneracy $\omega_{\text {signal }}=\omega_{\text {idler, }}$ and note that this process in reverse is $2 \omega_{\text {signal }} \rightarrow \omega_{\text {opo pump }}$ which can be identified as SHG for a fundamental wavelength of $\omega_{\text {signal. }}$. Therefore by performing an SHG experiment that determines the NCPM SHG wavelength (i.e. where $\omega_{\mathrm{SHG}}=2 \omega_{\mathrm{signal}}$ ) we will determine the NCPM degenerate OPO wavelength. The SHG experiments utilized the signal from a tunable $\mathrm{LiNbO}_{3} \mathrm{OPO}$ as a pump source. The tuning range of the $\mathrm{LiNbO}_{3} \mathrm{OPO}$ was constrained by the available mirrors that were used to construct it and so, consequently, the SHG experiments on YCOB were conducted only over a limited range (1680 - 1900 nm) of generated wavelengths. Encouraging preliminary results from the SHG 
experiments on $\mathrm{YCOB}, \mathrm{LaCOB}$, and $\mathrm{GdCOB}$ are presented and suggest refinement of the experiment and continued measurements.

4.2 Theoretical estimates of the type II NCPM OPO wavelengths, OPO threshold, and SHG efficiency and wavelength sensitivity for $\mathrm{YCOB}$

\subsubsection{Non-critical OPO phasematching conditions}

The nonlinear process will only be efficient if the process is phasematched. As discussed in Chapter 2, section 2.4.2, phasematching can be described as conservation of momentum. Eqns. (4.1) and (4.2) express, mathematically, the conditions for conservation of energy and momentum (i.e. $\Delta k=0$ ) for a type II OPO process down the $x$ dielectric axis in a negative biaxial (YCOB) crystal. In Eqns. (4.1) and (4.2), $\lambda_{p}, \lambda_{s}$, and

$$
\begin{gathered}
\frac{1}{\lambda_{p}}=\frac{1}{\lambda_{s}}+\frac{1}{\lambda_{i}} \\
\Delta k=2 \pi\left(\frac{n_{y}\left(\lambda_{p}\right)}{\lambda_{p}}-\frac{n_{y}\left(\lambda_{s}\right)}{\lambda_{s}}-\frac{n_{z}\left(\lambda_{i}\right)}{\lambda_{i}}\right)=0
\end{gathered}
$$

$\lambda_{i}$ are the pump, signal, and idler wavelengths, respectively and $n_{y}$ and $n_{z}$ are the principal refractive indices at the wavelengths specified in the $y$ and $z$ directions, respectively. The wavelengths that satisfy Eqns. (4.1) and (4.2) obviously depend on the values of the refractive indices of the crystal. The refractive indices for YCOB and GdCOB have been determined by Ref. [3] in terms of Sellmeier equations. Therefore in section 4.2.3 using Sellmeier equations for YCOB, we will look for wavelengths that satisfy Eqns. (4.1) and (4.2). 


\subsubsection{Sellmeier equations}

As was stated in the last section, Sellmeier equations will be used in the estimate of the type II non-critically phasematched OPO wavelengths (i.e. signal and idler for pump wavelengths between $800-1300 \mathrm{~nm}$ ) for propagation along the $\mathrm{x}$ dielectric axis in YCOB. Sellmeier equations for YCOB were derived by Ref. [3] and are given in Eqns. (4.3) - (4.5). The wavelength values used in Eqns. (4.3) - (4.5) are to be expressed in

$$
\begin{aligned}
& \mathrm{n}_{\mathrm{x}}=\left(2.81697+\frac{.022418}{\lambda^{2}-.0149216}-7.73709 \times 10^{-5} \lambda^{2}\right)^{\frac{1}{2}} \\
& \mathrm{n}_{\mathrm{y}}=\left(2.91924+\frac{.0252847}{\lambda^{2}-.0130221}-6.44650 \times 10^{-5} \lambda^{2}\right)^{\frac{1}{2}} \\
& \mathrm{n}_{\mathrm{z}}=\left(2.95452+\frac{.025835}{\lambda^{2}-.0125276}-7.64814 \times 10^{-5} \lambda^{2}\right)^{\frac{1}{2}}
\end{aligned}
$$

microns ( $\mu \mathrm{m})$. If the phasematched wavelengths for YCOB resulting from calculations using these Sellmeier equations are $>1.5 \mu \mathrm{m}$, they should only be regarded as rough estimates since these Sellmeier equations were experimentally derived by Ref. [3] using wavelengths $\leq 1.3 \mu \mathrm{m}$.

\subsubsection{Non-critically phasematched wavelengths}

The signal and idler wavelengths satisfying $\Delta \mathrm{k}=0$ for pump wavelengths in the range $800-1300 \mathrm{~nm}$ were calculated with standard spreadsheet software using Eqns. (4.1), (4.2), (4.4), and (4.5). Fig. 4.1 shows the result of the calculation for YCOB. The calculation yields phasematched signal and idler wavelengths of $2.08 \mu \mathrm{m}$ and $2.178 \mu \mathrm{m}$, 
respectively, for a pump wavelength of $1.064 \mu \mathrm{m}$. Also, the values for the degenerate wavelengths can be determined from the crossing point of the curves in Fig. 4.1. The estimated degenerate signal and idler wavelengths are $\lambda_{s}=\lambda_{i}=2100 \mathrm{~nm}$ and $\lambda_{p}=\lambda_{s} / 2=$ $1050 \mathrm{~nm}$.

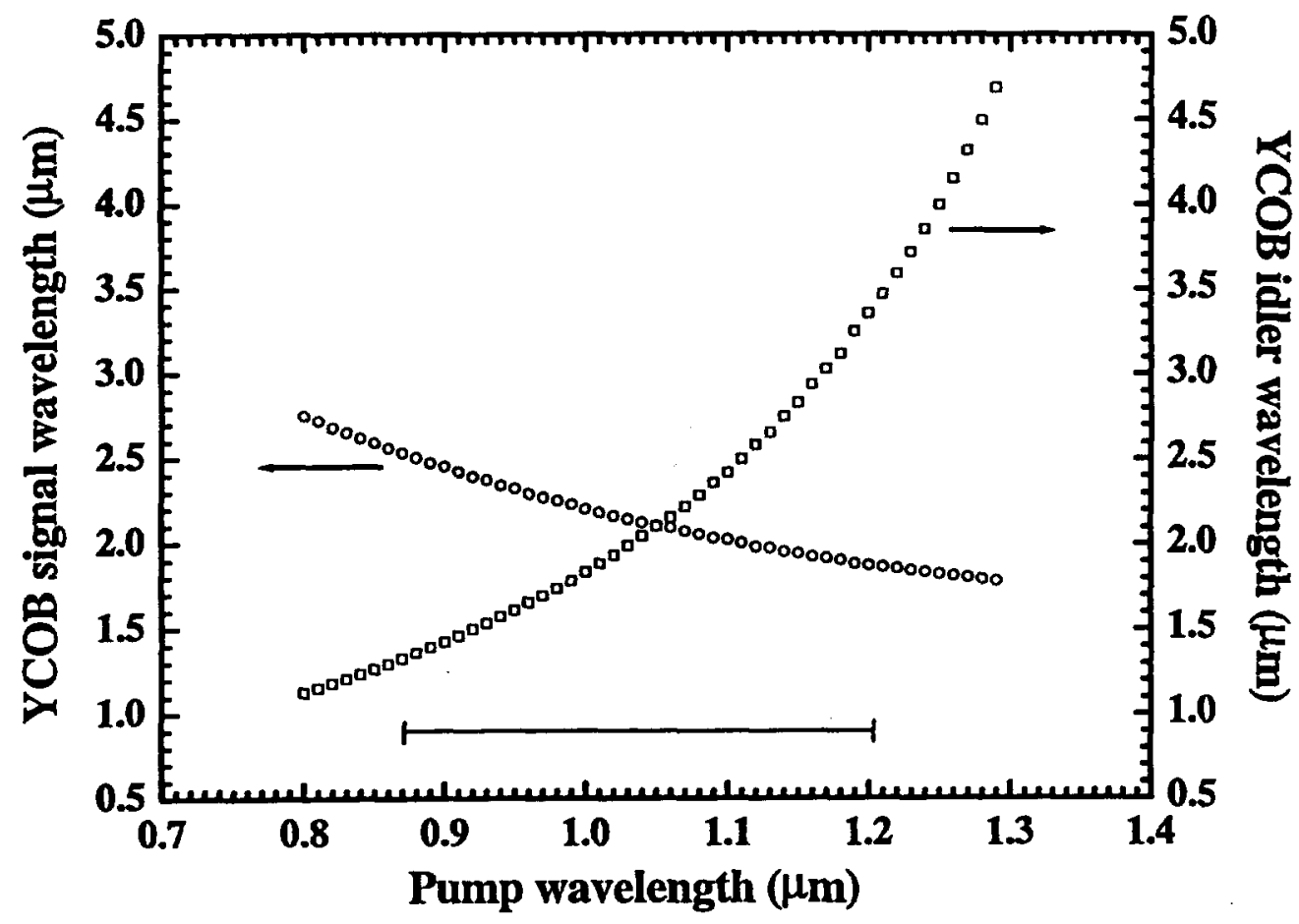

Figure 4.1. Calculated signal and idler wavelengths for type II SFG for pumping down the $x$ dielectric axis in YCOB. The error bar shows the uncertainty to the degenerate point for an average uncertainty to the $\ln _{z}-n_{y} \mid$ birefringence of about 0.004 .

We would like to estimate the uncertainty in the wavelength of the degenerate point in Fig. 4.1 since we don't expect the Sellmeier equations to be very accurate in this wavelength region. An estimate of the uncertainty to the degenerate wavelength will give us an idea of the possible tuning range that will be needed from our pump laser for the SHG experiments. Therefore to estimate this uncertainty, the variation of the degenerate point with differences in the $\ln _{z}-n_{y} \mid$ birefringence (i.e. $\Delta(\Delta n)$ ) in the Sellmeier equations 
was calculated. It was found that for an increase and decrease in the $\ln _{z}-n_{y} \mid$ birefringence in the Sellmeier equations of +0.005 and -0.003 , respectively, the degenerate point (i.e. the crossing point in Fig. 4.1) shifted to pump wavelengths of $0.87 \mu \mathrm{m}$ and $1.21 \mu \mathrm{m}$, respectively. The error bar on Fig. 4.1 represents this total shift. An uncertainty of about 0.004 to the birefringence in the Sellmeier equations is not that unreasonable because when Sellmeier equations for YCOB from different researchers [3-4] are compared it is found that the difference in their $\left|n_{z}-n_{y}\right|$ birefringence at $2.1 \mu \mathrm{m}$ is about 0.003 . Therefore, the pump source for our SHG experiments should ideally have a tuning range from about $1.7-2.4 \mu \mathrm{m}$.

\subsubsection{Degenerate OPO threshold estimate}

A strong indicator of the feasibility of utilizing YCOB in a degenerate $-2 \mu \mathrm{m}$ OPO would be the value of the OPO threshold necessary to obtain oscillation from this material. The pump irradiance required to reach oscillation threshold in a continuouswave degenerate singly resonant oscillator (SRO) can be calculated using Eqn. (2.91). Eqn. (2.91) is for the case of plane waves, perfect phasematching, and an un-depleted continuous-wave pump. Using the following parameter values,

$\mathrm{n}=$ refractive index for YCOB (values for the signal, idler, and pump wavelength are assumed to be equal) $=1.7$

$\mathrm{c}=3 \times 10^{10} \mathrm{~cm} / \mathrm{s}$

$\lambda_{1}=\lambda_{i}=\lambda_{2}=\lambda_{s}=2.1 \times 10^{-4} \mathrm{~cm}$

$R=$ reflectivity of the output coupler at the signal wavelength $=0.98$

$\mathrm{d}=\mathrm{d}_{32}=4.034 \times 10^{-9} \mathrm{esu}=1.69 \mathrm{pm} / \mathrm{V}$

$\mathrm{L}=1.78 \mathrm{~cm}$

the oscillation threshold for a YCOB OPO is estimated to be $3.22 \mathrm{MW} / \mathrm{cm}^{2}$. A similar calculation for a $1.78 \mathrm{~cm} \mathrm{LiNbO} 3$ crystal where $\mathrm{d}=6 \mathrm{pm} / \mathrm{V}$ and $\mathrm{n}=2.2$ yields a threshold 
of $580 \mathrm{KW} / \mathrm{cm}^{2}$. Note the threshold value for the $\mathrm{LiNbO}_{3}$ crystal is about 6 times less than for YCOB. The lower threshold for $\mathrm{LiNbO}_{3}$ is due to its larger coupling coefficient (d).

It is also desirable to estimate the threshold for a pulsed OPO based on YCOB since relatively high pump intensities can be achieved with Q-switched lasers. The threshold fluence for a degenerate pulse-pumped $\mathrm{YCOB}$ OPO can be calculated using the expression developed by Brosnan and Byer [5] given in Eqn. (2.92). Using the following parameter values,

$\tau=1 / \mathrm{e}^{2}$ temporal pump pulselength $=10 \mathrm{~ns}$ $\kappa=$ nonlinear coupling constant for YCOB $=\frac{8 \pi^{2} d_{\text {eff }}^{2}}{\lambda_{s} \lambda_{i} n_{s} n_{i} n_{p} \varepsilon_{o} c}$ $g_{s}=$ signal spatial mode coupling coefficient $=\frac{\omega_{p}^{2}}{\omega_{p}^{2}+\omega_{s}^{2}}=\left(\right.$ for $\omega_{p}=\omega_{s}=$ pump or signal beam spot sizes $=2 \mathrm{~mm}$ ) $=0.5$

$L=$ effective parametric gain length $=$ YCOB crystal length $=1.78 \mathrm{~cm}$ $L=$ optical cavity length $=L^{\prime}($ physical cavity length $)+(n-1) \ell($ physical crystal length) $\frac{P_{n}}{P_{0}}=\frac{\text { signal threshold power }}{\text { signal noise power }} \equiv 10^{14}$ (determined in general by Brosnan and Byer) $\alpha=$ YCOB crystal absorption coefficient @ $2100 \mathrm{~nm}=0.067 \mathrm{~cm}^{-1}$

$\ell=$ YCOB crystal length $=1.78 \mathrm{~cm}$

$\mathrm{R}=$ reflectivity of the output coupler @ $2100 \mathrm{~nm}=0.98$

$\mathrm{d}_{\text {eff }}=\mathrm{d}_{32}=1.69 \times 10^{-12} \mathrm{~m} / \mathrm{V}$

$\lambda_{\mathrm{s}}=\lambda_{\mathrm{i}}=2100 \times 10^{-9} \mathrm{~m}$

$\mathrm{n}_{\mathrm{s}}=\mathrm{n}_{\mathrm{i}}=\mathrm{n}_{\mathrm{p}}=\mathrm{n}=1.7$

$\varepsilon_{0}=8.8542 \times 10^{-12} \mathrm{C}^{2} \mathrm{~s}^{2} / \mathrm{kg} \mathrm{m}^{3}$

$\mathrm{c}=3 \times 10^{8} \mathrm{~m} / \mathrm{s}$

$\mathrm{L}^{\prime}=8 \mathrm{~cm}$

an estimate for the threshold fluence is calculated to be $8.07 \mathrm{~J} / \mathrm{cm}^{2}$. For comparison, the threshold pump fluence for a $\mathrm{LiNbO}_{3}$ OPO operating at degeneracy (i.e. $\lambda_{\mathrm{s}}=2128 \mathrm{~nm}$ ) using a $1.78 \mathrm{~cm}$ long crystal is calculated with Eqn. (2.92) to be $1.08 \mathrm{~J} / \mathrm{cm}^{2}$. The $\mathrm{LiNbO}_{3}$ 
OPO threshold is about 8 times lower than that for $\mathrm{YCOB}$ owing to the much larger $\mathrm{d}_{\text {eff }}$ and lower background absorption for $\mathrm{LiNbO}_{3}$.

The threshold fluence of $8.07 \mathrm{~J} / \mathrm{cm}^{2}$ for YCOB corresponds to an irradiance of about $800 \mathrm{MW} / \mathrm{cm}^{2}$ (i.e. using $10 \mathrm{~ns}$ pulses). This value for the required pump irradiance is readily obtainable with a Q-switched laser. The damage threshold for YCOB was estimated experimentally at $1064 \mathrm{~nm}$ using $10 \mathrm{~ns}$ pulses [6] to be about $30 \mathrm{~J} / \mathrm{cm}^{2}$. This is well above the calculated threshold fluence of $8.07 \mathrm{~J} / \mathrm{cm}^{2}$ which implies that threshold could be achieved without damaging the YCOB crystal.

\subsubsection{SHG efficiency estimate}

SHG experiments were planned for the COB crystals to precisely evaluate their degenerate OPO pump wavelengths. Before beginning the experiments it makes logical sense to predict the level of the SHG signal that would be produced by the COB crystals so that we can determine a priori if the signal will be detectable and what type of detector we will need. An efficiency estimate for SHG for perfect phasematching in YCOB down the $\mathrm{x}$-axis was calculated using Eqn. (2.54) written in MKS units as,

$$
\eta_{\text {eff }}=\frac{I_{2 \omega}}{I_{1 \omega}}=\frac{2 \pi^{2} d_{\text {eff }}^{2} L^{2} I_{1 \omega}}{\lambda_{2 \omega}^{2} n_{1 \omega}^{2} n_{2 \omega} \varepsilon_{0} c}
$$

where,

$I_{2 \omega}=$ generated SHG irradiance at $1050 \mathrm{~nm}$

$I_{1 \omega}=1 \omega$ pump power at $2100 \mathrm{~nm}-0.5 \mathrm{~mJ} /\left((4 \mathrm{~ns})\left(\pi(2.0 \mathrm{~mm})^{2}\right)\right)$ (typical irradiance from a $\mathrm{LiNbO}_{3}$ OPO) $=9.94 \mathrm{GW} / \mathrm{m}^{2}$

$\mathrm{d}_{\text {eff }}=\mathrm{d}_{32}=1.69 \times 10^{-12} \mathrm{~m} / \mathrm{V}$

$\mathrm{L}=$ crystal length $=0.0178 \mathrm{~m}$

$\varepsilon_{0}=8.8542 \times 10^{-12} \mathrm{C}^{2} \mathrm{~s}^{2} / \mathrm{kg} \mathrm{m}^{3}$ 
$\mathrm{c}=3 \times 10^{8} \mathrm{~m} / \mathrm{s}$

$\mathrm{n}_{1 \omega}=\mathrm{n}_{2 \omega}=1.7$

$\lambda_{2 \omega}=1050 \times 10^{-9} \mathrm{~m}$

inserted into Eqn. (4.6) gives $\eta_{\text {eff }}=0.0125$. This implies about a $1.3 \%$ efficiency for SHG. A $2 \omega$ energy of $6.25 \mu \mathrm{J}$ can be calculated for this $1.3 \%$ efficiency from $0.5 \mathrm{~mJ}$ of $1 \omega$ pump energy. This level of SHG energy was encouraging experimentally because it can easily be measured with a Si detector.

Thus given the results of these calculations, the decision was made to determine if the $1.6-2.4 \mu \mathrm{m}$ range contained the type II non-critically phasematched SHG wavelength for YCOB.

\subsubsection{Wavelength sensitivity estimates for $\mathrm{YCOB}$ and $\mathrm{LiNbO}_{3}$}

Our SHG experiments will involve measuring the SHG response of the crystals as a function of the pump wavelength. It would be interesting to know what to expect for the wavelength sensitivity $\left(\beta_{\lambda}\right)$ for the COB crystals since this quantity should be directly observable from the experimental data. We can calculate $\beta_{\lambda}$ using the Sellmeier equations given in Eqns. (4.3) - (4.5) and Eqn. (2.72) specified with respect to the pump wavelength. The expression for $\Delta k$ used to calculate $\beta_{\lambda}$ will be that which describes type II SHG in the xy principal plane in YCOB. Eqn. (4.7) describes $\triangle k$ as a function of refractive indices and wavelength for this process as,

$$
\Delta k=\frac{2 \pi}{\lambda_{1 \omega}}\left(2 n_{x y}\left(\lambda_{2 \omega}\right)-n_{z}\left(\lambda_{1 \omega}\right)-n_{x y}\left(\lambda_{1 \omega}\right)\right)
$$

where $n_{x y}(\lambda)$ is given by, 


$$
n_{x y}(\lambda)=\frac{n_{x}(\lambda) n_{y}(\lambda)}{\left(\left(n_{x}(\lambda) \sin \theta\right)^{2}+\left(n_{y}(\lambda) \cos \theta\right)^{2}\right)^{\frac{1}{2}}}
$$

with $\theta$ being measured from the y dielectric axis. We will choose to calculate $\beta_{\lambda}$ at 1725 $\mathrm{nm}$ for two reasons. First, because this wavelength is within the range of degenerate wavelengths for YCOB that we previously calculated, and secondly, because it is on the short wavelength side of the range which is where the Sellmeier equations are probably most accurate. The wavelength sensitivity, defined by Eqn. (2.72), can be seen as the slope of $\Delta \mathrm{k}$ as a function of $\lambda_{1 \omega}$. We will start by setting $\Delta \mathrm{k}$ equal to zero at $1725 \mathrm{~nm}$ and calculating values for $\Delta k$ for wavelength increments of $\pm 50 \mathrm{~nm}$ about $1725 \mathrm{~nm}$ (i.e calculating $\Delta k$ at $1675 \mathrm{~nm}$ and at $1775 \mathrm{~nm})$. $\beta_{\lambda}$ will then be calculated using $(\Delta \mathrm{k}(1775$ $\mathrm{nm})-\Delta \mathrm{k}(1675 \mathrm{~nm})) / 100 \mathrm{~nm}$. The result of the calculation yields a value for $\beta_{\lambda}$ of 0.82 $(\mathbf{c m}-\mathbf{n m})^{-1}$ for YCOB. We would like to note two things about this calculation. First, the choice that $\Delta \mathrm{k}=0$ at $1725 \mathrm{~nm}$ implies that

$$
2 n_{y}(862.5 n m)=\left(n_{z}(1725 n m)+n_{y}(1725 n m)\right)=3.4367348
$$

This value is assumed for both $1 \omega$ terms that occur in the calculation for $\Delta \mathrm{k}$ on either side of $\Delta \mathrm{k}=0$. This is a reasonable assumption since there is no a priori reason that the difference between $n_{y}$ and $n_{z}$ for wavelengths in the range $1600-1800 \mathrm{~nm}$ would be anything but relatively constant. Secondly, the wavelength range of $100 \mathrm{~nm}$, over which $\beta_{\lambda}$ was calculated, was chosen arbitrarily, however as a check, the range was decreased to $10 \mathrm{~nm}$ and a less than $10 \%$ change in $\beta_{\lambda}$ was seen.

As a comparison to our calculated value for $\beta_{\lambda}$ for $\mathrm{YCOB}$ and for reference for our 
coming experiments, $\beta_{\lambda}$ was calculated for $\mathrm{LiNbO}_{3}$ in a similar manner. A value for $\beta_{\lambda}$ of $6.63(\mathrm{~cm}-\mathrm{nm})^{-1}$ was calculated for $\mathrm{LiNbO}_{3}$ for the case of type I SHG in the yz principal plane for phasematching at $1750 \mathrm{~nm}$. This value for $\mathrm{LiNbO}_{3}$ is about 8 times that for YCOB. Therefore, according to Eqn. (2.73), we expect the SHG response for our YCOB crystal to have about 20 times the width in wavelength as that for the $\mathrm{LiNbO}_{3}$ crystal.

\subsection{Type II non-critically phasematched SHG experiments}

\subsubsection{General description of the experimental procedure}

From the phasematched wavelengths shown in Fig. 4.1, we expect the COB crystals to have phasematched fundamental SHG wavelengths somewhere between 1.7 and 2.4 $\mu \mathrm{m}$. In order to measure the phasematched SHG wavelengths, we need a tunable source that emits in this wavelength range. $\mathrm{A} \mathrm{LiNbO}_{3} \mathrm{OPO}$ pumped by a Q-switched Nd:YAG laser can provide tunable output from approximately $1.4-4.0 \mu \mathrm{m}$. Tuning the $\mathrm{LiNbO}_{3}$ OPO is usually accomplished by varying the angle of the $\mathrm{LiNbO}_{3}$ crystal in the OPO cavity. Therefore, we will first setup a $\mathrm{LiNbO}_{3}$ OPO pumped by a commercial $\mathrm{Nd:YAG}$

-1aser and characterize its tuning range. SHG experiments will then be conducted down the $\mathrm{x}$ dielectric axis of the $\mathrm{COB}$ crystals using the tunable signal from the $\mathrm{LiNbO}_{3} \mathrm{OPO}$. Fig. 4.2 shows a schematic of the $\mathrm{SHG}$ experiment utilizing the tunable $\mathrm{LiNbO}_{3} \mathrm{OPO}$ and a COB crystal to illustrate our basic experimental technique. 


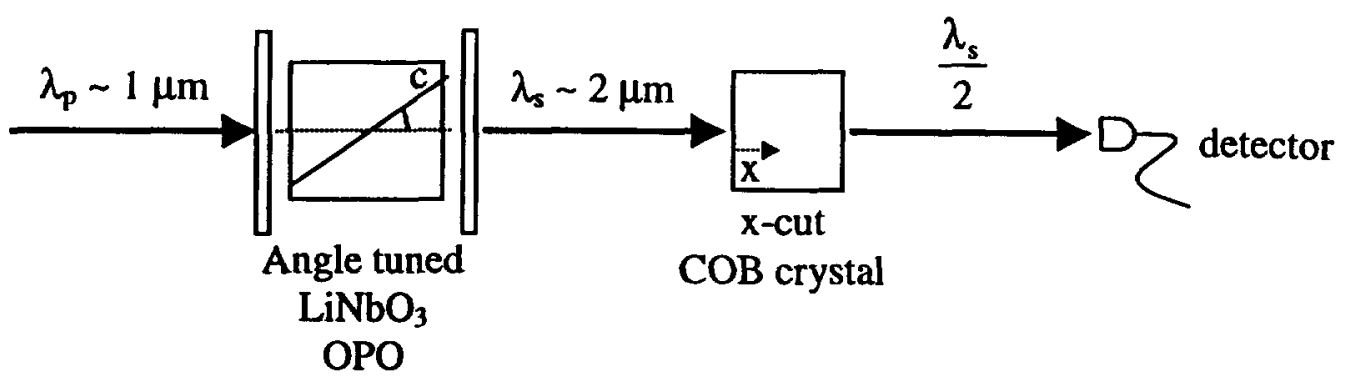

Figure 4.2. Schematic diagram of the experiment to measure the non-critically phasematched SHG wavelengths for propagation along the $\mathrm{x}$ dielectric axis in the $\mathrm{COB}$ crystals.

\subsubsection{Construction and characterization of a tunable $\mathrm{LiNbO}_{3} \mathrm{OPO}$}

The first part of the experimental work was to construct, successfully operate, and characterize a tunable $\mathrm{LiNbO}_{3} \mathrm{OPO}$. A diagram of the $\mathrm{LiNbO}_{3} \mathrm{OPO}$ and its pump laser is shown Fig. 4.3. The actual $\mathrm{LiNbO}_{3} \mathrm{OPO}$ was constructed using two flat $\mathrm{CaF}_{2}$ mirrors (as can be seen in Fig. 4.3) that were obtained in the laboratory from a decommissioned experiment. The input coupler (IC) to the OPO was $90 \%$ transmissive at $1.064 \mu \mathrm{m}$ and $40-60 \%$ transmissive from $1.5-2.0 \mu \mathrm{m}$. The high reflector (HR) was $95 \%$ transmissive at $1.064 \mu \mathrm{m}$ and $20-100 \%$ transmissive from $1.5-2.0 \mu \mathrm{m}$. The $\mathrm{LiNbO}_{3}$ OPO was tuned by varying the angle of the crystal within the cavity using a Klinger stepper-motor driven rotation stage. The $1200 \mathrm{~mm}$ image relay telescope that follows the Nd:YAG pump laser imaged (i.e. a magnification of 1) the output coupler of the Nd:YAG pump laser onto the $\mathrm{LiNbO}_{3}$ crystal in the OPO. A benefit of relay imaging the Nd:YAG pump beam onto the OPO crystal was that beam arrived approximately collimated at the crystal and that helped boost the output efficiency of the OPO. The OPO output through the input coupler was used as the pump beam for the SHG experiments involving the COB crystals and the OPO output through the high reflector was directed through a 


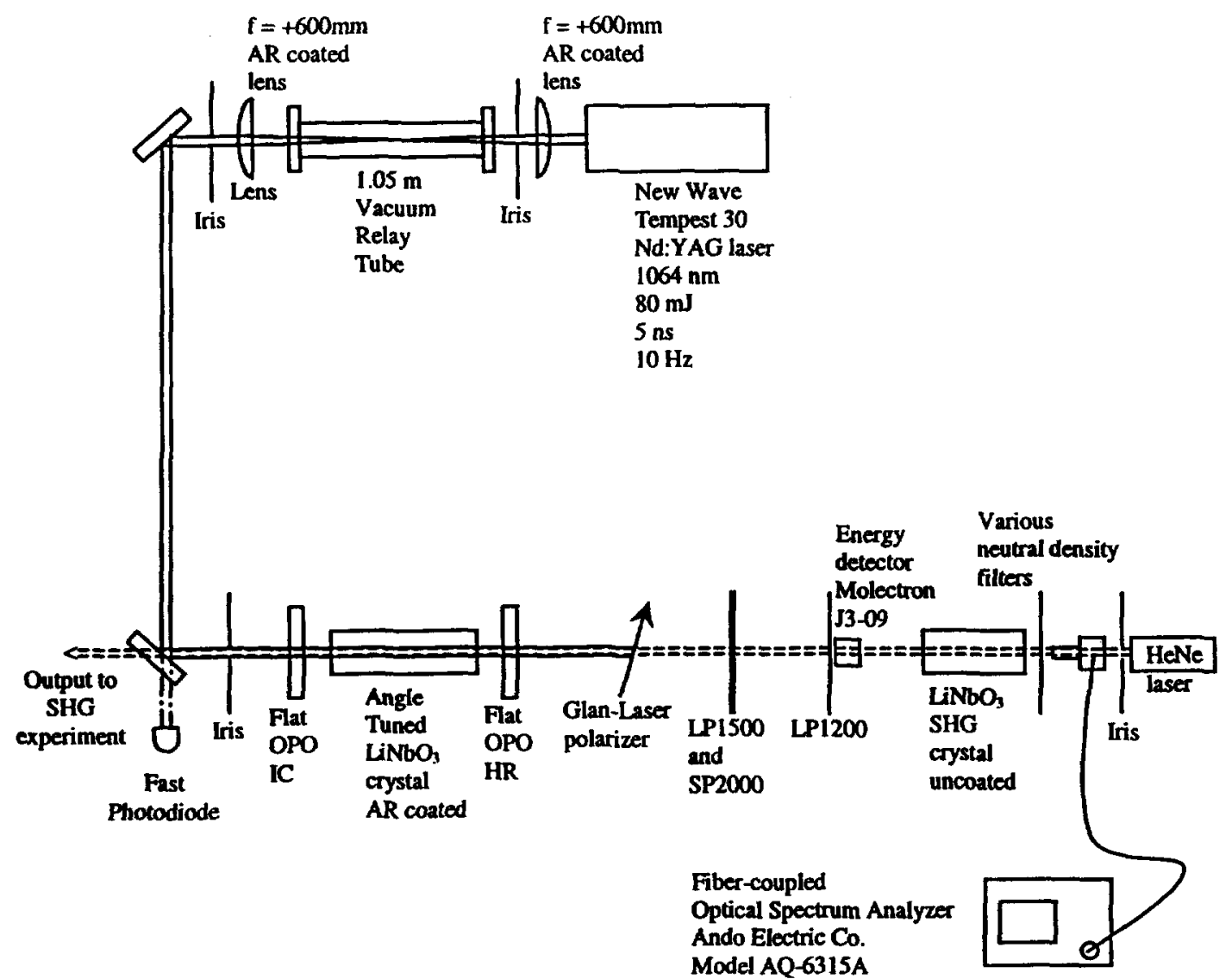

Figure 4.3. $\mathrm{LiNbO}_{3}$ OPO setup. The J3-09 energy meter and LP1200 filter were only placed in the beam path temporarily to measure the OPO output energy at each wavelength setting. The HeNe laser was used to align the OPO optics and crystal.

second $\mathrm{LiNbO}_{3}$ crystal which produced an SHG signal that was used to monitor the OPO output wavelength. The Glan-Laser polarizer separated the $1.064 \mu \mathrm{m}$ pump beam from the OPO signal beam along this direction of the setup.

During the initial construction and operation of the $\mathrm{LiNbO}_{3} \mathrm{OPO}$, some difficulty in obtaining output energies above the $\mu \mathrm{J}$ range was encountered. Efficient conversion requires high irradiance and good pump beam quality. Therefore an $\mathrm{M}^{2}$ analysis was performed on the Nd:YAG pump beam in both the vertical and horizontal directions to determine how close the beam was to a TEM $\mathrm{TH}_{00}$ mode. A beam that is solely $\mathrm{TEM}_{00}$ mode has perfect beam quality (i.e an $\mathrm{M}^{2}=1$ ). Beams which have higher-order mode content 
have $\mathbf{M}^{2}>1$. The $\mathbf{M}^{2}$ factor [7] characterizes how a real beam differs in far-field divergence angle from that of a perfect TEM $_{00}$ beam if both beams have the same waist size. The $M^{2}$ value appears in the expression describing the beam spot size as a function of propagation distance as shown in Eqn. (4.10). In Eqn. (4.10), w(z) is the $1 / \mathrm{e}^{2}$ beam

$$
w(z)=w_{0}^{2}+\left(\frac{M^{2} \lambda}{\pi w_{0}}\right)^{2}\left(z-z_{0}\right)^{2}
$$

spot size (radius) as a function of position along the beam, $w_{0}$ is the beam waist, $z_{0}$ is the $\mathrm{z}$ position of the beam waist, and $\mathrm{M}^{2}$ is the beam quality factor $(\geq 1)$. The Nd:YAG beam was put through a focus using a lens and 21 beam images were collected for positions passing through the focus with a Cohu CCD camera Model ER5001B and a Spiricon Beam Analyzer LBA-100A. The translational position (z) corresponding to each beam image was also recorded. The $1 / \mathrm{e}^{2}$ irradiance widths (spot sizes) in the horizontal $(\mathrm{x})$ and the vertical ( $y$ ) directions for the beam at each $\mathrm{z}$ position were calculated from the images using a spreadsheet program written in Visual Basic. The values for $w_{0}, z_{0}$, and $M^{2}$ in each direction were obtained from a three parameter numerical fit of Eqn. (4.10) to each set of spot sizes. Fig. 4.4 shows the data for the beam spot sizes and the corresponding fit of Eqn. (4.10) in each direction. As can be seen from Fig. 4.4, the Nd:YAG beam has $\mathrm{M}^{2}$ values of 2.206 in the $x$ direction (horizontal) and 2.081 in the $y$ direction (vertical). These values for $\mathrm{M}^{2}$ imply that the beam is not a pure TEM ${ }_{00}$ mode but that it has some higher order mode content. Though the Nd:YAG pump beam did not have an $\mathrm{M}^{2}=1$, it was of good enough quality that it did not end up severely degrading the performance of the $\mathrm{LiNbO}_{3} \mathrm{OPO}$ or cause major damage to the mirrors or crystal. 


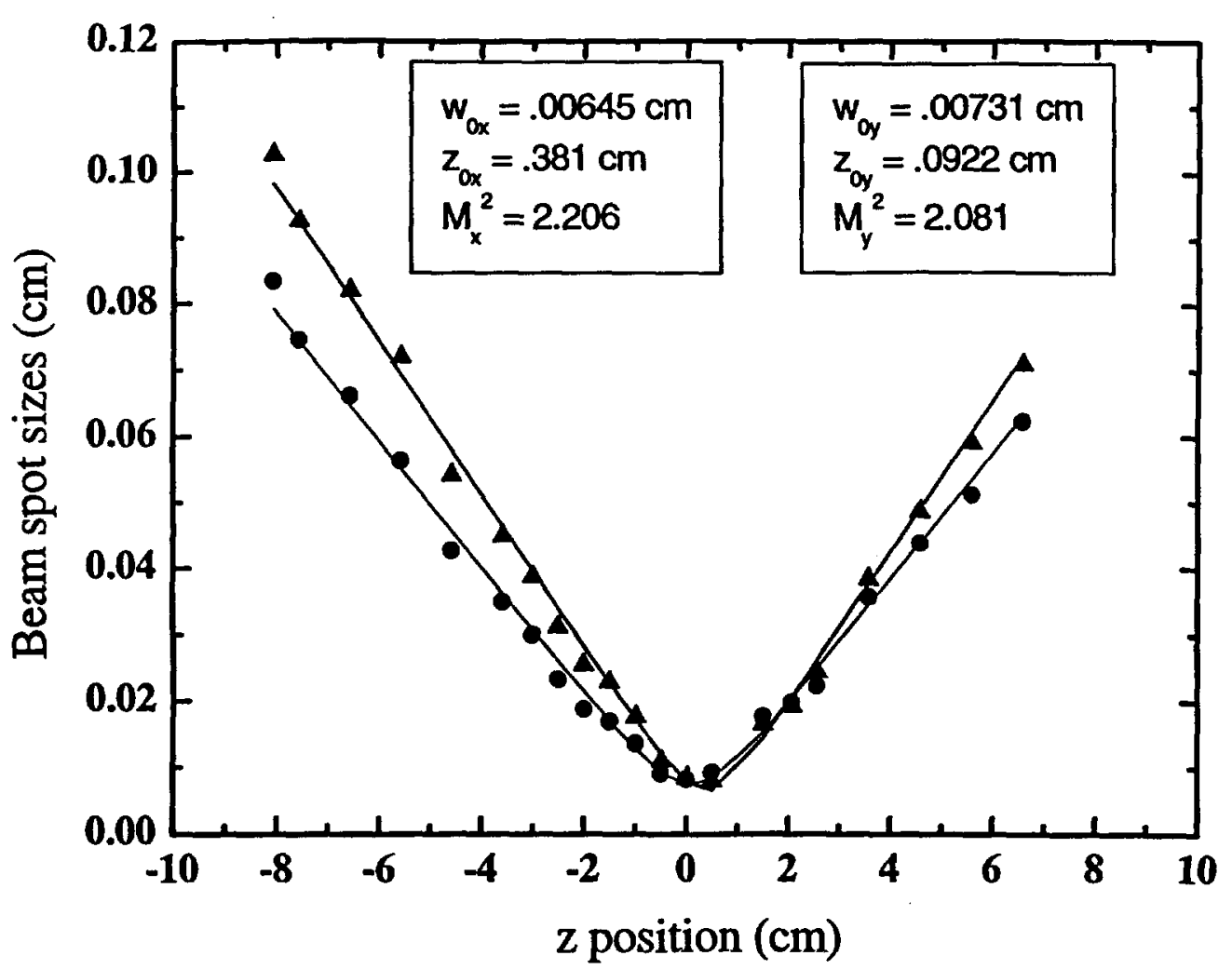

Figure 4.4. Nd:YAG beam $1 / \mathrm{e}^{2}$ irradiance spot sizes vs. position along the beam. The points are the widths calculated from beam images and the lines through the points are fits of Eqn. (4.10) to the data. The insets show the results from the three parameter numerical fit for the beam waist sizes, the positions of the waists, and the $M^{2}$ values for the two transverse directions.

The $\mathrm{LiNbO}_{3}$ OPO signal was tuned in wavelength by changing the angle of the $\mathrm{LiNbO}_{3}$ crystal in the OPO cavity [8]. Fig. 4.5 shows the measured OPO output wavelength vs. change in the external angle of the $\mathrm{LiNbO}_{3}$ crystal. The OPO was tuned by changing the external angle of the crystal in increments of 0.05 degrees using a stepper-motor controlled rotation stage. The $\mathrm{LiNbO}_{3} \mathrm{OPO}$ produced a signal beam that could be tuned in wavelength from about 1625 to $1975 \mathrm{~nm}$.

Fig. 4.6 shows the output energy of the OPO as it is tuned through about the same range of wavelengths. As shown in Fig. 4.6, the output energy of the OPO at about 1650 $\mathrm{nm}$ and $1950 \mathrm{~nm}$ was $<0.10 \mathrm{~mJ} / \mathrm{pulse}$. The drop in output energy below $1650 \mathrm{~nm}$ and 


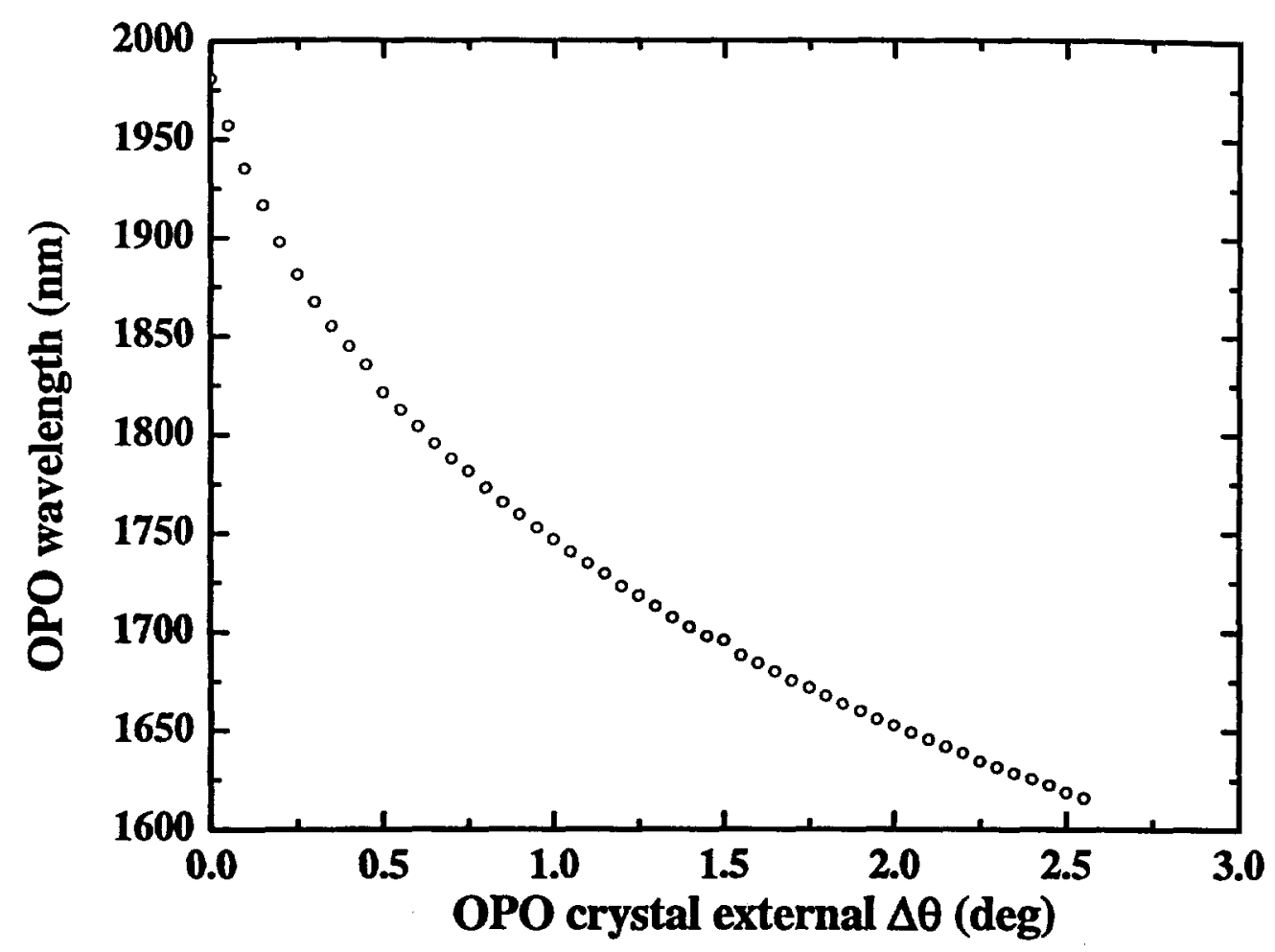

Figure 4.5. Measured $\mathrm{LiNbO}_{3} \mathrm{OPO}$ output wavelength vs. change in external angle of the $\mathrm{LiNbO}_{3}$ crystal. During the experiments, the wavelength of the OPO was always referenced relative to the $\Delta \theta=0.0^{\circ}$ point on this graph.

above $1950 \mathrm{~nm}$ is due to a change in reflectivity of the cavity mirrors. The dip in the OPO output energy at $1700 \mathrm{~nm}$ is where the OPO cavity mirrors have equal transmission and the dip at $1775 \mathrm{~nm}$ is due to strong $\mathrm{CO}_{2}$ and $\mathrm{H}_{2} \mathrm{O}$ absorptions in the air. Note that the tuning range of the $\mathrm{LiNbO}_{3} \mathrm{OPO}$ does not span the calculated degenerate wavelength for YCOB of $2100 \mathrm{~nm}$. However, given the uncertainty to this calculated value $(\sim \pm 200 \mathrm{~nm})$, as was discussed in section 4.2.3, we decided to proceed with experiments using the available OPO tuning range to see if possibly the COB crystals' phasematched SHG wavelengths lie in the $1650-1950 \mathrm{~nm}$ range. 


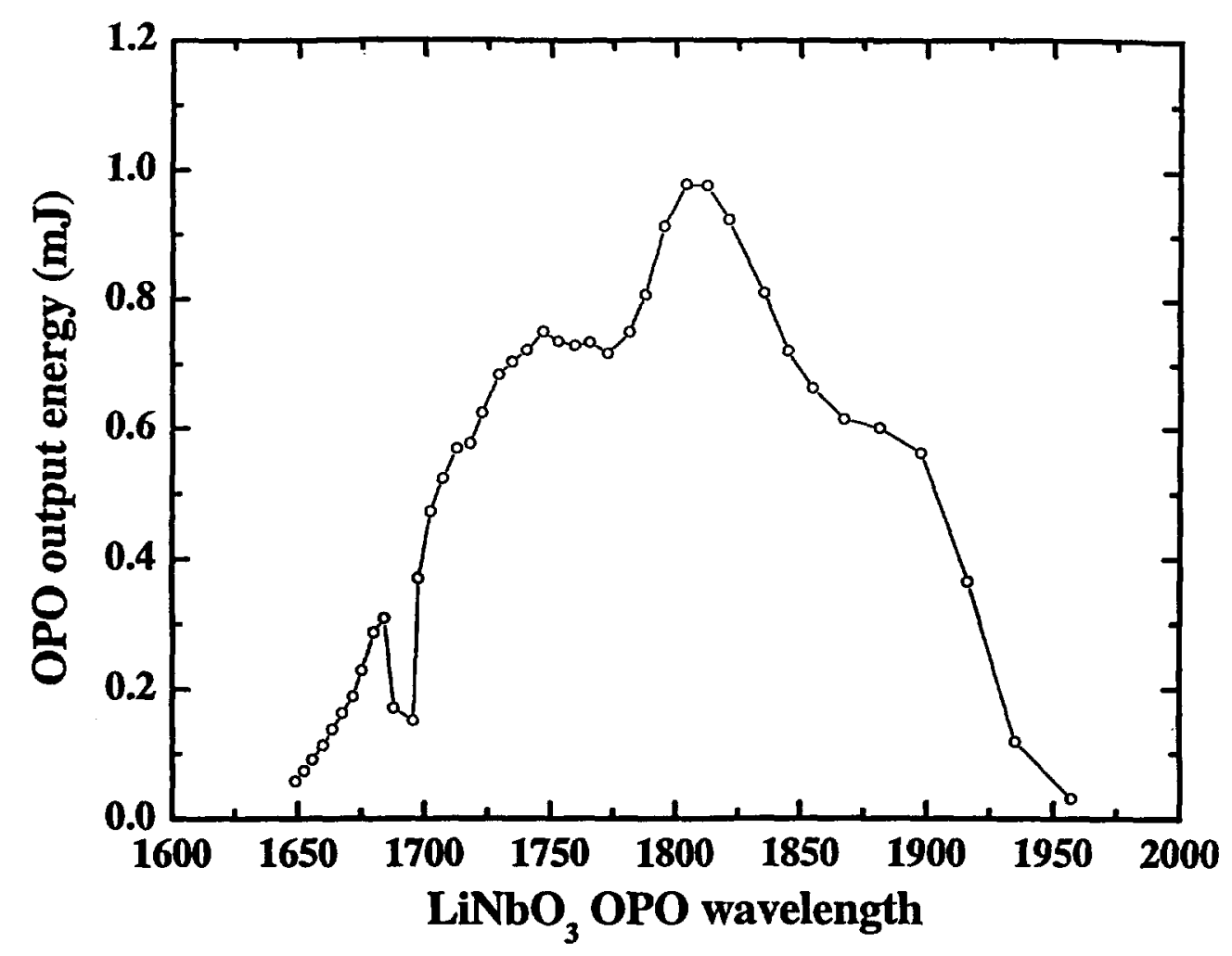

Figure 4.6. $\mathrm{LiNbO}_{3} \mathrm{OPO}$ output energy vs. signal wavelength. The line through the data points is only to aid the eye.

\subsubsection{SHG experiments with the $C O B$ crystals}

The second part of the experimental work in this chapter was to measure the type II non-critically phasematched SHG wavelengths for propagation along the $\mathrm{x}$ dielectric direction in the COB crystals. A schematic of the basic experiment was shown in Fig. 4.2. Fig. 4.7 shows the detailed experimental arrangement that we used.

The image relay telescope formed by the two plano-convex lenses has a demagnification of 2 and served two purposes. First, it roughly collimated the OPO signal beam that was incident on the COB crystals and secondly, it reduced the beam diameter by a factor of two thereby increasing the irradiance incident on the COB crystals by a factor of four. This increase in the irradiance of the OPO signal beam probing the 


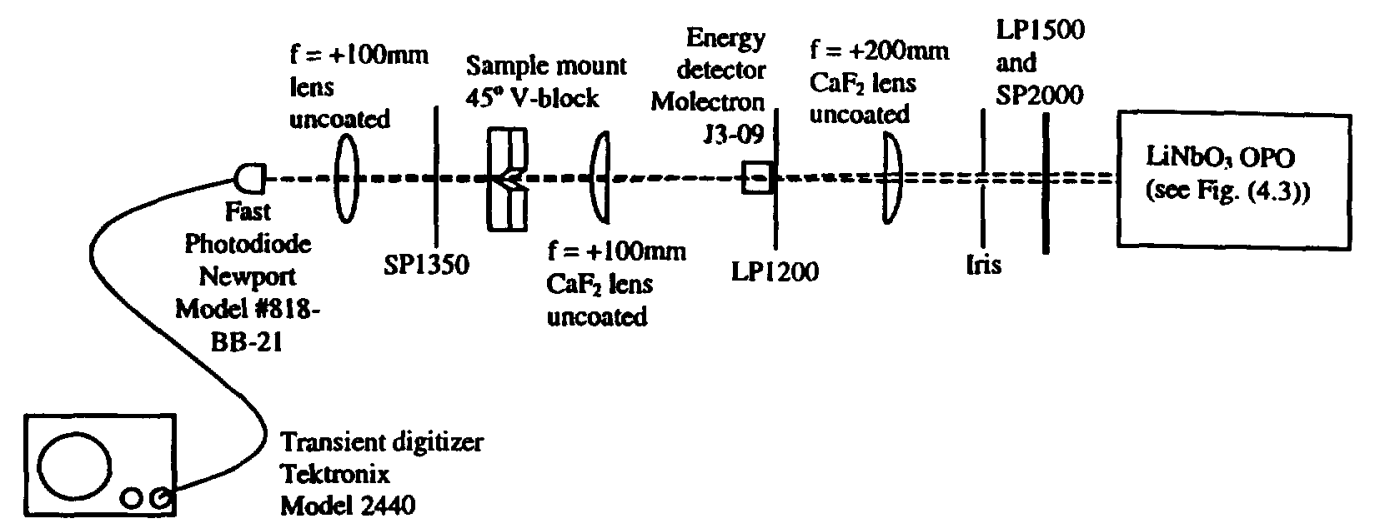

Figure 4.7. Experimental setup to measure the non-critical type II SHG wavelengths for propagation along the $x$ dielectric axis in the COB crystals. Note that the J3-09 energy meter and LP1200 filter were only placed in the beam path temporarily to measure the OPO output energy at each wavelength setting. The V-block was used to orient the COB crystals for type II interactions.

COB crystals helped boost the strength of the signal produced by the COB crystals to an easily detectable level ( $-200 \mathrm{mV}$ peak on the photodiode).

Two samples of YCOB, one sample of GdCOB, and one sample of LaCOB, were prepared with faces polished perpendicular to within 1 degree of the $\mathrm{x}$ dielectric axis. The faces of the two YCOB samples were polished to within 5 arc seconds of parallel. The faces of the GdCOB and the LaCOB samples were wedged by about 0.5 arc minute. The faces of all the crystals perpendicular to the $\mathrm{x}$ dielectric axis were prepared with a high quality optical polish. The GdCOB sample was cut from a commercially grown slab originally prepared for SHG at $1064 \mathrm{~nm}$. The direction of the $\mathrm{x}$ axis was determined in the slab using a polariscope and then a sample was cut in the form of a cube. The propagation lengths along the $\mathrm{x}$ dielectric axis for all four $\mathrm{COB}$ crystals are given in Table 4.1.

A $48.34 \mathrm{~mm} \mathrm{LiNbO}_{3}$ crystal was used to benchmark the OPO pumped SHG experiment. It was a commercially grown crystal originally cut for use in a $1.5-3.5 \mu \mathrm{m}$ 
Table 4.1. Crystals and propagation lengths used in the SHG non-critical wavelength experiments. *The propagation direction in the $\mathrm{LiNbO}_{3}$ crystal was at approximately $47^{\circ}$ from the optic axis in the yz principal plane.

\begin{tabular}{|c|c|}
\hline Crystal & $\begin{array}{c}\text { x-axis propagation } \\
\text { length (mm) }\end{array}$ \\
\hline YCOB & 9.54 \\
\hline YCOB & 17.81 \\
\hline GdCOB & 4.42 \\
\hline LaCOB & 3.80 \\
\hline LiNbO $_{3}$ & $48.34^{*}$ \\
\hline
\end{tabular}

OPO. The crystal was uncoated and one face was wedged relative to the other by about 7 arc minutes. The $\mathrm{LiNbO}_{3}$ crystal was used in two ways. First, its peak SHG response was measured for a given type I orientation. This gave a relative standard from which to predict the level of response from the COB crystals. Secondly, the FWHM of its SHG response as a function wavelength was measured (i.e. spectral sensitivity) to determine if the experiment was resolving the proper behavior due to the wavelength tuning of the pump wave.

Initially, since the $\mathrm{LiNbO}_{3} \mathrm{OPO}$ would only tune from 1650 to $1950 \mathrm{~nm}$, a preliminary experiment was conducted on the x-cut YCOB crystal to see if the SHG phasematched wavelength actually fell in this range. If no SHG signal could be detected from the YCOB crystal in this wavelength range, then the $\mathrm{LiNbO}_{3} \mathrm{OPO}$ optics would need to be changed to produce an output signal spanning $2100 \mathrm{~nm}$. The YCOB crystal was placed in the V-block in Fig. 4.7 and a Cohu CCD camera Model ER5001B was placed in the position of the photodiode. The camera was connected directly to a video monitor. The YCOB crystal was aligned approximately perpendicular to the OPO signal 
beam and then the OPO was tuned from 1950 to $1650 \mathrm{~nm}$. At around $1750 \mathrm{~nm}$, a noisy SHG signal was observed from the YCOB crystal on the video monitor. The signal was verified to be due to the $\mathrm{YCOB}$ crystal by removing and replacing the $\mathrm{YCOB}$ crystal and observing the signal to disappear and reappear. This initial observation of SHG around $1750 \mathrm{~nm}$ from the YCOB crystal was motivation for a more careful and methodical set of experiments to be conducted on the COB crystals in the wavelength range of $1650 \mathrm{~nm}$ to $1950 \mathrm{~nm}$. At this point in the study, the calculated value of the phasematched SHG pump wavelength of $2100 \mathrm{~nm}$ for YCOB was considered to be inaccurate and no further attempts were made to make measurements that spanned $2100 \mathrm{~nm}$.

The experimental procedure used to measure the SHG wavelengths in the 1650 to $1950 \mathrm{~nm}$ range for propagation along the $\mathrm{x}$ dielectric axis in the COB crystals will now be described. Each of the $\mathrm{COB}$ crystals and the $\mathrm{LiNbO}_{3}$ crystal was placed in the experiment in turn at the location of the V-block in Fig. 4.7. The COB crystals were placed in the V-block (type II SHG) and aligned in angle such that their polished faces were approximately perpendicular to the $\mathrm{OPO}$ pump beam. For the $\mathrm{LiNbO}_{3}$ crystal, the V-block was removed (type I SHG) and the crystal was tuned in angle to produce peak SHG for a pump wavelength of $1750 \mathrm{~nm}$.

After each crystal was aligned on the OPO pump beam, the OPO was tuned from 1950 to $1650 \mathrm{~nm}$ in steps of $0.05^{\circ}$ external angle $(\approx 7 \mathrm{~nm}$ per step). At each angle setting for the OPO, the SHG signal for the crystal under test was measured using the photodiode and transient digitizer (oscilloscope) shown in Fig. 4.7. The signal was averaged on the transient digitizer over 64 samples to help reduce the shot to shot noise in the reading. After the reading was made from the transient digitizer, the J3-09 energy meter was 
placed in position, as shown in Fig. 4.7, and that signal was averaged over 40 shots to give the OPO incident energy. Then the OPO was tuned to a shorter wavelength by $0.05^{\circ}$ and the procedure was repeated.

The raw SHG data collected for each of the crystals is shown in Fig. 4.8. The raw data was normalized to variances in the OPO pump irradiance by dividing it by the square of the normalized OPO pump energy. The data was then further normalized by the SP1350 filter response in the range of the SHG produced by each crystal under test. The normalized data is shown in Fig. 4.9. The lines connecting the points in Figs. 4.8 and 4.9 are only to serve as an aid to the eye. All of the COB crystals show a peak SHG response at $-1725 \mathrm{~nm}$ in the raw data. In the normalized data, a peak SHG response is still observable but much less obvious.

\subsection{Discussion of the SHG response}

The appearance of the data for the $C O B$ crystals in Figs. 4.8 and 4.9 is very unexpected since it does not have a sinc ${ }^{2}$ response with wavelength. We estimated the SHG conversion efficiency to be approximately $1.5 \%$, which would imply that the response with wavelength should be a sinc ${ }^{2}$ as described by Eqn. (2.52).) Also, the SHG response for the COB crystals in Figs. 4.8 and 4.9 shows an unusual "spiking" behavior as the pump wavelength was tuned. The SHG measurements on all the crystals were repeated several times with the results (i.e. the SHG signal strength and the spike

positions) for each crystal being almost identical between the data sets (i.e. very reproducible). Therefore the cause of the fluctuations in the COB crystal's data was not a random or time dependent effect.

This prompted efforts to determine if what was observed was actually phasematched 


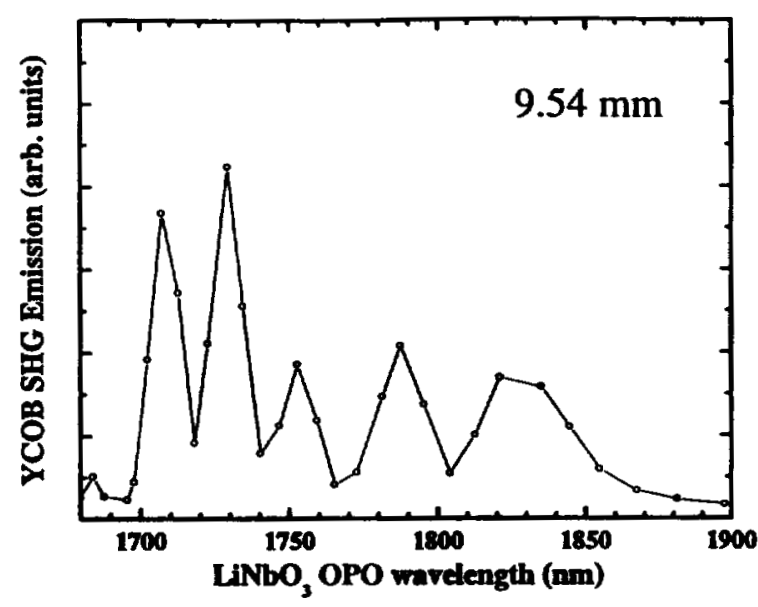

(a)

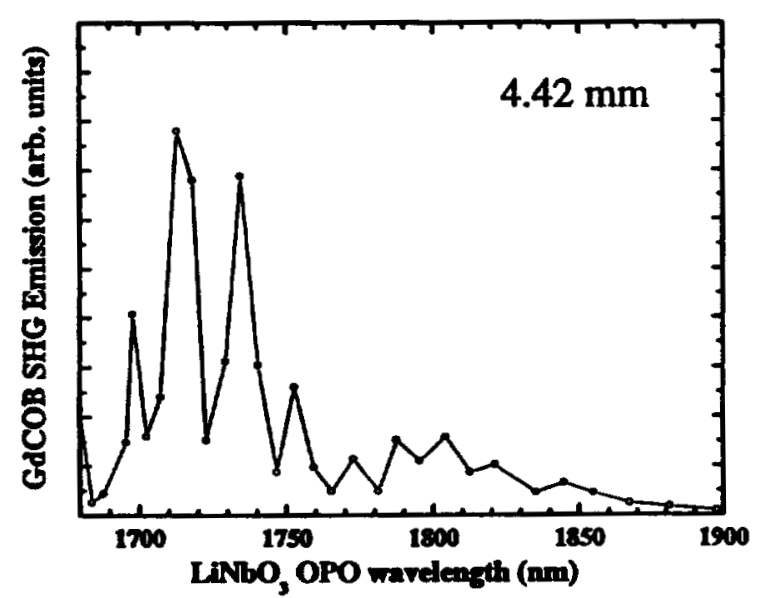

(c)

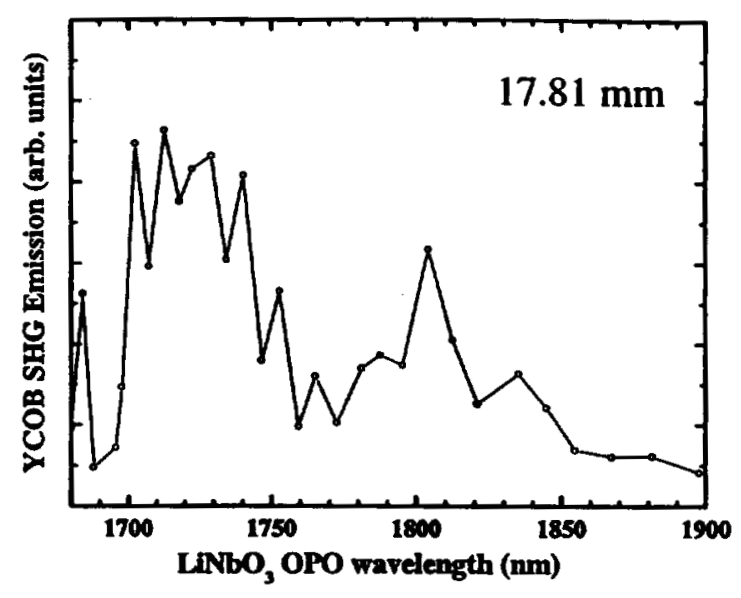

(b)

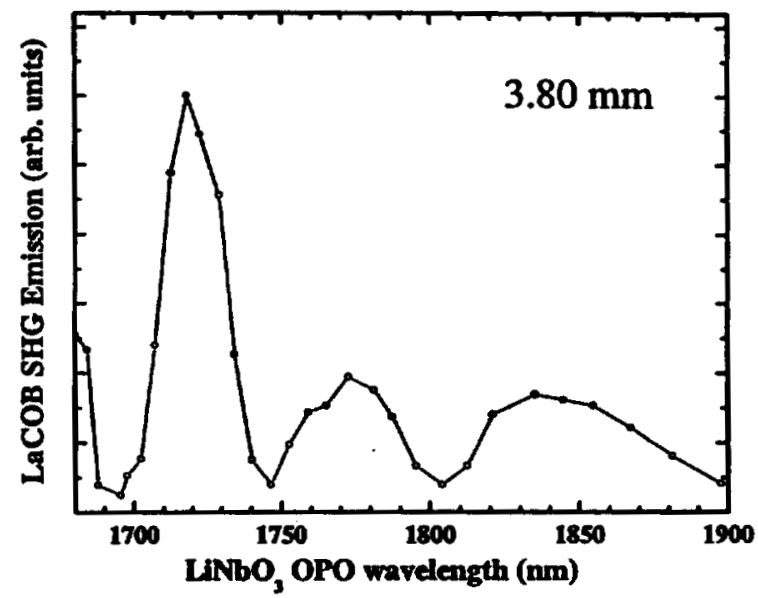

(d)

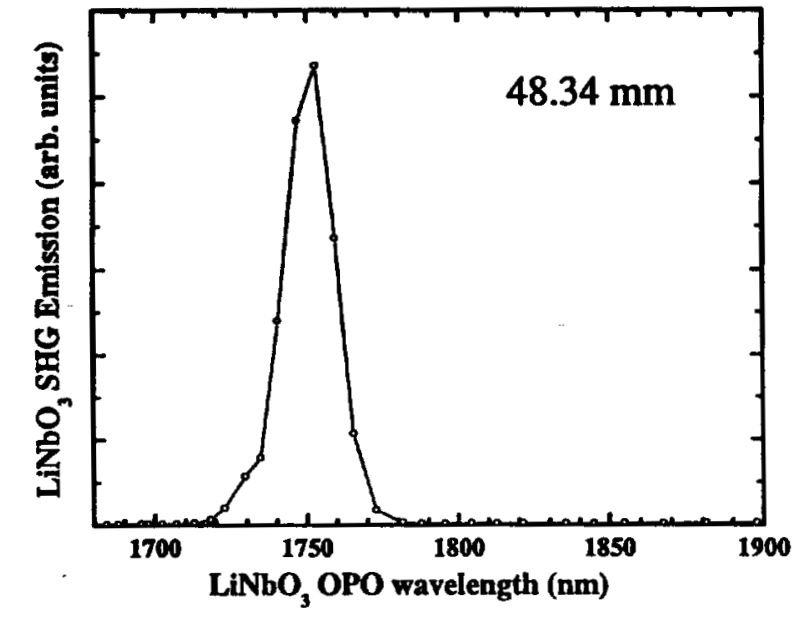

(e)

Figure 4.8. Measured raw data for the SHG responses. (a) $9.54 \mathrm{~mm}$ YCOB crystal, (b) $17.81 \mathrm{~mm}$ YCOB crystal, (c) $4.42 \mathrm{~mm}$ GdCOB crystal, (d) $3.80 \mathrm{~mm}$ LaCOB crystal, and (e) $48.34 \mathrm{~mm} \mathrm{LiNbO}$ crystal. 

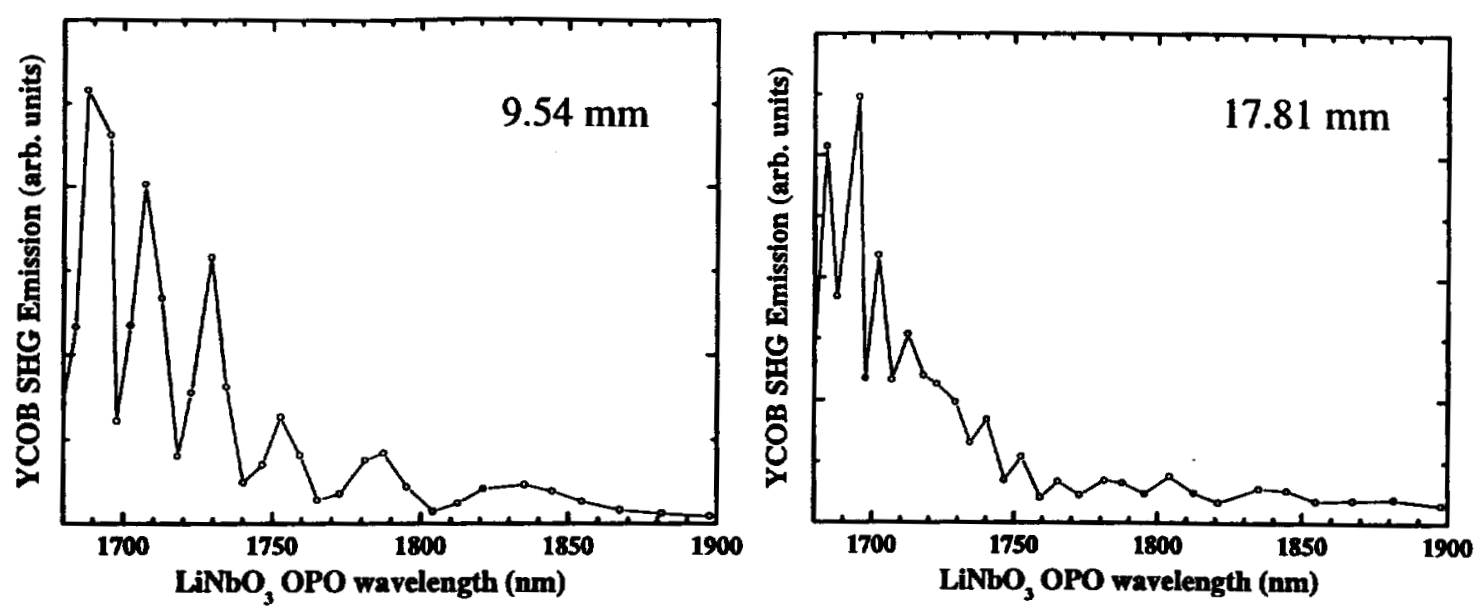

(a)

(b)

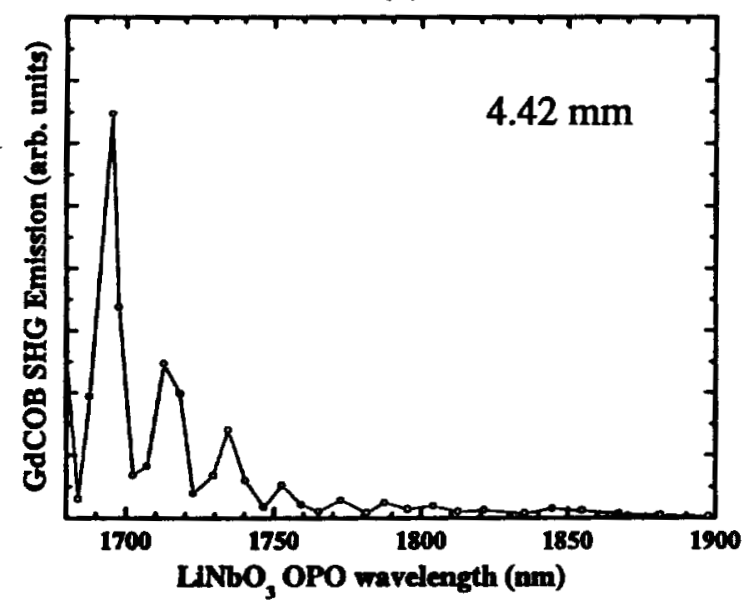

(c)

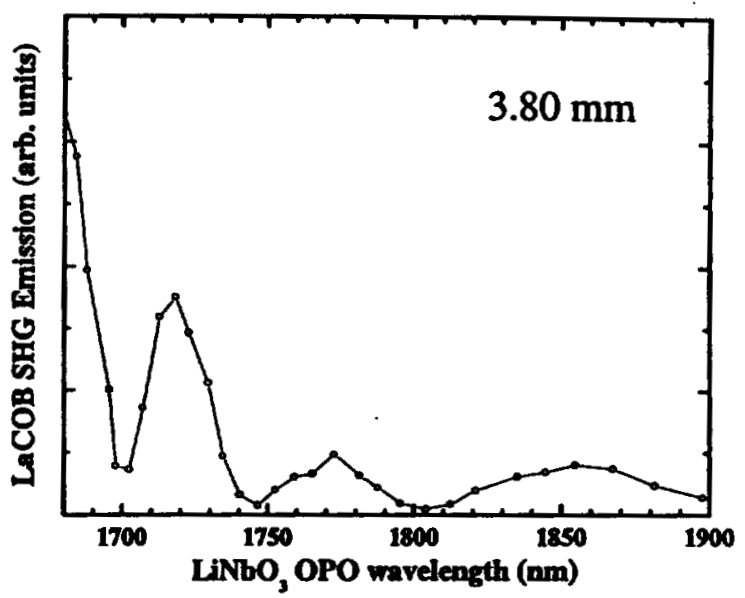

(d)

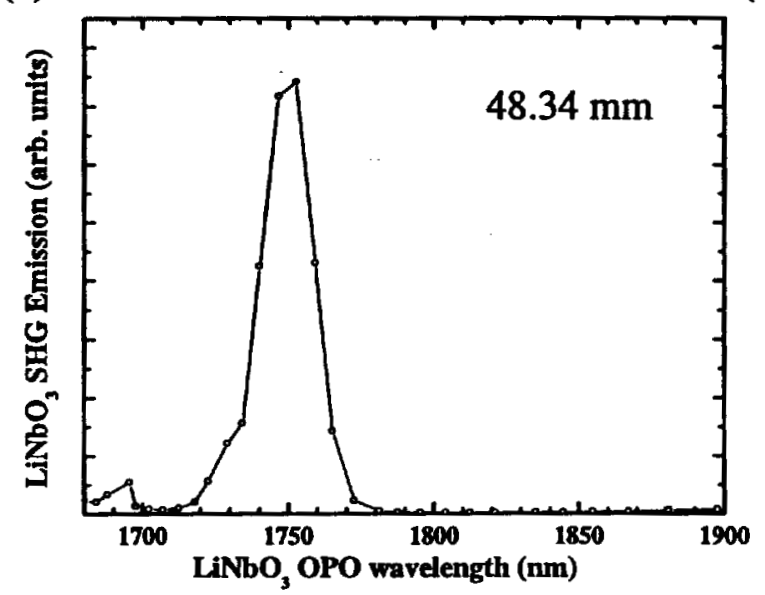

(e)

Figure 4.9. Normalized SHG responses. (a) $9.54 \mathrm{~mm}$ YCOB crystal, (b) $17.81 \mathrm{~mm}$ YCOB crystal, (c) $4.42 \mathrm{~mm}$ GdCOB crystal, (d) $3.80 \mathrm{~mm}$ LaCOB crystal, and (e) 48.34 mm $\mathrm{LiNbO}_{3}$ crystal. 
SHG. Several experimental "checks" were performed to determine if the signals from the COB crystals were phasematched SHG. First, with the OPO tuned such that the x-cut YCOB crystal was producing a signal, the $x$-cut YCOB crystal was replaced by a z-cut YCOB crystal (no type II SHG theoretically allowed along the $\mathrm{z}$ direction) of about the same thickness and the observed SHG signal vanished completely. Second, the $\mathrm{x}$-cut YCOB crystal in the type II orientation was rotated 45 degrees and the SHG signal was observed to drop to zero indicating that a true type II SHG signal was being observed. Next, with the YCOB crystal producing a signal, an approximately 0.3 O.D. filter was placed into the OPO pump beam ahead of the YCOB crystal and the SHG signal was observed to drop by about a factor of 4 . These tests lead to the conclusion that the observed signals were phasematched SHG generated by the COB crystals.

In order to add further credibility to the SHG data for the COB crystals, the ratio of the normalized measured peak SHG signal for one of the YCOB crystals, (a) in Fig. 4.8, was formed with that of the $\mathrm{LiNbO}_{3}$ crystal and that ratio was then compared to the theoretically calculated ratio of $d_{\text {eff }}{ }^{2} L^{2} / n(2 \omega) n^{2}(\omega)$ for the two materials. The ratio of the normalized measured peak SHG signals from the two crystals is calculated in Eqn. (4.11) from the experimental data. The peak SHG signals for both the $\mathrm{LiNbO}_{3}$ and $\mathrm{YCOB}$

$$
\frac{\mathrm{I}_{2 \omega}^{\mathrm{YCOB}}}{\mathrm{I}_{2 \omega}^{\mathrm{LNbO}}}=0.0079 \pm 20 \%
$$

crystals were estimated from the data and each had 5\% and 15\% experimental uncertainty, respectively. The peak SHG signal for the $\mathrm{LiNbO}_{3}$ crystal was fairly easy to identify from the data collected, however, the peak for the YCOB crystal was difficult to determine. The value used in the calculation of Eqn. (4.11) was determined by 
examining the raw YCOB data and estimating, from the wavelength span of highest SHG response, what the average peak SHG signal level was and its associated uncertainty. The peak value was then normalized and used in Eqn. (4.11). The theoretical ratio of the corresponding $\mathrm{d}_{\mathrm{eff}}{ }^{2} \mathrm{~L}^{2} / \mathrm{n}(2 \omega) \mathrm{n}^{2}(\omega)$ for the two materials is calculated in Eqn. (4.12). The

$$
\begin{aligned}
& \frac{\mathrm{d}_{\mathrm{eff}}^{2}(\mathrm{YCOB}) \mathrm{L}^{2}(\mathrm{YCOB}) \mathrm{n}_{2 \omega}\left(\mathrm{LiNbO}_{3}\right) \mathrm{n}_{1 \omega}^{2}\left(\mathrm{LiNbO}_{3}\right)}{\mathrm{d}_{\mathrm{eff}}^{2}\left(\mathrm{LiNbO}_{3}\right) \mathrm{L}^{2}\left(\mathrm{LiNbO}_{3}\right) \mathrm{n}_{2 \omega}(\mathrm{YCOB}) \mathrm{n}_{1 \omega}^{2}(\mathrm{YCOB})}= \\
& \frac{(1.69 \mathrm{pm} / \mathrm{V})^{2}(9.54 \mathrm{~mm})^{2}(2.17)(2.20)^{2}}{(5.12 \mathrm{pm} / \mathrm{V})^{2}(48.3 \mathrm{~mm})^{2}(1.7)^{3}}=0.0092
\end{aligned}
$$

value for the ratio in Eqn. (4.12) agrees within the experimental uncertainty with the value in Eqn. (4.11). Therefore it is again concluded that the signal from the YCOB crystal is phasematched SHG. Given the general level of the measured signal from the other COB crystals, it can be concluded that those crystals too, produced phasematched SHG.

The results for the response of the $\mathrm{LiNbO}_{3}$ crystal in Figs. 4.8 and 4.9 do resemble a $\operatorname{sinc}^{2}$. A fit of Eqn. (2.52) to the data in Fig. 4.9 (e) yields a wavelength sensitivity $\left(\beta_{\lambda}\right)$ of $0.054(\mathrm{~cm}-\mathrm{nm})^{-1}$ for $\mathrm{LiNbO}_{3}$. This value is about 125 times less than the calculated value of $6.63(\mathrm{~cm}-\mathrm{nm})^{-1}$. Therefore the response of the $\mathrm{LiNbO}_{3}$ crystal is 125 times wider in wavelength than expected. We would expect a very clear $\operatorname{sinc}^{2}$ response that has the width we expect for the $\mathrm{LiNbO}_{3}$ crystal because $\mathrm{LiNbO}_{3}$ is a perfected material and our crystal was commercially produced. Since the width of the response even for $\mathrm{LiNbO}_{3}$ is much too wide, this implies that there must be some errors in our assumptions about our experimental procedure.

We are assuming that our OPO pump beam is monochromatic. This is highly 
unlikely for two reasons. First, the OPO cavity is composed of flat mirrors and so there is no distinct cavity mode that is supported-i.e. the OPO probably oscillates in many modes. Secondly, the $\mathrm{Nd}$ :YAG beam is not absolutely collimated at the $\mathrm{LiNbO}_{3}$ crystal. Therefore the presence of many cavity modes combined with a non-collimated pump beam would allow non-collinear phasematching to occur in the $\mathrm{LiNbO}_{3}$ OPO producing an output beam that was broadband and diverging [9]. Therefore these two issues (broadband and diverging) with the $\mathrm{LiNbO}_{3}$ beam, would give rise to non-collinear phasematching in our test crystals which is an effect that could be responsible for the spectrally broad SHG signals obtained for the $\mathrm{LiNbO}_{3}$ and $\mathrm{COB}$ crystals.

The spiking behavior in the $C O B$ crystal's data could possibly be due to an interference or etalon effect arising in the crystals. To obtain an estimate for the level at which an etalon effect might affect the transmission of the crystals, the free spectral range, finesse, and cavity mode width were calculated for an etalon with properties (thickness, index of refraction, and reflectivity) that are representative of the $\mathrm{COB}$ crystals studied. Following [10] and [11], for an etalon with a thickness of $0.954 \mathrm{~cm}$, an index of refraction of 1.7 , and a reflectivity of 0.067 (Fresnel reflection) the free spectral range, finesse, and cavity mode width can be calculated to be $0.09 \mathrm{~nm}$ (at $1750 \mathrm{~nm}$ ), 0.87 , and $10 \mathrm{GHz}$, respectively. A free spectral range of $0.09 \mathrm{~nm}$ corresponds to a spacing between adjacent transmission peaks that is much too narrow to be resolved by the SHG experiments since the $\mathrm{LiNbO}_{3}$ OPO output had a linewidth of $1-6 \mathrm{~nm}$. The modulation depth of the transmitted irradiance as a function of wavelength through an etalon with a Finesse of 0.87 should be a maximum of $50 \%$. As can be seen from the data in Figs. 4.8 and 4.9 , the SHG signal dips well below the $50 \%$ level in most cases. It therefore seems 
unlikely that the effect causing the fluctuations in the COB crystals' data is an etalon effect.

From the data presented and the preceding discussion, it is clear that the measured SHG data for the COB crystals does not follow the expected behavior. Further experimental work is needed to accurately determine the type II NCPM wavelengths for propagation along the $\mathrm{x}$ dielectric axis in $\mathrm{YCOB}, \mathrm{GdCOB}$, and $\mathrm{LaCOB}$ in order to evaluate their utility for OPO applications. Steps should be taken to narrow the linewidth of the OPO output and to achieve better beam collimation at the samples. The linewidth of the OPO could be improved by using cavity mirrors that make the OPO cavity into a stable configuration. The OPO pump beam also needs to be examined to see if it is maintaining a constant spatial profile as the OPO is tuned. The tuning range of the OPO needs to be extended to shorter wavelengths to investigate if the COB crystals' response might actually peak at a wavelength shorter than $1650 \mathrm{~nm}$. Also the tuning range of the OPO needs to be extended to longer wavelengths to verify that the phasematched SHG wavelength is not actually near $2100 \mathrm{~nm}$ as predicted by the Sellmeier equations of Ref. [3]. Wedging of the faces of the crystals should be explored to determine if this would reduce the fluctuations seen here in the SHG signals.

\subsection{Summary}

We successfully built a pulsed $\mathrm{LiNbO}_{3}$ OPO that produced 4 ns pulses with a maximum energy per pulse of $1.0 \mathrm{~mJ}$ at $1825 \mathrm{~nm}$. The beam quality of the Nd:YAG laser used to pump the $\mathrm{LiNbO}_{3}$ OPO was experimentally characterized using an $\mathrm{M}^{2}$ analysis, and was found to have $\mathrm{M}^{2}$ values of 2.206 and 2.081 in the horizontal and 
vertical directions, respectively. We were also able to continuously angle tune the $\mathrm{LiNbO}_{3} \mathrm{OPO}$ from 1625 to $1975 \mathrm{~nm}$.

Using the $\mathrm{LiNbO}_{3} \mathrm{OPO}$ as a pump source, type II phasematched SHG was observed for propagation along the $x$ dielectric axis in $\mathrm{YCOB}, \mathrm{GdCOB}$, and $\mathrm{LaCOB}$. Attempts to determine the exact non-critically phasematched wavelength down the $\mathrm{x}$ dielectric axis for these three crystals were not successful. The data collected for the COB crystals showed a phasematched signal but the phasematched wavelengths could only be estimated to be near $1725 \mathrm{~nm}$ and the expected sinc $^{2}$ response could not be resolved.

$\mathrm{A} \mathrm{LiNbO}_{3}$ crystal produced data that had an approximate $\operatorname{sinc}^{2}$ form and allowed determination of the wavelength sensitivity through a numerical fit to the data. A value for $\beta_{\lambda}$ of $0.054(\mathrm{~cm}-\mathrm{nm})^{-1}$ was determined for a $4.83 \mathrm{~cm}$ crystal. This experimentally determined value for $\beta_{\lambda}$ for $\mathrm{LiNbO}_{3}$ differs greatly from the calculated value of 6.63 (cm$\mathrm{nm})^{-1}$. Lack of monochromaticity and collimation of the OPO pump beam were discussed as possible causes to the broad response for the $\mathrm{LiNbO}_{3}$ and the $\mathrm{COB}$ crystals. To more accurately determine the SHG wavelengths for the COB crystals, several improvements to the experimental procedure were suggested. 


\section{References for Chapter 4}

[1] S. P. Velsko, M. Ruggiero, and M. Hermann, in Application of Lidar to Current Atmospheric Topics, A. J. Sedlacek, ed., Proc. SPIE 2833, 144 (1996)

[2] M. S. Webb, K. B. Stanion, D. J. Deane, W. A. Cook, W. A. Neuman, and S. P. Velsko, in Nonlinear Frequency Generation and Conversion, M. C. Gupta, W. J. Kozlovsky, D. C. MacPherson, eds., Proc. SPIE 2700, 269 (1996)

[3] M. Iwai, T. Kobayashi, H. Furuya, Y. Mori, and T. Sasaki, Jpn. J. Appl. Phys. 36, L276 (1997)

[4] F. Mougel, et al., Optical Society of America Trends in Optics and Photonics Series 26, 709 (1999)

[5] S. J. Brosnan and R. L. Byer, IEEE J. Quan. Elec., 15, 415 (1979)

[6] M. Runkel, private communication, LLNL (2001)

[7] R. Beach, private communication, LLNL (2001)

[8] R. L. Herbst, R. N. Fleming, and R. L. Byer, Appl. Phys. Lett. 25, 520 (1974)

[9] F. Zernike and J. E. Midwinter, Applied Nonlinear Optics, (John Wiley and Sons, Inc., New York, 1973)

[10] E. Hecht, Optics, $3^{\text {rd }}$ ed. (Addison-Wesley, Reading, Massachusetts, 1998), Chapter 9

[11] A. E. Siegman, Lasers, (University Science Books, Sausalito, California, 1986), Chapter 11 


\section{Chapter 5}

\section{Linear electro-optic properties of $\mathrm{YCa}_{4} \mathrm{O}\left(\mathrm{BO}_{3}\right)_{3}(\mathrm{YCOB})$}

Originally titled as: J. J. Adams and C. A. Ebbers, "Linear electro-optic properties of $\mathrm{YCa}{ }_{4} \mathrm{O}\left(\mathrm{BO}_{3}\right)_{3}$," to be published

\subsection{Introduction}

In the past five years, several new nonlinear crystals (i.e. $\mathrm{YCa}_{4} \mathrm{O}\left(\mathrm{BO}_{3}\right)_{3}[1-4]$, $\mathrm{CsLiB}_{6} \mathrm{O}_{10}[4]$, and $\left.\mathrm{BiB}_{3} \mathrm{O}_{6}[5]\right)$ have been discovered and commercially developed for use as frequency doublers and triplers. However, there remains little progress in expanding the suite of commercially available electro-optic crystals. Crystals for use in commercial electro-optic applications must have the standard properties that make them desirable as optical materials (i.e. lack of hygroscopicity, high damage threshold, high transparency within the desired wavelength range, favorable growth and fabrication properties, etc.) and they must also possess adequate nonlinear optical coefficients. In the absence of an ionic contribution to the nonlinear susceptibility $\left(\chi^{(2)}\right)[6]$, the electro-optic coefficient of a material $\left(\mathrm{r}_{\mathrm{ii}}\right)$ is strictly proportional to the value of the nonlinear optical coefficient $\left(d_{i l}\right)[7-9]$ as given by

$$
r_{\mathrm{li}} \cong \frac{4 d_{i 1}(\varepsilon-1)}{\left(n^{2}-1\right) n^{4}}
$$

where $\varepsilon$ is the linear dielectric constant and $\mathrm{n}$ is the refractive index of the material. Thus without a ferroelectric phase transition, only nonlinear crystals with substantial nonlinear optical coefficients $\left(d_{i l}\right)$ have merit as electro-optic crystals.

The half-wave voltage $\left(V_{\pi}\right)$ for an electro-optic crystal (as was discussed in Chapter 2) is given by Eqn. (2.134) as 


$$
\mathrm{V}_{\pi}=\frac{\mathrm{d} \lambda}{\mathrm{L} \mathrm{r}_{\mathrm{eff}}}
$$

where $d$ is the separation between the electrodes (i.e. the crystal thickness over which the DC voltage is applied), $\mathrm{L}$ is the beam propagation length through the crystal, $\lambda$ is the wavelength of the light, and $r_{\text {eff }}$ is the effective electro-optic coefficient. The form of the effective electro-optic coefficient is dependent upon the crystal symmetry, as well as the orientation of the applied voltage and direction of light propagation. In general, it is desirable to use applied voltages less than $15 \mathrm{kV}$, otherwise corona discharge begins to arise as an issue. In addition, material aspect ratios larger than 10:1 (L:d) become impractical from a propagation and device fabrication point of view. With these two constraints in mind, potentially useful electro-optic crystals should have effective electrooptic coefficients of at least $7 \mathrm{pm} / \mathrm{V}$.

KTiOPO 4 [10], $\mathrm{KD}_{2} \mathrm{PO}_{4}(\mathrm{KD} * \mathrm{P})$ [11], $\mathrm{LiNbO}_{3}$ [12], and $\mathrm{BaB}_{2} \mathrm{O}_{4}(\mathrm{BBO})$ [13] are four common commercially available electro-optic crystals which in a typical configuration have effective electro-optic coefficients of approximately $170,160,135$, and $20 \mathrm{pm} / \mathrm{V}$, respectively. Of these 4 crystals, only $K D^{*} \mathrm{P}$ is routinely available with high optical homogeneity for apertures exceeding $2 \mathrm{~cm}^{2}$. Unfortunately, due to relatively large stressoptic coefficients [6], low thermal conductivity [14], and difficulty in accepting optical coatings, KD*P is not well suited for high-average power applications. Thus it is of interest to characterize the electro-optic coefficients of new nonlinear optical crystals with favorable growth, fabrication, and high-average power handling properties.

In Chapter 3, we determined that the values for the $d_{12}$ and $d_{32}$ nonlinear optical coefficients for $\mathrm{LaCa}_{4} \mathrm{O}\left(\mathrm{BO}_{3}\right)_{3}, \mathrm{GdCa} a_{4} \mathrm{O}\left(\mathrm{BO}_{3}\right)_{3}$, and $\mathrm{YCOB}$ vary very little between the 
three crystals. The $d_{32}$ coefficient was found to be of relatively large magnitude equal to $1.69 \mathrm{pm} / \mathrm{V}$. Eqn. (5.1) can be used to estimate the magnitude of the corresponding linear electro-optic coefficient $\left(r_{23}\right)$ for YCOB. We have estimated the dielectric constant $(K)$ for YCOB to be $\approx 8$ from a simple parallel-plate capacitance measurement. Therefore using Eqn. (5.1) with $K=8$ and $n=1.7$, we estimate that the corresponding linear electro-optic coefficient $\left(\mathrm{r}_{23}\right)$ would have a magnitude of approximately $3 \mathrm{pm} / \mathrm{V}$. This implies that the corresponding effective electro-optic coefficient $\left(\mathrm{r}_{\mathrm{eff}}\right)$ would have an approximate magnitude of $\mathrm{r}_{23} \mathrm{n}^{3} \approx 14 \mathrm{pm} / \mathrm{V}$. Because of their robust mechanical and thermo-mechanical nature [15], these materials-if they do indeed possess substantial linear electro-optic coefficients-would have potential application in large aperture, highaverage power $1 \mu \mathrm{m}$ Q-switches.

In this chapter, we report on measurements of the effective linear electro-optic coefficients along principal dielectric directions in YCOB. Section 5.2 describes the experimental techniques that were used to measure the coefficients. Section 5.3 presents the experimental results for several of the transverse and longitudinal YCOB modulators that were studied. In section 5.4, we discuss the experimental results and calculate the effective electro-optic coefficients and half-wave voltages. Section 5.5 summarizes the chapter.

\subsection{Experimental technique}

We performed two basic sets of experiments. One set of experiments involved measuring the transmitted irradiance $\left(\mathrm{I}_{\mathrm{trx}}\right)$ through a $\mathrm{KDP}, \mathrm{KD} * \mathrm{P}$, and six different YCOB modulators while a low frequency $\underline{\mathrm{AC}}$ voltage was applied. The other set of 
experiments involved measuring the transmitted irradiance $\left(\mathrm{I}_{\mathrm{trx}}\right)$ through a $\mathrm{KDP}, \mathrm{KD} * \mathrm{P}$, and six different $\mathrm{YCOB}$ modulators while a $\underline{\mathrm{DC}}$ voltage was applied.

The YCOB samples used in the experiments were cut from a Czochralski grown boule that was procured from Crystal Photonics, Inc. Fl. The YCOB samples were fabricated into slabs with polished faces perpendicular to the crystal's dielectric directions $(x, y$, and $z$ ) as specified in Tables 5.1 and 5.2. The crystal faces across which the electric field was applied were sputter-coated with approximately $2 \mu \mathrm{m}$ of gold for the transverse cases and $120 \mathrm{~nm}$ of gold for the longitudinal cases. (The thinner gold coating that was used for the longitudinal cases was electrically conductive and $\sim 50 \%$ transmissive.)

Electro-optic experiments were also conducted on a $\mathrm{KH}_{2} \mathrm{PO}_{4}(\mathrm{KDP})$ crystal and a KD*P crystal. These crystals were used as standards for comparison. The KDP crystal was grown at LLNL and had uncoated polished faces perpendicular to the directions specified in Table 5.3. The $K D * P$ crystal was extracted from a commercially manufactured Q-switch and had uncoated polished faces perpendicular to the directions specified in Table 5.3. We did not have any literature on the deuteration level of the $\mathrm{KD} * \mathrm{P}$ crystal nor did we perform any analysis to determine it. However since the KD*P crystal was obtained from a commercially produced Pockels cell, we will assume it has a deuteration level of $99 \%$ as is often found in commercially produced $\mathrm{KD} * \mathrm{P}$ for electrooptic applications [16].

For the electro-optic experiments, transverse and longitudinal configurations were studied as schematically shown in Chapter 2, Figs. 2.12 and 2.14. In Chapter 2, we derived expressions that related the transmitted irradiance of six different YCOB modulators to the applied voltage. We quote the expressions derived in Chapter 2 
(Tables 2.1 and 2.2) for the YCOB modulators in Tables 5.1 and 5.2. The expressions for $\mathrm{I}_{\mathrm{trx}}$ that are shown in Table 5.3 for the $\mathrm{KDP}$ and $\mathrm{KD} * \mathrm{P}$ modulators can be derived using a very similar method to that described in Chapter 2 for the YCOB modulators. Several conventions are used in Tables 5.1 - 5.3 and throughout the rest of this chapter. First, the notation L:[\#\#] and $d:[\# \#]$ specify the directions for the beam propagation and the applied voltage, respectively in terms of the crystal's dielectric directions [xyz] (for the case of $\mathrm{KDP}$ and $\mathrm{KD}^{*} \mathrm{P}, \mathrm{x}$ and $\mathrm{y}$ are the two mutually orthogonal twofold axes of symmetry that lie in the plane normal to the optic axis $(z))$. $I_{0}$ is the irradiance of the laser beam entering the modulator. $\Gamma_{c}$ is the retardation imparted by the compensator which is manually adjustable from 0 to $6 \pi . \Gamma_{s}$ is the retardation due to the crystal's static birefringence and is defined in general by Eqn. (2.145) $\left(\Gamma_{\mathrm{xtal}} \equiv \Gamma_{\mathrm{s}}+\Gamma_{\mathrm{v}}\right)$.

In the expressions for $I_{t x x}$ for the YCOB modulators, $n_{x}, n_{y}$, and $n_{z}$ are the principal refractive indices for $Y C O B$ and have values at $632.8 \mathrm{~nm}$ of $n_{x}=1.695, n_{y}=1.727$, and $n_{z}=1.738$ and at $1064 \mathrm{~nm}$ they have values of $n_{x}=1.684, n_{y}=1.715$, and $n_{z}=1.725[1]$. In the expressions for $\mathrm{I}_{\mathrm{trx}}$ for the KDP and $\mathrm{KD} * \mathrm{P}$ modulators, $\mathrm{n}_{\mathrm{0}}$ is the ordinary refractive index and $n_{e}$ is the extraordinary refractive index. The values for $n_{0}$ and $n_{e}$ for KDP at $632.8 \mathrm{~nm}$ are 1.507 and 1.466 , respectively and at $1064 \mathrm{~nm}$ they have values of 1.494 and 1.460 , respectively [12]. The values for $n_{0}$ and $n_{e}$ for $K D^{*} P(99 \%$ deuteration) at 632.8 $\mathrm{nm}$ are 1.503 and 1.465 , respectively and at $1064 \mathrm{~nm}$ they have values of 1.493 and 1.458 , respectively [16].

The experimental setup used for the $\mathrm{AC}$ measurements is shown in Fig. 5.1. The quarter-wave plate $(\lambda / 4)$ ensured that circularly polarized light was incident on the crystal 
Table 5.1. Modified refractive indices, crystal retardations, and $I_{\operatorname{txx}}$ for four transverse YCOB modulators.

\begin{tabular}{|c|c|c|c|}
\hline $\begin{array}{c}\text { Crystal } \\
\text { Orientation } \\
L:[x y z] \\
d:[x y z] \\
\end{array}$ & $\begin{array}{l}n_{p}^{\prime \prime} \\
n_{q}^{\prime \prime}\end{array}$ & $\Gamma_{\text {xtal }}=\frac{2 \pi L}{\lambda}\left(n_{p}^{\prime \prime}-n_{q}^{\prime}\right)$ & $\left(\mathrm{V}_{\text {applied }} \mathrm{I}_{\mathrm{tx}} \ll \mathrm{V}_{\pi}\right)$ \\
\hline $\begin{array}{l}\mathrm{L}:[100] \\
\mathrm{d}:[001]\end{array}$ & $\begin{array}{l}\mathrm{n}_{\mathrm{z}}^{\prime \prime}=\mathrm{n}_{\mathrm{z}} \cdot \frac{\mathrm{r}_{33} \mathrm{n}_{\mathrm{z}}^{3} \mathrm{E}}{2} \\
\mathrm{n}_{\mathrm{y}}^{\prime \prime}=\mathrm{n}_{\mathrm{y}} \cdot \frac{\mathrm{r}_{23} \mathrm{n}_{\mathrm{y}}^{3} \mathrm{E}}{2}\end{array}$ & $\frac{2 \pi \mathrm{L}}{\lambda}\left(n_{z}-n_{y}\right)+\frac{L}{d} \frac{\pi}{\lambda}\left(I_{23} n_{y}^{3}-r_{33} n_{z}^{3}\right) V_{\text {applied }}$ & $\frac{I_{o}}{2}\left(1+\sin \Gamma_{c} \cos \Gamma_{s}-\sin \Gamma_{c} \sin \Gamma_{s} \frac{L}{d} \frac{\pi}{\lambda}\left(r_{23} n_{y}^{3}-\Gamma_{33} n_{z}^{3}\right) V_{\text {applied }}\right.$ \\
\hline $\begin{array}{l}\mathrm{L}:[010] \\
\mathrm{d}:[100]\end{array}$ & $\begin{array}{l}\mathrm{n}_{\mathrm{z}}^{\prime \prime}=\mathrm{n}_{\mathrm{z}}-\frac{\mathrm{r}_{31} \mathrm{n}_{\mathrm{z}}^{3} \mathrm{E}}{2} \\
\mathrm{n}_{\mathrm{x}}^{\prime \prime}=\mathrm{n}_{\mathrm{x}}-\frac{\mathrm{r}_{11} \mathrm{n}_{\mathrm{x}}^{3} \mathrm{E}}{2}\end{array}$ & $\frac{2 \pi L}{\lambda}\left(n_{z}-n_{x}\right)+\frac{L}{d} \frac{\pi}{\lambda}\left(r_{11} n_{x}^{3}-r_{31} n_{2}^{3}\right) V_{\text {applied }}$ & $\frac{I_{0}}{2}\left(1+\sin \Gamma_{c} \cos \Gamma_{s}-\sin \Gamma_{c} \sin \Gamma_{s} \frac{L}{d} \frac{\pi}{\lambda}\left(\Gamma_{11} n_{x}^{3}-\Gamma_{31} n_{z}^{3}\right) V_{\text {applied }}\right.$ \\
\hline $\begin{array}{l}\mathrm{L}:[010] \\
\mathrm{d}:[001]\end{array}$ & $\begin{array}{l}\mathrm{n}_{\mathrm{z}}^{\prime \prime}=\mathrm{n}_{\mathrm{z}}-\frac{\mathrm{r}_{33} \mathrm{n}_{\mathrm{z}}^{3} \mathrm{E}}{2} \\
\mathrm{n}_{\mathrm{x}}^{\prime}=\mathrm{n}_{\mathrm{x}}-\frac{\mathrm{r}_{13} \mathrm{n}_{\mathrm{x}}^{3} \mathrm{E}}{2}\end{array}$ & $\frac{2 \pi L}{\lambda}\left(n_{z}-n_{x}\right)+\frac{L}{d} \frac{\pi}{\lambda}\left(r_{13} n_{x}^{3}-r_{33} n_{z}^{3}\right) V_{\text {applied }}$ & $\frac{I_{0}}{2}\left(1+\sin \Gamma_{c} \cos \Gamma_{s}-\sin \Gamma_{c} \sin \Gamma_{s} \frac{L}{d} \frac{\pi}{\lambda}\left(\Gamma_{13} n_{x}^{3}-\Gamma_{33} n_{z}^{3}\right) V_{\text {applied }}\right.$ \\
\hline $\begin{array}{l}\text { L:[001] } \\
\mathrm{d}:[100]\end{array}$ & $\begin{array}{l}n_{y}^{\prime \prime}=n_{y}-\frac{r_{21} n_{y}^{3} E}{2} \\
n_{x}^{\prime \prime}=n_{x}-\frac{r_{11} n_{x}^{3} E}{2}\end{array}$ & $\frac{2 \pi L}{\lambda}\left(n_{y}-n_{x}\right)+\frac{L}{d} \frac{\pi}{\lambda}\left(r_{11} n_{x}^{3}-r_{21} n_{y}^{3}\right) V_{\text {applied }}$ & $\frac{I_{o}}{2}\left(1+\sin \Gamma_{c} \cos \Gamma_{s}-\sin \Gamma_{c} \sin \Gamma_{s} \frac{L}{d} \frac{\pi}{\lambda}\left(\Gamma_{11} n_{x}^{3}-\Gamma_{21} n_{y}^{3}\right) V_{\text {applied }}\right.$ \\
\hline
\end{tabular}


Table 5.2. Modified refractive indices, crystal retardations, and $\mathrm{I}_{\text {trx }}$ for two longitudinal YCOB modulators.

\begin{tabular}{|c|c|c|c|}
\hline $\begin{array}{l}\text { Crystal } \\
\text { Orientation } \\
\text { L:[xyz] } \\
\text { d:[xyz] }\end{array}$ & $\begin{array}{l}\mathrm{n}_{\mathrm{p}}^{\prime \prime} \\
\mathrm{n}_{\mathrm{q}}^{\prime \prime}\end{array}$ & $\Gamma_{\mathrm{xtal}}=\frac{2 \pi L}{\lambda}\left(\mathrm{n}_{\mathrm{p}}^{\prime \prime}-\mathrm{n}_{\mathrm{q}}^{\prime \prime}\right)$ & $\left.\frac{\mathrm{I}_{\mathrm{txx}}}{\left(\mathrm{V}_{\text {applied }}\right.}<\mathrm{V}_{\pi}\right)$ \\
\hline $\begin{array}{l}\mathrm{L}:[001] \\
\mathrm{d}:[001]\end{array}$ & $\begin{array}{l}n_{y}^{\prime \prime}=n_{y}-\frac{r_{23} n_{y}^{3} E}{2} \\
n_{x}^{\prime \prime}=n_{x}-\frac{r_{13} n_{x}^{3} E}{2}\end{array}$ & $\frac{2 \pi L}{\lambda}\left(n_{y}-n_{x}\right)+\frac{\pi}{\lambda}\left(r_{13} n_{x}^{3}-r_{23} n_{y}^{3}\right) V_{\text {applied }}$ & $\frac{I_{0}}{2}\left(1+\sin \Gamma_{c} \cos \Gamma_{s}-\sin \Gamma_{c} \sin \Gamma_{s} \frac{\pi}{\lambda}\left(r_{13} n_{x}^{3}-r_{23} n_{y}^{3}\right) V_{\text {applied }}\right)$ \\
\hline $\begin{array}{l}\mathrm{L}:[100] \\
\mathrm{d}:[100]\end{array}$ & $\begin{array}{l}\mathrm{n}_{\mathrm{z}}^{\prime \prime}=\mathrm{n}_{\mathrm{z}}-\frac{\mathrm{r}_{31} \mathrm{n}_{\mathrm{z}}^{3} \mathrm{E}}{2} \\
\mathrm{n}_{\mathrm{y}}^{\prime \prime}=\mathrm{n}_{\mathrm{y}}-\frac{\mathrm{r}_{21} \mathrm{n}_{\mathrm{y}}^{3} \mathrm{E}}{2}\end{array}$ & $\frac{2 \pi L}{\lambda}\left(n_{z}-n_{y}\right)+\frac{\pi}{\lambda}\left(r_{21} n_{y}^{3}-r_{31} n_{z}^{3}\right) N_{\text {applied }}$ & $\frac{I_{o}}{2}\left(1+\sin \Gamma_{c} \cos \Gamma_{s}-\sin \Gamma_{c} \sin \Gamma_{s} \frac{\pi}{\lambda}\left(\Gamma_{21} n_{y}^{3}-\Gamma_{31} n_{z}^{3}\right) V_{\text {applied }}\right)$ \\
\hline
\end{tabular}


Table 5.3. Modified refractive indices, crystal retardations, and $I_{t r x}$ for the KDP and $K D * P$ modulators.

\begin{tabular}{|c|c|c|c|}
\hline $\begin{array}{l}\text { Crystal } \\
\text { Orientation } \\
\text { L:[xyz] } \\
\text { d:[xyz] }\end{array}$ & $\begin{array}{l}\mathrm{n}_{\mathrm{p}}^{\prime \prime} \\
\mathrm{n}_{\mathrm{q}}^{\prime \prime}\end{array}$ & $\Gamma_{\mathrm{xtal}}=\frac{2 \pi L}{\lambda}\left(\mathrm{n}_{\mathrm{p}}^{\prime \prime}-\mathrm{n}_{\mathrm{q}}^{\prime \prime}\right)$ & $I_{t r x}$ \\
\hline $\begin{array}{c}\text { KDP } \\
\mathrm{L}:[110] \\
\mathrm{d}:[001]\end{array}$ & $\begin{array}{c}\mathrm{n}_{\mathrm{x}}^{\prime \prime}=\mathrm{n}_{\mathrm{o}}-\frac{\mathrm{n}_{\mathrm{o}}^{3} \mathrm{r}_{63} \mathrm{E}}{2} \\
\mathrm{n}_{\mathrm{z}}^{\prime \prime}=\mathrm{n}_{\mathrm{e}}\end{array}$ & $\frac{2 \pi L}{\lambda}\left(n_{0}-n_{e}\right)-\frac{L}{d} \frac{\pi}{\lambda} n_{o}^{3} r_{63} V_{\text {applied }}$ & $\frac{I_{o}}{2}\left(1+\sin \Gamma_{c} \cos \left(\Gamma_{s}-\frac{L}{d} \frac{\pi}{\lambda} n_{o}^{3} r_{63} V_{\text {applied }}\right)\right)$ \\
\hline $\begin{array}{c}\mathrm{KD} * \mathrm{P} \\
\mathrm{L}:[001] \\
\mathrm{d}:[001]\end{array}$ & $\begin{array}{l}\mathrm{n}_{\mathrm{y}}^{\prime \prime}=\mathrm{n}_{\mathrm{o}}+\frac{\mathrm{n}_{0}^{3} \mathrm{r}_{63} \mathrm{E}}{2} \\
\mathrm{n}_{\mathrm{x}}^{\prime \prime}=\mathrm{n}_{\mathrm{o}}-\frac{\mathrm{n}_{\mathrm{o}}^{3} \mathrm{r}_{63} \mathrm{E}}{2}\end{array}$ & $\frac{2 \pi \mathrm{n}_{0}^{3} \mathrm{r}_{63} \mathrm{~V}_{\text {applied }}}{\lambda}$ & $\frac{I_{0}}{2}\left(1+\sin \left(\Gamma_{c}+\frac{2 \pi}{\lambda} n_{o}^{3} r_{63} V_{\text {applied }}\right)\right)$ \\
\hline
\end{tabular}


in each experiment. The laser employed was a $14 \mathrm{~mW}$ polarized $\mathrm{CW}$ HeNe laser operating at $632.8 \mathrm{~nm}$. Before each of the $\mathrm{AC}$ experiments with the $\mathrm{KD} * \mathrm{P}$ crystal, the compensator was set to impart a retardation of $\Gamma_{c}=0$ and the crystal was aligned perpendicular to the beam using back reflections. Before each of the $\mathrm{AC}$ experiments with either the YCOB or KDP crystals, the compensator was set to $\Gamma_{c}=\pi / 2$. The crystal was then placed on the adjustable mount and tuned in angle such that maximum transmitted irradiance (i.e. $\Gamma_{s}=m \pi / 2$ where $m$ is an odd integer) was obtained through the analyzer as measured by the lock-in amplifier for a $5 \mathrm{kHz}, 20 \mathrm{~V}_{\text {peak-peak }}$ applied voltage. By changing the angle of the crystal $\left(\Delta \theta \leq 2.2^{\circ}\right)$, we effectively caused a small change in the value of $\mathrm{L}(\$ 14 \mu \mathrm{m})$ and therefore the retardation specified by $\Gamma_{s}$ was made to cycle through an odd multiple of $\pi / 2$. Only a small change in $L$ is needed to cause $\Gamma_{s}$ to cycle through a multiple of $\pi / 2$ because $L$ multiplies a term in $\Gamma_{s}$ that is large (i.e. $-1.1 \times 10^{3} \mathrm{radians} / \mathrm{cm}$ ).

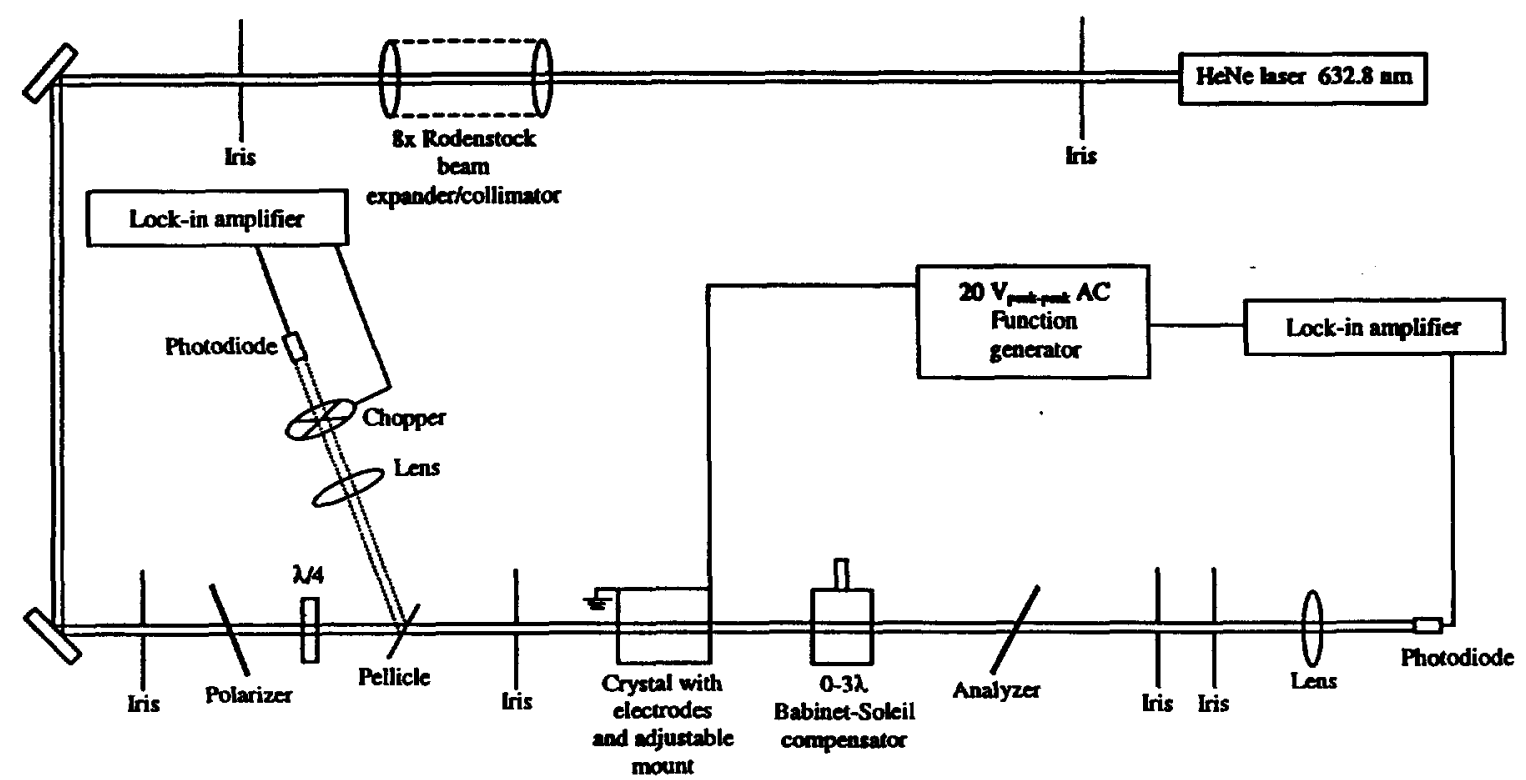

Figure 5.1. Experimental setup used for the AC electro-optic measurements. 
During the AC experiments, a $20 \mathrm{~V}_{\text {peak-peak, }} 5 \mathrm{kHz}$ sine wave from a Hewlett-Packard model 3325A function generator was applied to the crystals. The compensator was used to vary the transmitted irradiance while the fixed $\mathrm{AC}$ voltage was applied to the modulator (crystal). Adjusting the compensator allowed clear detection of the peak in the modulated irradiance. The compensator was adjusted in discrete steps through a total retardation of $6 \pi$ while the transmitted irradiance was measured using a Si photodiode and a Stanford Research Systems model SR830 DSP lock-in amplifier. Note that in the AC experiments, the irradiance that was measured by the lock-in amplifier was only that part of the total transmitted irradiance that was time varying or modulated at $5 \mathrm{kHz}$ (i.e. only the term in $I_{t r x}$ that depends on $V_{\text {applied }}$. Also, since we only measured the magnitude of the modulation with the lock-in amplifier and paid no attention to the phase, we cannot determine the sign of the $\left(\sin \Gamma_{s}\right)\left(r_{e f f}\right)$ term in the expressions for $I_{t r x}$. (For instance, with $\Gamma_{c}=\pi / 2, \Gamma_{s}$ could have equaled either $\pi / 2$ or $3 \pi / 2$ while $\Gamma_{\text {eff }}$ could have equaled either $+\left|r_{\text {eff }}\right|$ or $-\mid r_{\text {eff }} d$ and in either case we still would have measured a maximum magnitude for the modulation.) Consequently, we will only be able to determine the absolute value of $r_{\text {eff }}$ from our AC data.

The experimental setup used for the DC measurements is shown in Fig. 5.2. In the DC experiments, $0-3 \mathrm{kV}$ was applied to the crystals in discrete steps using a HewlettPackard model 6516A DC power supply. The total transmitted irradiance as a function of the applied DC voltage was measured by chopping the laser beam at $3.75 \mathrm{kHz}$ and detecting the transmitted irradiance using a photodiode and a lock-in amplifier. The input irradiance $\left(\mathrm{I}_{0}\right)$ was measured simultaneously from a beam pick-off using a photodiode and a lock-in amplifier and was used to correct the transmitted irradiance data for 


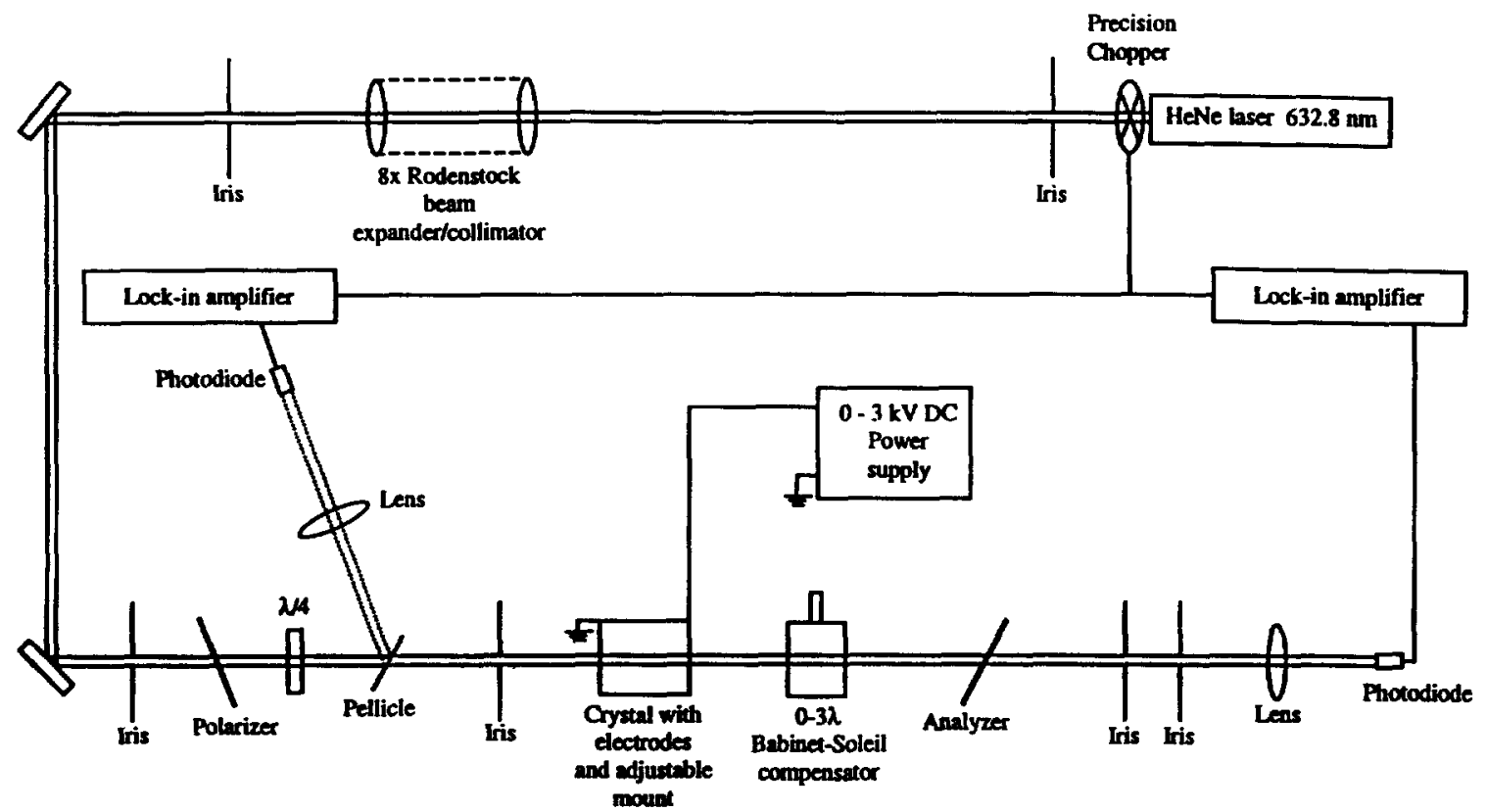

Figure 5.2. Experimental setup used for the DC electro-optic measurements.

variations in the input irradiance.

Before beginning the DC experiments, the crystal was tuned to maximum transmitted irradiance in the AC experiment as described above and then the experimental setup was reconfigured to that shown in Fig. 5.2. The compensator remained at its initial setting of either 0 or $\pi / 2$ throughout the $\mathrm{DC}$ experiments. The $\mathrm{DC}$ voltage was applied to the crystal and then increased in a few steps while the transmitted irradiance was observed to either increase or decrease. If the transmitted irradiance decreased, the experiment was reconfigured back to the AC arrangement and the crystal's angle was changed until $\Gamma_{s}$ cycled to the next multiple of $\pi / 2$. Since we did not determine the sign of the $\sin \Gamma_{\mathrm{s}}$ term in $I_{t x x}$ and we did not note the sign of the DC applied voltage, we will only be able to determine the magnitude of $r_{\text {eff }}$ from our DC measurements.

The AC and DC experiments were repeated several times with each crystal to verify the reproducibility of the data. The AC data for each crystal varied by approximately 
$15 \%$ between the data sets. The DC data for each crystal varied by approximately $10 \%$ between the data sets.

\subsection{Experimental results}

We measured the transmitted irradiance through a $\mathrm{KDP}, \mathrm{KD} * \mathrm{P}$, and six different YCOB modulators while an AC or a DC voltage was applied. Only the data collected on three of the six YCOB modulators is shown in Figs. 5.3 and 5.4. The other three configurations had very small relative electro-optic responses. The dashed lines through the data in Fig. 5.3 are only to aid the eye. The solid lines through the data in Fig. 5.4 are the numerical fits of the corresponding equations given in Tables $5.1-5.3$.

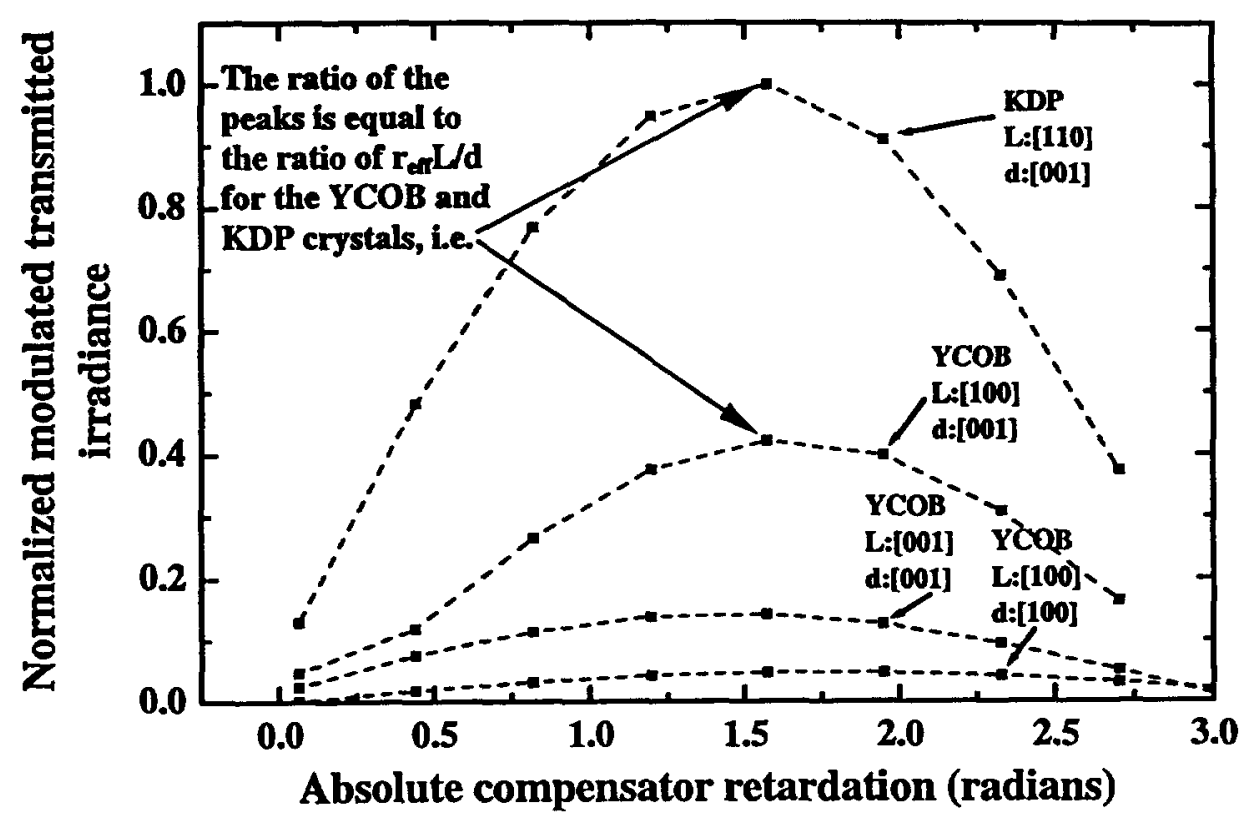

Figure 5.3. Normalized modulated irradiance through the KDP and YCOB modulators as a function of the compensator retardation for a $20 \mathrm{~V}, 5 \mathrm{kHz}(\mathrm{AC})$ applied voltage.

The AC data shown in Fig. 5.3 was corrected in several ways. The input beam irradiance from the HeNe laser was noticed to vary by approximately $\pm 5 \%$ between the data sets and so the data sets were first normalized to an equivalent input beam 


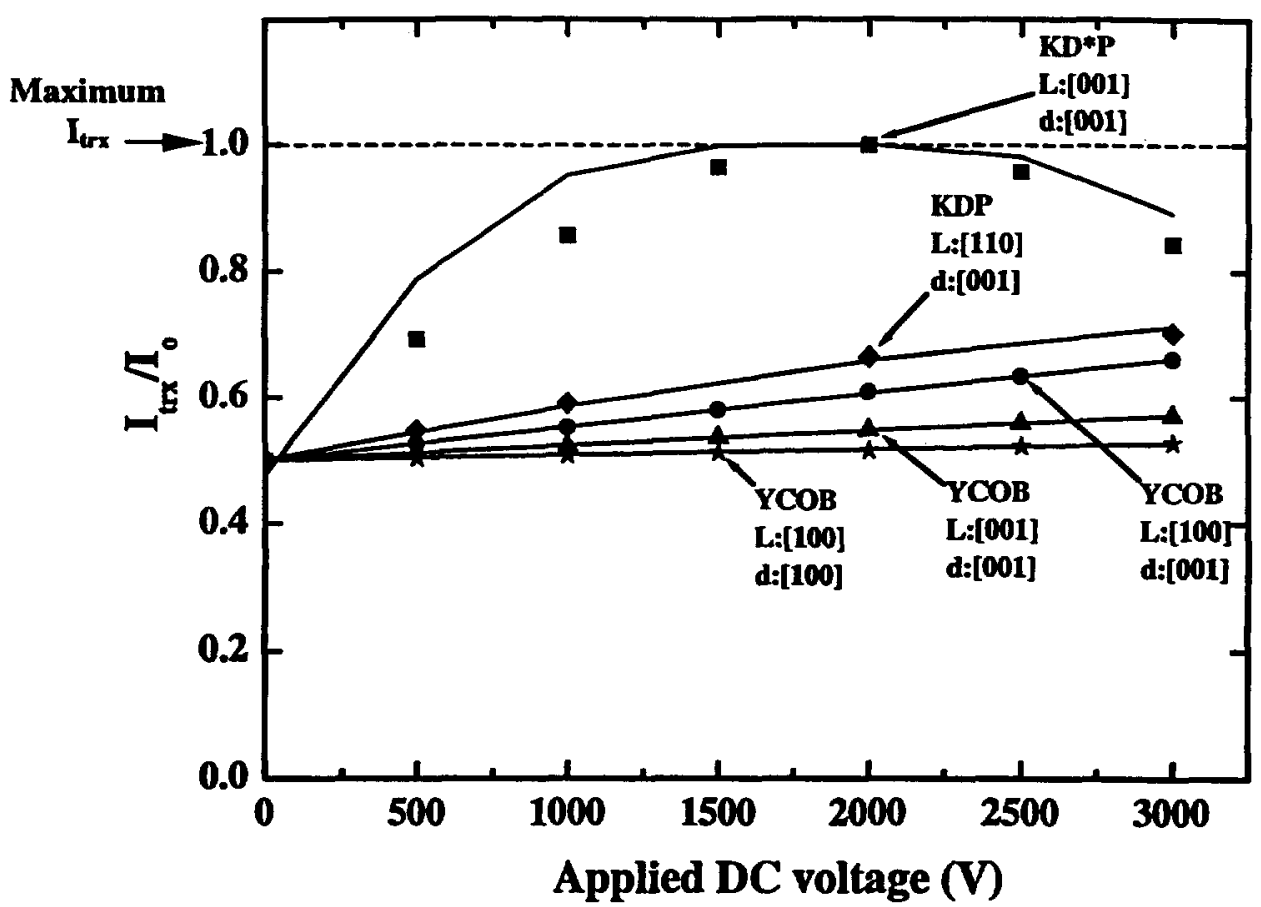

Figure 5.4. Transmission through the $\mathrm{KD} * \mathrm{P}, \mathrm{KDP}$, and $\mathrm{YCOB}$ modulators as a function of the applied DC voltage. Note that a transmission of 1 implies $100 \%$ transmission through the modulators.

irradiance. Second, the refractive index of YCOB differs from that of KDP and KD*P by approximately $13 \%$ and so the transmitted irradiances were adjusted to account for the differences in the Fresnel reflections. Third, all the data sets in Fig. 5.3 were normalized by the peak modulated irradiance produced by the KDP modulator. Therefore the ratio of the peak irradiances produced by the YCOB modulators to that of the KDP modulator is equal to the ratio of $r_{e f f} L / d$ for the YCOB and KDP crystals. Fourth, the transmitted irradiances in the longitudinal experiments were also adjusted to account for the measured differences in the transmission $(-20 \%)$ of the semi-transparent gold electrodes that had been coated onto the different crystals.

The DC data in Fig. 5.4 is shown normalized by the measured input beam irradiance $\left(I_{0}\right)$. The DC data was also corrected for the calculated Fresnel losses of the YCOB 
crystals. Note that because a quarter-wave plate was used in the modulators the percent transmission for no applied voltage is $50 \%$ [9]. Also, the data for $\mathrm{KD} * \mathrm{P}$ reached $100 \%$ transmission because $\mathrm{V}_{\mathbb{n}} / 2$ at $632.8 \mathrm{~nm}$ for $\mathrm{KD}^{*} \mathrm{P}$ is approximately $1950 \mathrm{~V}$.

\subsection{Effective electro-optic coefficients and half-wave voltages}

Table 5.4 displays the $\mathrm{AC}$ and $\mathrm{DC}$ determined values for the effective electro-optic coefficients. The experimental configurations for the crystals within the modulators are as indicated. $\mathrm{V}_{\text {applied }}$ is the applied DC or low frequency $A C$ voltage, $\vec{k}$ is the wavevector for the laser beam, $r_{e f f}$ is the relevant effective electro-optic coefficient, and $V_{\star}(1064 \mathrm{~nm})$ is the calculated half-wave voltage at $1064 \mathrm{~nm}$ assuming $\mathrm{L}=\mathrm{d}$. For the $\mathrm{YCOB}$ and $\mathrm{BBO}$ crystals, $V_{\pi}(1064 \mathrm{~nm})$ was calculated using the $\mathrm{AC}$ values for $\mathrm{r}_{\text {eff }}$ and for the KDP and KD*P crystals it was calculated using the DC values for $\mathrm{r}_{\mathrm{eff}}$. The equations in Tables 5.1 - 5.3 and the data in Figs. 5.3 and 5.4 were used to calculate the $r_{\text {eff }}$ values that are shown. Note that the data that was used to calculate the $r_{\text {eff }}$ values for YCOB in the $(\mathrm{L}:[010] \mathrm{d}:[100]),(\mathrm{L}:[010] \mathrm{d}:[100])$, and (L:[010] d:[100]) configurations in Table 5.4 was measured but was not shown in the figures. The data for BBO is from the cited reference.

The AC values for the $r_{\text {eff }}$ 's in Table 5.4 were obtained by comparing the peak modulated irradiances for the YCOB modulators to the peak modulated irradiance for the KDP modulator and using $r_{\text {eff }}=35.8 \mathrm{pm} / \mathrm{V}$ for KDP. We calibrated our AC experiment by comparing KDP to KD*P from which we determined that the ratio of the $r_{63}$ 's for KDP and $K \mathrm{KD}^{*} \mathrm{P}$ was 0.423 . This agrees within $8 \%$ of the ratio of values found in Ref. [17].

The DC values for the $r_{\text {eff }} s$ in Table 5.4 were calculated from the slope of the numerical fit of the appropriate equations in Tables $5.1-5.3$ to the DC data. (Note that 
in the expressions for $I_{t x x}$ in Tables 5.1 and 5.2, $I_{t r x}$ is linearly proportional to the applied voltage with the constant of proportionality containing $r_{\text {eff. }}$ Therefore from the slope parameter of the numerical fit we can extract the magnitude of $r_{\text {eff. }}$ We do not determine any information about the sign of $r_{\text {eff }}$ from the slopes for the reasons discussed in section 5.2) In general, the numerical fits were good as shown in Fig. 5.4. However, the fitting parameters varied by approximately $10 \%$ amongst the data sets for a particular crystal.

We calibrated our DC experiment by determining $r_{\text {eff }}$ for KDP in a transverse configuration and $r_{\text {eff }}$ for KD*P in a longitudinal configuration. From the slopes of the fitted lines to the data for KDP and KD*P, we determined $r_{\text {eff }}=n_{0}{ }^{3}{ }_{63}=35.8 \pm 4.5 \mathrm{pm} / \mathrm{V}$ for $\mathrm{KDP}$ and $\mathrm{r}_{\mathrm{eff}}=2 \mathrm{n}_{\mathrm{o}}{ }^{3} \mathrm{r}_{63}=159.9 \pm 9.5 \mathrm{pm} / \mathrm{V}$ for $\mathrm{KD} * \mathrm{P}$. $\mathrm{r}_{63}$ values were then calculated

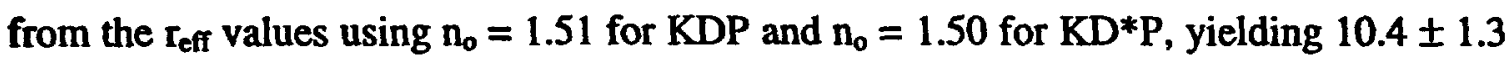
$\mathrm{pm} / \mathrm{V}$ and $23.7 \pm 1.4 \mathrm{pm} / \mathrm{V}$ for $\mathrm{KDP}$ and $\mathrm{KD} * \mathrm{P}$, respectively. The $\mathrm{r}_{63}$ values determined here for KDP and KD*P agree with that of Ref. [17]. In general, we see good agreement between the $A C$ and $D C$ values for the $r_{\text {eff }}$ 's in Table 5.4 which would suggest only a small $(<15 \%)$ or zero photo-elastic $[6,11]$ contribution to the $r_{\text {eff }}$ values determined in the $5 \mathrm{kHz}$ AC experiments.

From the $r_{\text {eff }}$ values for YCOB in Table 5.4, we see that the maximum $r_{\text {eff }}$ in the transverse configurations is $10.81 \pm 1.46 \mathrm{pm} / \mathrm{V}$ and the maximum $\mathrm{r}_{\text {eff }}$ in the longitudinal configurations is $10.7 \pm 1.0 \mathrm{pm} / \mathrm{V}$. The corresponding half-wave voltage calculated at $1064 \mathrm{~nm}$ with $\mathrm{L}=\mathrm{d}$ for either of these two large coefficients is $99 \mathrm{kV}$. A half-wave voltage of $99 \mathrm{kV}$ is a little more than twice that for an equivalent length of $\mathrm{BBO}$ and substantially more than the half-wave voltage for either $\mathrm{KDP}$ or $\mathrm{KD} * \mathrm{P}$. The value for $\mathrm{r}_{\mathrm{eff}}$ for the other longitudinal configuration with $\mathrm{YCOB}$ is $\sim 3 \mathrm{pm} / \mathrm{V}$ which implies an 
Table 5.4. Experimentally determined effective electro-optic coefficients and calculated half-wave voltages.

\begin{tabular}{|c|c|c|c|c|c|c|}
\hline Crystal & $\begin{array}{l}\text { EO experiment } \\
\overrightarrow{\mathrm{k}} \text { and } \mathrm{V}_{\text {applied }} \\
\text { configurations }\end{array}$ & $\begin{array}{c}\text { Propagation length (L) and } \\
\text { Applied voltage length (d) } \\
(\mathrm{mm})\end{array}$ & $r_{e f f}$ & $\begin{array}{c}\mathrm{I}_{\text {eff }}(632.8 \mathrm{~nm}) \\
(5 \mathrm{kHz} \mathrm{AC} \text { values }) \\
(\mathrm{pm} / \mathrm{V})\end{array}$ & $\begin{array}{c}\mathrm{I}_{\text {eff }}(632.8 \mathrm{~nm}) \\
\text { (DC values) } \\
(\mathrm{pm} / \mathrm{V}) \\
\end{array}$ & $\begin{array}{l}\mathrm{V}_{\pi}(1064 \mathrm{~nm}) \\
\quad(L=\mathrm{d})\end{array}$ \\
\hline $\mathrm{YCOB}$ & $\begin{array}{l}\mathrm{L}:[100] \\
\mathrm{d}:[001] \\
\end{array}$ & $\begin{array}{l}\mathrm{L}=17.81 \\
\mathrm{~d}=6.48\end{array}$ & $\left(r_{33} n_{z}^{3}-r_{23} n_{y}{ }^{3}\right)$ & $10.81 \pm 1.46$ & $8.79 \pm 1.46$ & $98 \mathrm{kV}$ \\
\hline $\mathrm{YCOB}$ & $\begin{array}{l}\mathrm{L}:[010] \\
\mathrm{d}:[100]\end{array}$ & $\begin{array}{l}\mathrm{L}=14.26 \\
\mathrm{~d}=3.94\end{array}$ & $\left(r_{31} n_{z}^{3}-r_{11} n_{x}^{3}\right)$ & $\leq \mathbf{0 . 2 0}$ & $\leq 0.27$ & - \\
\hline $\mathrm{YCOB}$ & $\begin{array}{l}\mathrm{L}:[010] \\
\mathrm{d}:[001]\end{array}$ & $\begin{array}{l}L=14.26 \\
d=7.36\end{array}$ & $\left(r_{33} n_{2}^{3}-r_{13} n_{x}^{3}\right)$ & $\leq \mathbf{0 . 5 2}$ & $\leq \mathbf{0 . 5 5}$ & - \\
\hline YCOB & $\begin{array}{l}\mathrm{L}:[001] \\
\mathrm{d}:[100]\end{array}$ & $\begin{array}{l}L=7.98 \\
d=9.86\end{array}$ & $\left(r_{21} n_{y}{ }^{3}-r_{11} n_{x}{ }^{3}\right)$ & $\leq 0.82$ & $\leq 1.8$ & - \\
\hline YCOB & $\begin{array}{l}L:[100] \\
d:[100]\end{array}$ & $\begin{array}{l}L=9.60 \\
d=9.60\end{array}$ & $\left(r_{31} n_{z}{ }^{3}-r_{21} n_{y}{ }^{3}\right)$ & $3.43 \pm 0.49$ & $3.4 \pm 0.4$ & $313 \mathrm{kV}$ \\
\hline YCOB & $\begin{array}{l}\mathrm{L}:[001] \\
\mathrm{d}:[001]\end{array}$ & $\begin{array}{l}L=6.08 \\
d=6.08\end{array}$ & $\left(r_{23} n_{y}^{3}-r_{13} n_{x}^{3}\right)$ & $10.3 \pm 1.6$ & $10.7 \pm 1.0$ & $99 \mathrm{kV}$ \\
\hline $\mathrm{KDP}$ & $\begin{array}{l}\mathrm{L}:[110] \\
\mathrm{d}:[001]\end{array}$ & $\begin{array}{l}L=20.33 \\
d=10.05\end{array}$ & $n_{0}^{3} r_{63}$ & \multirow{2}{*}{$\frac{r_{\mathrm{ef}}^{\mathrm{KDP}}}{\mathrm{r}_{\mathrm{eff}}^{\mathrm{KD} P}}=0.216$} & $35.8 \pm 4.5$ & $30 \mathrm{kV}$ \\
\hline $\mathrm{KD} * \mathrm{P}$ & $\begin{array}{l}\mathrm{L}:[001] \\
\mathrm{d}:[001]\end{array}$ & $\begin{array}{l}L=17.58 \\
d=17.58\end{array}$ & $2 n_{0}^{3} r_{63}$ & & $159.9 \pm 9.5$ & $6.7 \mathrm{kV}$ \\
\hline $\mathrm{BBO}[13]$ & $\begin{array}{l}\text { L:[001] } \\
\text { d:[010] }\end{array}$ & $\begin{array}{l}L=5.05 \\
d=8.12\end{array}$ & $2 n_{0}{ }^{3} r_{22}$ & $23.3^{a}$ & $19.6^{\mathrm{a}}$ & $46 \mathrm{kV}$ \\
\hline
\end{tabular}

"In the cited work, the $r_{22}$ coefficient was measured at $\lambda=514.5 \mathrm{~nm}$ and a value of $n_{0}=1.67$ was used here to calculate the values shown. 
impractical half-wave voltage of $313 \mathrm{kV}$.

Examination of the remaining transverse results for $\mathrm{YCOB}$ reveals $\mathrm{r}_{\mathrm{eff}}$ coefficients that have upper bounds $\leq 2 \mathrm{pm} / V$. The results for the coefficients $\left(r_{23} n_{y}{ }^{3}-r_{13} n_{x}{ }^{3}\right)$ and $\left(r_{33} n_{z}{ }^{3}-r_{23} n_{y}{ }^{3}\right)$ suggest that $r_{13}$ and $r_{33}$ are either small, equal in magnitude, or both. Various combinations of the coefficients for YCOB in Table 5.4 were examined to determine if the values were consistent. Nothing meaningful or consistent was deduced in this way for the different coefficients in Table 5.4. This is most likely due to the relatively large error bars $(-15 \%)$ on the larger coefficients and that only upper bound estimates for the smaller coefficients $(<2 \mathrm{pm} / \mathrm{V})$ could be determined because of the low signal-to-noise ratio in the data.

\subsection{Summary and conclusions}

We have characterized the effective linear electro-optic coefficients along dielectric directions in YCOB for four transverse and two longitudinal configurations. We find a maximum $r_{\text {eff }}$ of $10.81 \pm 1.46 \mathrm{pm} / \mathrm{V}$ for the transverse configurations and a maximum $r_{\text {eff }}$ of $10.7 \pm 1.0 \mathrm{pm} / \mathrm{V}$ for the longitudinal configurations. The effective electro-optic coefficients for the other transverse and longitudinal configurations were measured to be $<4 \mathrm{pm} / \mathrm{V}$. We see that our estimate of the effective electro-optic coefficient $\mathrm{r}_{23} \mathrm{n}^{3}$ of $\sim 14$ $\mathrm{pm} / \mathrm{V}$ is consistent (i.e. within a factor of two) with the measured $\mathrm{r}_{\text {eff }}$ values that contain the $r_{23}$ coefficient (i.e. $r_{\text {eff }}=r_{33} n_{z}{ }^{3}-r_{23} n_{y}{ }^{3}=10.81 \pm 1.46 \mathrm{pm} / V$ ). Also if we estimate the effective electro-optic coefficient $\mathbf{r}_{21} \mathrm{n}^{3}$ in a similar manner we find its value to be -2 $\mathrm{pm} / \mathrm{V}$ which is consistent (i.e. within a factor of two) with the measured $r_{\text {eff }}$ values that contain the $r_{12}$ coefficient (i.e. $r_{\text {eff }}=r_{31} n_{z}{ }^{3}-r_{21} n_{y}{ }^{3}=3.43 \pm 0.49 p m / V$ ). 
Half-wave voltages of $\sim 100 \mathrm{kV}$ were found for YCOB crystals with 1:1 aspect ratios (i.e. $\mathrm{L}=\mathrm{d}$ ). Electro-optic crystals in configurations with half-wave voltages $<10 \mathrm{kV}$ are preferable. Therefore single crystal electro-optic applications involving YCOB maybe rare in the near future. However, YCOB's ease of growth and handling makes large aperture, multi-plate longitudinal configurations feasible. The advantage of using multiple plates is that the required half-wave voltage would be much less than $99 \mathrm{kV}$. Another useful configuration would be to employ a pair of identical YCOB crystals each with a 5:1 (L:d) aspect ratio separated by a $90^{\circ}$ rotator. One advantage of this architecture is that it compensates for the crystals' static and thermally induced birefringence. Another advantage is that because of the 5:1 aspect ratio of the YCOB crystals, the half-wave voltage is $<10 \mathrm{kV}$. YCOB may therefore have potential application as a large aperture $1 \mu \mathrm{m} \mathrm{Q}$-switch. 


\section{References for Chapter 5}

[1] R. Norrestam, M. Nygren, and J.-O. Bovin, Chem. Mater. 4, 737 (1992)

[2] M. Iwai, T. Kobayashi, H. Furuya, Y. Mori, and T. Sasaki, Jpn. J. Appl. Phys. 36, L276 (1997)

[3] Q. Ye and B. H. T. Chai, J. Cryst. Growth 197, 228 (1999)

[4] Y. Mori and T. Sasaki, Bull. Mater. Sci. 22, 399 (1999)

[5] H. Hellwig, J. Liebertz, and L. Bohaty, J. Appl. Phys. 88, 240 (2000)

[6] J. F. Nye, Physical Properties of Crystals, 1985 ed. (Clarendon Press, Oxford, 1985)

[7] D. Eimerl, L. Davis, S. Velsko, E. K. Graham, and A. Zalkin, J. Appl. Phys. 62, 1968 (1987)

[8] F. Zernike and J. E. Midwinter, Applied Nonlinear Optics, (John Wiley and Sons, Inc., New York, 1973)

[9] A. Yariv and P. Yeh, Optical Waves in Crystals - Propagation and Control of Laser Radiation, (John Wiley and Sons, Inc., New York, 1984)

[10] C. A. Ebbers, J. Opt. Soc. Am. B 12, 1012 (1995)

[11] R. O'B. Carpenter, J. Opt. Soc. Am. 40, 225 (1950)

[12] G. E. Peterson, A. A. Ballman, P. V. Lenzo, and P. M. Bridenbaugh, Appl. Phys. Lett. 5, 62 (1964)

[13] C. A. Ebbers, Appl. Phys. Lett. 52, 1948 (1988)

[14] V. G. Dmitriev, et al., Handbook of Nonlinear Optical Crystals, $3^{\text {rd }}$ ed. (SpringerVerlag, Berlin, 1999)

[15] J. J. Adams, C. A. Ebbers, K. I. Schaffers, and S. A. Payne, OSA Trends in Optics and Photonics Series Vol. 50, Advanced Solid State Lasers, C. Marshall, ed. (Optical Society of America, Washington, DC 2001), p. 615

[16] Cleveland Crystals, Inc. website, www.clevelandcrystals.com/KDP.shtml, (2002)

[17] Onuki, K., Uchida, N., and T. Saku, J. Opt. Soc. Am. 62, 1030 (1972) 


\section{Chapter 6}

\section{Optical spectroscopy of $\mathrm{Fe}^{2+}: \mathrm{ZnSe}$ crystals}

\subsection{Introduction}

Atmospheric remote sensing of gas pollutants or aerosol plumes and laser radar (LIDAR) involve transmitting a beam of laser light over large distances through the earth's atmosphere $(5-10 \mathrm{~km})$ [1]. These applications also typically require a certain tunability of the laser's wavelength [2]. The earth's atmosphere contains, among others, the gases $\mathrm{O}_{2}, \mathrm{CO}_{2}$, and water vapor. The presence of these constituents give rise to strong absorption bands in the earth's atmosphere throughout the $0.5-5 \mu \mathrm{m}$ wavelength range [3]. The effect of these absorption bands will cause nearly $100 \%$ attenuation of the laser beam for propagation through the earth's atmosphere of only a few hundred meters. There does exist however, "atmospheric transmission windows" in the $0.5-5 \mu \mathrm{m}$ range where there is nearly $100 \%$ transmission. Transmitters for remote sensing and LIDAR ideally need to operate at wavelengths that lie in these atmospheric transmission windows. Two broad atmospheric transmission windows exist from 2.0 to $2.5 \mu \mathrm{m}$ and from 3.4 to $4.2 \mu \mathrm{m}$ [3]. There are no commercial direct solid-state lasers available in these wavelength ranges and so optical parametric oscillators (OPO) are typically used for these applications. OPO's, though broadly tunable, incorporate nonlinear crystals and moderate power laser pump sources, which add complexity and bulk to the system. It would be desirable therefore, from a design point of view, to find gain materials that would allow the operation of direct solid-state lasers in these two wavelength "windows".

Much work has been done in the past 40 years investigating the optical properties of transition metal impurities in zinc-blende crystals [4-25]. In the 1960's and early 1970's, 
transition metal impurities (i.e. $\mathrm{Cr}^{2+}$ and $\mathrm{Fe}^{2+}$ ) in zinc-blende crystals were found to broadly luminesce in the $2-5 \mu \mathrm{m}$ range. $\mathrm{Fe}^{2+}$ impurities were originally characterized because of their luminescence "quenching" behavior at room temperature in the phosphors (based on II-VI compounds) used in early fluorescent displays [22]. The infrared luminescence of $\mathrm{Fe}^{2+}$ impurities in $\mathrm{ZnSe}\left(\mathrm{Fe}^{2+}: \mathrm{ZnSe}\right)$ was first reported by Haanstra [22] in 1967. He observed broad absorption and emission bands centered around $3.7 \mu \mathrm{m}$ at temperatures of $8 \mathrm{~K}$ and $18 \mathrm{~K}$, respectively. $\left(\mathrm{Fe}^{2+}: \mathrm{ZnSe}\right.$ only luminesces for temperatures below about $225 \mathrm{~K}$ because of thermally-activated nonradiative coupling to the vibrational modes of the $\mathrm{ZnSe}$ lattice.) About 16 years later, in $1983, \mathrm{Fe}^{2+}$ was first lased in $\mathrm{n}-\mathrm{InP}$ at $2 \mathrm{~K}$ by Klein et al. [23] who were studying photoluminescence and non-radiative transitions of $\mathrm{Fe}^{2+}$ impurities in $\mathrm{n}$-InP. Direct optical pumping at $580 \mathrm{~nm}$ was used to reach laser threshold at $3.5 \mu \mathrm{m}$. The laser had a threshold of about $90 \mu \mathrm{J} /$ pulse and an estimated tunability of $0.07 \mu \mathrm{m}$. DeLoach et al. in $1996[24,25]$ investigated the spectroscopic properties of primarily $\mathrm{Cr}^{2+}, \mathrm{Co}^{2+}$, and $\mathrm{Ni}^{2+}$ ions in $\mathrm{ZnTe}, \mathrm{ZnS}$, and $\mathrm{ZnSe}$ hosts. Their efforts were mainly focused on finding room temperature infrared laser materials for use in surgical lasers, which utilize the water absorption in human tissue at about $2.7 \mu \mathrm{m}$ for cutting and drilling. They identified $\mathrm{Cr}^{2+}: \mathrm{ZnS}, \mathrm{Cr}^{2+}: \mathrm{ZnTe}$, and $\mathrm{Cr}^{2+}: \mathrm{ZnSe}$ as strong candidates in the $2.0-2.8 \mu \mathrm{m}$ range. They also examined $\mathrm{Fe}^{2+}: \mathrm{ZnSe}$ and found a broad absorption band at $-2900 \mathrm{~nm}$ but no luminesce at room temperature which is in agreement with previous researchers. They reported luminescence lifetime data for $\mathrm{Fe}^{2+}: \mathrm{ZnSe}$ for temperatures between $100 \mathrm{~K}$ and $225 \mathrm{~K}$ but no absorption or emission spectra were presented.

Our interest in $\mathrm{Fe}^{2+}$ impurities in the zinc chalcogenides lies in the possibility for 
broadly tunable laser emission in the $4.0 \mu \mathrm{m}$ range. Based on previous work $[22,24]$, it can be estimated that a laser based on $\mathrm{Fe}^{2+}: \mathrm{ZnSe}$ would only operate at cryogenic temperatures but would have significant tunability from approximately $3.6 \mu \mathrm{m}-4.2 \mu \mathrm{m}$. The focus of this chapter is to accurately determine the absorption and emission wavelengths, absorption and emission cross-sections, and luminescence lifetimes for $\mathrm{Fe}^{2+}: \mathrm{ZnSe}$ in the $4.0 \mu \mathrm{m}$ range as a function of temperature. Quantitative knowledge of these properties will be used in the next chapter to predict $\mathrm{Fe}^{2+}: \mathrm{ZnSe}$ 's potential to exhibit laser oscillation.

In the first part of this chapter (section 6.2), the theoretical splitting of the ground and first excited state of a $\mathrm{Fe}^{2+}$ free-ion by an external tetrahedral crystal field and spin-orbit coupling is discussed. This corresponds to the static situation describing $\mathrm{Fe}^{2+}$ impurity ions in a $\mathrm{ZnSe}$ lattice. The effects of the $\mathrm{Fe}^{2+}$ ions being coupled to a vibrating crystal lattice are then discussed in terms of a configuration coordinate diagram. The temperature dependent absorption, emission, and lifetime data measured for $\mathrm{Fe}^{2+}: \mathrm{ZnSe}$ are presented in section 6.3. In section 6.4 , the absorption and emission data are analyzed and used to construct an accurate energy level diagram for the ground and first excited states for $\mathrm{Fe}^{2+}: \mathrm{ZnSe}$ that includes both electronic and vibrational levels. Section 6.5 summarizes the major results.

\subsection{Theoretical background}

In the next several sections, a theoretical description for the energy level structure for $\mathrm{Fe}^{2+}$ impurities in a static and vibrating $\mathrm{ZnSe}$ lattice is developed. 


\subsubsection{Energy states for $\mathrm{Fe}^{2+}: \mathrm{ZnSe}$}

Since $\mathrm{Fe}^{2+}$ and $\mathrm{Zn}^{2+}$ have nearly equal ionic radii, $\mathrm{Fe}^{2+}$ will preferentially substitute for $\mathrm{Zn}^{2+}$ in the $\mathrm{ZnSe}$ lattice. The $\mathrm{Fe}^{2+}$ ions in the $\mathrm{ZnSe}$ lattice have as nearest neighbors four tetrahedrally coordinated $\mathrm{Se}^{2-}$ ions. Therefore the crystal field that $\mathrm{Fe}^{2+}$ will "experience" has tetrahedral symmetry. $\mathrm{Fe}^{2+}$ has six valence electrons, all which reside in its $3 \mathrm{~d}$ orbitals. The $3 \mathrm{~d}$ orbitals are radially the farthest occupied orbitals from the $\mathrm{Fe}^{2+}$ nucleus and therefore $\mathrm{Fe}^{2+}$ bonds to the $\mathrm{ZnSe}$ lattice primarily through these orbitals. As a result, the effect of the external crystal field on the valence electrons of $\mathrm{Fe}^{2+}$ is a stronger interaction than their spin-orbit coupling. For the moment, we will assume that the $\mathrm{ZnSe}$ lattice is motionless and that $\mathrm{Fe}^{2+}$ sees only a static tetrahedral crystal field.

We will now postulate a Hamiltonian (Eqn. (6.1)) that will describe the $\mathrm{Fe}^{2+}$ ions in the $\mathrm{ZnSe}$ lattice sufficient for our purposes. The metals in the first transition series of the periodic table can be described by Russell-Saunders coupling since the spin-orbit interaction is weak compared to the Coulomb interaction between the electrons. In Russell-Saunders coupling, $\mathrm{L}$ and $\mathrm{S}$ are treated as good quantum numbers, and the spinorbit interaction Hamiltonian has the form $\lambda(\overrightarrow{\mathrm{L}} \cdot \overrightarrow{\mathrm{S}})$ [26]. The terms in Eqn. (6.1) are arranged in order of decreasing interaction strength. Eqn. (6.1) along with appropriate orbital wavefunctions (that will not be discussed here) can be used to calculate the energy

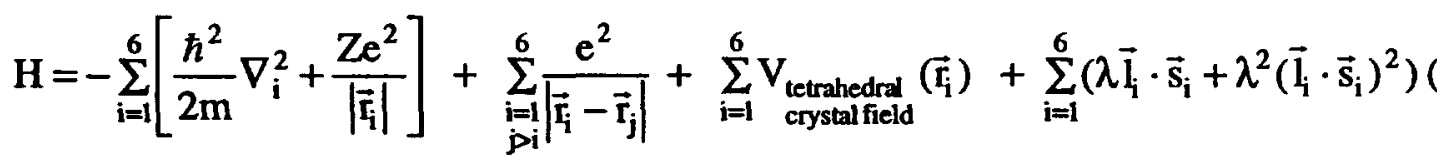

$$
\begin{aligned}
& \text { Coulombic }
\end{aligned}
$$


spectrum for $\mathrm{Fe}^{2+}$ impurities in $\mathrm{ZnSe}$. Detailed calculations for the energy levels using this Hamiltonian can be found in Refs. [27-29] and will be summarized next.

The ZnSe crystal field splits the $\mathrm{Fe}^{2+}$ free-ion atomic terms according to the TanabeSugano diagrams [28]. These diagrams are derived using the first three terms of Eqn. (6.1). The Tanabe-Sugano diagrams are expressed in terms of the multi-electron free-ion atomic terms (energy states), the crystal field split terms, the crystal field strength parameter $(\Delta=10 \mathrm{Dq})$, the energy $(E)$ of each crystal field split term, and two of the Racah [28] parameters (B and C). Each of these quantities will now be discussed in the context of $\mathrm{Fe}^{2+}: \mathrm{ZnSe}$.

The multi-electron atomic terms or energy states for a free $\mathrm{Fe}^{2+}$ ion can be determined by first tabulating the allowed combinations for the z-components of the individual orbital $\left(m_{l}\right)$ and spin $\left(m_{s}\right)$ angular momenta for each of its six $d$ electrons. Secondly, the individual $m_{l}$ 's and $m_{s}$ 's for each allowed combination can then be summed to determine the allowed values for the $\mathrm{z}$-components of the total orbital (i.e. $\mathrm{m}_{\mathrm{L}}=\Sigma \mathrm{m}_{\mathrm{l}}$ ) and spin (i.e. $m_{S}=\sum m_{s}$ ) angular momenta. If we then use Eqns. (6.2) and (6.3) to group the values of

$$
\begin{aligned}
& \mathrm{m}_{\mathrm{L}}=-\mathrm{L},-\mathrm{L}+1, \ldots 0 \ldots, \mathrm{L}-1,+\mathrm{L} \\
& \mathrm{m}_{\mathrm{S}}=-\mathrm{S},-\mathrm{S}+1, \ldots 0 \ldots, \mathrm{S}-1,+\mathrm{S}
\end{aligned}
$$

$m_{L}$ and $m_{S}$ we can determine the $L$ and $S$ combinations that arise. The $L$ and $S$ combinations or states are written as ${ }^{2 S+1} \mathrm{~L} .2 S+1$ is the spin multiplicity of the term. $\mathrm{L}$ is the letter designation of the total angular momentum of the term with the total angular momentum given by $\sqrt{\mathrm{L}(\mathrm{L}+1)} \hbar$. For historical reasons, letter symbols are given to the terms according to the value of $L$ as designated by: 


$$
\mathrm{L} \text { value }=\quad \begin{array}{lllllll}
0 & 1 & 2 & 3 & 4 & 5 & 6 \ldots
\end{array}
$$

$\begin{array}{lllllllll}\text { Term symbol: } & \text { S } & \text { P } & \text { D } & \text { F } & \text { G } & \text { H } & \text { I } \ldots\end{array}$

Table 6.1 lists the terms in order of increasing energy for a $\mathrm{Fe}^{2+}$ free-ion. The term with lowest energy can be determined using Hund's first rule that states that the term with the highest spin multiplicity (i.e. ${ }^{5} \mathrm{D}$ ) will be lowest in energy. The relative energies

Table 6.1. Energy states for a $\mathrm{Fe}^{2+}\left(\mathrm{d}^{6}\right)$ free-ion in the Russell-Saunders coupling scheme.

\begin{tabular}{|c|}
\hline $\mathrm{Fe}^{2+}$ terms \\
\hline${ }^{\mathbf{1}} \mathbf{S},{ }^{1} \mathbf{D},{ }^{1} \mathbf{G}$ \\
${ }^{3} \mathbf{P},{ }^{3} \mathbf{F}$ \\
${ }^{1} \mathbf{S},{ }^{1} \mathbf{D},{ }^{1} \mathbf{F},{ }^{1} \mathbf{G},{ }^{1} \mathbf{I}$ \\
${ }^{3} \mathbf{P},{ }^{3} \mathbf{D},{ }^{3} \mathbf{F},{ }^{3} \mathbf{G},{ }^{3} \mathbf{H}$ \\
${ }^{5} \mathbf{D}$
\end{tabular}

of the other terms are discussed in [27]. The only term of interest for 3-5 $\mu \mathrm{m}$ transitions is the ${ }^{5} \mathrm{D}$ term because, as we will see, this term is split by the $\mathrm{ZnSe}$ crystal field into states that are separated by about $2800 \mathrm{~cm}^{-1}$ [22]. The rest of the terms have splittings that lie above the ${ }^{5} \mathrm{D}$ term at energies much larger than $2800 \mathrm{~cm}^{-1}[22,29]$.

Group theory can be used to determine the effect a tetrahedral crystal field has on a D state such as the ${ }^{5} \mathrm{D}$ for $\mathrm{Fe}^{2+}$. A tetrahedral field has $\mathrm{T}_{d}$ symmetry in the cubic point group [27]. By calculating the characters of the matrices that express the effect of the $T_{d}$ symmetry operations on a $D$ state $(L=2)$, we can determine the irreducible representations 
that span a D state and thus the states that ${ }^{5} \mathrm{D}$ splits in to. The details of this calculation can be found in many books and so we will only quote the final result $[26-28,30]$. A tetrahedral crystal field splits a free-ion $D$ state into the $E$ and $T_{2}$ irreducible representations or states. The $E$ state is doubly degenerate and the $T_{2}$ state is triply degenerate [27]. For the case of $\mathrm{Fe}^{2+}$, the free-ion ${ }^{5} \mathrm{D}$ state has $\mathrm{L}=2$ and $\mathrm{S}=2$. However, for the resulting split $E$ and $T_{2}$ states, the angular momentum (L) is no longer a good quantum number because the tetrahedral crystal field partially lifts the degeneracy in the $m_{L}$ states of ${ }^{5} \mathrm{D}$ [31]. The crystal field does not affect the spin and therefore spin is still considered to be a good quantum number and since both split states will have $S=2$, the split states are labeled as ${ }^{5} \mathrm{E}$ and ${ }^{5} \mathrm{~T}_{2}$.

The splittings for all the $\mathrm{Fe}^{2+}$ free-ion states in a tetrahedral crystal field environment are shown on the Tanabe-Sugano diagram in Fig. 6.1 [28]. The relative values for the energies of the split states are specified in terms of $E / B$ as a function of $\Delta / B$. The crystal field strength parameter $(\Delta=10 \mathrm{Dq})$ specifies the size of the energy perturbation due to the crystal field [31] and here $\mathrm{E}$ is the absolute energy of the states measured in $\mathrm{cm}^{-1}$. In the case of $\mathrm{Fe}^{2+}: \mathrm{ZnSe}, \mathrm{Dq}$ can be expressed for a tetrahedral arrangement of $\mathrm{Se}^{2-}$ point charges surrounding the $\mathrm{Fe}^{2+}$ ion by

$$
D q \equiv \frac{280 e^{2}}{105 a^{2}} \int_{0}^{\infty} R_{3 d}^{2}(r) r^{4} r^{2} d r
$$

where $R_{3 d}^{2}(r)$ is the radial part of the $\mathrm{Fe}^{2+} 3 d$ orbitals, $a$ is the distance between the $\mathrm{Fe}^{2+}$ and $\mathrm{Se}^{2-}$ ions in the crystal, $\mathrm{r}$ is the distance from the center of the $\mathrm{Fe}^{2+}$. ion, and $\mathrm{e}$ is the charge of the electron. The $R_{3 d}^{2}(r)$ functions are not the hydrogenic $3 d$ atomic orbitals 
due to the interaction of the $\mathrm{Fe}^{2+}$ ion with the $\mathrm{ZnSe}$ crystal and the exact form of the $R_{3 d}^{2}(r)$ functions is not known. Therefore, the value for $D q$ is usually determined experimentally from spectroscopic measurements.

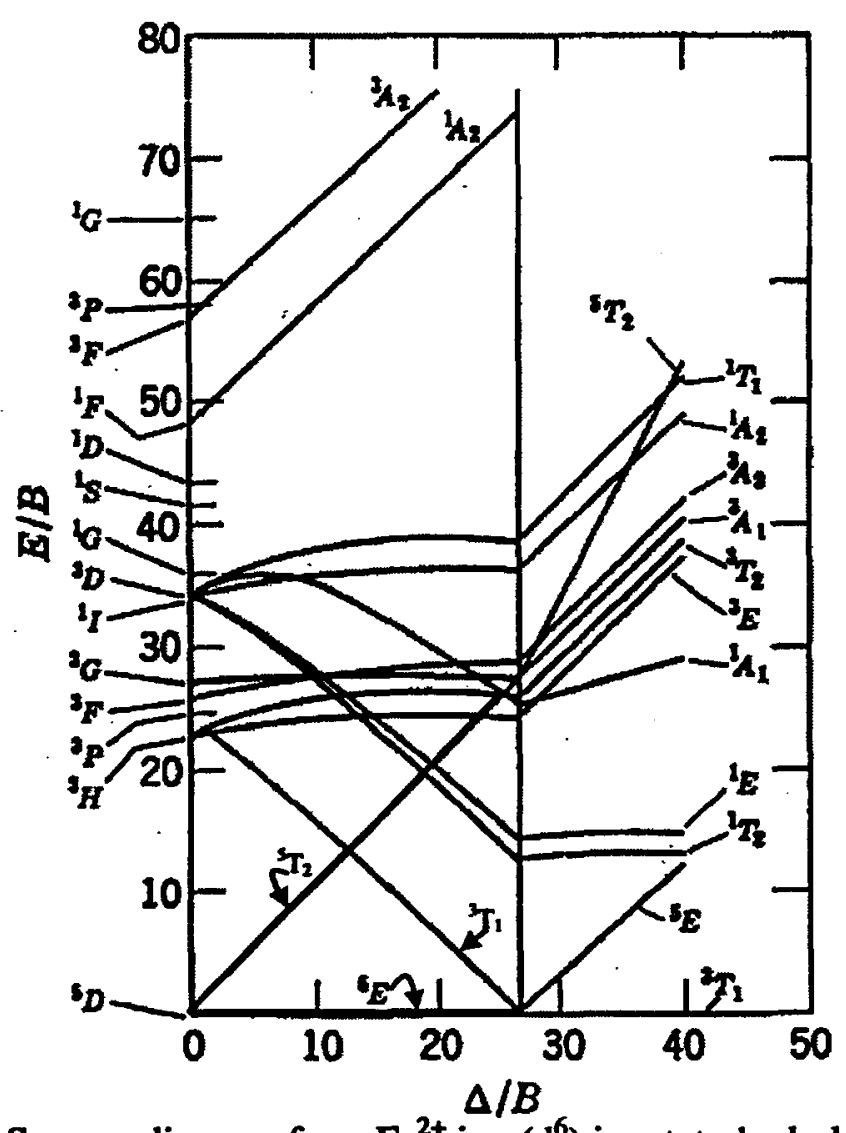

Figure 6.1. Tanabe-Sugano diagram for a $\mathrm{Fe}^{2+}$ ion $\left(\mathrm{d}^{6}\right)$ in a tetrahedral field [28].

The interaction energies of the electrons in the free-ion involve ten integrals over the wavefunctions of the electrons [28]. For d-electron systems, the interaction energies between the electrons can be reduced to three combinations of the ten integrals and these three combinations are known as the Racah A, B, and C parameters [28]. Linear combinations of the Racah B and C parameters are equal to the energy differences between the free-ion states (far left of Fig. 6.1). The Racah B and C parameters refer to the energy differences that occur between states that have different spatial symmetries 
and therefore have different values for the non-spherical repulsion energy of the electrons in the partially filled orbitals [31]. If the ratio C/B is known, then the energy differences between the free-ion terms can be expressed solely in terms of $B$. The individual values for the $\mathrm{B}$ and $\mathrm{C}$ parameters can be readily determined from experiments conducted, for instance, with gaseous free-ions. The ratio of $\mathrm{C} / \mathrm{B}$ for the free-ions of the first transition series have been found to have values that lie between 4 and 5 with only small variation $(<10 \%)$ between the different ions [28].

The vertical separations in energy of the states on the left side (i.e. $\Delta=0$ ) of the Tanabe-Sugano diagram in Fig. 6.1 are determined from the values of $B$ and $C$. In the derivation of the Tanabe-Sugano diagrams, a free-ion value for $C / B=5$ is assumed which eliminates $\mathrm{C}$ as a parameter in the calculation [28]. The free-ion value for $\mathrm{C} / \mathrm{B}$ is a good approximation for transition metal ions that are in crystals because the orbitals in the crystals do not deviate by a large amount from the free-ion orbitals [28]. A value for C/B $=4.61$ was used in the calculation of the diagram in Fig. 6.1. A further simplification in the calculation of the Tanabe-Sugano diagram is accomplished by dividing the energy " matrix (Hamiltonian) by the Racah parameter B [28]. The calculated energy eigenvalues are then in units of $B$ (i.e. $E / B$ ). Note that the energy then becomes a function of $\Delta / B$ and so the energy of the terms $(E / B)$ are plotted vs. $\Delta / B$ on the Tanabe-Sugano diagrams.

It is expected from results for $\mathrm{Cr}^{2+}: \mathrm{ZnSe}[24]$ and the $\mathrm{Fe}^{2+}$ free-ion value for $\mathrm{B}(917$ $\mathrm{cm}^{-1}$ ) that $\Delta / \mathrm{B}<10$ for $\mathrm{Fe}^{2+}: \mathrm{ZnSe}$ which implies that its ground state will be the ${ }^{5} \mathrm{E}$ state and the first excited state will be ${ }^{5} \mathrm{~T}_{2}$ according to Fig. 6.1. One of the goals of this spectroscopic study is to determine where $\mathrm{Fe}^{2+}: \mathrm{ZnSe}$ is in the range $0<\Delta \mathrm{B}<27$ on the Tanabe-Sugano diagram. We expect the energy separation between the ${ }^{5} \mathrm{E}$ and ${ }^{5} \mathrm{~T}_{2}$ states 
in $\mathrm{Fe}^{2+}: \mathrm{ZnSe}$ to be $\sim 2800 \mathrm{~cm}^{-1}$ and since this is the only transition in the wavelength range we desire $(\sim 4 \mu \mathrm{m})$, we will now focus solely on the fine structure of the ${ }^{5} E$ and ${ }^{5} T_{2}$ states.

The work by Slack et al. [20] suggests that accounting for spin-orbit interactions to $2^{\text {nd }}$ order is sufficient enough to describe the observed spectra of $\mathrm{Fe}^{2+}$ ions in crystals like $\mathrm{ZnSe}$. The Hamiltonian given in Eqn. (6.1) has two terms that give rise to $1^{\text {st }}$ and $2^{\text {nd }}$ order spin-orbit splittings. Group theory can be used to calculate the representations or terms that the ${ }^{5} \mathrm{E}$ and ${ }^{5} \mathrm{~T}_{2}$ states are split into by the spin-orbit coupling $[20,27]$. The first thing that is needed is the $T_{d}$ symmetry group representation for $S=2$. Angular momentum that is equal to 2 can be represented by the combination $\left(E+T_{2}\right)$ [32]. The spin-orbit operator in the Hamiltonian has the form of LeS. Given this form (multiplication) for the spin-orbit operator, we can determine the terms that the ${ }^{5} E$ and ${ }^{5} T_{2}$ states split into by multiplying the characters of the $S=2$ spin representation $\left(E+T_{2}\right)$ by the characters of either the ${ }^{5} \mathrm{E}$ (orbital doublet) or ${ }^{5} \mathrm{~T}_{2}$ (orbital triplet) states. The characters of these representations in the $T_{d}$ symmetry group can be found in standard group theory character tables [27]. The terms that ${ }^{5} \mathrm{E}$ and ${ }^{5} \mathrm{~T}_{2}$ split into are the terms in the $T_{d}$ group whose linear combination have characters that are equal to the characters of either $\left(E+T_{2}\right) \times{ }^{5} E$ or $\left(E+T_{2}\right) \times{ }^{5} T_{2}$. Performing this procedure we obtain:

$$
\begin{gathered}
\left(E+T_{2}\right) \times{ }^{5} E=A_{1}+A_{2}+E+T_{1}+T_{2} \\
\left(E+T_{2}\right) \times{ }^{5} T_{2}=A_{1}+E+2 T_{1}+2 T_{2}
\end{gathered}
$$

The eleven states on the right are the total number of spin-orbit states that the ${ }^{5} \mathrm{E}$ and ${ }^{5} \mathrm{~T}_{2}$ states could be split into by the spin-orbit coupling. One thing to note about these spin- 
orbit states is that they have lost their spin designation because spin is no longer a good quantum number due to the spin-orbit coupling.

Calculations by Low and Weger [32] and Baranowski et al. [29] find that the spinorbit states of the ${ }^{5} \mathrm{E}$ term are not split in the $1^{\text {st }}$ order spin-orbit coupling but split into equally spaced levels in the $2^{\text {nd }}$ order coupling. The spin-orbit states of the ${ }^{5} \mathrm{~T}_{2}$ term split in both $1^{\text {st }}$ and $2^{\text {nd }}$ order and have various spacings [32]. Adopting this general scheme for the spacings of the split states, a partial energy level diagram for $\mathrm{Fe}^{2+}$ in a tetrahedral field showing the crystal field splitting and the first and second-order spin-orbit splitting of the ${ }^{5} \mathrm{D}$ free-ion state is shown in Fig. 6.2. The spacings for the ${ }^{5} \mathrm{~T}_{2}$ spin-orbit states should not be regarded as exact but are schematically shown based on general trends found in the literature $[20,21,32]$.

Baranowski et al. [29] have derived expressions that can be used to calculate the energy spacing of the 11 spin-orbit states shown in Fig. 6.2. The expressions are in terms of the crystal field strength parameter $(\Delta)$ and the spin-orbit interaction parameter $(\lambda)$. Choosing level 6 to be at "zero" energy, Eqns. (6.5) - (6.14) give the energy spacing between level 6 and the other 10 spin-orbit states. Their calculation only accounts for crystal field and spin-orbit effects and neglects effects like the static Jahn-Teller effect [20], the Ham effect [29], etc. The notation $E_{(i-6)}$ specifies the energy difference between experimentally measured absorption and emission spectra.

We are interested in the optical transitions between the spin-orbit states in Fig. 6.2 so the transition selection rules between these states are needed. Group theory can be used to determine the selection rules for electric dipole transitions between the spin-orbit states in Fig. 6.2 [27]. We will only consider electric dipole transitions. The electric dipole 


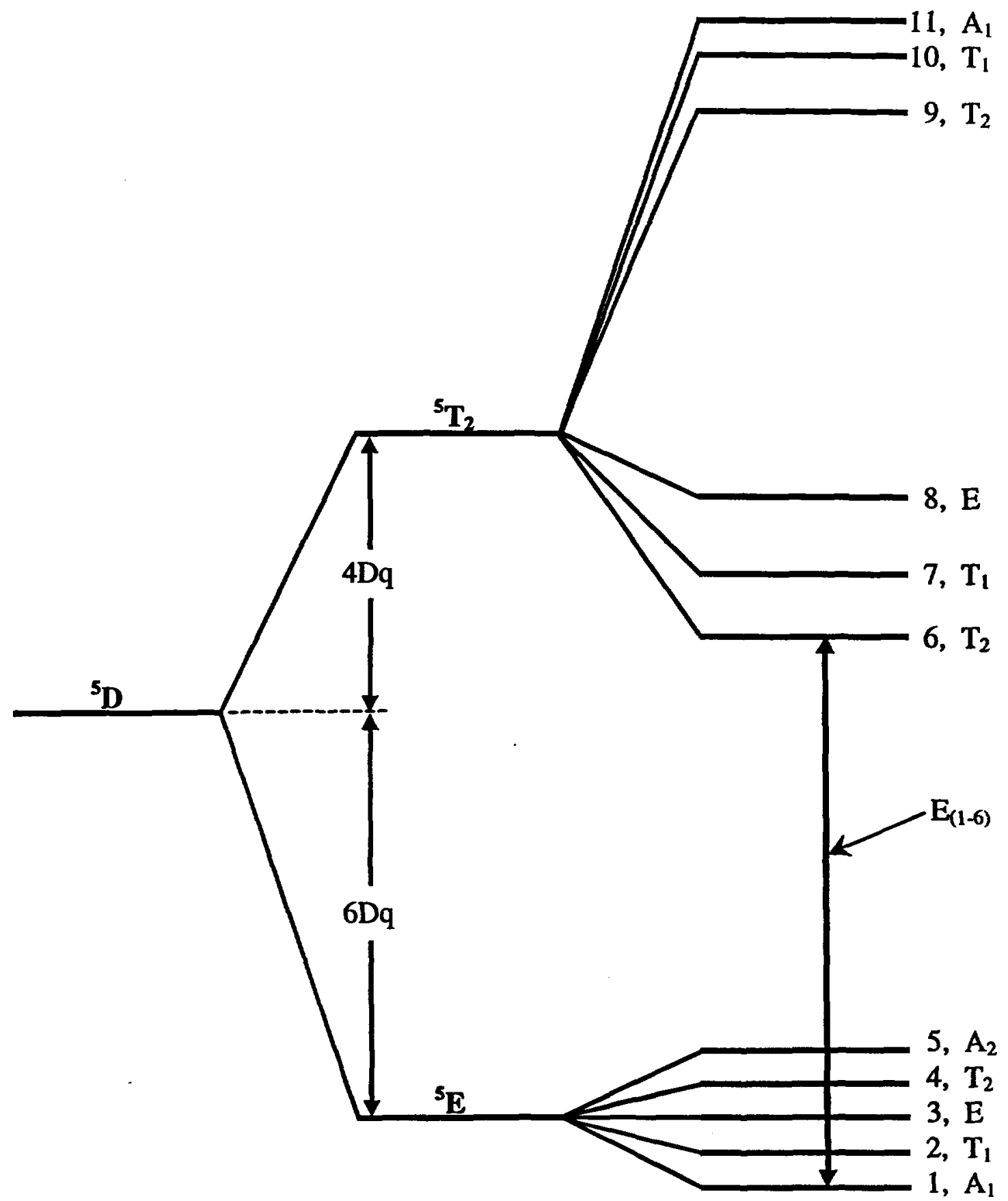

Free-ion

state
Tetrahedral

Crystal Field splitting
First and Second-Order Spin-Orbit splitting

Figure 6.2. Splittings of the ${ }^{5} \mathrm{D}$ free-ion state due to a tetrahedral crystal field and spinorbit coupling. The spin-orbit levels are labeled 1 through 11 for convenience and their group theory symmetry state is also given. The energy difference $\mathrm{E}_{(1-6)}$ is not to scale. The labeling of $4 \mathrm{Dq}$ and $6 \mathrm{Dq}$ is standard for cubic crystal fields [27]. 
operator in the $T_{d}$ symmetry group has $T_{2}$ symmetry. This is because the dipole operator transforms like a spatial coordinate $(x, y, z)$ and the spatial coordinates in the $T_{d}$ symmetry are represented by $T_{2}$. This can be found in standard group theory character tables [27].

$$
\begin{aligned}
& \mathrm{E}_{(1-6)}=\Delta+3 \lambda+(138 / 5) \lambda^{2} / \Delta \\
& \mathrm{E}_{(2-6)}=\Delta+3 \lambda+(108 / 5) \lambda^{2} / \Delta \\
& \mathrm{E}_{(3-6)}=\Delta+3 \lambda+(78 / 5) \lambda^{2} / \Delta \\
& \mathrm{E}_{(4-6)}=\Delta+3 \lambda+(48 / 5) \lambda^{2} / \Delta \\
& \mathrm{E}_{(5-6)}=\Delta+3 \lambda+(18 / 5) \lambda^{2} / \Delta \\
& \mathrm{E}_{(7-6)}=-2 \lambda+(12 / 5) \lambda^{2} / \Delta \\
& \mathrm{E}_{(8-6)}=-2 \lambda+(42 / 5) \lambda^{2} / \Delta \\
& \mathrm{E}_{(9-6)}=-5 \lambda-(6 / 5) \lambda^{2} / \Delta \\
& \mathrm{E}_{(10-6)}=-5 \lambda+(42 / 5) \lambda^{2} / \Delta \\
& \mathrm{E}_{(11-6)}=-5 \lambda+(102 / 5) \lambda^{2} / \Delta
\end{aligned}
$$

A transition between two states is allowed when the result of the multiplication of the characters of the two states contains the character of the dipole operator, $T_{2}$. This then implies that the subsequent multiplication by the dipole operator will make the integrand contain the totally symmetric representation $A_{1}$ and the result of the integration can be non-zero. The integrand must contain $A_{1}$ symmetry to be non-zero since $A_{1}$ possesses the full symmetry of the group. Table 6.2 gives the electric dipole selection rules between the states shown in Fig. 6.2.

Up to this point we have treated the crystal field as due to a static or motionless arrangement of charges. In reality for a system like $\mathrm{Fe}^{2+}: \mathrm{ZnSe}$, the $\mathrm{ZnSe}$ lattice is 
Table 6.2. Selection rules for electric-dipole transitions between the spinorbit levels in Fig. 6.2.

\begin{tabular}{|c|c|c|c|c|}
\hline Initial States $\left({ }^{5} \mathrm{E}\right)$ & \multicolumn{5}{|c|}{ Final States $\left({ }^{5} \mathrm{~T}_{2}\right)$} \\
\hline & $11\left(\mathrm{~A}_{1}\right)$ & $8(\mathrm{E})$ & $7,10\left(\mathrm{~T}_{1}\right)$ & $6,9\left(\mathrm{~T}_{2}\right)$ \\
\hline $1\left(\mathrm{~A}_{1}\right)$ & F & F & F & A \\
\hline $2\left(\mathrm{~T}_{1}\right)$ & F & A & A & A \\
\hline $3(\mathrm{E})$ & F & F & A & A \\
\hline $4\left(\mathrm{~T}_{2}\right)$ & A & A & A & A \\
\hline $5\left(\mathrm{~A}_{2}\right)$ & F & F & A & F \\
\hline
\end{tabular}

$\mathrm{F}=$ forbidden and $\mathrm{A}=$ allowed.

vibrating. Therefore the $\mathrm{Fe}^{2+}$ ions "see" a vibrating crystal field. Since the optical transitions that we are interested in occur in the d orbitals and these are the same orbitals that bond $\mathrm{Fe}^{2+}$ to the $\mathrm{ZnSe}$ lattice, we can expect the vibration of the lattice to produce noticeable effects in the spectra [30]: Henderson and Imbush [30] discuss how the vibration of the host crystal can cause broadening in the absorption and emission spectra and shorten the luminescence lifetime and that these effects depend on the temperature of the crystal. The temperature dependent effects in the spectra depend on the strength of the vibrational coupling. Typically [33], at low temperatures, say $10 \mathrm{~K}$, the spectra are narrower and have a Pekarian form and at higher temperatures, say $200 \mathrm{~K}$, the spectra are broader and have a Gaussian form [30]. The effects are usually much more apparent for transition metal ions than for rare-earth metal ions since the optical transitions in rareearth ions typically occur in orbitals (f-shell) that are shielded from the crystal field by the bonding orbitals. With the foresight that we will encounter these effects in the spectra of $\mathrm{Fe}^{2+}: \mathrm{ZnSe}$, we would like to now develop a simple model that we can use to quantify these effects [34]. 
The vibrational modes of $\mathrm{Fe}^{2+}: \mathrm{ZnSe}$ will be treated as harmonic or in other words described by harmonic oscillators. However, the bonds between the $\mathrm{Fe}^{2+}$ ions and the $\mathrm{ZnSe}$ lattice will not be treated like they have potential energy that is proportional to $\mathrm{x}^{2}$. A simpler but still useful model is to assume that the potential energy of the coupling between the $\mathrm{Fe}^{2+}$ ions and the $\mathrm{ZnSe}$ lattice is linear in the displacement between the $\mathrm{Fe}^{2+}$ and $\mathrm{Se}^{2-}$ ions, i.e. proportional to $\mathrm{x}$. This assumption is the basis for the "linear-coupling model" [35]. (The assumption is equivalent to using only the first term in a Taylor series expansion for the coupling potential energy.) Results from experiments on other materials indicate that this model is in general a reasonably good description of the physical situation $[33,34,36]$. The major result of the linear coupling model is that the excited states of the system have an increased equilibrium distance between the ions but have the same energy as if there were no vibrational coupling.

\subsubsection{Configurational coordinate diagram}

A simple pictorial description of the linear coupling model is a configurational coordinate diagram. A basic configurational coordinate diagram is shown in Fig. 6.3. On the diagram two curves are drawn as a function of the configurational coordinate $Q$, one that represents the adiabatic potential energy surface for the ground state and one for the excited state. The configurational coordinate $(Q)$ represents the equilibrium position of the ions in the lattice. Note that the ground state and the excited state have different values for $Q, Q(g)$ and $Q(e)$ respectively. The horizontal lines in each of the curves represent the equally spaced vibrational levels of the system. The vibrational levels are equally spaced because we are assuming that the ground state and the excited state have the same vibrational frequency $\omega_{b}$ (i.e. the Rhys approximation of only a single 
vibrational mode [30]). $\mathrm{E}_{\mathrm{ZPL}}$ is the transition energy of the "zero-phonon line". A zero phonon line corresponds to a transition that occurs without the emission or absorption of lattice vibrational energy (phonons). Optical transition are represented by vertical lines on the configuration coordinate diagram because, in accordance with the Franck-Condon principle [30], electronic transitions (i.e. optical) occur much faster than the change in positions of the nuclei.

The intersection of the ground and excited state on the configurational coordinate diagram provides a path for nonradiative relaxation from the electronic excited state to the ground state. The nonradiative processes that couple the excited state to the ground state are thermally-activated (i.e. a function of temperature). The energy of the crossing point $\left(\mathrm{E}_{\mathrm{A}}\right.$ as measured on the diagram) is the lattice thermal activation energy required to change from the excited state configuration to the ground state configuration [35]. Another way to look at this is that $\mathrm{E}_{\mathrm{A}}$ is the energy barrier that the lattice must overcome in order to change from the excited state configuration to the ground state configuration [35]. The thermal activation energy $\left(E_{A}\right)$ is related to the thermally induced nonradiative transition rate $\left(\mathrm{W}_{\mathrm{nr}}\right)$ by $[34]$

$$
W_{n r}=W_{n r}^{\circ} e^{\frac{-E_{A}}{k T}}
$$

where $\mathrm{W}_{\mathrm{nr}}^{0}$ is the high temperature thermal nonradiative transition rate, $\mathrm{k}$ is Boltzmann's constant, and $\mathrm{T}$ is the temperature in Kelvin. Eqn. (6.15) has units of $\mathrm{s}^{-1}$. The total transition rate out of the excited state, which is sum of the radiative and the nonradiative 


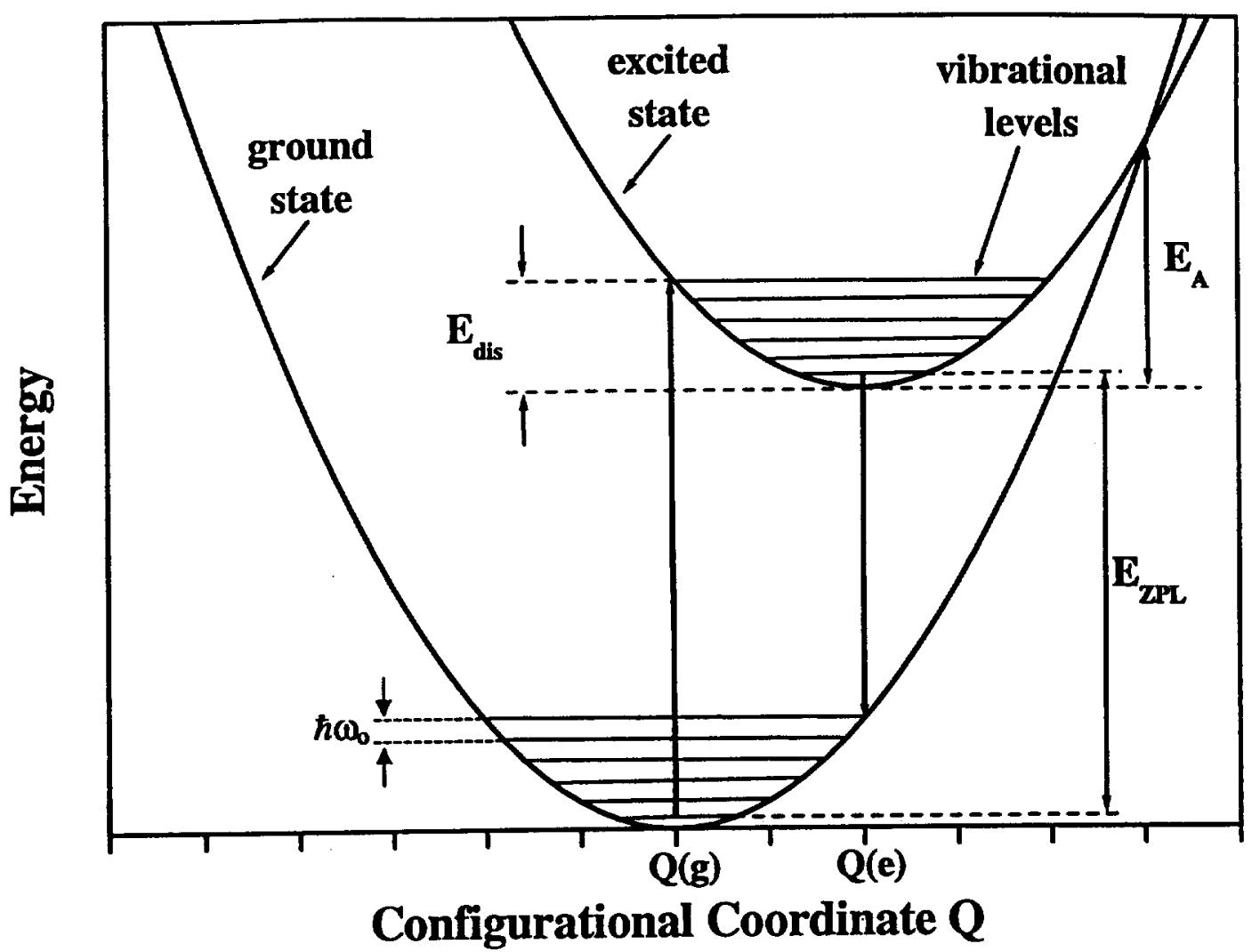

Figure 6.3. Basic configurational coordinate diagram based on the linear coupling model [30].

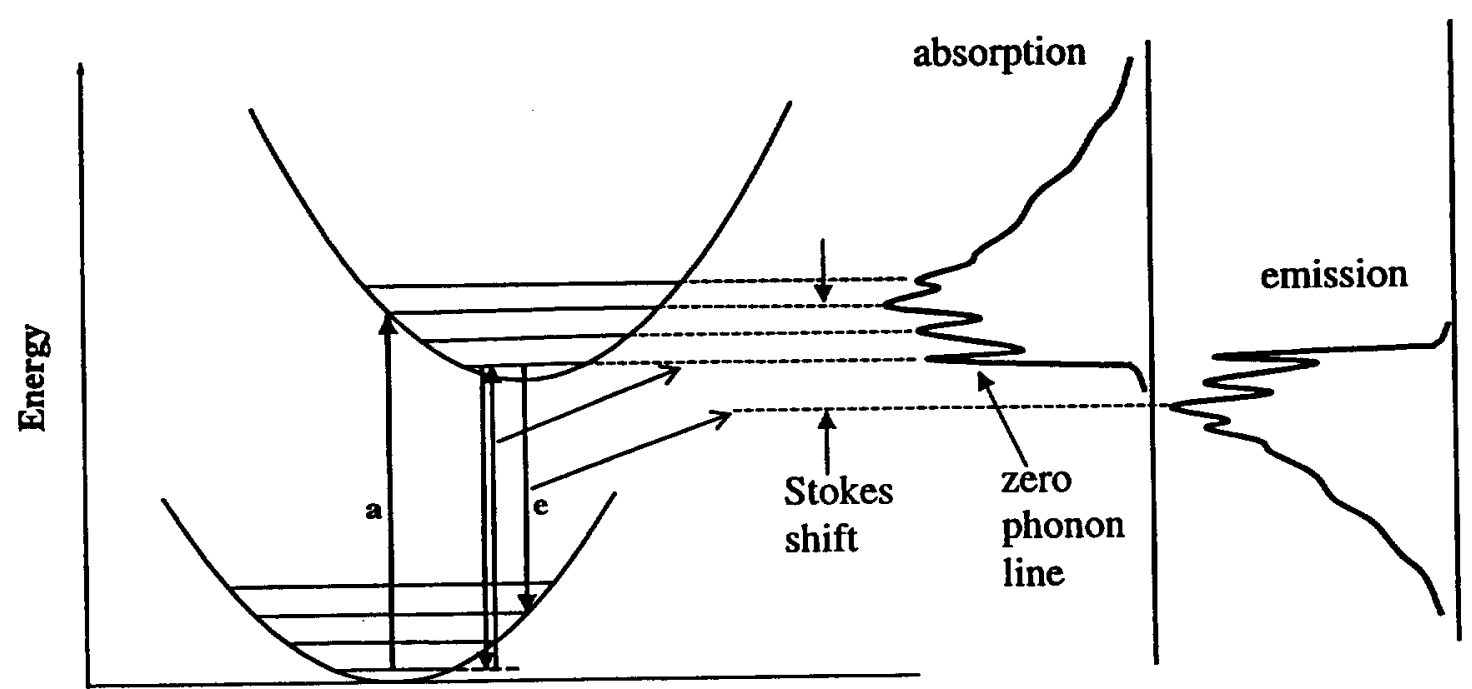

Configurational Coordinate

Figure 6.4. Relation between the configurational coordinate diagram and the low temperature spectra showing the zero phonon line and the Stokes shift [30]. 
rates give the luminescence lifetime. The total transition rate [36] $\left(W_{\text {total }}\right)$ can be written as

$$
\mathrm{W}_{\text {total }}=\mathrm{W}_{\text {radiative }}+\mathrm{W}_{\mathrm{nr}}
$$

where $\mathrm{W}_{\text {radiative }}$ is the transition rate for emission of photons and $\mathrm{W}_{\mathrm{nr}}$ is the nonradiative rate. For $\mathrm{Fe}^{2+}: \mathrm{ZnSe}$, we will assume that $\mathrm{W}_{\mathrm{nr}}$ is given by Eqn. (6.15) since the nonradiative processes in this system are thermally-activated (phonons) $[20,21]$. The luminescence lifetime is equal to the inverse of $W_{\text {total }}$ as given by

$$
\tau_{\text {luminessence }}=\frac{1}{W_{\text {total }}}=\frac{1}{W_{\text {radiative }}+W_{n r}^{\circ} e^{-\frac{E_{A}}{k T}}}
$$

The luminescence lifetime is the lifetime that will be measured by experiments.

$E_{\text {dia }}$ in Fig. 6.3 is the vibrational energy that is released after a transition occurs along the vertical line at $Q(g)$ and the system relaxes to equilibrium [37]. $E_{\text {dis }}$ can be related to the characteristic phonon frequency, $\omega_{b}$, by

$$
\mathrm{E}_{\mathrm{dis}}=(\mathrm{m}+1 / 2) \hbar \omega_{0}
$$

where $m$ is an integer that designates the vibrational level. It is standard to use Eqn. (6.18) to define the Huang-Rhys parameter (S) [30] as

$$
S \equiv \frac{E_{d i s}}{\hbar \omega_{0}}
$$

Therefore, $\mathrm{S}$ is the number of phonons emitted after an absorption transition takes place along the vertical line at $Q(\mathrm{~g})$. Values for $S<6$ are referred to as the weak coupling 
regime and values $>6$ as the strong coupling regime. These regimes for $S$ are based on low temperature spectra where for values of $S<6$ the zero phonon line can be seen along with the phonon sideband and for values of $S>6$ the zero phonon line cannot be seen and the phonon sideband totally dominates the spectra [30]. An average value for $S$ will be determined for $\mathrm{Fe}^{2+}: \mathrm{ZnSe}$ from the data collected in this study.

Two very useful relations can be derived among the various parameters that are labeled in Fig. 6.3. By treating $Q(g)$ and $Q(e)$ as the displacements of harmonic oscillators that represent the ground and excited states, respectively, an expression can be derived that relates the Huang-Rhys parameter to $Q(g)$ and $Q(e)$ as

$$
S=\frac{1}{2} \frac{M \omega_{0}}{\hbar}(\mathrm{Q}(\mathrm{e})-\mathrm{Q}(\mathrm{g}))^{2}
$$

where $M$ is an effective ionic mass and $\omega_{0}$ is the phonon frequency of the system. We are implicitly assuming in the linear coupling model that there is a single vibrational mode, which is the fully symmetric "breathing mode" [35]. In the breathing mode, the $\mathrm{Fe}^{2+}$ ion is stationary and the $\mathrm{Se}^{2-}$ ions vibrate radially in-phase. If we choose the distance between the $\mathrm{Fe}^{2+}$ ion and its $\mathrm{Se}^{2-}$ nearest neighbors to be $\mathrm{Q} / 2$, then the mass $(\mathrm{M})$ in Eqn. (6.20) is equal to the mass of $\mathrm{Se}^{2-}$. The term $(\mathrm{Q}(\mathrm{e})-\mathrm{Q}(\mathrm{g}))$ is referred to as the Condon offset and describes how much the excited state is displaced horizontally from the ground state on the configuration coordinate diagram. An expression for $\mathrm{E}_{\mathrm{A}}$ can also be derived by treating $Q(\mathrm{~g})$ and $\mathrm{Q}(\mathrm{e})$ as the displacements of harmonic oscillators and using the definition of $S$ in terms of $E_{\text {dis. }} E_{A}$ is then given by 


$$
E_{A}=\frac{\left(E_{Z P L}-S \hbar \omega_{0}\right)^{2}}{4 S \hbar \omega_{0}}
$$

Both Eqn. (6.20) and (6.21) will be used in the analysis of the experimental data.

The horizontal displacement of the curves in Fig. 6.3, (i.e. the different values of $Q$ ) is a direct result of the linear nature of the coupling between the $\mathrm{Fe}^{2+}$ ions and the vibrations of the host lattice. (When a potential that is linear in displacement is added to a harmonic oscillator potential, the effect on the harmonic oscillator is that its excited state energy remains the same but the equilibrium displacement of the state is changed, i.e. Fig. 6.3) The displacement in Fig. 6.3 produces sidebands in the spectra as shown in Fig. 6.4. Another way to see how these sidebands arise is to realize that the vibration of the lattice about the $\mathrm{Fe}^{2+}$ ion modulates the optical transition by $\omega_{0}$, which in Fourier theory amounts to putting frequency sidebands in the tranisition's frequency spectrum. Phonon sidebands appear in both the absorption and emission spectra. The peak in the phonon sideband in the absorption spectrum occurs at an energy that corresponds to the length of the upward arrow labeled "a" in Fig. 6.4. The peak in the phonon sideband in the emission spectrum occurs at an energy that corresponds to the length of the downward arrow labeled "e" in Fig. 6.4 hence the phonon sideband peak is at a lower energy than the zero phonon line. The difference in energy between the peak in the phonon sideband in the absorption spectrum and the peak in the phonon sideband in the emission spectrum is defined as the Stokes shift as shown in Fig. 6.4. If the ground and excited states have equal phonon energies, as we are assuming in the Rhys approximation, the absorption and emission spectra will have sideband peaks that are equidistant in energy from the zero phonon line. In this case, the Stokes shift [26] is 
related to the Huang-Rhys factor $(S)$ and the lattice phonon energy $\left(\hbar \omega_{0}\right)$ by,

$$
\text { Stokes shift }\left(\mathrm{cm}^{-1}\right)=(2 \mathrm{~S}-1) \hbar \omega_{b}
$$

\subsection{Spectroscopy experiments and results}

\subsection{Sample preparation}

Samples of $\mathrm{Fe}^{2+}$-doped $\mathrm{ZnSe}$ were produced by two methods. A boule of $\mathrm{Fe}^{2+}: \mathrm{ZnSe}$ was grown by Eagle-Picher Research Laboratory, Inc. using the modified vertical Bridgman growth method. The melt was about 0.06 at.\% rich in FeSe. The modified vertical Bridgman growth method [38] entails taking a sealed graphite ampoule containing powdered $\mathrm{ZnSe}$ and $\mathrm{FeSe}$ in the desired proportions and slowly passing this ampoule vertically through a hot melting zone and then a cooling zone where the contents in the ampoule crystallize into $\mathrm{Fe}^{2+}: \mathrm{ZnSe}$. This method has the advantage that large crystals can easily be obtained because of the relatively fast growth rate. The boule produced by Eagle-Picher Research Laboratory, Inc. was approximately $12 \mathrm{~mm}$ long by $20 \mathrm{~mm}$ in diameter and possessed an $\sim 100$ percent concentration gradient (determined at LLNL using inductively coupled plasma and atomic-emission spectrometry (ICP-AES)) of $\mathrm{Fe}$ along the vertical direction. The boule was polycrystalline with single crystal domains as large as $3-4 \mathrm{~mm}^{3}$. Samples for spectroscopic analysis were cut mainly from the boules' single crystal regions in slabs approximately $2 \times 10 \times 10 \mathrm{~mm}^{3}$.

$\mathrm{Fe}^{2+}$-doped $\mathrm{ZnSe}$ samples were also obtained using a diffusion doping method [39]. This process involves placing an $\sim 5 \times 10 \times 10 \mathrm{~mm}^{3}$ polished slab of $\mathrm{ZnSe}$ into a quartz test tube along with FeSe powder, evacuating and sealing the tube, and then heating the tube 
and contents in a furnace at $1000^{\circ} \mathrm{C}$ for approximately 5 days. $\mathrm{Fe}^{2+}$ ions diffuse into the $\mathrm{ZnSe}$ crystal material (with a diffusion coefficient of $\cong 1.5 \times 10^{-7} \mathrm{~cm}^{2} / \mathrm{s}$ ) and substitute into the $\mathrm{Zn}^{2+}$ sites within the $\mathrm{ZnSe}$ lattice. The slabs of pure $\mathrm{ZnSe}$ are easily obtained from single crystal infrared window material. The diffusion doping process produces uniformly doped samples with the level of doping dependent on the diffusion time and temperature [25,39]. The diffusion doping process is attractive because it can produce homogeneous doped samples relatively simply and quickly with inexpensive and easily obtained starting materials and standard laboratory equipment.

The concentration of $\mathrm{Fe}$ in several of the $\mathrm{Fe}^{2+}$-doped $\mathrm{ZnSe}$ samples was measured at LLNL using ICP-AES. This analysis determined the total concentration of atomic Fe in the samples with an uncertainty of $\pm 15 \%$ without regard to the oxidation state. Table 6.3 lists the samples analyzed and the Fe concentrations. Sample \#12163 was obtained from

Table 6.3. Fe concentration in the $\mathrm{Fe}^{2+}: \mathrm{ZnSe}$ samples as determined by ICP-AES.

\begin{tabular}{|c|c|c|}
\hline Sample & Method of production & $\begin{array}{c}\text { Fe concentration } \\
\left(10^{19} \mathrm{Fe} \text { atoms } / \mathrm{cm}^{3}\right)\end{array}$ \\
\hline $\mathrm{Fe}^{2+}: \mathrm{ZnSe} \# 12163$ & Bridgman & 3.40 \\
\hline $\mathrm{Fe}^{2+}: \mathrm{ZnSe} \# 740$ & Diffusion doping & 0.302 \\
\hline $\mathrm{Fe}^{2+}: Z n S e \# 12464$ & Diffusion doping & 0.0282 \\
\hline $\begin{array}{c}\text { Undoped ZnSe window } \\
\text { material }\end{array}$ & - & 0.0272 \\
\hline
\end{tabular}

the Bridgman grown boule. Samples \#740 and \#12464 were prepared by the diffusion doping method from $\mathrm{ZnSe}$ window material. The diffusion-doped samples have several orders of magnitude less $\mathrm{Fe}$ than the samples produced by the Bridgman method. Therefore, in order to be a viable alternative to Bridgman growth, the diffusion doping 
method needs to be optimized (i.e. undoped slab size, starting chemical composition, heating time, etc.) to produce doping levels comparable to the Bridgman growth.

\subsubsection{Temperature dependent absorption spectra}

Unpolarized absorption spectra were collected on the set of $\mathrm{Fe}^{2+}: \mathrm{ZnSe}$ samples in Table 6.3. The spectra were measured at different temperatures for wavenumbers from 2000-5000 $\mathrm{cm}^{-1}$ using a Perkin-Elmer model 983 spectrophotometer. The spectra were collected with the samples mounted in an Air Products Displex 202 closed cycle helium gas cryogenic system. The cryogenic system could vary the sample's temperature from approximately $14 \mathrm{~K}$ to $300 \mathrm{~K}$. The sample in the cryogenic mount was positioned into the spectrophotometer's sample compartment and then spectra were measured at various temperatures. The spectrophotometer measured the absorption of the sample in units of optical density (OD). The optical density values were then converted to absorption crosssection $\left(\sigma_{\mathrm{abs}}\right)$ using the relation that $\sigma_{\mathrm{abs}}(\lambda)=\frac{(\mathrm{OD}(\lambda))(2.303)}{\mathrm{N} \ell}$ where $\mathrm{N}$ is the number density of Fe from Table 6.3 and $\ell$ is the length of the sample. The measured absorption spectra for the $\mathrm{Fe}^{2+}: \mathrm{ZnSe}$ samples are presented in terms of absorption cross-section in Figs. 6.5 - 6.8. The sharp peaks in Fig. 6.5 between $2650 \mathrm{~cm}^{-1}$ and $2750 \mathrm{~cm}^{-1}$ are the zero-phonon transitions and the broad band extending $>2800 \mathrm{~cm}^{-1}$ is the phonon sideband. The values for the cross-sections for $\mathrm{Fe}^{2+}: \mathrm{ZnSe}$ are about an order of magnitude greater than that for Nd:YAG.

The spectra in Fig. 6.6 illustrate how the broadening due to the vibrational coupling of the $\mathrm{Fe}^{2+}$ ions with the $\mathrm{ZnSe}$ lattice increases with temperature. The broadening is due 


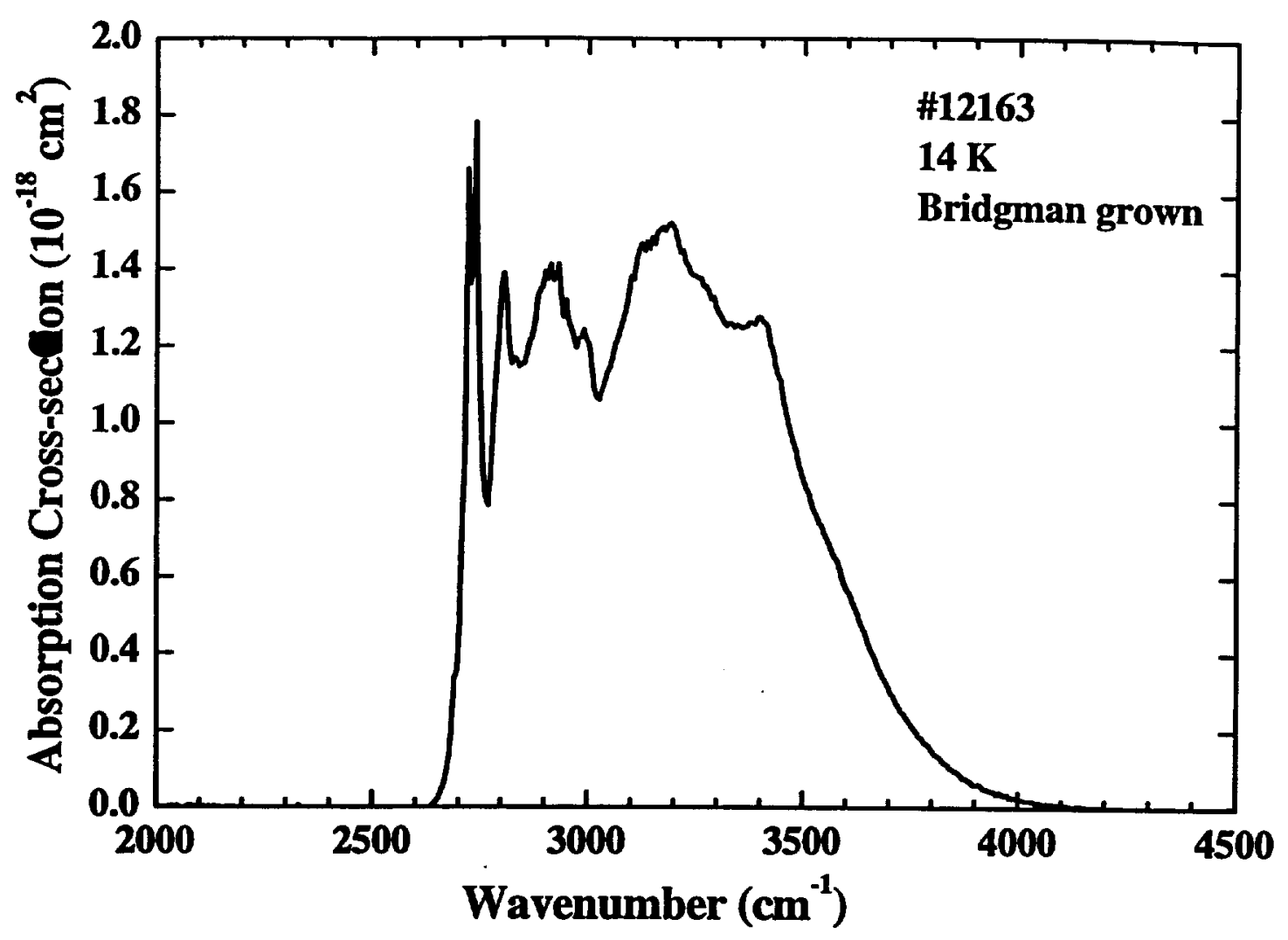

Figure 6.5. Unpolarized absorption spectrum at $14 \mathrm{~K}$ for $\mathrm{Fe}^{2+}: \mathrm{ZnSe}$ sample \#12163.

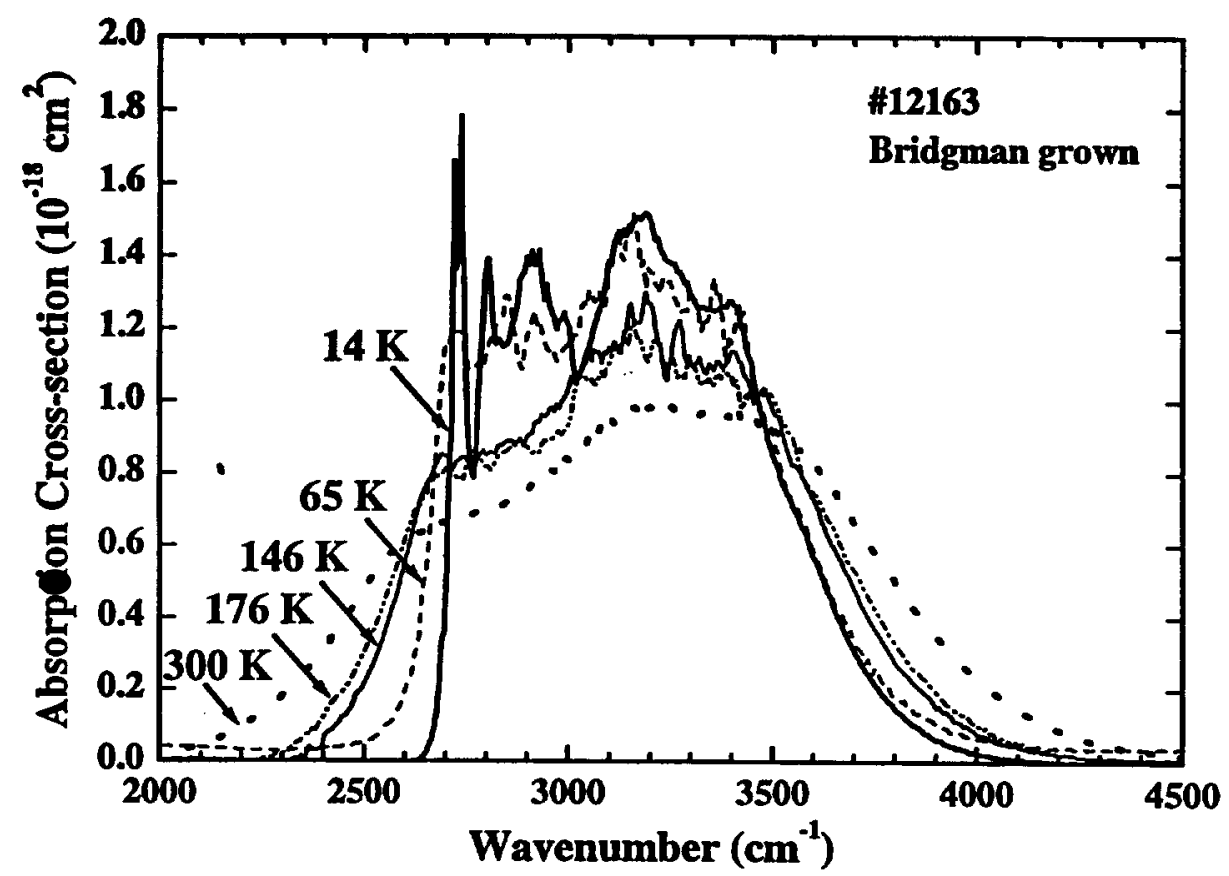

Figure 6.6. Unpolarized absorption cross-sections at temperatures between $14 \mathrm{~K}$ and 300 $\mathrm{K}$ for $\mathrm{Fe}^{2+}: \mathrm{ZnSe}$ sample \#12163. 
to the thermal increase in population in the upper spin-orbit and vibrational levels of the ${ }^{5} \mathbf{E}$ state.

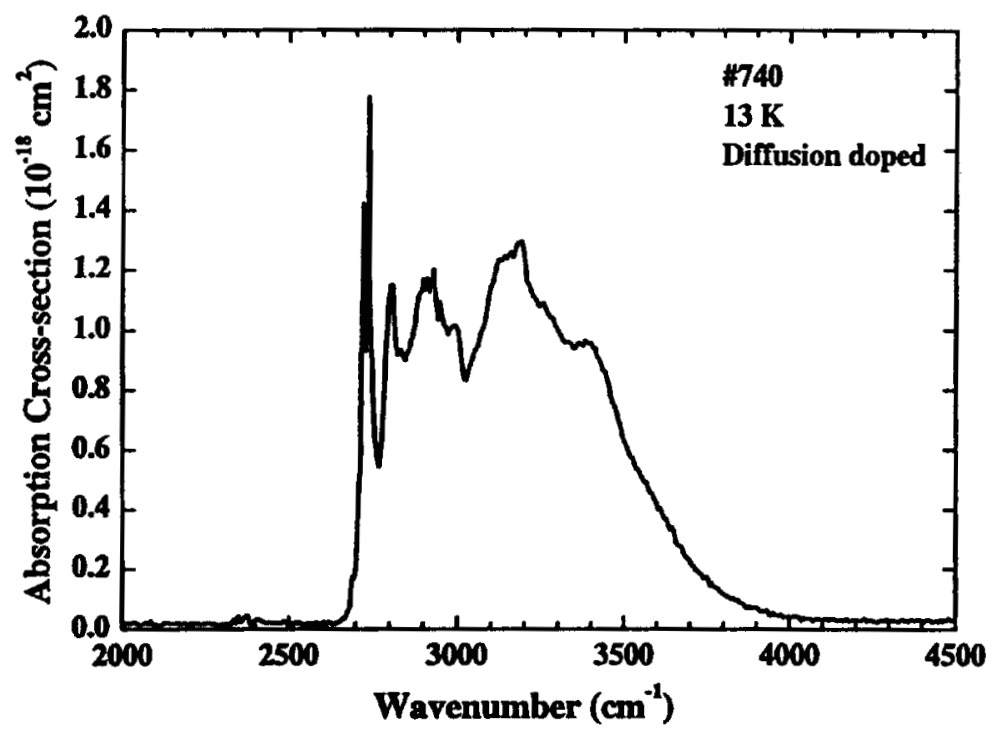

Figure 6.7. Unpolarized absorption spectrum at $13 \mathrm{~K}$ for $\mathrm{Fe}^{2+}: \mathrm{ZnSe}$ sample \#740.

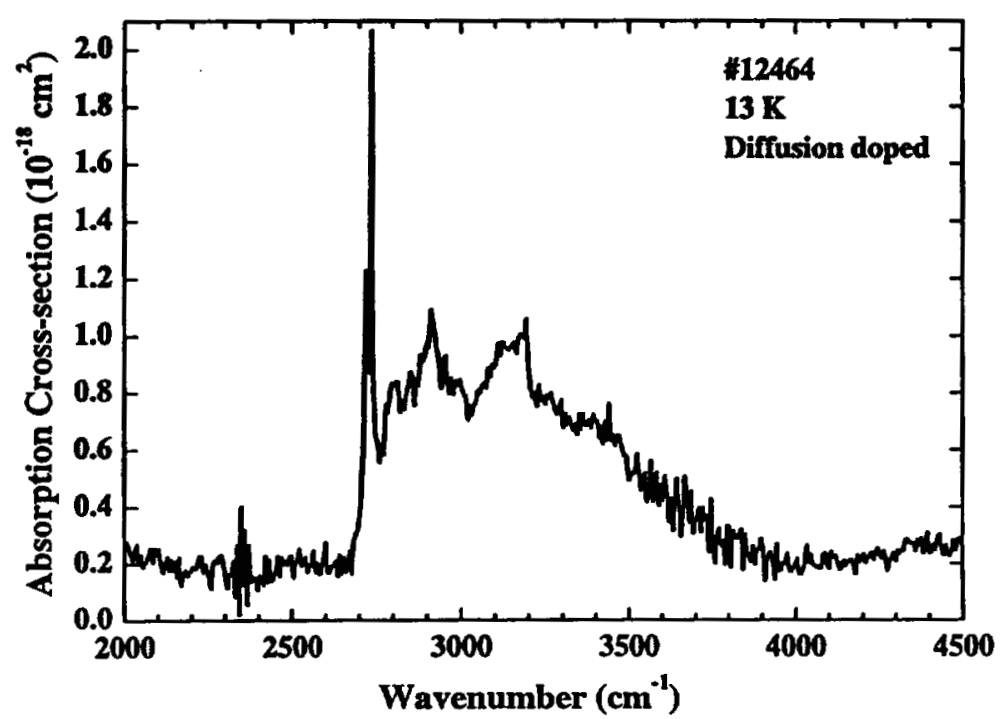

Figure 6.8. Unpolarized absorption spectrum at $13 \mathrm{~K}_{\text {for }} \mathrm{Fe}^{2+}: \mathrm{ZnSe}$ sample \#12464.

The features in Fig. 6.7 (zero-phonon lines and phonon sidebands) are the same as in Fig. 6.5. This means that $\mathrm{Fe}^{2+}$ was able to substitute for $\mathrm{Zn}^{2+}$ which suggests that 
diffusion doping is a very viable method to produce $\mathrm{Fe}^{2+}: \mathrm{ZnSe}$. Though the sample in Fig. 6.8 had a very low concentration of $\mathrm{Fe}$ (Table 6.3), its absorption features still very closely resemble those of Fig. 6.5 meaning that even the small amount of iron that did diffuse in substituted into $\mathrm{Zn}^{2+}$ sites. The spectra presented here for $\mathrm{Fe}^{2+}: \mathrm{ZnSe}$ suggest that an Er:YAG laser operating at $2.7 \mu \mathrm{m}\left(3703 \mathrm{~cm}^{-1}\right)$ would be a possible pump source for the emission and lifetime experiments.

\subsubsection{Temperature dependent emission spectra}

Unpolarized emission spectra were measured at different temperatures for $\mathrm{Fe}^{2+}: \mathrm{ZnSe}$. Fig. 6.9 shows the experimental setup used to measure the emission spectra at different temperatures. The Er:YAG laser operated at a wavelength of $2.698 \mu \mathrm{m}\left(3706 \mathrm{~cm}^{-1}\right)$ and delivered $48 \mu$ s long pulses at a repetition rate of $100 \mathrm{~Hz}$. The data was collected with a computer controlled 1 meter McPherson monochromator equipped with a $300 \mathrm{~g} / \mathrm{mm}$ grating, an EG\&G Judson liquid nitrogen cooled InSb detector, and a lock-in amplifier. A measured unpolarized emission spectrum at $34 \mathrm{~K}$ for $\mathrm{Fe}^{2+}: \mathrm{ZnSe}$ is shown in Fig. 6.10.

The measured emission spectra for $\mathrm{Fe}^{2+}: \mathrm{ZnSe}$ at different temperatures are presented in Fig. 6.12. The emission data has been corrected by the measured black-body response of the experimental apparatus and normalized to one. The peaks in the emission spectrum in Fig. 6.10 for wavenumbers $>2675 \mathrm{~cm}^{-1}$ are the zero phonon transitions and the broad band with wavenumbers $<2675 \mathrm{~cm}^{-1}$ is the phonon sideband. In Fig. 6.11, we include the absorption spectrum at $14 \mathrm{~K}$ that has been normalized to one to illustrate the relation between the emission and absorption wavelengths. Fig. 6.12 shows how the emission spectrum for $\mathrm{Fe}^{2+}: \mathrm{ZnSe}$ varies with temperature. The FWHM of the emission 


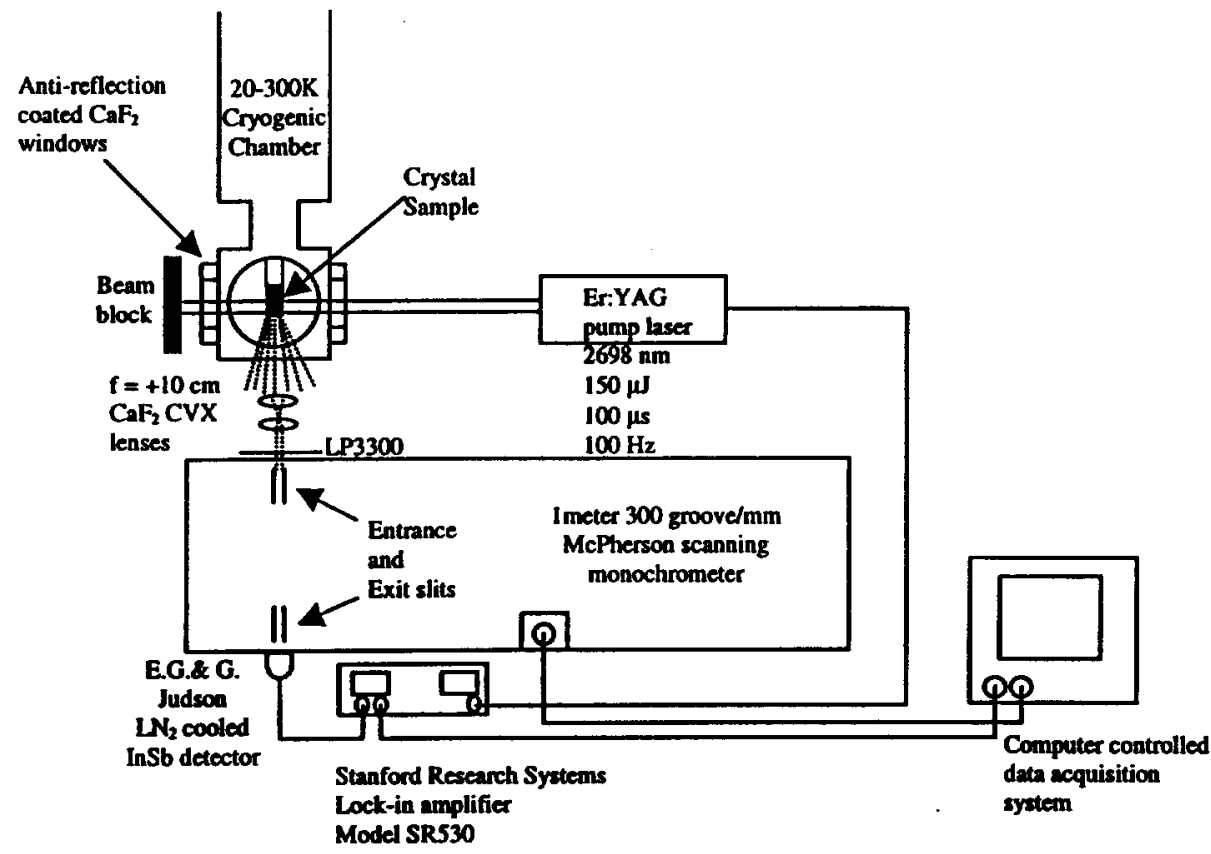

Figure 6.9. Experimental setup to measure emission spectra as a function of temperature.

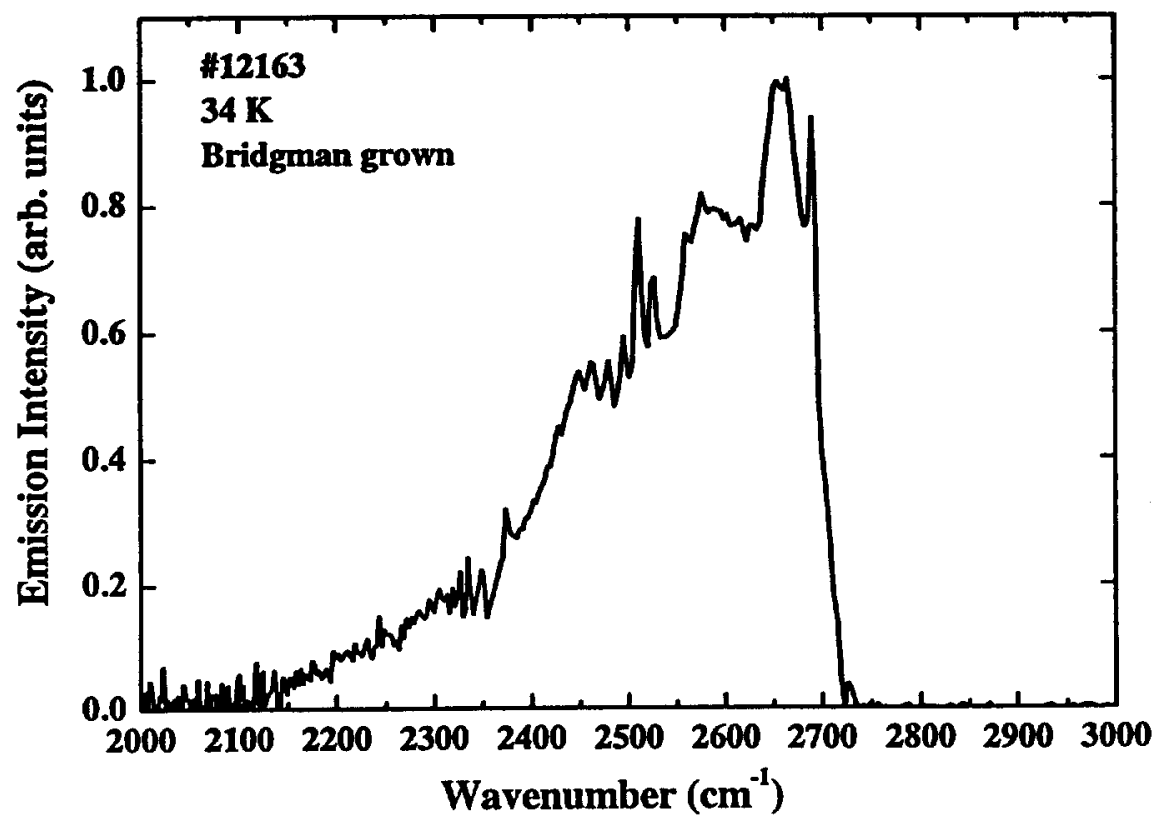

Figure 6.10. Unpolarized emission spectrum at $34 \mathrm{~K}$ for $\mathrm{Fe}^{2+}: \mathrm{ZnSe}$ sample \#12163. 


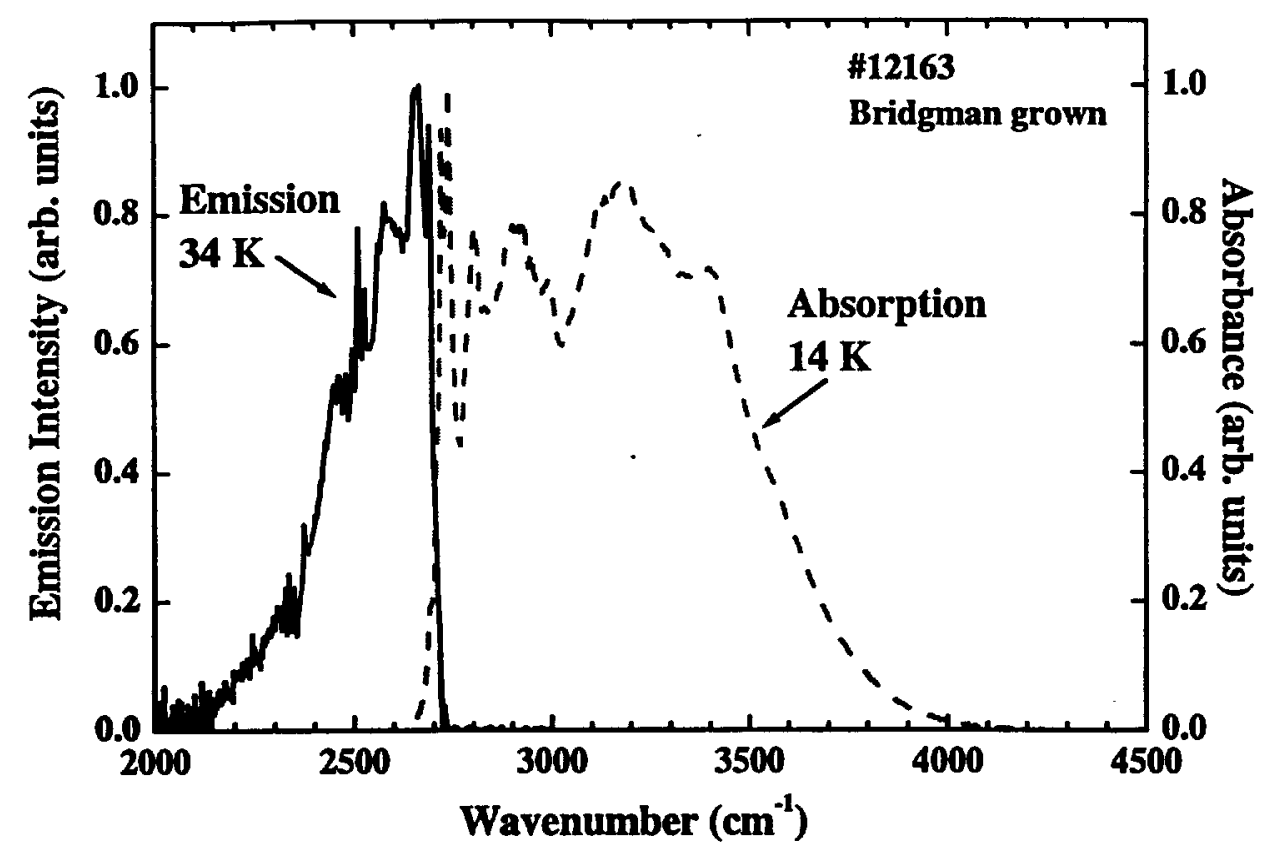

Figure 6.11. Low temperature unpolarized emission and absorption spectra for $\mathrm{Fe}^{2+}: \mathrm{ZnSe}$ sample \#12163.

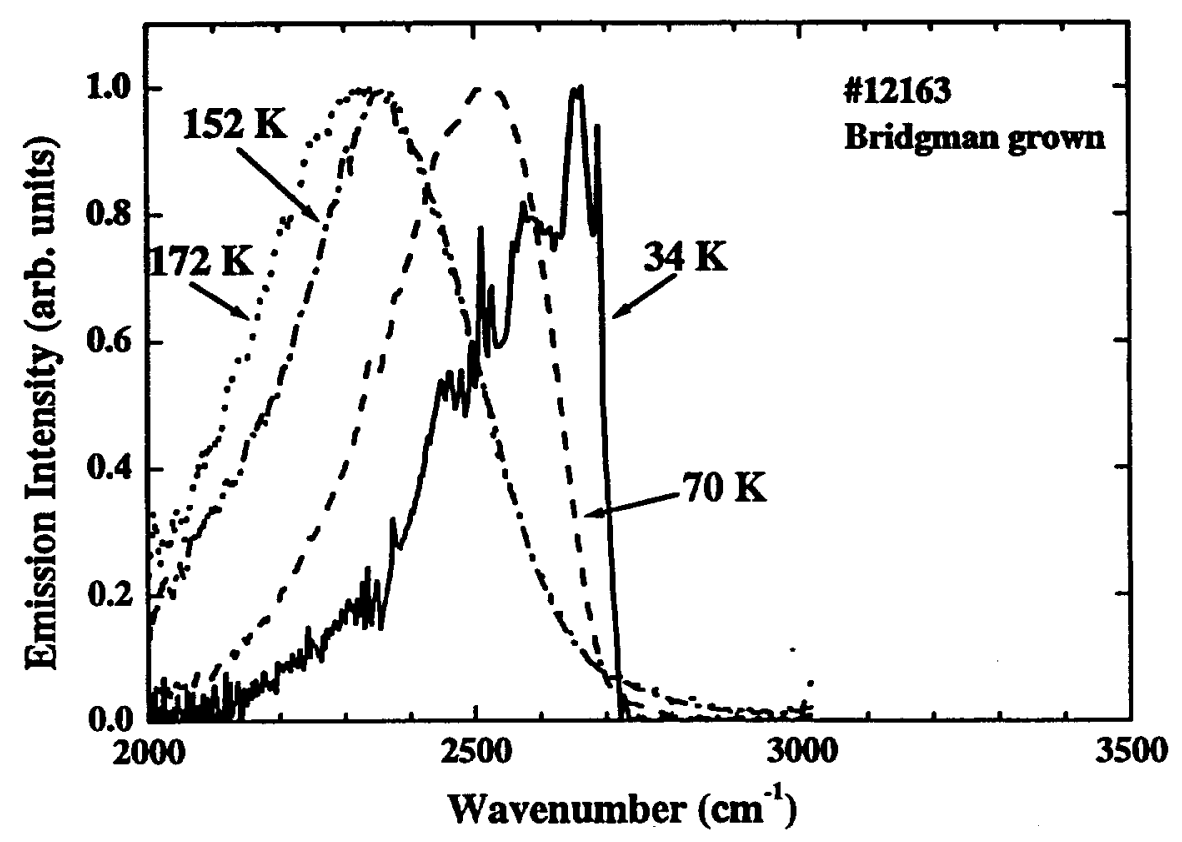

Figure 6.12. Unpolarized emission spectra at temperatures between $30 \mathrm{~K}$ and $180 \mathrm{~K}$ for $\mathrm{Fe}^{2+}: \mathrm{ZnSe}$ sample \#12163. 
spectra for $\mathrm{Fe}^{2+}: \mathrm{ZnSe}$ varies from about $300 \mathrm{~cm}^{-1}$ at $34 \mathrm{~K}$ to $500 \mathrm{~cm}^{-1}$ at $172 \mathrm{~K}$. This would suggest a potential tunability for $\mathrm{Fe}^{2+}: \mathrm{ZnSe}$ between $300 \mathrm{~cm}^{-1}$ to $500 \mathrm{~cm}^{-1}$ depending upon the temperature. We also note that above $225 \mathrm{~K}$ no luminescence was observed from $\mathrm{Fe}^{2+}: \mathrm{ZnSe}$.

\subsubsection{Temperature dependent luminescence lifetimes}

The luminescence lifetime data in Figs. $6.14-6.17$ was collected with the experimental setup shown in Fig. 6.13. In order to check reproducibility, the lifetime

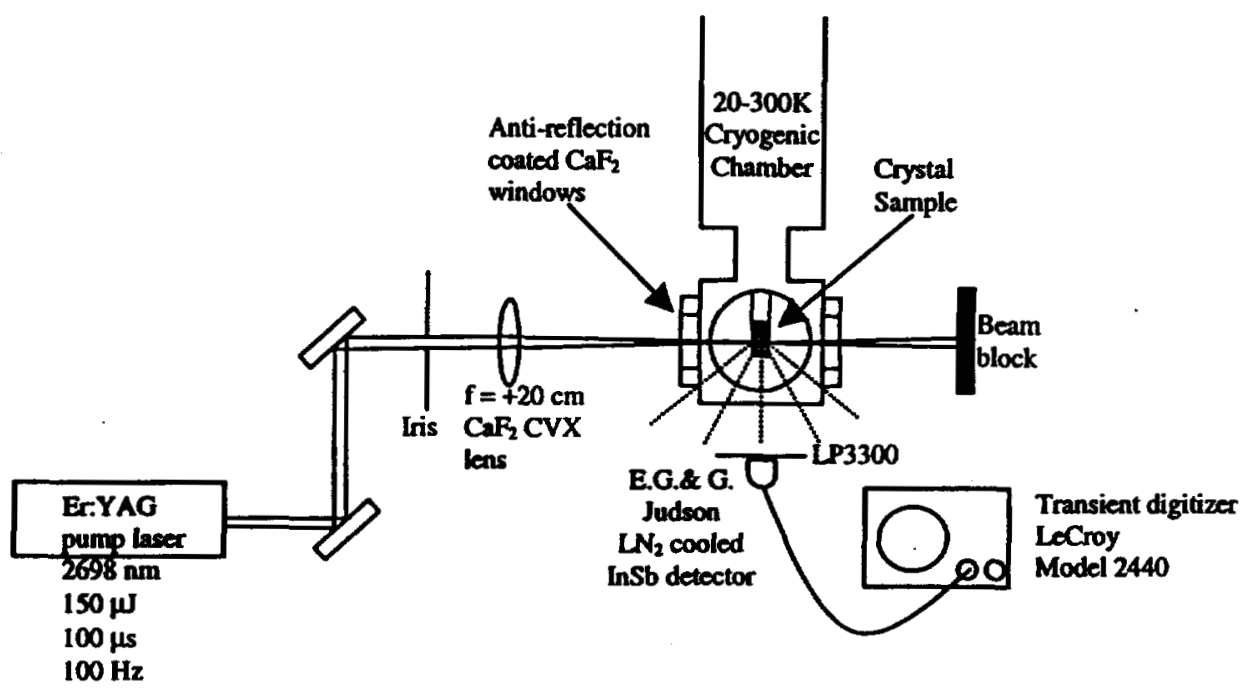

Figure 6.13. Experimental setup to measure the temperature dependent luminescence lifetimes.

data were taken several times and found to be repeatable to $\pm 5 \%$. The decline in lifetimes in Fig. 6.14 for temperatures between $100 \mathrm{~K}$ and $240 \mathrm{~K}$ is due to increased thermally-activated non-radiative decay described by Eqn. (6.15). The dramatic decline in lifetimes with decreasing temperature below $100 \mathrm{~K}$ was unexpected, however this same kind of effect has been seen much less dramatically for $\mathrm{Cr}^{2+}: \mathrm{ZnSe}$ and $\mathrm{Co}^{2+}: \mathrm{ZnSe}$ in Ref. [24]. No discussion of the effect was given in Ref. [24]. We suspect the effect is 


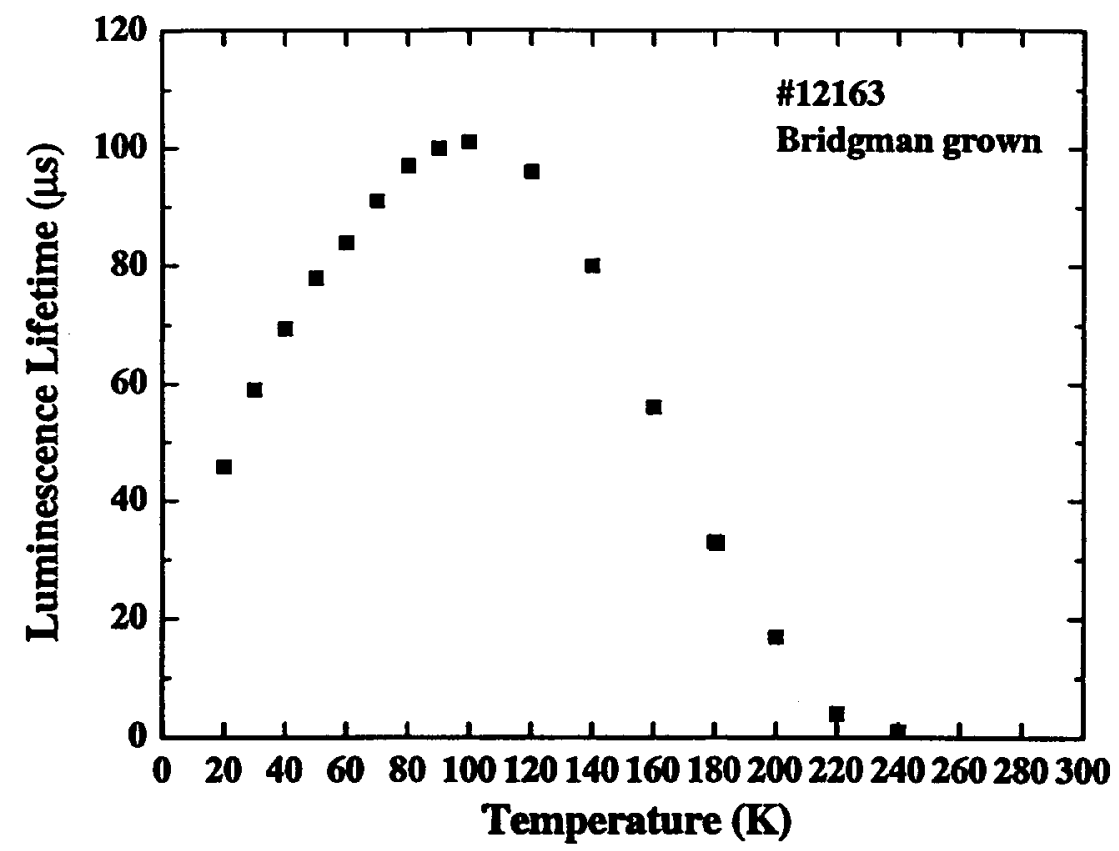

Figure 6.14. Luminescence lifetime as a function of temperature for $\mathrm{Fe}^{2+}: \mathrm{ZnSe}$ sample \#12163.

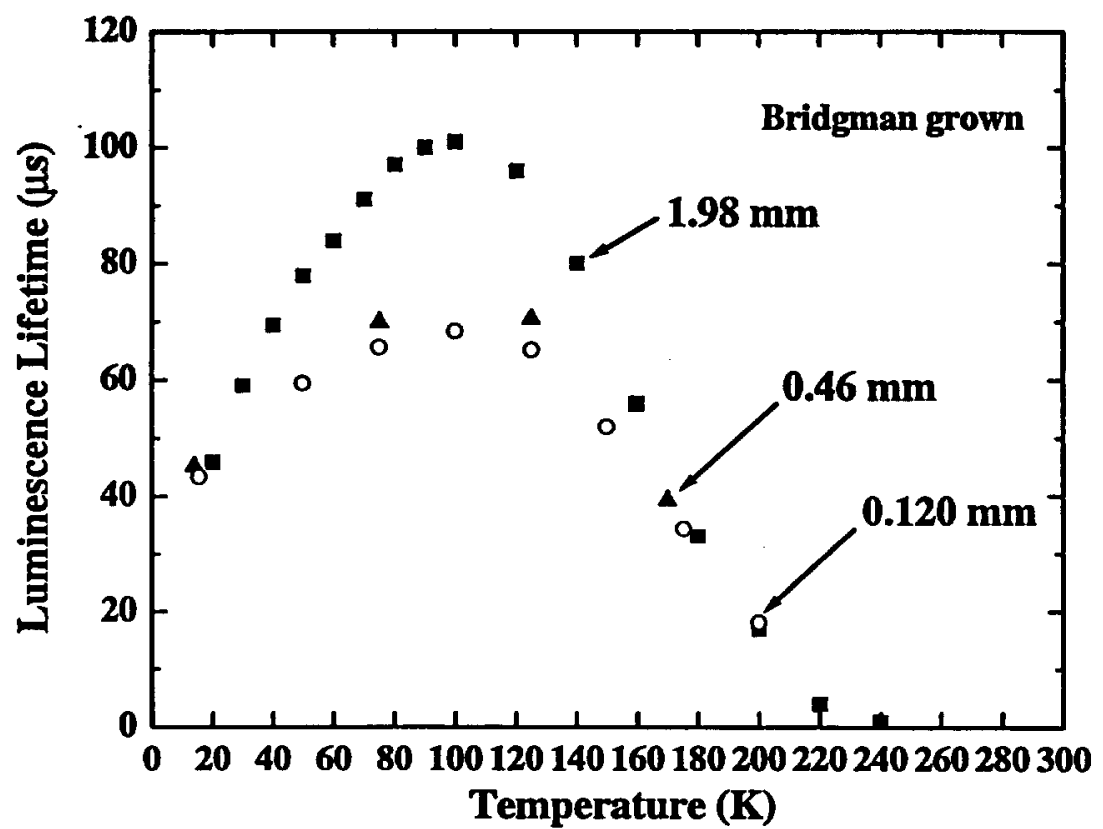

Figure 6.15. Luminescence lifetime as a function of temperature for $\mathrm{Fe}^{2+}: \mathrm{ZnSe}$ samples of different thicknesses (as shown). 
either due to thermal redistribution of the emitting population into levels with different radiative lifetimes or radiation trapping.

Radiation trapping [26] occurs when the neighboring ions to an emitting ion absorb and re-emit the photons causing the photons to random walk out of the material. The effect of the photon random walk is to increase the measured luminescence lifetime. This only occurs with three-level and quasi-three level transitions because under certain conditions (i.e. high enough temperature) there can be a thermal population in the terminal level to cause the re-absorption. The radiation trapping effect depends on three variables, the thermal population in the terminal level, the number density of emitting ions, and the physical size of the sample. A smaller sample will have less radiation trapping effects (i.e. a shorter measured lifetime) for a given level of doping since the photon random walk will be shorter. To see if the measured lifetime was affected by the sample size we performed measurements on samples of differing sizes. The data in Fig. 6.15 shows how the decreasing thickness of the samples can be seen to significantly reduce the variation of the luminescence lifetimes for temperatures between $14 \mathrm{~K}$ and $110 \mathrm{~K}$. Therefore we conclude that the decrease in measured lifetime for temperatures below $100 \mathrm{~K}$ is primarily due to radiation trapping.

Figs. 6.16 and 6.17 show the luminescence lifetimes measured for the diffusion doped samples. The diffusion doped samples have the same approximate values for the luminescence lifetime and behavior with temperature as the Bridgman grown samples. The sample in Fig. 6.17 has very low Fe concentration (Table 6.3). So in this case, the effect of radiation trapping is decreased through the reduced concentration of $\mathrm{Fe}^{2+}$. The 


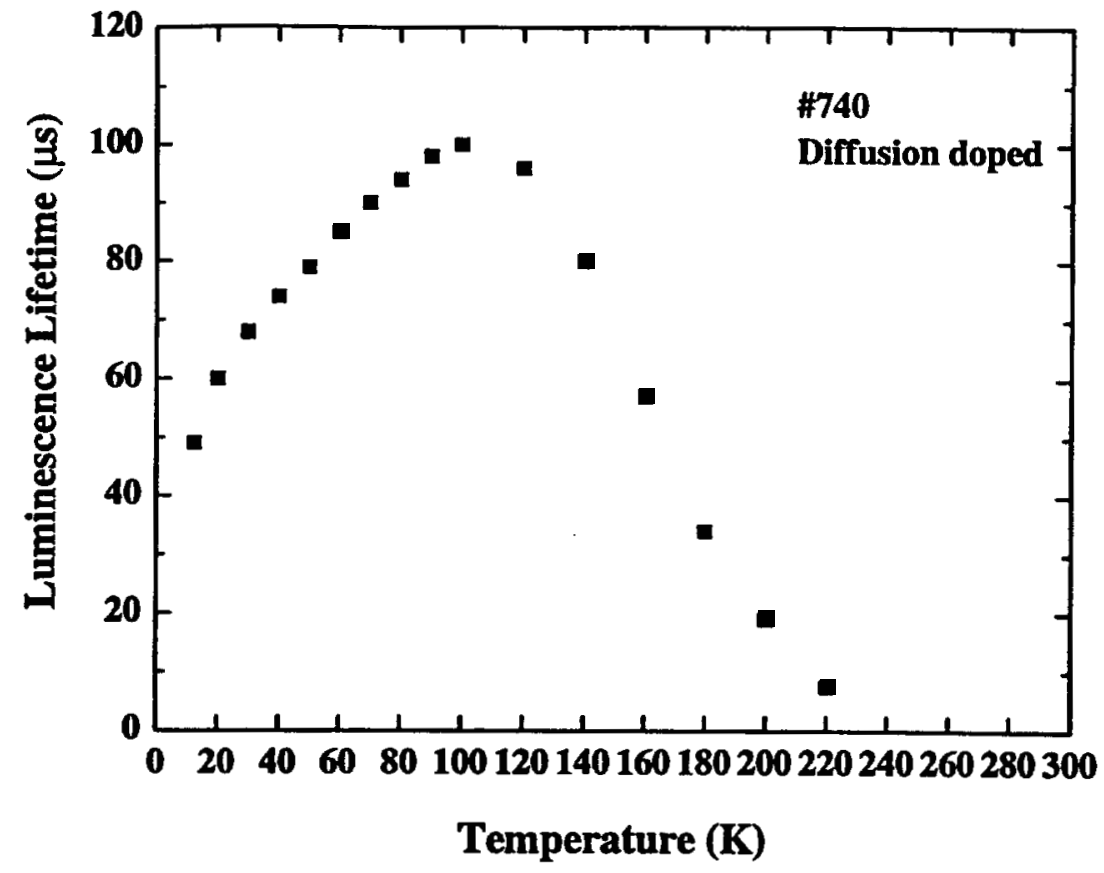

Figure 6.16. Luminescence lifetime as a function of temperature for $\mathrm{Fe}^{2+}: \mathrm{ZnSe}$ sample \#740.

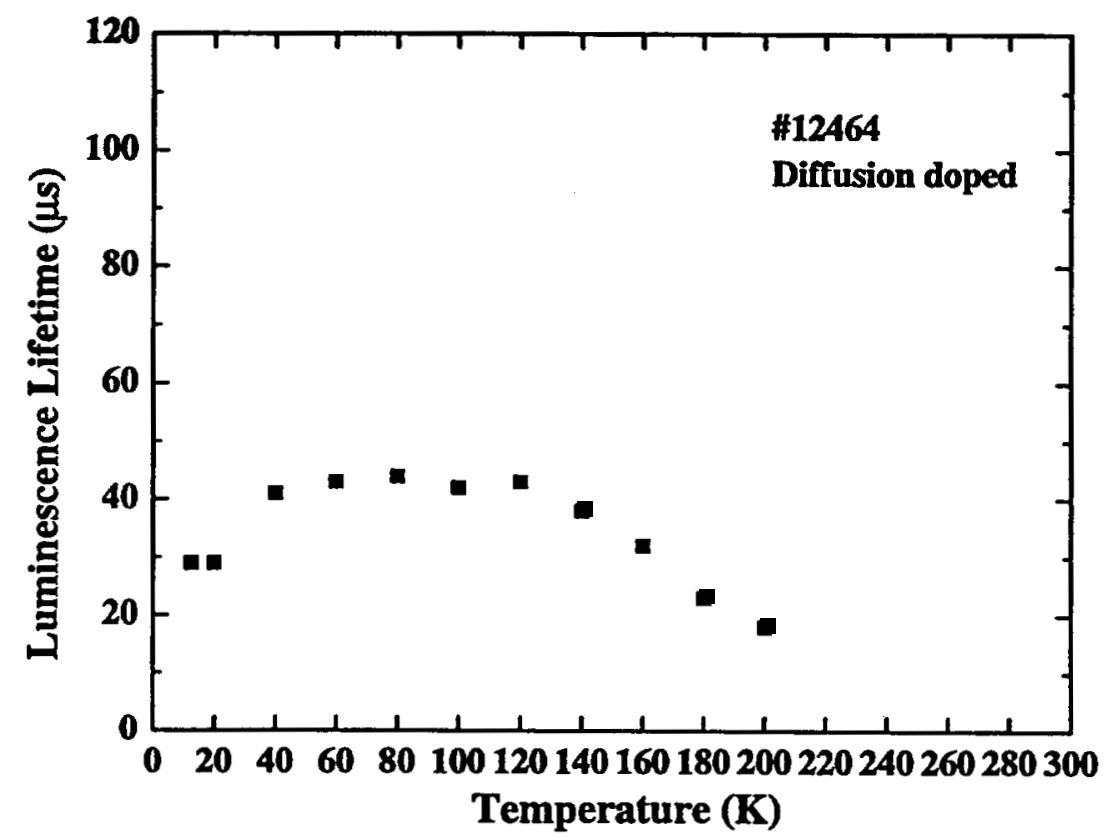

Figure 6.17. Luminescence lifetime as a function of temperature for $\mathrm{Fe}^{2+}: \mathrm{ZnSe}$ sample \#12464. 
reduced concentration of $\mathrm{Fe}^{2+}$ means there are spatially fewer ions to reabsorb the emitted photons and therefore the random walk of the photons is shorter.

\subsection{Analysis and discussion}

\subsubsection{Emission cross-sections}

The emission spectra in Fig. 6.12 were converted to cross-section using the Einstein relation [40]

$$
\sigma_{\mathrm{em}}(\lambda)=\frac{\lambda^{5} \mathrm{I}(\lambda)}{8 \pi \mathrm{cn}^{2} \tau_{\mathrm{rad}} \int_{0}^{\infty} \mathrm{I}(\lambda) \lambda \mathrm{d} \lambda}
$$

where $\mathrm{n}=$ refractive index for $\mathrm{ZnSe}=2.4, \mathrm{I}(\lambda)$ is the blackbody-calibrated emission spectrum, and $\tau_{\text {rad }}$ is the radiative lifetime of the transition. The radiative lifetime was taken as the luminescence lifetime at $20 \mathrm{~K}(49 \mu \mathrm{s})$ in Fig. 6.14. At $20 \mathrm{~K}$, radiation trapping effects and thermal non-radiative processes should be at a minimum, therefore it is reasonable to assume that the luminescence lifetime at $20 \mathrm{~K}$ would most closely resemble the radiative lifetime. Therefore $49 \mu$ s will serve as our best estimate of the radiative lifetime. Fig. 6.18 shows the converted emission spectra for $\mathrm{Fe}^{2+}: \mathrm{ZnSe}$ in terms of cross-section. 


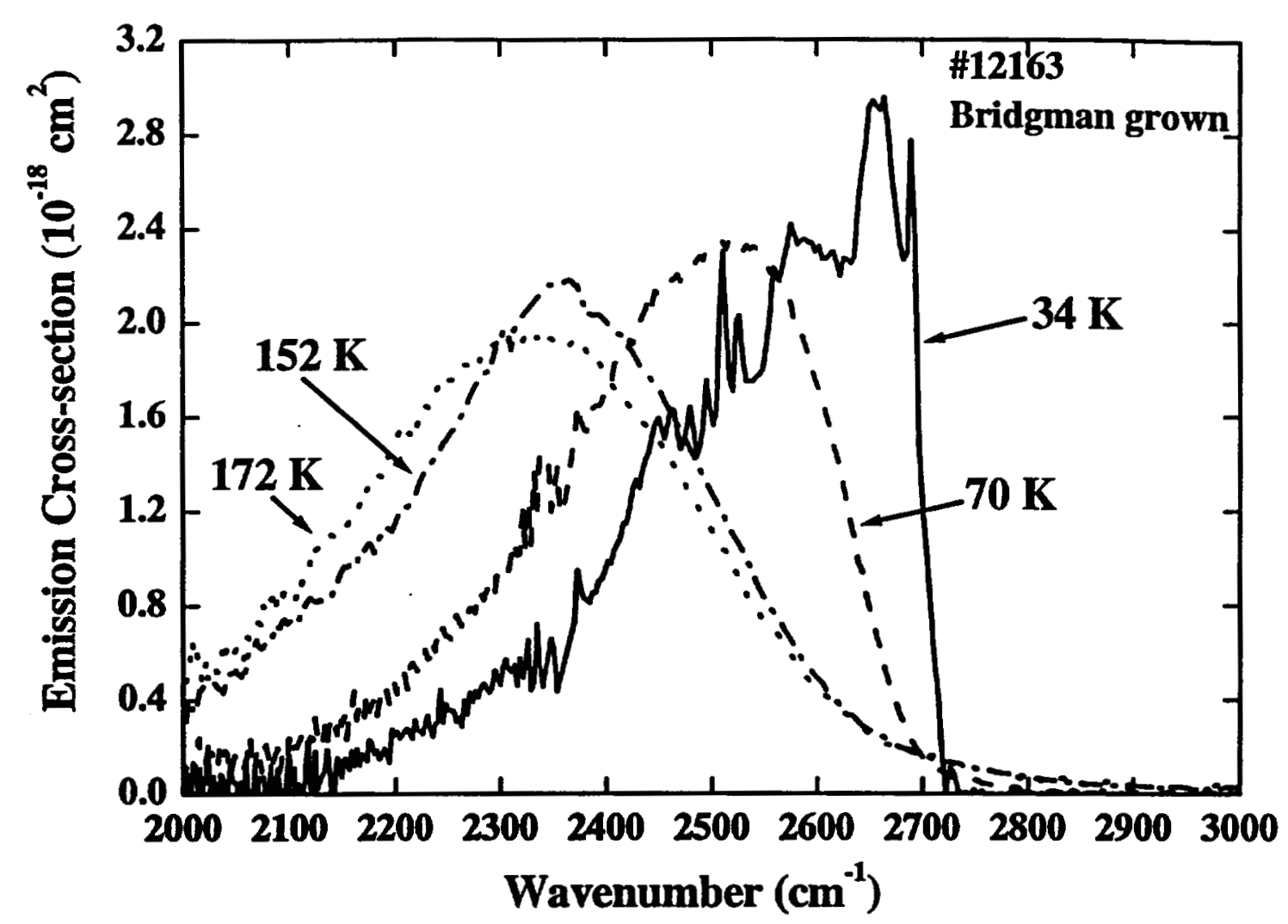

Figure 6.18. Unpolarized emission cross-sections at temperatures between $30 \mathrm{~K}$ and 180 $\mathrm{K}$ for $\mathrm{Fe}^{2+}: \mathrm{ZnSe}$ sample \#12163.

\subsubsection{Zero-phonon lines}

In Fig. 6.19 we show an expanded view of the absorption spectrum from Fig. 6.5 with several zero-phonon lines labeled. The notation $E_{(i-j)}$ follows that discussed for Eqns. (6.5) - (6.9) except for $E_{(1-7)}$, which designates the energy difference between levels 1 and 7 in Fig. 6.2. Our labeling of the particular features in Fig. 6.19 in general follows that of Ref. [20] and [29]. The decrease in cross-section of lines $E_{(1-6)}$ through $E_{(5-6)}$ reflects the thermal distribution (Boltzmann) of population over the lower levels. Table 6.4 lists the lines identified, their corresponding energies, and the results from Ref. [29] for comparison. The energy values for the first four lines agree within experimental errors 


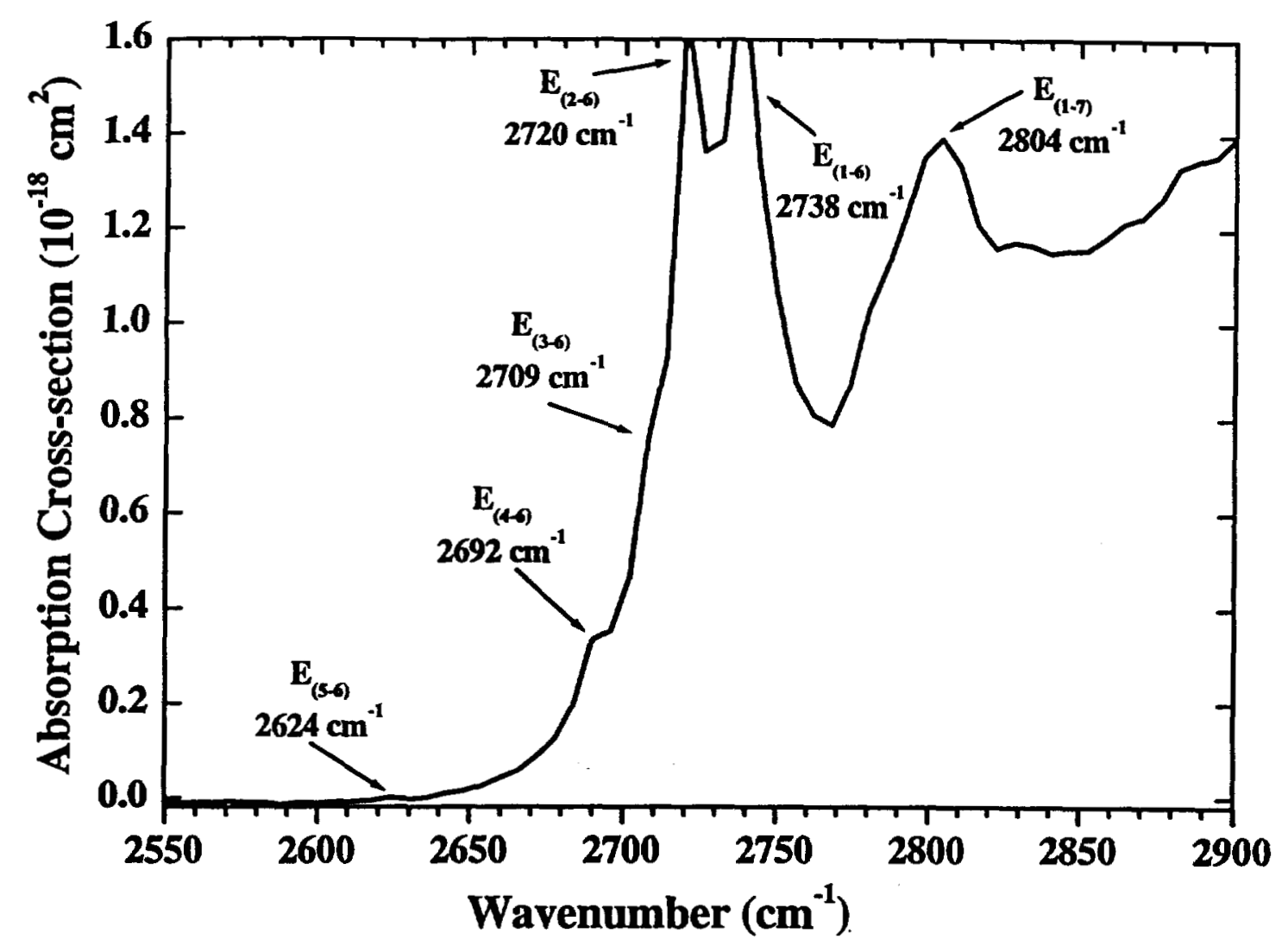

Figure 6.19. Expanded view of the $14 \mathrm{~K}$ absorption spectrum in Fig. 6.5 with several zero-phonon lines labeled.

of those found by Ref. [29]. $E_{(5-6)}$ and $E_{(1-7)}$ were not identified in Ref. [29]. Our identification of the feature at $2624 \mathrm{~cm}^{-1}$ as corresponding to $E_{(5-6)}$ may not be correct. The $5 \rightarrow 6$ transition is symmetry forbidden and level 5 in the lower manifold would have very low $\left(<10^{-5}\right)$ fractional thermal population at $14 \mathrm{~K}$. These two factors would make the intensity of the $\mathrm{E}_{(5-6)}$ line virtually imperceivable in the spectrum and therefore very difficult to identify relative to the other lines that have fractional populations $z 10^{-2}$. The peak at $2804 \mathrm{~cm}^{-1}$ was assigned as $E_{(1-7)}$ because it is relatively narrow. However, the strength (i.e. cross-section value) of the line makes the assignment suspect because the $1 \rightarrow 7$ transition is symmetry forbidden. Further consideration is needed to confidently regard the feature as $E_{(1-7)}$. Note that the spacing between the first 4 lines in Table 6.4 is 
Table 6.4. Energies of several zero-phonon lines for the ${ }^{5} \mathrm{E} \rightarrow{ }^{5} \mathrm{~T}_{2}$ transition in $\mathrm{Fe}^{2+}: \mathrm{ZnSe}$.

\begin{tabular}{|c|c|c|}
\hline Transition & $\begin{array}{c}\text { Energy } \\
\left(\mathrm{cm}^{-1}\right)\end{array}$ & $\begin{array}{c}\text { Baranowski et al. [11] } \\
\left(\mathrm{cm}^{-1}\right)\end{array}$ \\
\hline $\mathbf{E}_{(1-6)}$ & $2738 \pm 2$ & 2738 \\
\hline $\mathbf{E}_{(2-6)}$ & $2720 \pm 2$ & 2721 \\
\hline $\mathbf{E}_{(3-6)}$ & $2708 \pm 4$ & 2710 \\
\hline $\mathbf{E}_{(4-6)}$ & $2692 \pm 4$ & 2695 \\
\hline $\mathbf{E}_{(5-6)}$ & $2624 \pm 8$ & - \\
\hline $\mathbf{E}_{(1-7)}$ & $2804 \pm 5$ & - \\
\hline
\end{tabular}

relatively constant $\left(\sim 15 \mathrm{~cm}^{-1}\right)$ which agrees with what can be predicted from Eqns. (6.5) (6.8). We would also expect lines $\mathrm{E}_{(4-6)}$ and $\mathrm{E}_{(5-6)}$ to be spaced by the same amount. However, from Table 6.4 we find that the spacing is $68 \mathrm{~cm}^{-1}$. This discrepancy is most likely due once again to the difficulty in properly assigning the $\mathrm{E}_{(5-6)}$ line in the spectrum.

\subsubsection{Lattice phonon energies}

We will use the phonon sideband in the low temperature absorption spectrum to estimate a characteristic phonon energy for $\mathrm{Fe}^{2+}: \mathrm{ZnSe}$. We are assuming for simplicity that the sideband peaks are due to coupling to a single vibrational mode, which is not exactly the case since several modes are most likely participating in the coupling [35], however we are simply trying to estimate a consistent value for a characteristic phonon energy. In Fig. 6.20 we label the peaks in the absorption spectrum that we identify as $\mathrm{E}_{(1-}$ 6) combined with one lattice phonon of energy $\hbar \omega_{\text {peak } A}=182 \pm 34 \mathrm{~cm}^{-1}$ and $E_{(1-6)}$ combined with two lattice phonons each with energy $\hbar \omega_{\text {peak }}$ B $=225.5 \pm 25 \mathrm{~cm}^{-1}$ (i.e. $2 \mathrm{x}$ $225.5 \mathrm{~cm}^{-1}=451 \mathrm{~cm}^{-1}$. Note that we choose here to regard this as coupling to two phonons rather than a single phonon with energy $451 \mathrm{~cm}^{-1}$ because $\mathrm{ZnSe}$ has phonon frequencies that are $s 300 \mathrm{~cm}^{-1}$ [41].). The uncertainties were found by estimating the 
widths of the peaks used to determine the phonon energies. Note that the values for $\hbar \omega_{\text {peak }}$ A and $\hbar \omega_{\text {peak }}$ agree within the experimental uncertainty, which may imply that the two peaks are coupling to the same vibrational mode.

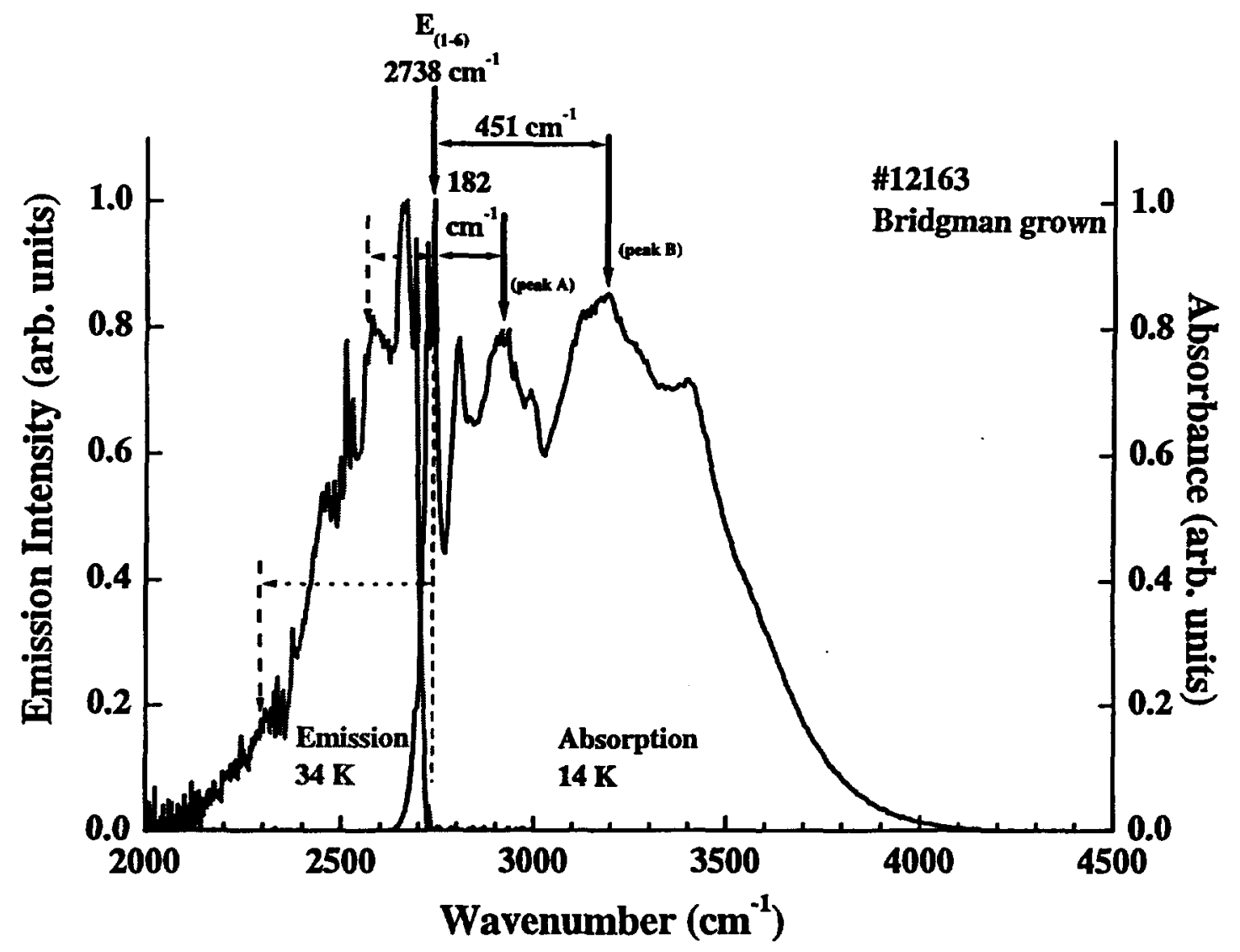

Figure 6.20. Low temperature unpolarized emission and absorption spectra for $\mathrm{Fe}^{2+}: \mathrm{ZnSe}$ with the dominate sideband transitions in the absorption spectrum labeled.

We also label in Fig. 6.20 (dashed arrows) where the corresponding peaks in the emission spectrum would appear for this same characteristic phonon energy. Note that at $E_{(1-6)}-182 \mathrm{~cm}^{-1}$ and $E_{(1-6)}-451 \mathrm{~cm}^{-1}$ there does not appear to be distinct corresponding peaks in the emission spectrum. We would expect the excited state to have smaller phonon energies than the ground state [35] since the excited state has a larger inter-ion distance (between the $\mathrm{Fe}^{2+}$ and $\mathrm{Se}^{2-}$ ions) which results in a lower bond strength and 
therefore a lower bond vibrational energy (i.e. lower phonon energies). Therefore the lack of corresponding peaks in the emission spectrum is not physically unreasonable. In Table 6.5, we compare the phonon energies found from Fig. 6.20 to values determined by Hennion, et al. for $\mathrm{ZnSe}$ [41]. Hennion, et al. measured the energies for the longitudinaloptical (LO) and transverse-optical (TO) phonons in $\mathrm{ZnSe}$ using inelastic neutron scattering [42] and found good agreement with values from other researchers. Inelastic scattering would yield phonon energies in the ground state, which would imply that, the phonon energies determined by Hennion, et al. should be larger than the values

Table 6.5. Lattice phonon energies for $\mathrm{Fe}^{2+}: \mathrm{ZnSe}$ and $\mathrm{ZnSe}$.

\begin{tabular}{|c|c|}
\hline $\begin{array}{c}\text { Phonon energies for Fe } \\
\text { the absorption spectrum in this work } \\
\left(\mathrm{cm}^{-1}\right)\end{array}$ & $\begin{array}{c}\text { Phonon energies for ZnSe } \\
\text { found by Hennion et al. [24] } \\
\left(\mathrm{cm}^{-1}\right)\end{array}$ \\
\hline$\hbar \omega_{\text {peak A }}=182 \pm 30 \quad \hbar \omega_{\text {penk B }}=225 \pm 25$ & $\hbar \omega_{\mathrm{TO}}=213$ \\
& $\hbar \omega_{L \mathrm{LO}}=253$ \\
\hline
\end{tabular}

TO $=$ transverse-optical and LO = longitudinal-optical .

determined here from the absorption spectrum (which measures the phonon energies in the excited state). Upon examination of the values in Table 6.5, we see that the phonon energies determined here from the absorption spectrum are indeed lower in value than those determined by Hennion, et al. which is consistent with what we physically expect.

\subsubsection{Tanabe-Sugano diagram}

Combining the energies of zero-phonon lines $\mathrm{E}_{(1-6)}$ and $\mathrm{E}_{(2-6)}$ given in Table 6.4 with Eqns. (6.5) and (6.6), we can calculate values for the crystal field strength parameter ( $\Delta$ ) and the spin-orbit interaction parameter $(\lambda)$. We choose to use $E_{(1-6)}$ and $E_{(2-6)}$ in the 
calculation because we know these energies most accurately. Table 6.6 lists the calculated values for $\Delta$ and $\lambda$. The value for $\lambda$ of $-94 \mathrm{~cm}^{-1}$ is approximately $10 \%$ smaller than the $\mathrm{Fe}^{2+}$ free-ion value of $-103 \mathrm{~cm}^{-1}$ [29]. This reduction in the value for $\lambda$ from the free-ion value occurs because the interaction with the crystal field has modified the electrons' wavefunctions, which when used to evaluate the matrix elements of the Hamiltonian involving the spin-orbit operator results in a lower spin-orbit interaction strength (i.e. smaller magnitude for $\lambda$ ) [26]. The negative sign for $\lambda$ is expected because a $d^{6}$ ion has a more than half-filled $d-$ shell [43].

Table 6.6. Calculated crystal field strength and spin-orbit interaction parameters.

\begin{tabular}{|c|c|}
\hline Parameter & Calculated value \\
\hline$\Delta$ & $2937 \pm 200 \mathrm{~cm}^{-1}$ \\
\hline$\lambda$ & $-94 \pm 8 \mathrm{~cm}^{-1}$ \\
\hline
\end{tabular}

Using $\Delta$ from Table 6.6 and the $\mathrm{Fe}^{2+}$ free-ion value for the Racah B parameter $(917$ $\mathrm{cm}^{-1}$ ), we can determine $\triangle \mathrm{BB}$ and $\mathrm{E} / \mathrm{B}$ for $\mathrm{Fe}^{2+}: \mathrm{ZnSe}$ on the Tanabe-Sugano diagram. The value calculated for $\Delta / B$ is 3.2. Since the line that represents the ${ }^{5} T_{2}$ state in Fig. 6.21 has unity slope in the range $\Delta / B<27, \Delta / B=3.2$ implies $E / B=3.2$. The position $E / B=3.2$ and $\Delta \mathrm{B}=3.2$ is labeled in Fig. 6.21. Given the energy of the ${ }^{5} \mathrm{~T}_{2}$ state in $\mathrm{Fe}^{2+}: \mathrm{ZnSe}$ as shown on the Tanabe-Sugano diagram, the next higher state is $-20,000 \mathrm{~cm}^{-1}$ above it. Therefore, excited state absorption in $\mathrm{Fe}^{2+}: \mathrm{ZnSe}$ will be improbable because of the difference in spin multiplicity and the large difference in energy between the higher lying states and the ${ }^{5} \mathrm{~T}_{2}$ state. 


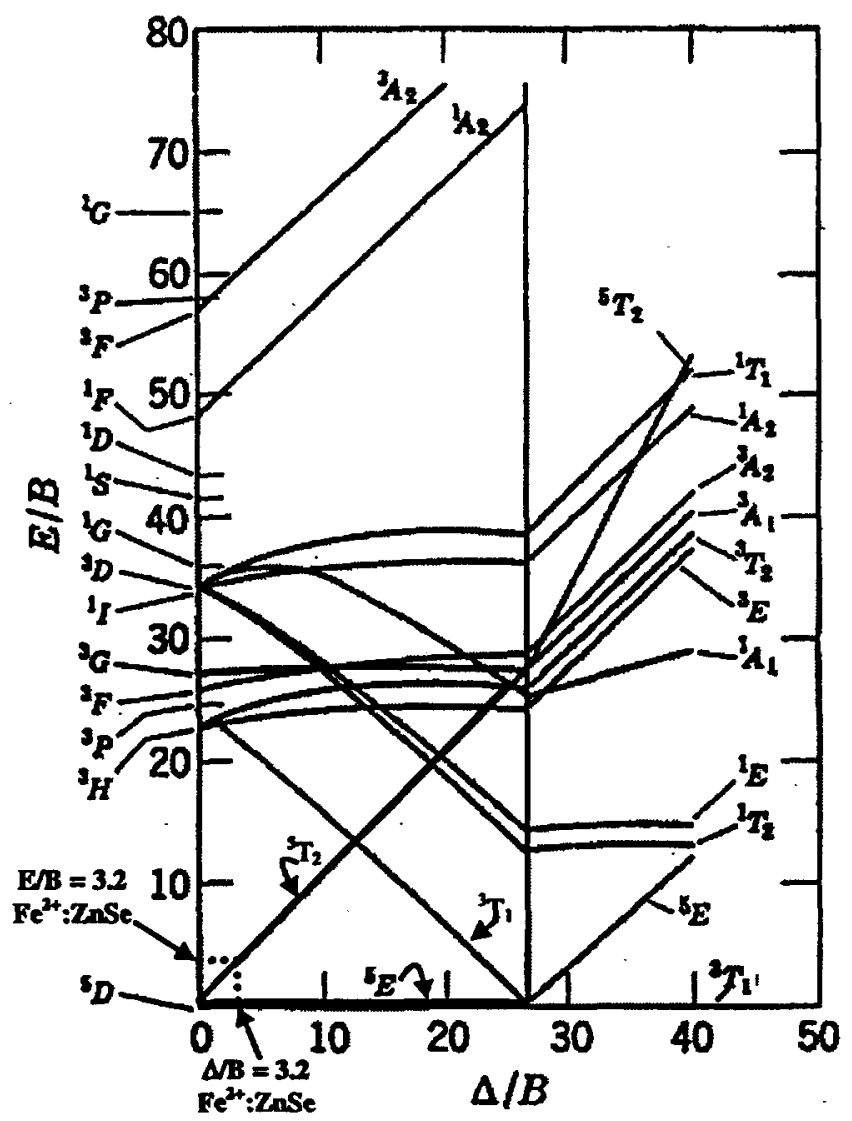

Figure 6.21. Tanabe-Sugano diagram for a $\mathrm{d}^{6}$ ion in a tetrahedral field showing $\Delta / \mathrm{B}$ and $\mathrm{E} / \mathrm{B}$ for $\mathrm{Fe}^{2+}: \mathrm{ZnSe}$ determined in this work.

\subsubsection{Huang-Rhys parameter and Condon offset}

An estimate for the Huang-Rhys parameter (S) can be determined by using the result in section 6.4 .3 that the peak in the absorption spectrum at $E_{(1-6)}+451 \mathrm{~cm}^{-1}$ was due to coupling to 2 phonons. Using this information combined with Eqn. (6.18) (i.e. $\mathrm{m}=2$ ) and Eqn. (6.19) we find $S=2.5$. Another way to estimate the Huang-Rhys parameter is to note, as Henderson and Imbush do [30], that at $T=0 \mathrm{~K}$, the area under the zerophonon lines in the absorption spectrum is given by $\mathrm{I}_{0} \mathrm{e}^{-\mathrm{s}}$ where $\mathrm{I}_{\mathrm{o}}$ is the area under the entire absorption spectrum. Therefore the Huang-Rhys parameter can be estimated using 


$$
\mathrm{e}^{-\mathrm{S}} \cong \frac{\text { Area under the zero phonon lines in the } 14 \mathrm{~K} \text { absorption spectrum }}{\text { Area under the entire } 14 \mathrm{~K} \text { absorption spectrum }}
$$

Calculating in a spreadsheet the areas from the absorption spectrum at $14 \mathrm{~K}$ in Fig. 6.5 yields,

$$
\mathrm{e}^{-\mathrm{S}}=\frac{83.4 \pm 30 \mathrm{~cm}}{1162.2 \mathrm{~cm}}=0.072 \pm 0.026
$$

giving $\mathrm{S} \cong 2.6 \pm 0.4$ for $\mathrm{Fe}^{2+}: \mathrm{ZnSe}$. This compares very well with the value of $S=2.5$ calculated from $\mathrm{E}_{\text {dis. }}$. These values for $\mathrm{S}$ imply that the coupling between the $\mathrm{Fe}^{2+}$ ions and the $\mathrm{ZnSe}$ lattice is in the weak coupling regime as discussed in section 6.2.2.

The Condon offset $(\mathrm{Q}(\mathrm{e})-\mathrm{Q}(\mathrm{g}))$ can now be calculated from Eqn. (6.20). Substituting $S=2.5 \pm 0.4, M=$ mass of a Se atom, and $\hbar \omega_{0}=\hbar \omega_{\text {peak } B}=225 \pm 30 \mathrm{~cm}^{-1}$ in Eqn. (6.20) we find $(Q(e)-Q(g)) \cong 0.0065 \pm 0.0019 \AA$. This estimate for the Condon offset will be used as a guide to construct the configurational coordinate diagram for the ${ }^{5} \mathrm{~T}_{2}$ and ${ }^{5} \mathrm{E}$ states in $\mathrm{Fe}^{2+}: \mathrm{ZnSe}$.

\subsubsection{Thermal activation energy, $E_{A}$}

The thermal activation energy $\left(E_{A}\right)$ can be estimated using Eqn. (6.21) with $E_{\text {ZPL }}=$ $\mathrm{E}_{(1-6)}=2738 \pm 2 \mathrm{~cm}^{-1}, \mathrm{~S}=2.5 \pm 0.4$, and $\hbar \omega_{0}=\hbar \omega_{\text {peak } \mathrm{B}}=225 \pm 30 \mathrm{~cm}^{-1}$. From Eqn. (6.21) we find $E_{A}=2104 \pm 750 \mathrm{~cm}^{-1}$. Another method of determining $E_{A}$ is by fitting Eqn. (6.17) to the lifetime data presented in section 6.3.4 [34]. Of the three sets of data analyzed the data in Fig. 6.14 allowed the best fit to Eqn. (6.17). From the fit, we were able to extract values for the radiative transition rate $\left(W_{\text {radiative }}\right)$, the high temperature 
thermal nonradiative transition rate $\left(\mathrm{W}_{\mathrm{nr}}^{\mathrm{o}}\right)$, and $\mathrm{E}_{\mathrm{A}}$. Table 6.7 lists the values obtained from the fit.

Table 6.7. Values resulting from fitting Eqn. (6.17) to the lifetime data in Fig. 6.14.

\begin{tabular}{|c|c|}
\hline $\begin{array}{c}\text { Lifetime fit } \\
\text { parameter }\end{array}$ & Value \\
\hline $\mathbf{W}_{\text {radiative }}$ & $13714 \mathrm{~s}^{-1}$ \\
\hline $\mathbf{W}_{\mathrm{nr}}^{0}$ & $1.44 \times 10^{12} \mathrm{~s}^{-1}$ \\
\hline $\mathbf{E}_{\mathbf{A}}$ & $2171 \mathrm{~cm}^{-1}$ \\
\hline
\end{tabular}

The radiative transition rate $\left(\mathrm{W}_{\text {radiative }}\right)$ can be inverted to give a value for the radiative lifetime of $73 \mu \mathrm{s}$. This value falls within the range of values measured for the luminescence lifetime below $200 \mathrm{~K}$. Note that $73 \mu \mathrm{s}$ is about twice the value we assumed for the radiative lifetime in converting the emission spectra to cross-section. This would imply that our assumption for the magnitude of the radiative lifetime is at least within the correct order of magnitude. $\mathrm{W}_{\mathrm{nr}}^{0}$ is the same order of magnitude as other values found in the literature [34]. The value for $E_{A}$ determined using Eqn. (6.21) and the value in Table 6.7 show good agreement. The two values will be averaged and the average value will be used in the construction of the configurational coordinate diagram. Averaging the two values for $E_{A}\left(2104\right.$ and $\left.2171 \mathrm{~cm}^{-1}\right)$ gives $E_{A}^{2 v g}=2138 \mathrm{~cm}^{-1}$.

\subsubsection{Configurational coordinate diagram}

The values found in the previous sections for the zero-phonon lines, the lattice phonon energy ( $\left.\hbar \omega_{\text {peak }} \mathrm{B}\right)$, the Condon offset $(\mathrm{Q}(\mathrm{e})-\mathrm{Q}(\mathrm{g}))$, and the thermal activation energy $\left(E_{A}=E_{A}{ }^{\text {avg }}\right)$ were used to construct the scaled configurational coordinate diagram 
for $\mathrm{Fe}^{2+}: \mathrm{ZnSe}$ shown in Fig. 6.22. In constructing the diagram, we assumed for simplicity that the ${ }^{5} \mathrm{E}$ and ${ }^{5} \mathrm{~T}_{2}$ states have equal phonon energies of $225 \mathrm{~cm}^{-1}$. This was the value we determined explicitly from the absorption spectrum, however the assumption that phonons in the ${ }^{5} \mathrm{E}$ state have this energy was neither proved nor

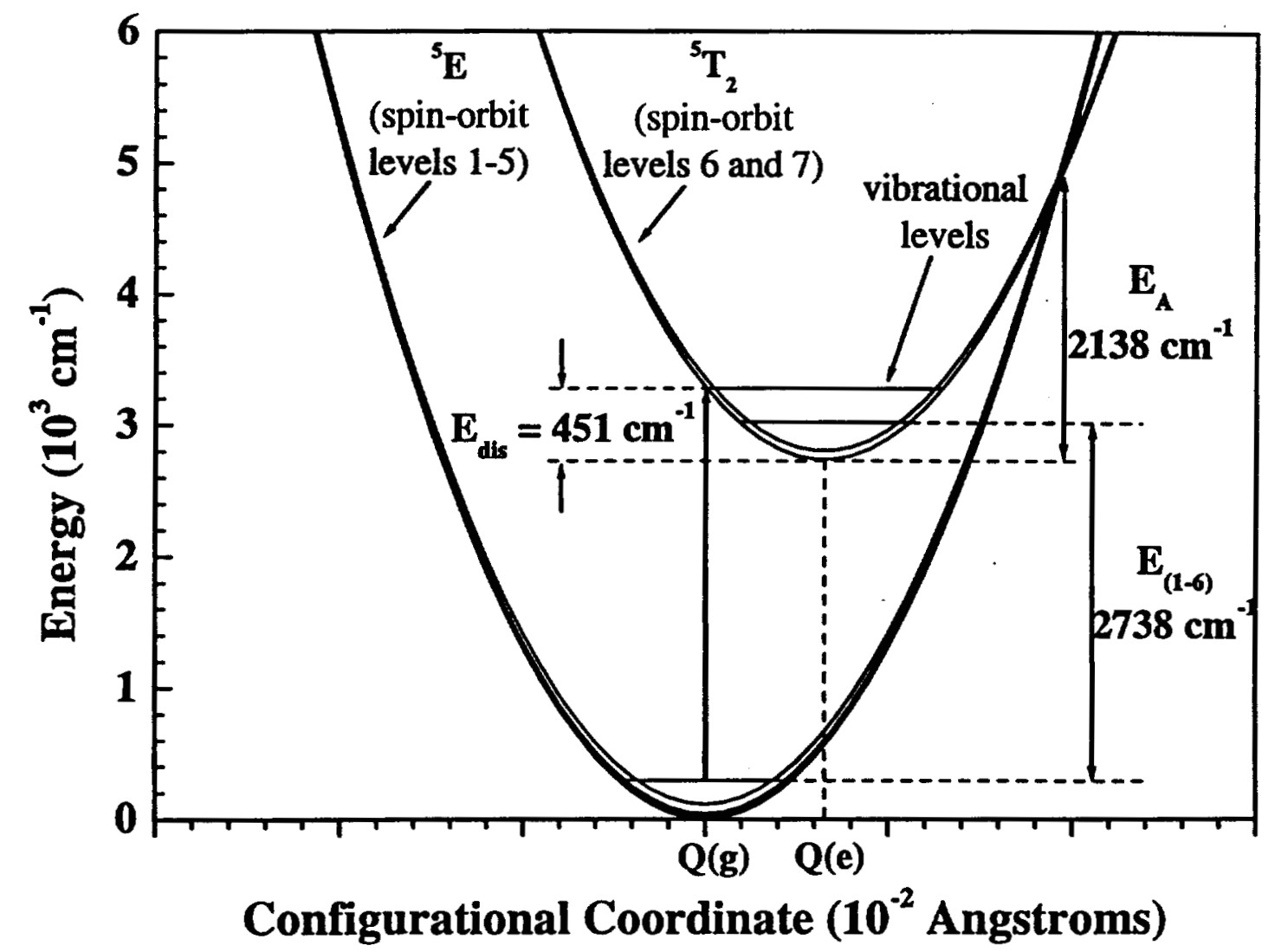

Figure 6.22. Configurational coordinate diagram scaled to values determined in this study for the ${ }^{5} \mathrm{~T}_{2}$ and ${ }^{5} \mathrm{E}$ states of $\mathrm{Fe}^{2+}: \mathrm{ZnSe}$. The parabolas represent potential energy surfaces for spin-orbit levels 1 through 7 shown in Fig. 6.2.

disproved by this work. The diagram in Fig. 6.22 illustrates the horizontal displacement between the ${ }^{5} \mathrm{E}$ and ${ }^{5} \mathrm{~T}_{2}$ states due to the linear interaction between the $\mathrm{Fe}^{2+}$ ions and the vibrating $\mathrm{ZnSe}$ lattice. 


\subsubsection{Energy level diagram}

The splittings in Fig. 6.2 are labeled with energy values in Fig. 6.23 based on the results from this spectroscopic study. The values for $4 \mathrm{Dq}$ and $6 \mathrm{Dq}$ were calculated from the value of $\Delta$ in Table 6.6 where $\Delta=10 \mathrm{Dq}$ [27]. The relative separations between spinorbit levels 1 through 5 and the separations between levels 6 through 11 are to scale. The separations and individual widths of the vibrational bands $\hbar \omega_{\text {peak A }}$ and $\hbar \omega_{\text {peak B }}$ are to scale. The ratio of the $4 \mathrm{Dq}$ and $6 \mathrm{Dq}$ separations are also to scale. However, the separations between levels 1 and 6 specified by $\mathrm{E}_{(1-6)}$ etc. and the magnitudes of the 4Dq and $6 \mathrm{Dq}$ spacings are not to scale.

The values for $E_{(8-6)}$ through $E_{(11-6)}$ were calculated using Eqns. (6.11) - (6.14) and $\Delta$ and $\lambda$ from Table 6.6. The calculated values have uncertainties of $\pm 50 \%$. The vibrational levels shown as thick bands (gray) on the far right of Fig. 6.23 represent the range of values determined for the phonon energies from the absorption spectrum. They should be regarded as the vibrational energies of phonons associated with level 6 and possibly level 7 due to the close proximity of levels 6 and 7 (i.e. $68 \mathrm{~cm}^{-1}$ ). The relation between the vibrational bands and levels 8 and 9 should be regarded as only coincidental since we do not know the positions of levels 8 and 9 accurately (i.e. an uncertainty of $\pm 50 \%$ ).

In deriving this energy level diagram we have neglected important effects like the static Jahn-Teller $[20,21]$ and the Ham effect $[26,32,44]$ which may have significant impact on the position of levels. Our main goal was to derive under simple assumptions a clear energy level scheme for $\mathrm{Fe}^{2+}: \mathrm{ZnSe}$ that is consistent with the low temperature spectra in order to gain an insight into the reasons for the behavior of the spectroscopic 


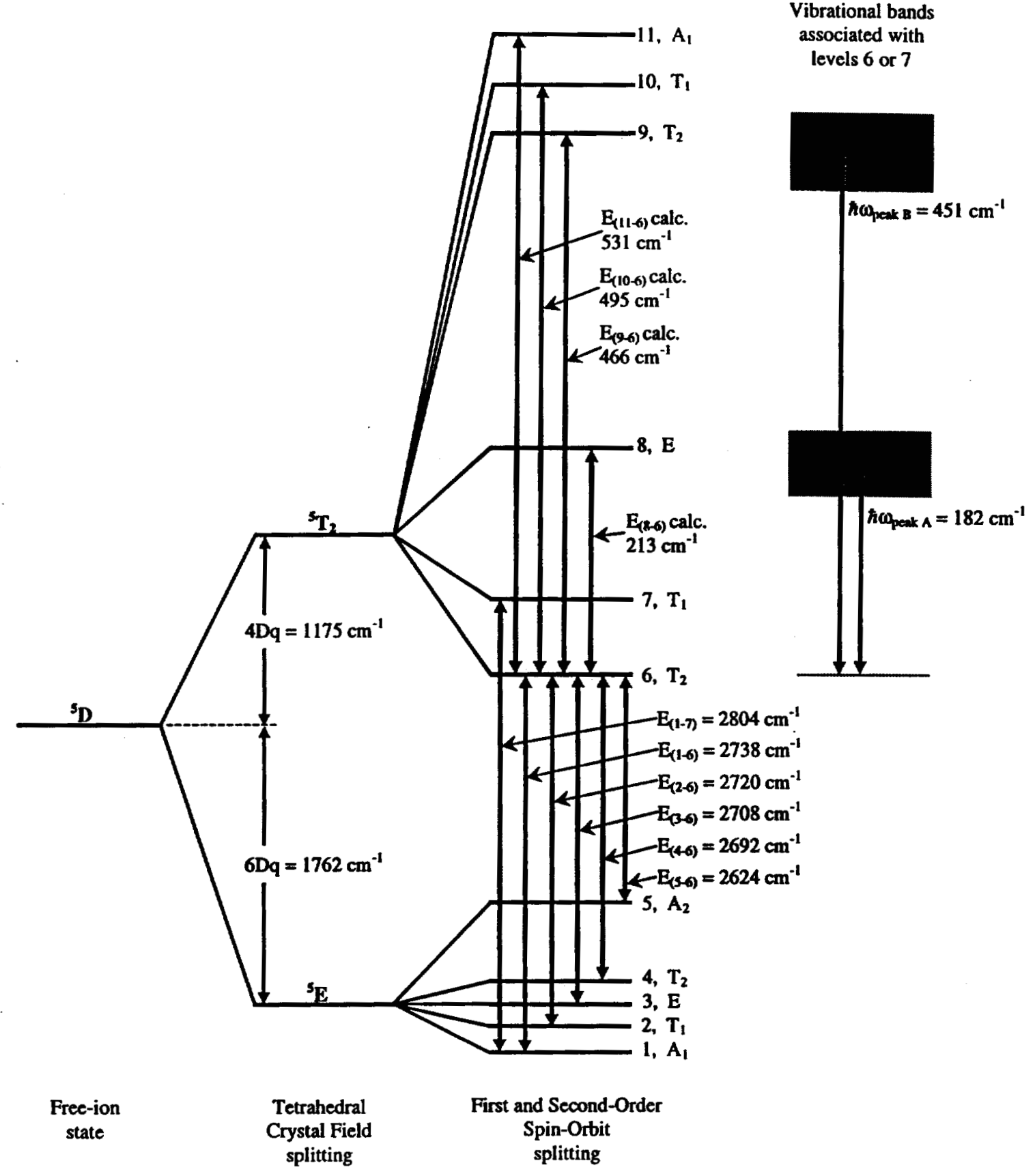

Figure 6.23. Magnitudes for the splittings of the ${ }^{5} \mathrm{D}$ free-ion state as found for $\mathrm{Fe}^{2+}: \mathrm{ZnSe}$. $E_{(8-6)}$ through $E_{(11-6)}$ were calculated using Eqns. (6.11)-(6.14). The gray bands represent the range of vibrational energies determined from the absorption spectrum.

properties with changing temperature. This simple diagram will also be useful in understanding the lasing properties of $\mathrm{Fe}^{2+}: \mathrm{ZnSe}$ at different temperatures. 


\subsection{Summary}

In order to evaluate the utility of $\mathrm{Fe}^{2+}: \mathrm{ZnSe}$ as a $3-5 \mu \mathrm{m}$ laser material a spectroscopic study in this wavelength range was conducted. The material was found to absorb between $2650 \mathrm{~nm}$ and $3800 \mathrm{~nm}$ which implied that an Er:YAG laser emitting at $2698 \mathrm{~nm}$ would be a suitable pump source for the emission, lifetime, and possible subsequent laser experiments. Upon excitation at $2698 \mathrm{~nm}$, the material had a strong emission band between $3900 \mathrm{~nm}$ and $4600 \mathrm{~nm}$, which suggests a potential laser tuning range over $700 \mathrm{~nm}$. The luminescence lifetime was found to be very dependent on temperature with values that ranged from $49 \mu$ s at $14 \mathrm{~K}$ to $5 \mu s$ at $220 \mathrm{~K}$. Fe ${ }^{2+}: \mathrm{ZnSe}$ did not luminesce for temperatures greater than approximately $225 \mathrm{~K}$ which suggests cryogenic temperatures will be necessary for laser operation. A configurational coordinate diagram and a detailed energy level diagram were constructed for the ${ }^{5} \mathrm{E}$ and ${ }^{5} \mathrm{~T}_{2}$ states in $\mathrm{Fe}^{2+}: \mathrm{ZnSe}$ based on the spectroscopic results presented here. The encouraging spectroscopic properties for $\mathrm{Fe}^{2+}: \mathrm{ZnSe}$ determined here and the availability of an Er:YAG pump laser warranted further evaluation of $\mathrm{Fe}^{2+}: \mathrm{ZnSe}$ as a potential 3 - 5 $\mu \mathrm{m}$ laser material as will be discussed in the next chapter. 


\section{References for Chapter 6}

[1] R. M. Measures, Laser Remote Sensing: Fundamentals and Applications, (John Wiley and Sons, New York, 1984)

[2] T. P. Grayson, et al., Proc. IEEE NAECON 2, 678 (1996)

[3] Handbook of Military Infrared Technology, W. L. Wolfe (ed.), (Naval Research Laboratory, U.S. Government Printing Office, 1965)

[4] H. A. Weakliem, J. Chem. Phys., 36, 2117 (1962)

[5] H. Uchiike, Electrolumin. Proc. Fourth Int. Workshop, S. Shionoya and H. Kobayashi (eds.), 238 (Springer-Verlag, Berlin, 1989)

[6] M. Yamaguchi and T. Shigematsu, Jpn. J. Appl. Phys. 17, 335 (1978)

[7] R. Pappalardo and R. E. Dietz, Phys. Rev. 123, 1188 (1961)

[8] J. T. Vallin, G. A. Slack, S. Roberts, and A. E. Hughes, Phys. Rev. B 2, 4313 (1970)

[9] H. Nelkowski and G. Grebe, J. Lumin. 1-2, 88 (1970)

[10] W. Busse, H.-E. Gumlich, E. Neumann, and D. Theiss, J. Lumin. 3, 351 (1971)

[11] P. Koidl, O. F. Schirmer, and U. Kaufmann, Phys. Rev. B 8, 4926 (1973)

[12] G. Grebe and H.-J. Schulz, Z. Nat. Forsch. A 29A, 1805 (1974)

[13] A. P. Radlinski, J. Phys. C, Solid-State Phys. 12, 4477 (1979)

[14] R. Renz and H.-J. Schulz, J. Lumin. 24-25, 221 (1981)

[15] G. Goetz and H.-J. Schulz, J. Lumin. 40-41, 415 (1988)

[16] G. Roussos, H.-J. Schulz, and M. Thiede, J. Lumin. 31-32, 406 (1984)

[17] D. J. Robbins, P. J. Dean, J. L. Glasper, and S. G. Bishop, Solid State Commun. 36, 61 (1980)

[18] G. Goetz, H. Zimmermann, and H.-J. Schulz, Z. Phys. B 91, 429 (1993)

[19] G. Roussos and H.-J. Schulz, J. Lumin. 31-32, 427 (1984)

[20] G. A. Slack, F. S. Ham, and R. M. Chrenko, Phys. Rev. 152, 376 (1966)

[21] G. A. Slack and B. M. O'Meara, Phys. Rev. 163, 335 (1967)

[22] J. H. Haanstra, II-VI Semiconducting Compounds 1967 International Conference,

D. G. Thomas (ed.), 207 (W. A. Benjamin, Inc., New York, 1967)

[23] P. B. Klein, J. E. Fumeaux, and R. L. Henry, Appl. Phys. Lett. 42, 638 (1983)

[24] L. D. DeLoach, R. H. Page, G. D. Wilke, S. A. Payne, and W. F. Krupke, IEEE J. Quan. Elec. 32, 885 (1996)

[25] R. H. Page, K. I. Schaffers, L. D. DeLoach, G. D. Wilke, F. D. Patel, J. B. Tassano, Jr., S. A. Payne, W. F. Krupke, K.-T. Chen, and A. Burger, IEEE J. Quan. Elec. 33, 609 (1997)

[26] R. C. Powell, Physics of Solid-State Laser Materials (Springer-Verlag, New York, 1998)

[27] F. A. Cotton, Chemical Applications of Group Theory, $3^{\text {rd }}$ Edition (Wiley, New York, 1971)

[28] S. Sugano, Y. Tanabe, and H. Kamimura, Multiplets of Transition-Metal Ions in Crystals, (Academic Press, New York, 1970)

[29] J. M. Baranowski, J. W. Allen, and G. L. Pearson, Phys. Rev. 160, 627 (1967)

[30] B. Henderson and G. F. Imbusch, Optical Spectroscopy of Inorganic Solids, (Clarendon Press, Oxford, 1989) 
[31] T. M. Dunn, D. S. McClure, and R. G. Pearson, Some Aspects of Crystal Field Theory, (Harper and Row, Publishers, New York, 1965)

[32] W. Low and M. Weger, Phys. Rev. 118, 1119 (1960)

[33] J. A. Caird, et al., IEEE J. Quan. Elec. 24, 1077 (1988)

[34] L. J. Andrews, A. Lempicki, B. C. McCollum, C. J. Giunta, R. H. Bartram, and J. F. Dolan, Phys. Rev. B 34, 2735 (1986)

[35] A. M. Stoneham, Theory of Defects in Solids, (Oxford University Press, London, 1985)

[36] S. A. Payne, L. L. Chase, and G. D. Wilke, J. Lumin. 44, 167 (1989)

[37] P. W. Atkins, Quanta A Handbook of Concepts, (Oxford University Press, Oxford, 1994)

[38] H. E. Ruda, Widegap II-VI Compounds for Opto-electronic Applications, (Chapman and Hall, London, 1992)

[39] C. L. Rablau, J.-O. Ndap, X. Ma, A. Burger, and N. C. Giles, J. Electron. Mater. 28, 678 (1999)

[40] S. A. Payne, L. L. Chase, H. W. Newkirk, L. K. Smith, and W. F. Krupke, IEEE J. Quan. Elec. 24, 2243 (1988)

[41] B. Hennion, F. Moussa, G. Pepy, and K. Kunc, Phys. Letters 36A, 376 (1971)

[42] C. Kittel, Introduction to Solid State Physics, (John Wiley and Sons, Inc., New York, 1996)

[43] C. J. Ballhausen, Introduction to Ligand Field Theory, (McGraw-Hill Book Company, Inc., New York, 1962)

[44] L. Podlowski, R. Heitz, P. Thurian, A. Hoffmann, and I. Broser, J. Lumin. 58, 252 (1994) 


\section{Chapter 7}

\section{0 - 4.5 $\mu \mathrm{m}$ laser performance of $\mathrm{Fe}^{2+}: \mathrm{ZnSe}$ below $180 \mathrm{~K}$}

First published as: J. J. Adams, C. Bibeau, R. H. Page, D. M. Krol, L. H. Furu, and S. A. Payne, "4.0 - $4.5 \mu \mathrm{m}$ lasing of Fe:ZnSe below $180 \mathrm{~K}$, a new mid-infrared laser material," Optics Letters, 24, 1720 (1999)

\subsection{Introduction}

The spectroscopic properties of $\mathrm{Fe}^{2+}: \mathrm{ZnSe}$ were studied in Chapter 6 in order to evaluate its potential as a $3-5 \mu \mathrm{m}$ laser material. Measured values of $\sim 10^{-18} \mathrm{~cm}^{2}$ for the absorption and emission cross-sections, luminescence lifetimes on the order of $50 \mu \mathrm{s}$, and the availability of an Er:YAG excitation laser encouraged us to pursue lasing experiments with $\mathrm{Fe}^{2+}: \mathrm{ZnSe}$. The Bridgman-grown $\mathrm{Fe}^{2+}: \mathrm{ZnSe}$ material (sample \#12163) had the strongest proclivity to luminesce and also exhibited the longest luminescence lifetimes and so the laser studies focused solely on the Bridgman-grown samples.

In the next section (section 7.2), we briefly review the spectroscopic properties of $\mathrm{Fe}^{2+}: \mathrm{ZnSe}$ most relevant to the lasing experiments. The Er:YAG laser used for the spectroscopic experiments had a maximum output energy of approximately $150 \mu \mathrm{J} / \mathrm{pulse}$. In section 7.3 , we estimate the pump energy needed to reach laser threshold in a $20 \mathrm{~cm}$ confocal cavity containing $\mathrm{Fe}^{2+}: \mathrm{ZnSe}$ as the gain media. Section 7.4 describes our experimental laser test setup and the results from the lasing experiments with $\mathrm{Fe}^{2+}: \mathrm{ZnSe}$. A summary of the results is given in section 7.5.

\subsection{Review of $\mathrm{Fe}^{2+}: \mathrm{ZnSe}$ optical spectroscopy}

As was discussed in Chapter $6, \mathrm{Fe}^{2+}: \mathrm{ZnSe}$ does not luminesce for temperatures above approximately $225 \mathrm{~K}$ because of thermally-activated nonradiative coupling to the 
vibrational modes of the $\mathrm{ZnSe}$ lattice. Therefore this mandates cryogenic laser $(<225 \mathrm{~K})$ operation. We review in Fig. 7.1 the low temperature absorption and emission spectra measured for $\mathrm{Fe}^{2+}: \mathrm{ZnSe}$ in the last chapter. The Er:YAG pump laser's wavelength $\left(\lambda_{\text {pump }}\right)$ is labeled in the figure. We see from the overlap of the absorption and emission spectra, that at this temperature we would expect $\mathrm{Fe}^{2+}: \mathrm{ZnSe}$ to have a lasing wavelength $\cong 3.9$ $\mu \mathrm{m}$. This wavelength is expected because in the range of emission wavelengths without

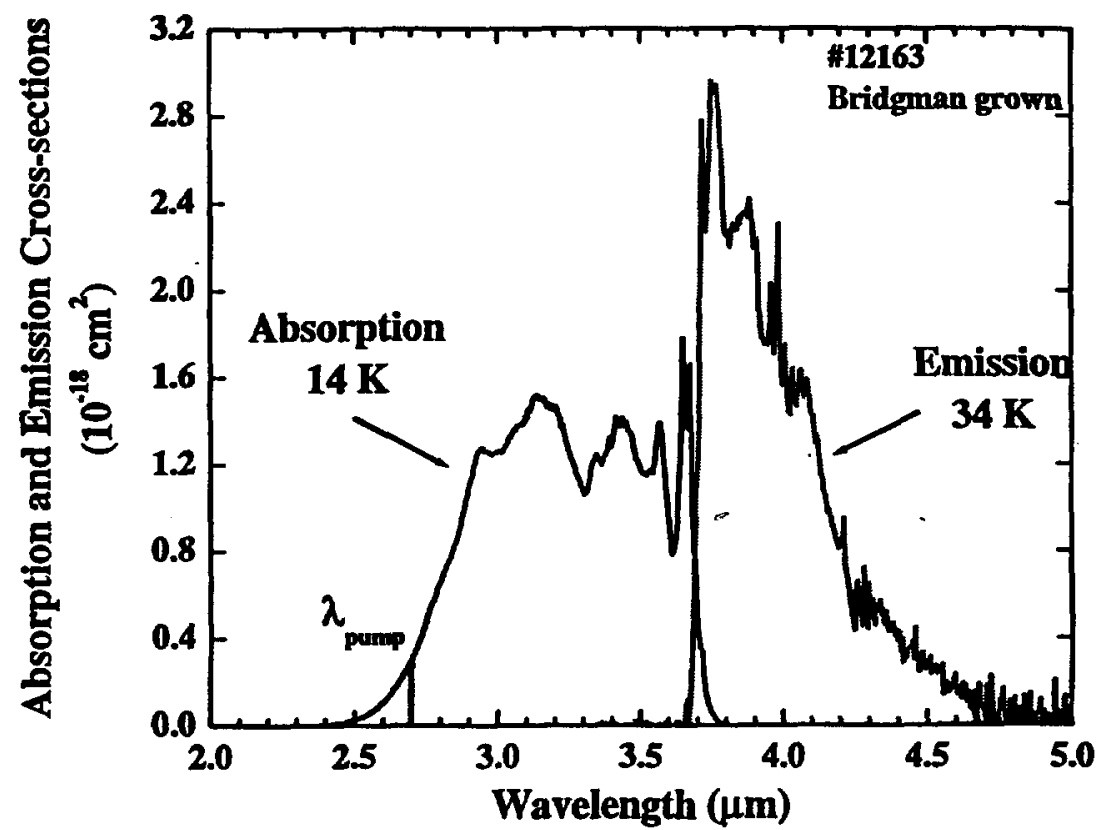

Figure 7.1. Unpolarized low temperature absorption and emission cross-sections for $\mathrm{Fe}^{2+}: \mathrm{ZnSe}$ sample \#12163. The Er:YAG pump laser wavelength $\left(\lambda_{\text {pump }}\right)$ of $2.698 \mu \mathrm{m}$ is labeled.

absorption loss this is where the emission cross-section is largest. The wavelength of the Er:YAG pump laser $(2.698 \mu \mathrm{m})$ and the range of emission wavelengths for $\mathrm{Fe}^{2+}: \mathrm{ZnSe}$ were used to specify the spectral response of the dielectric coatings on the mirrors used to construct the laser test cavity.

$\mathrm{Fe}^{2+}: \mathrm{ZnSe}$ was found to have a luminescence lifetime that varied with temperature as 
shown in Fig. 7.2. The increase in lifetime from approximately $14 \mathrm{~K}$ to $110 \mathrm{~K}$ was found to be primarily due to radiation trapping (section 6.3.4) and the decrease in lifetime above

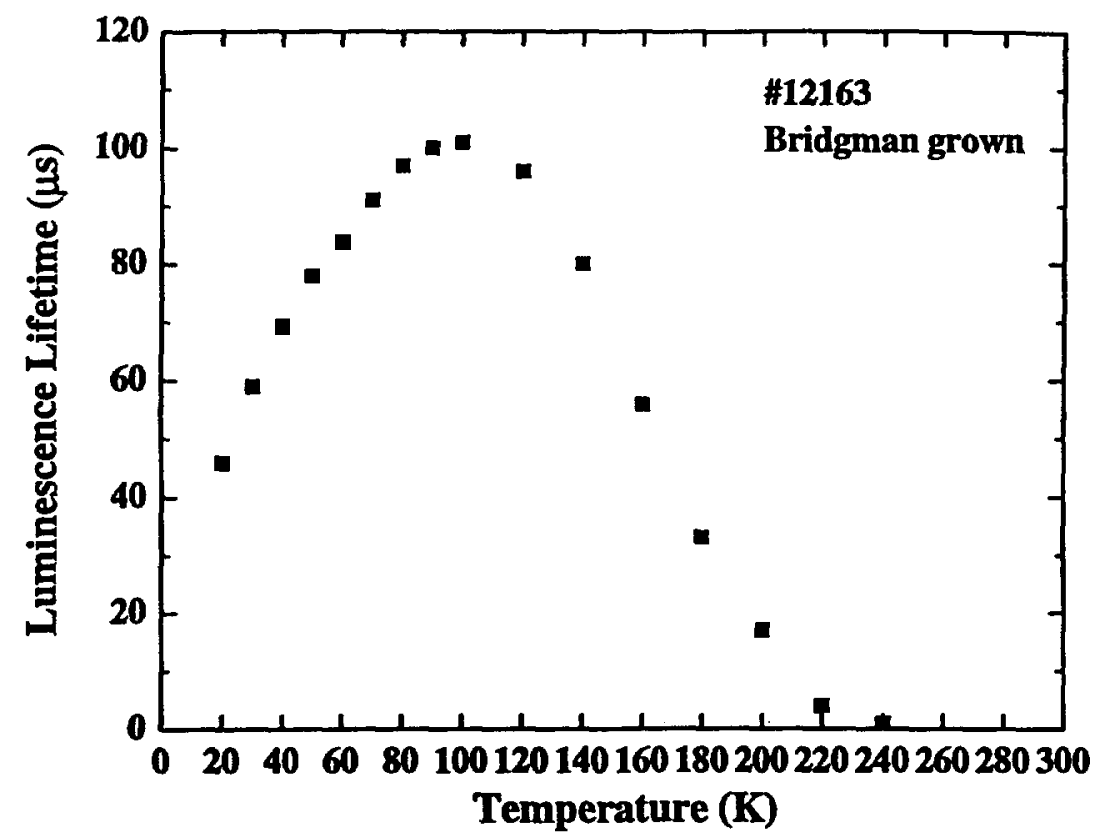

Figure 7.2. Luminescence lifetime as a function of temperature for $\mathrm{Fe}^{2+}: \mathrm{ZnSe}$ sample \#12163.

$110 \mathrm{~K}$ is due to increasing thermally-activated nonradiative decay as described by Eqn. (6.15). The magnitudes ( 10's $\mu s)$ of the lifetimes for $\mathrm{Fe}^{2+}: \mathrm{ZnSe}$ do not place any particularly demanding requirements on the pump laser's pulselengths.

\subsection{Laser threshold calculation}

Before procuring special cavity mirrors (i.e. highly transmissive at $2.698 \mu \mathrm{m}$ and highly reflective at $\sim 4 \mu \mathrm{m}$ ), we calculated the energy that the Er:YAG laser would need to deliver in order for the $\mathrm{Fe}^{2+}: \mathrm{ZnSe}$ laser to reach threshold. The estimate was based on the measured spectroscopic parameters for $\mathrm{Fe}^{2+}: \mathrm{ZnSe}$ at $14 \mathrm{~K}$ and a longitudinallypumped $20 \mathrm{~cm}$ confocal cavity arrangement. Eqn. (7.1) [1] can be used to calculate the 


$$
\mathrm{E}_{\mathrm{th}}=\frac{\pi h v_{\mathrm{p}}\left(\mathrm{w}_{\mathrm{p}}^{2}+\mathrm{w}_{\mathrm{s}}^{2}\right)(\mathrm{L}+\mathrm{T})}{4 \sigma_{\mathrm{em}} \tau_{\text {lum }}(1-\exp (-\alpha \ell))} \frac{\tau_{\mathrm{p}}}{\left(1-\exp \left(-\tau_{\mathrm{p}} / \tau_{\text {lum }}\right)\right)}
$$

incident pump pulse energy necessary to reach laser threshold, where $v_{p}$ is the frequency of the pump light, $w_{p}$ and $w_{s}$ are the pump and laser spot radii respectively, $L$ is the passive cavity loss, $T$ is the cavity output coupling, $\sigma_{\mathrm{em}}$ is the $34 \mathrm{~K}$ emission cross-section at the laser wavelength (assumed to be $3.9 \mu \mathrm{m}$ ), $\tau_{\text {lum }}$ is the luminescence lifetime at $14 \mathrm{~K}$ (49 $\mu \mathrm{s}), \alpha$ is the $14 \mathrm{~K}$ absorption coefficient at the pump wavelength $\left(\alpha=N \sigma_{a b s}=(3.4 x\right.$ $10^{19} \mathrm{Fe}$ atoms $\left.\left./ \mathrm{cm}^{3}\right)\left(0.296 \times 10^{-18} \mathrm{~cm}^{2}\right)=10.064 \mathrm{~cm}^{-1}\right), \ell$ is the crystal thickness $(0.198$ $\mathrm{cm})$, and $\tau_{\mathrm{p}}$ is the pump pulse duration. This formalism neglects ground state absorption $[1,2]$.

The pump beam was focused into the crystal using $\mathrm{a} \mathrm{CaF}_{2}$ lens with a focal length of $+20 \mathrm{~cm}$. The pump spot size $\left(w_{p}\right)$ at the crystal was measured with a razor scan technique in the vertical and horizontal directions. The pump spot size used for the threshold calculation was $210 \pm 50 \mu \mathrm{m}$. This value is the average of the measured horizontal and vertical pump spot sizes at the crystal. The laser mode was assumed to be a Gaussian $\mathrm{TEM}_{00}$ mode and its spot size within the $\mathrm{Fe}^{2+}: \mathrm{ZnSe}$ crystal was calculated using $\mathrm{w}_{\mathrm{s}}=\mathrm{w}_{\mathrm{o}}$ $\equiv(L \lambda / 2 \pi)^{1 / 2}=352 \mu \mathrm{m}[3]$ where $L=$ cavity length $=20 \mathrm{~cm}$. The inaccuracies in knowing the pump and laser spot sizes at the crystal could introduce errors into the threshold calculation as high as $30 \%$.

We assumed a passive loss (L) of 0.10 and a cavity output coupling $(T)$ of 0.005 for the threshold calculation. The $14 \mathrm{~K} \mathrm{Fe}^{2+}: \mathrm{ZnSe}$ emission cross-section at $3.9 \mu \mathrm{m}$ was determined from Fig. 7.1 to be $2.41 \times 10^{-18} \mathrm{~cm}^{2}$. The Er:YAG laser emits at $2.698 \mu \mathrm{m}$ in 
$48 \mu$ s long pulses at a repetition rate of $100 \mathrm{~Hz}$. At $14 \mathrm{~K}$, we calculate a threshold pulse energy of $\sim 8 \mu$ J. The Er:YAG laser could supply an order of magnitude more energy (i.e. $\sim 150 \mu \mathrm{J})$ than this estimated threshold value, therefore threshold was expected to be reached. In light of the compelling information determined for $\mathrm{Fe}^{2+}: \mathrm{ZnSe}$, we decided to have dielectric mirrors fabricated that would be used to construct a laser test cavity around the cryogenically-cooled $\mathrm{Fe}^{2+}: \mathrm{ZnSe}$ crystal.

\section{$7.4 \mathrm{Fe}^{2+}: \mathrm{ZnSe}$ laser experiments}

\subsubsection{Lasing demonstration}

A diagram of the experimental laser test setup is shown in Fig. 7.3. A cryogenic chamber (Air Products Displex Model 202) containing the cooled $\mathrm{Fe}^{2+}: \mathrm{ZnSe}$ crystal was placed between two identical $20 \mathrm{~cm}$ spherical concave mirrors forming a $20 \mathrm{~cm}$ confocal cavity. The faces of the $\mathrm{Fe}^{2+}: \mathrm{ZnSe}$ crystal perpendicular to the cavity axis were uncoated, but polished to within 22 seconds of parallel. The sample was longitudinally-pumped using the Er:YAG laser which had an output energy of approximately $150 \mu \mathrm{J} /$ pulse. A $3.3 \mu \mathrm{m}$ long-pass filter was attached to the InSb detector to block any pump light that passed through the cavity. At $19 \mathrm{~K}$, lasing was observed with a threshold pump energy of 1.2 $\mu \mathrm{J}$. We note that the observed threshold energy is lower than the calculated value. If the measured threshold value is used to back-calculate the passive loss, we determine a passive loss of 0.012 . This value is not extreme considering that scatter loss could be the

primary passive loss mechanism if Fresnel losses are canceled by near-parallel polish of the crystal surfaces. 


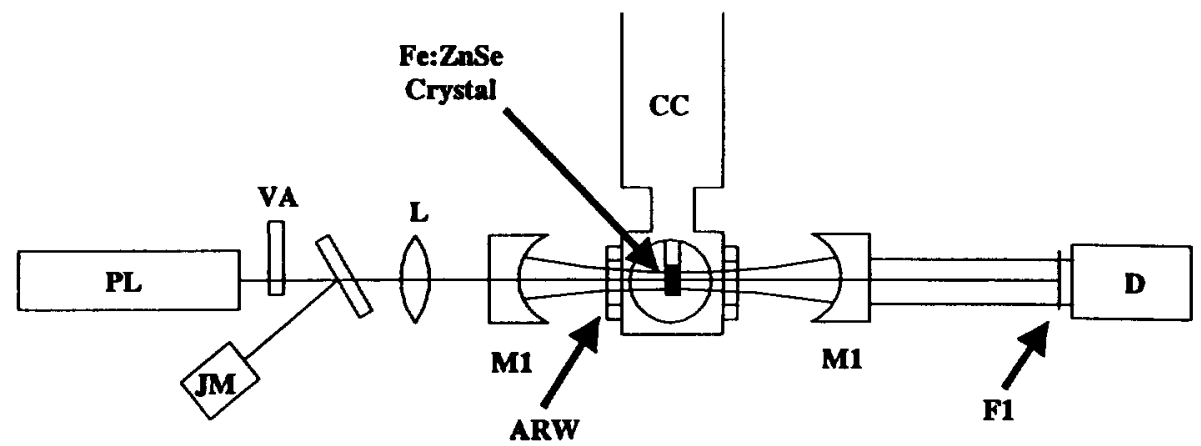

Figure 7.3. Schematic of the $\mathrm{Fe}^{2+}: \mathrm{ZnSe}$ laser system: PL, Er:YAG pump laser operating at $2.698 \mu \mathrm{m}$; VA, variable attenuator wheel; JM, Molectron J3-09 joulemeter; $\mathrm{L},+20 \mathrm{~cm}$ $\mathrm{CaF}_{2}$ lens; $\mathrm{M1}, 20 \mathrm{~cm}$ radius of curvature $\mathrm{CaF}_{2}$ mirrors with $\mathrm{T}=82 \%$ at $2.698 \mu \mathrm{m}$ and $\mathrm{T}$ $=0.3 \%$ at $3.98 \mu \mathrm{m} ; \mathrm{ARW}$, anti-reflection coated $\mathrm{CaF}_{2}$ window (at both the pump and the laser wavelengths); $\mathrm{CC}, 20-300 \mathrm{~K}$ cryogenic vacuum chamber; $\mathrm{F} 1,3.3 \mu \mathrm{m}$ long-wave pass filter; D, E. G. \& G. Judson liquid nitrogen cooled InSb detector.

\subsubsection{Temperature tuning}

The wavelength and output energy of the $\mathrm{Fe}^{2+}: \mathrm{ZnSe}$ laser was observed to change with temperature. The lasing wavelength was measured at several temperatures using a $1 / 3$ meter, 150 groove/mm CVI Instruments Digikrom 240 computer controlled monochromator, a Labview data acquisition program, and a liquid nitrogen cooled InSb detector. This equipment was placed in the position of the detector on the right side of Fig. 7.3. The output energy was measured at several temperatures using the same basic experimental setup as shown in Fig. 7.3 except that the InSb detector was replaced by a Molectron J3-09 joulemeter. The incident energy from the Er:YAG pump laser was kept constant and the output energy from the $\mathrm{Fe}^{2+}: \mathrm{ZnSe}$ laser was measured while the temperature of the crystal was varied in discrete steps.

Fig. 7.4 shows a comparison between the spontaneous emission spectra and the laser emission for $\mathrm{Fe}^{2+}: \mathrm{ZnSe}$ at three approximately equivalent temperatures. The $\mathrm{Fe}^{2+}: \mathrm{ZnSe}$ laser's emission at $18 \mathrm{~K}$ was centered at $3.98 \mu \mathrm{m}$ with a bandwidth of $0.007 \mu \mathrm{m}$. The 
increased laser linewidth at $130 \mathrm{~K}$ shown in Fig. 7.4 indicates that the $\mathrm{Fe}^{2+}: \mathrm{ZnSe}$ laser was operating in multiple longitudinal modes [3]. The shift in output of the $\mathrm{Fe}^{2+}: \mathrm{ZnSe}$ laser to longer wavelengths as the temperature of the crystal increased is an effect resulting from higher lying spin-orbit and vibrational states of ${ }^{5} E$ being thermally populated at higher temperatures causing increased losses at the shorter wavelengths. Fig. 7.5 shows the output wavelength for the $\mathrm{Fe}^{2+}: \mathrm{ZnSe}$ laser at 5 different temperatures, which varied from $3.98 \mu \mathrm{m}$ at $18 \mathrm{~K}$ to $4.54 \mu \mathrm{m}$ at $180 \mathrm{~K}$. The $\mathrm{Fe}^{2+}: \mathrm{ZnSe}$ laser is thus temperature tunable from at least 3.98 to $4.54 \mu \mathrm{m}$.

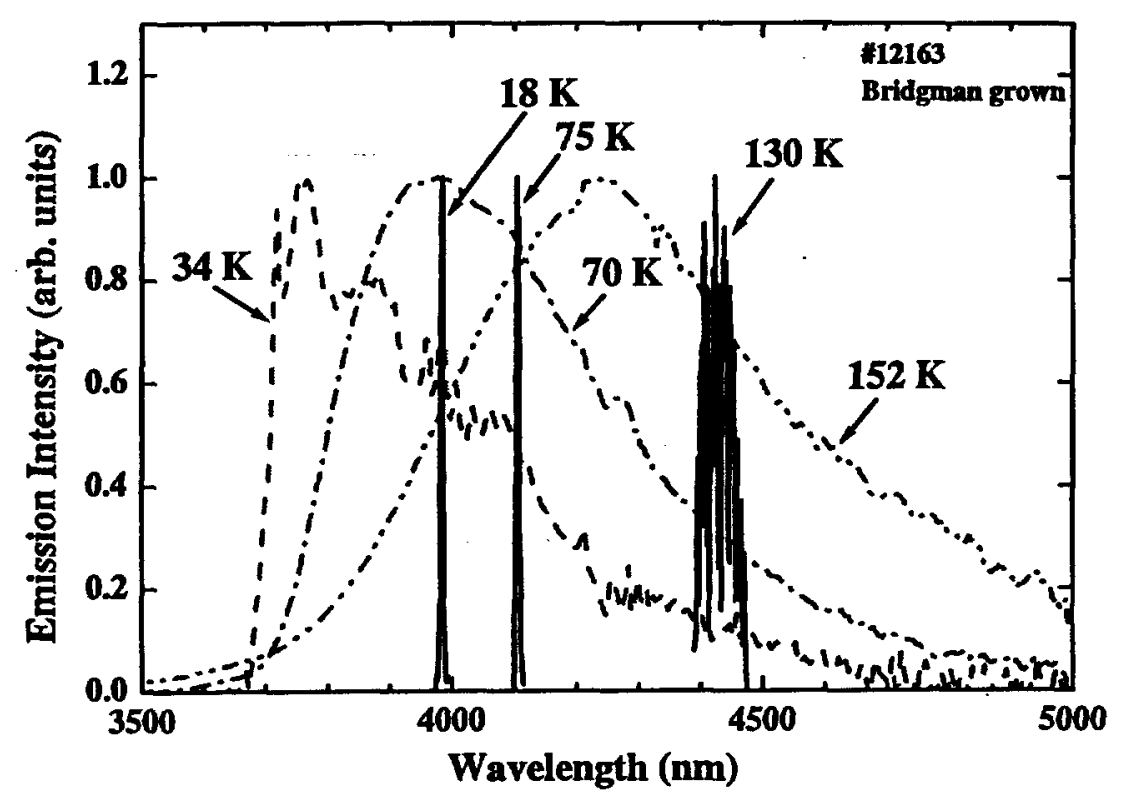

Figure 7.4. Spontaneous emission spectra at $34 \mathrm{~K}, 70 \mathrm{~K}$, and $152 \mathrm{~K}$ and laser emission at $18 \mathrm{~K}, 75 \mathrm{~K}$, and $130 \mathrm{~K}$ for $\mathrm{Fe}^{2+}: \mathrm{ZnSe}$.

The $\mathrm{Fe}^{2+}: \mathrm{ZnSe}$ laser produced its highest output energy at' $150 \mathrm{~K}: 48 \mu$ s long pulses with a maximum energy per pulse of $7.8 \mu \mathrm{J}$ for an incident pump energy of $108.2 \mu \mathrm{J}$. The low observed level of output energy between approximately $40 \mathrm{~K}$ and $90 \mathrm{~K}$ as shown in Fig. 7.5 is believed to be due to the strong atmospheric $\mathrm{CO}_{2}$ absorption near $4.23 \mu \mathrm{m}$ 


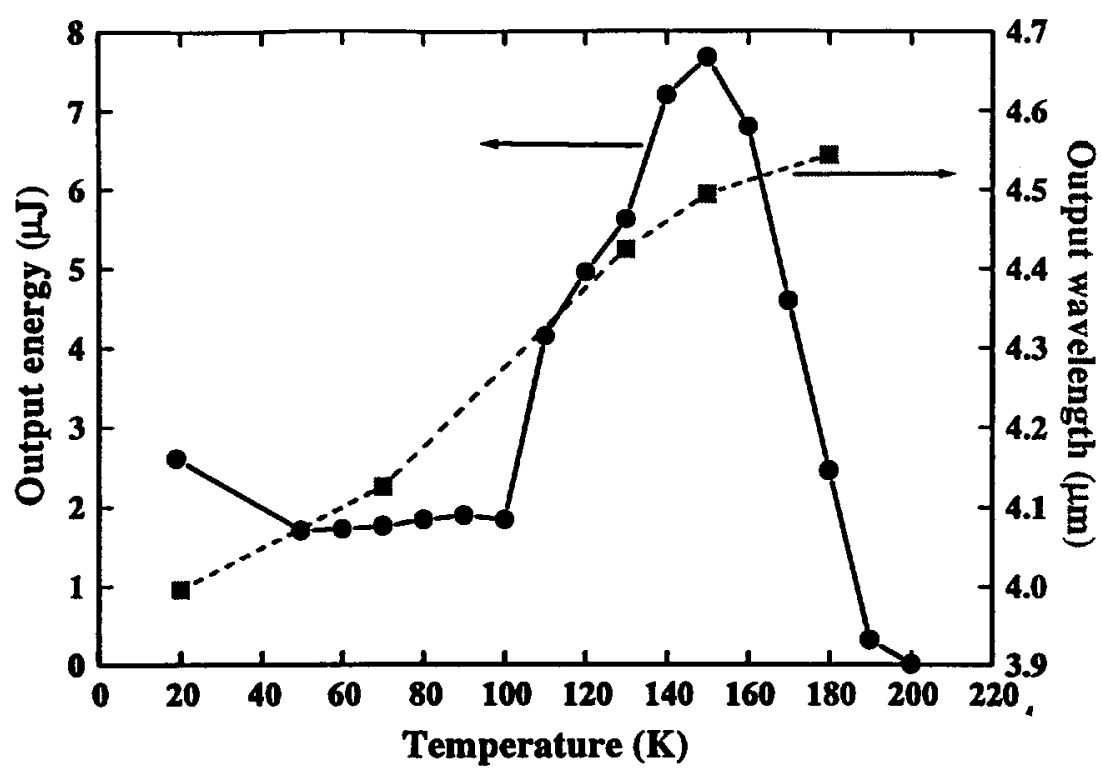

Figure 7.5. Variation of the $\mathrm{Fe}^{2+}: \mathrm{ZnSe}$ laser wavelength and output energy with crystal temperature. The lines through the data are only to aid the eye.

which would significantly attenuate the $\mathrm{Fe}^{2+}: \mathrm{ZnSe}$ laser's output. No effort was made to purge the $\mathrm{CO}_{2}$ in the space of the laser and detector. Another factor contributing to the relatively low output energy below $100 \mathrm{~K}$ is the relatively low absorption at the pump wavelength. Therefore the increase in output energy of the $\mathrm{Fe}^{2+}: \mathrm{ZnSe}$ laser from approximately $100 \mathrm{~K}$ to $150 \mathrm{~K}$ can be attributed in part to the increasing absorption at the pump wavelength as shown in Fig. 6.6.

The variation in output energy and wavelength with temperature measured here for the $\mathrm{Fe}^{2+}: \mathrm{ZnSe}$ laser is very similar to the temperature behavior that has been observed for the Alexandrite laser [4]. The $\mathrm{Fe}^{2+}: \mathrm{ZnSe}$ laser is therefore a vibronic laser [5]. Vibronic lasers have lasing transitions that emit both photons and phonons. The amount of energy emitted as phonons can be varied continuously, for instance through a change in temperature, and therefore the energy (wavelength) of the photons also varies 
continuously. The result is broad wavelength tunability in the laser output (i.e. as shown in Fig. 7.5).

\subsubsection{Laser slope efficiencies}

Laser slope efficiencies were determined at several temperatures for an output coupling of $0.6 \%$ as shown in Fig. 7.6. The slope efficiency data was collected with the

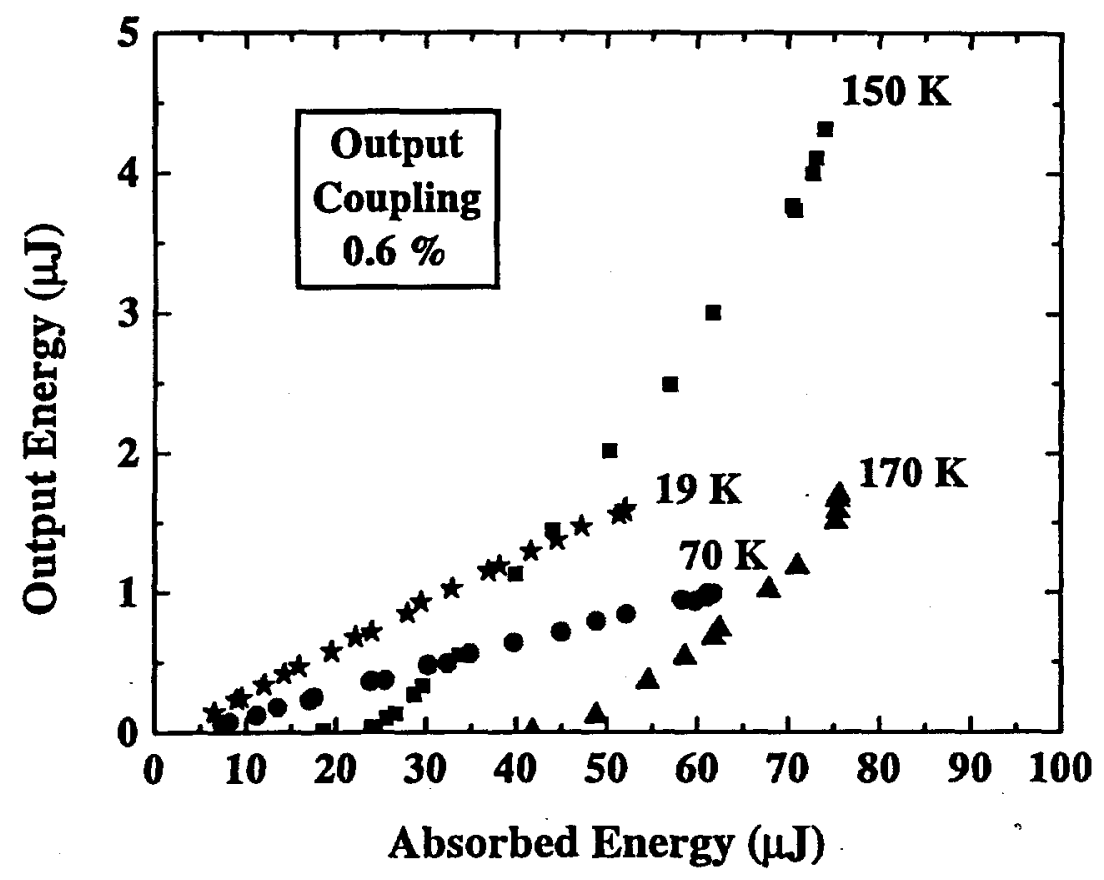

Figure 7.6. $\mathrm{Fe}^{2+}: \mathrm{ZnSe}$ laser output energy vs. absorbed $2.698 \mu \mathrm{m}$ pump energy at $19 \mathrm{~K}$,

$70 \mathrm{~K}, 150 \mathrm{~K}$, and $170 \mathrm{~K}$ for $100 \mathrm{~Hz}$ operation.

same basic experimental setup as in Fig. 7.3, except that the InSb detector was replaced by a Molectron J3-09 joulemeter. The output energy of the $\mathrm{Fe}^{2+}: \mathrm{ZnSe}$ laser was measured as a function of the incident pump energy at each temperature. The incident pump energy was varied using the attenuator wheel shown in Fig. 7.3. For a $0.6 \%$ output coupling, Table 7.1 lists the slope efficiencies and laser thresholds determined at four different temperatures for the $\mathrm{Fe}^{2+}: \mathrm{ZnSe}$ laser. The slope efficiencies were determined 
Table 7.1. Measured slope efficiencies and laser thresholds for the $\mathrm{Fe}^{2+}: \mathrm{ZnSe}$ laser at different crystal temperatures.

\begin{tabular}{|c|c|c|}
\hline Temperature & Slope Efficiency & Laser Threshold \\
\hline $19 \mathrm{~K}$ & $3.2 \%$ & $1.2 \mu \mathrm{J}$ \\
\hline $70 \mathrm{~K}$ & $1.7 \%$ & $3.0 \mu \mathrm{J}$ \\
\hline $150 \mathrm{~K}$ & $8.2 \%$ & $24.5 \mu \mathrm{J}$ \\
\hline $170 \mathrm{~K}$ & $5.2 \%$ & $46.3 \mu \mathrm{J}$ \\
\hline
\end{tabular}

from the slope of a linear fit to the data in Fig. 7.6 and the threshold values were the $x$ intercepts of the fitted lines. The slope efficiency is proportional to the inverse of the round-trip cavity loss [6]. Therefore the decrease in slope efficiency from $19 \mathrm{~K}$ to $70 \mathrm{~K}$ is in part due to the $\mathrm{CO}_{2}$ absorption loss mentioned earlier and the decrease from $150 \mathrm{~K}$ to $170 \mathrm{~K}$ is in part due to the increased thermally-activated nonradiative losses. Even though rather low slope efficiencies were obtained, improvement can be made by optimizing the cavity output coupling and employing an anti-reflection coating on the crystal. The increase in the threshold values with temperature is partially due to the increase in overlap between the absorption and emission wavelengths causing the lasing wavelength to increase which decreases the emission cross-section (Fig. 7.1). Another contributing factor to the increase in threshold is that the luminescence lifetime over these temperatures is primarily decreasing which by Eqn. (7.1) increases the threshold.

\subsection{Summary}

In summary, lasing action has been demonstrated for the first time in $\mathrm{Fe}^{2+}: \mathrm{ZnSe}$, thereby verifying $\mathrm{Fe}^{2+}$ as a tunable mid-infrared laser ion. The $\mathrm{Fe}^{2+}: \mathrm{ZnSe}$ laser produced a maximum output energy of $7.8 \mu \mathrm{J} /$ pulse at $150 \mathrm{~K}$ for a pump pulse energy of $108 \mu \mathrm{J}$. The $\mathrm{Fe}^{2+}: \mathrm{ZnSe}$ laser tuned with temperature from $3.98 \mu \mathrm{m}$ to $4.54 \mu \mathrm{m}$. The $\mathrm{Fe}^{2+}: \mathrm{ZnSe}$ laser had low laser thresholds $(-1-50 \mu \mathrm{J})$ and slope efficiencies between $2 \%$ and $10 \%$. 
Improvement in the slope efficiencies can be afforded by optimizing the cavity output coupling and improving the crystal quality. 


\section{References for Chapter 7}

[1] P. F. Moulton, IEEE J. Quan. Elec. 21, 1582 (1985)

[2] W. P. Risk, J. Opt. Soc. Am. B 5, 1412 (1988)

[3] A. E. Siegman, Lasers (University Science Books, Sausalito, 1986)

[4] S. Guch, Conference on Lasers and Electro-Optics 1983 (Baltimore, MD) paper ThR3

[5] W. Koechner, Solid-State Laser Engineering, $4^{\text {th }}$ Edition (Springer-Verlag, Berlin, 1996)

[6] F. D. Patel, Ph.D. dissertation (University of California, Davis, CA, 2000) 


\section{Chapter 8}

\section{Summary}

This dissertation presented the results from an exploratory study to identify and characterize new crystalline materials for frequency conversion of near-infrared wavelengths and as gain media for tunable mid-infrared solid-state lasers.

In the first four chapters, the $\mathrm{COB}$ family of nonlinear crystals (i.e. $\mathrm{LaCa}_{4} \mathrm{O}\left(\mathrm{BO}_{3}\right)_{3}$ $(\mathrm{LaCOB}), \quad \mathrm{GdCa}_{4} \mathrm{O}\left(\mathrm{BO}_{3}\right)_{3} \quad(\mathrm{Gd}, \mathrm{COB}), \quad \mathrm{YCa} \mathrm{O}_{4}\left(\mathrm{BO}_{3}\right)_{3} \quad(\mathrm{YCOB}), \quad$ and $\left.\mathrm{Gd}_{0.275} \mathrm{Y}_{0.725} \mathrm{Ca} 4 \mathrm{O}\left(\mathrm{BO}_{3}\right)_{3}(\mathrm{Gd}, \mathrm{YCOB})\right)$ were investigated for potential use in frequency conversion of $1 \mu \mathrm{m}$ lasers. In Chapter 2, the fundamental concepts of nonlinear optics used in Chapters $3-5$ were discussed. Chapter 3 reported the results from the study of second harmonic generation (SHG) in the COB family of crystals. Type I second harmonic generation (SHG) at $1064 \mathrm{~nm}$ was characterized in GdCOB, YCOB, Gd,YCOB, and the newest member to this family LaCOB. A $\mathrm{d}_{\text {eff }}$ of $0.52 \pm 0.05 \mathrm{pm} / \mathrm{V}$ and an angular sensitivity of $1224 \pm 184(\mathrm{~cm}-\mathrm{rad})^{-1}$ were determined for LaCOB. GdCOB and YCOB were found to have effective nonlinear coupling coefficients $\left(\mathrm{d}_{\mathrm{eff}}\right)$ of $0.78 \pm 0.06$ and $1.12 \pm 0.07 \mathrm{pm} / \mathrm{V}$, respectively and angular sensitivities of $2704 \pm 156$ and $4548 \pm 277$ (cm-rad $^{-1}$, respectively. The $d_{12}$ and $d_{32}$ coefficients of the nonlinear optical tensor for $\mathrm{LaCOB}, \mathrm{GdCOB}$, and $\mathrm{YCOB}$ were determined to be identical within the experimental uncertainty between the three crystals and have absolute values of 10.26 $\pm 0.04 \mid$ and $11.69 \pm 0.171 \mathrm{pm} / \mathrm{V}$, respectively. The effective coupling coefficient for type II non-critically phasematched (NCPM) doubling at $1064 \mathrm{~nm}$ in $\mathrm{Gd}_{0.275} \mathrm{Y}_{0.725} \mathrm{Ca}_{4} \mathrm{O}\left(\mathrm{BO}_{3}\right)_{3}$ was measured to be $0.37 \pm 0.04 \mathrm{pm} / \mathrm{V}$.

From phasematching angle measurements for type I SHG at 1064 and $1047 \mathrm{~nm}$ in 
LaCOB, it is predicted that LaCOB has a type I NCPM SHG wavelength of $1042 \pm 1.5$ nm. LaCOB, GdCOB and YCOB were observed to have very similar thermal sensitivities for type I SHG at $1064 \mathrm{~nm}$. The thermal sensitivity of type I SHG at 1064 $\mathrm{nm}$ in $\mathrm{LaCOB}$ is estimated to be less than $0.10\left(\mathrm{~cm}-{ }^{\circ} \mathrm{C}\right)^{-1}$ (i.e. 10 times less than type II $\left.\mathrm{LiB}_{3} \mathrm{O}_{5}\right)$. Due to its low angular and thermal sensitivities for doubling in the $1 \mu \mathrm{m}$ range, LaCOB has strong potential for use as an external and intracavity frequency doubler. The work in Chapter 3 was aimed at finding new crystals with type I NCPM SHG wavelengths near $1064 \mathrm{~nm}$. LaCOB was predicted to have a type I NCPM SHG wavelength of $1042 \pm 1.5 \mathrm{~nm}$. It was also determined that the $d_{32}$ coupling coefficient has a relatively large magnitude of $1.69 \mathrm{pm} / \mathrm{V}$ and so the $\mathrm{COB}$ crystals were further characterized in Chapters 4 and 5 for two applications based on this coefficient.

In Chapter 4, the COB crystals were evaluated for use in a non-critically phasematched optical parametric oscillator (OPO) in the $2 \mu \mathrm{m}$ range. Experiments were conducted to measure the type II NCPM $\underline{\mathrm{SHG}}$ wavelength down the $\mathrm{x}$ dielectric axis of the COB crystals in order to determine the degenerate oscillation wavelength for a type II NCPM OPO. To accomplish this, a $\mathrm{LiNbO}_{3} \mathrm{OPO}$ was constructed as a tunable pump source and type II phasematched SHG was observed down the $\mathrm{x}$ dielectric axis in YCOB, GdCOB, and LaCOB as a function of the pump wavelength. Efforts to determine the exact (within $1 \mathrm{~nm}$ ) non-critically phasematched SHG wavelength down the $\mathrm{x}$ dielectric axis for the COB crystals were unsuccessful. However, our best results indicate that the non-critically phasematched wavelength was near $1725 \pm 50 \mathrm{~nm}$. Several experimental improvements are suggested to determine this wavelength to within $1 \mathrm{~nm}$.

Chapter 5 presented the measurements of the effective linear electro-optic coefficients 
( $\left.r_{\text {eff }}\right)$ along dielectric directions in $\mathrm{YCOB}$ for two longitudinal and four transverse configurations. For YCOB, a maximum $r_{\text {eff }}$ of $10.7 \pm 1.0 \mathrm{pm} / \mathrm{V}$ for the longitudinal configurations and a maximum $\mathrm{r}_{\mathrm{eff}}$ of $10.81 \pm 1.46 \mathrm{pm} / \mathrm{V}$ for the transverse configurations was determined. Therefore in a longitudinal configuration, the corresponding half-wave voltage at $1064 \mathrm{~nm}$ would be $99 \mathrm{kV}$. This may be put in perspective by comparison to the commonly utilized electro-optic crystal $\mathrm{BaB}_{2} \mathrm{O}_{4}$ (BBO). $\mathrm{BBO}$ has a half-wave voltage at $1064 \mathrm{~nm}$ of $48 \mathrm{kV}$. Alternatively, in a transverse configuration, a YCOB crystal with a 5:1 aspect ratio would have a half-wave voltage at $1064 \mathrm{~nm}$ of $19.6 \mathrm{kV}$.

One potential electro-optic use that was identified for YCOB was a multiple-plate longitudinal Pockels cell. The advantage of using multiple plates is that the required half-wave voltage could be much less than $99 \mathrm{kV}$. A potential transverse electro-optic application for YCOB would be to use a pair of identical YCOB crystals each with a 5:1 (L:d) aspect ratio separated by a $90^{\circ}$ rotator. This thermally compensated device would have a half-wave voltage $<10 \mathrm{kV}$. Therefore, the most probable electro-optic application for YCOB is in a large aperture $1 \mu \mathrm{m}$ Q-switch.

- The second part of this dissertation (Chapters 6 and 7) was the evaluation of $\mathrm{Fe}^{2+}: \mathrm{ZnSe}$ as a widely tunable mid-infrared laser material. In Chapter 6, a spectroscopic study of $\mathrm{Fe}^{2+}: \mathrm{ZnSe}$ was conducted in the $3-5 \mu \mathrm{m}$ range. $\mathrm{Fe}^{2+}: \mathrm{ZnSe}$ was found to have a broad absorption band from 2650 to $3900 \mathrm{~nm}$ and a broad emission band from 3900 to $4600 \mathrm{~nm}$ at a temperature of $34 \mathrm{~K}$. Because of the strong coupling to the vibrational modes of the $\mathrm{ZnSe}$ lattice, $\mathrm{Fe}^{2+}: \mathrm{ZnSe}$ was not observed to luminesce at temperatures above $225 \mathrm{~K}$. However, because of the coupling to the lattice, broad vibrational sidebands appear in the spectra which allow $\mathrm{Fe}^{2+}: \mathrm{ZnSe}$ to have wide tunability (i.e. a 
vibronic laser material). Absorption cross-sections of $\sim 1.2 \times 10^{-18} \mathrm{~cm}^{2}$ and emission cross-sections of $\sim 1.6 \times 10^{-18} \mathrm{~cm}^{2}$ for temperatures below $220 \mathrm{~K}$ were experimentally determined. The luminescence lifetime was found to be very dependent on temperature and ranged from 5 to $110 \mu$ s for temperatures below $220 \mathrm{~K}$. After accounting for radiation trapping, we estimated the radiative lifetime for $\mathrm{Fe}^{2+}: \mathrm{ZnSe}$ to be $49 \mu \mathrm{s}$ for an $\mathrm{Fe}$ concentration of $\sim 10^{19}$ atoms $/ \mathrm{cm}^{3}$. A configurational coordinate diagram and a detailed energy level diagram were constructed for the ${ }^{5} \mathrm{E}$ and ${ }^{5} \mathrm{~T}_{2}$ states in $\mathrm{Fe}^{2+}: \mathrm{ZnSe}$ based on the experimentally determined spectroscopic results.

In Chapter 7, lasing action was demonstrated for the first time in $\mathrm{Fe}^{2+}: \mathrm{ZnSe}$ for temperatures below $200 \mathrm{~K}$. The $\mathrm{Fe}^{2+}: \mathrm{ZnSe}$ laser produced $48 \mu$ s long pulses, and at 150 $\mathrm{K}$, had a maximum output energy of $7.8 \mu \mathrm{J} /$ pulse for an absorbed pump energy of $108 \mu \mathrm{J}$. The $\mathrm{Fe}^{2+}: \mathrm{ZnSe}$ laser was tuned with temperature from $3.98 \mu \mathrm{m}(20 \mathrm{~K})$ to $4.54 \mu \mathrm{m}(180$ K). The $\mathrm{Fe}^{2+}: \mathrm{ZnSe}$ laser had very low laser thresholds between $1-50 \mu \mathrm{J}$ and slope efficiencies between 2 and $10 \%$.

In conclusion: the $\mathrm{COB}$ family of nonlinear crystals have unique potential for frequency conversion of large aperture high average power lasers, LaCOB has especially strong potential for external and intracavity frequency doubling of Nd:YLF and $\mathrm{Yb}^{3+}: \mathrm{Sr}_{5}\left(\mathrm{PO}_{4}\right)_{3} \mathrm{~F}(\mathrm{Yb}: \mathrm{S}-\mathrm{FAP})$ lasers, $\mathrm{YCOB}$ is a promising material for use in large aperture, high-average power $1 \mu \mathrm{m}$ Q-switches, and $\mathrm{Fe}^{2+}: \mathrm{ZnSe}$ can be utilized as a widely tunable $3-5 \mu \mathrm{m}$ laser material with a tuning range of over $500 \mathrm{~nm}$.

It is the hope of the author that the study of the COB crystals presented in this dissertation provides motivation for future work that will continue the exploration of new applications for these crystals. One such application for the COB crystals may be as an 
integrated self-doubled, Q-switched gain medium. It is also the hope of the author that the study of $\mathrm{Fe}^{2+}: \mathrm{ZnSe}$ presented will inspire other researchers to pursue other transition metal-host combinations that will offer new emission wavelengths combined with wide tunability. 